\title{
GRAY CODES, UNIVERSAL CYCLES AND CONFIGURATION ORDERINGS FOR BLOCK DESIGNS
}

\author{
Megan Dewar \\ A thesis submitted to the Faculty of Graduate Studies \\ Carleton University \\ in partial fulfillment of the requirements \\ for the degree of Doctor of Philosophy in the \\ Ottawa-Carleton Institute for Graduate Studies and Research \\ in Mathematics and Statistics
}

(c)2007 Megan Dewar 


$\begin{array}{ll}\begin{array}{l}\text { Library and } \\ \text { Archives Canada }\end{array} & \begin{array}{l}\text { Bibliothèque et } \\ \text { Archives Canada }\end{array} \\ \begin{array}{l}\text { Published Heritage } \\ \text { Branch }\end{array} & \begin{array}{l}\text { Direction du } \\ \text { Patrimoine de l'édition }\end{array} \\ \begin{array}{l}\text { 395 Wellington Street } \\ \text { Ottawa ON K1A ON4 }\end{array} & \begin{array}{l}\text { 395, rue Wellington } \\ \text { Ottawa ON K1A ON4 } \\ \text { Canada }\end{array}\end{array}$

Your file Votre référence ISBN: 978-0-494-33488-1 Our file Notre référence ISBN: 978-0-494-33488-1

NOTICE:

The author has granted a nonexclusive license allowing Library and Archives Canada to reproduce, publish, archive, preserve, conserve, communicate to the public by telecommunication or on the Internet, loan, distribute and sell theses worldwide, for commercial or noncommercial purposes, in microform, paper, electronic and/or any other formats.

The author retains copyright ownership and moral rights in this thesis. Neither the thesis nor substantial extracts from it may be printed or otherwise reproduced without the author's permission.
AVIS:

L'auteur a accordé une licence non exclusive permettant à la Bibliothèque et Archives Canada de reproduire, publier, archiver, sauvegarder, conserver, transmettre au public par télécommunication ou par l'Internet, prêter, distribuer et vendre des thèses partout dans le monde, à des fins commerciales ou autres, sur support microforme, papier, électronique et/ou autres formats.

L'auteur conserve la propriété du droit d'auteur et des droits moraux qui protège cette thèse. $\mathrm{Ni}$ la thèse ni des extraits substantiels de celle-ci ne doivent être imprimés ou autrement reproduits sans son autorisation.
In compliance with the Canadian

Privacy Act some supporting forms may have been removed from this thesis.

While these forms may be included in the document page count, their removal does not represent any loss of content from the thesis.
Conformément à la loi canadienne sur la protection de la vie privée, quelques formulaires secondaires ont été enlevés de cette thèse.

Bien que ces formulaires aient inclus dans la pagination, il n'y aura aucun contenu manquant.

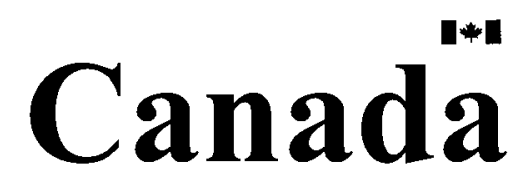




\section{Abstract}

The problem of determining minimal change orderings for binary vectors, $k$-subsets of $n$-sets, permutations and other combinatorial objects has been thoroughly explored in the context of combinatorial Gray codes. Further work has been done within the restricted framework of Universal cycles. In this dissertation, we consider minimal change orderings for the blocks of designs. We define Gray codes and Universal cycles for designs and present existence results. We also extend the definition of configuration ordering and establish the existence of new configuration orderings for the blocks of designs.

A $\kappa$-intersecting Gray code for a $(v, k, \lambda)$-BIBD, $\mathcal{S}$, is a listing of the blocks of $\mathcal{S}$ such that consecutive blocks intersect in exactly $\kappa$ points. We prove that there exists a twofold triple system of order $v$ for each $v \equiv 1,3,4,7(\bmod 12)$, with sporadic exceptions, admitting a 2-intersecting Gray cycle. A Universal cycle (Ucycle) is a more restricted form of minimal change ordering. We define Ucycles for designs and look at two realizations of this definition. In one realization, blocks of the design 
in question are represented based on their cardinality or block size $(k)$, while in the other realization, representation is based on the strength of the design (which is two for $(v, k, \lambda)$-BIBDs). With rank equal to block size, we look at twofold triple systems, proving both existence and non-existence results for Ucycles of rank three. With rank equal to strength, we prove that Ucycles of rank two exist for all cyclic BIBDs with a sufficient number of non-regular base blocks. Results are proved by construction.

The concept of ordering the blocks of a design is not limited to Gray codes and Ucycles. A $(p, \ell)$-configuration is a set system with $p$ elements and $\ell$ blocks in which every element is contained in at least one block. Given a configuration $C$ having $\ell$ blocks, a $C$-ordering for a design is a listing of the blocks of the design such that every $\ell$ consecutive blocks form a configuration isomorphic to $C$. We generalize this concept to sets of configurations. We establish results in both standard $\left(A_{1}, B_{1}\right.$ and $\left.B_{2}\right)$ and generalized $\left(\left\{B_{4}, B_{5}\right\}\right)$ configuration ordering for triple systems and BIBDs. 


\section{Acknowledgments}

The following people and organizations have supported me throughout my $\mathrm{PhD}$ studies.

My supervisor, Brett Stevens, has been encouraging and inspiring. His interest and enthusiasm for this research has spurred me on and under his guidance I have learned a great deal.

My partner, Chris North, has been supportive throughout this degree (and the previous two). His calm approach to life and general positive outlook have been a necessary counterpoint to my panic and stress. Despite his claims that he has no idea what I'm talking about, I have clarified and solidified many ideas as a result of our discussions on various topics related to this research.

My Mum, Dad and sister have always been encouraging and supportive.

My friend Karen Meagher read many chapters of this thesis. More importantly, she kept me sane through several analysis courses and entertained me through several others. 
My friend John Proos read this entire thesis more diligently than I could have hoped. His corrections and suggestions have greatly improved this document.

John Poland recognized my interest in teaching mathematics and furthered that interest through discussion and example. His genuine concern for people around him made the difficult first year back at school a little less painful.

The National Science and Engineering Research Council provided generous financial support.

Megan Dewar

Ottawa, 2007 


\section{Contents}

Abstract

Acknowledgments iv

1 Introduction $\quad 1$

2 Background $\quad 15$

2.1 Designs . . . . . . . . . . . . . . . . . 16

2.1.1 Balanced Incomplete Block Designs . . . . . . . . . . . . 16

2.1 .2 Automorphisms of BIBDs . . . . . . . . . . 20

2.1.3 Pairwise Balanced Designs . . . . . . . . . . . 27

2.1.4 Block Intersection Graphs . . . . . . . . . . . . 28

2.2 Configurations . . . . . . . . . . . . . . . . . . . . 29

2.3 Binary Gray Codes . . . . . . . . . . . . . . . . 33

2.3.1 Standard Binary Reflected Gray Cycles . . . . . . . . . . . 34

vi 
2.3.2 Balanced and Monotone Gray Codes . . . . . . . . . 37

2.4 Combinatorial Gray Codes . . . . . . . . . . . . . . 40

$2.4 .1 \quad k$-subsets of $n$-sets $\ldots \ldots \ldots 40$

2.4 .2 Permutations ..................... 45

2.5 De Bruijn Sequences $\ldots \ldots \ldots$. . . . . . . . . . . . 47

2.6 Universal Cycles . . . . . . . . . . . . . . . . . . . . . 52

2.6 .1 Permutations . . . . . . . . . . . . . 53

$2.6 .2 k$-subsets of $n$-sets $\ldots \ldots \ldots 64$

2.6 .3 Partitions of an $n$-set $\ldots \ldots \ldots 71$

$\begin{array}{lll}3 & \text { Ordering the blocks of designs } & 75\end{array}$

3.1 Gray Codes for Block Designs . . . . . . . . . . . . 76

3.2 Universal Cycles for Block Designs . . . . . . . . . . . . 78

3.3 Configuration Ordering . . . . . . . . . . . . . 84

3.3.1 Ordering the Blocks of Steiner Triple Systems for Application to Erasure-Correcting Codes . . . . . . . . . . . 86

3.3.2 Translated Results in Configuration Ordering . . . . . . . 95

3.3.3 Generalized Configuration Ordering . . . . . . . . . . . 99

4 Gray Codes and Universal Cycles for Designs 101

4.1 Gray Codes and Universal Cycles for TTSs . . . . . . . . . . . . 102

vii 
4.2 Gray Codes and Universal Cycles for BIBDs . . . . . . . . . . . 135

5 New Results in Configuration Ordering 218

5.1 Standard Configuration Ordering . . . . . . . . . . . . . 219

5.2 Generalized Configuration Ordering . . . . . . . . . . . . . 243

5.3 Configuration Ordering for BIBDs . . . . . . . . . . . . . 248

6 Conclusions and Future Work $\quad 264$

6.1 Approaches to Determining the Existence of Rank Two Universal Cy-

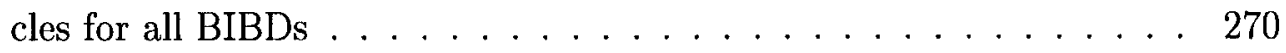

6.2 Conjectures and Questions . . . . . . . . . . . . . . 283

6.2 .1 Conjectures . . . . . . . . . . . . . . 283

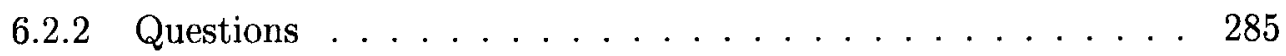

$\begin{array}{lr}\text { Bibliography } & 289\end{array}$

$\begin{array}{lr}\text { Index } & 297\end{array}$

viii 


\section{Chapter 1}

\section{Introduction}

The mathematical sciences particularly exhibit order, symmetry and limitation, and these are the greatest forms of the beautiful.

- Aristotle, Metaphysica

In this dissertation, we consider the problem of ordering the blocks of designs to meet specified criteria. The problems of ordering binary vectors, $k$-subsets of $n$ sets, permutations and other combinatorial objects have been thoroughly explored in the context of combinatorial Gray codes. Further work has been done within the restricted framework of Universal cycles. These existing definitions will motivate the criteria for ordering blocks. We give definitions of Gray codes and Universal cycles for designs and present existence results. We also extend the definition of configuration ordering and establish the existence of new configuration orderings for the blocks of 
designs.

The most common and well-studied ordering concept in combinatorics is the Gray code. Gray codes are well-studied from a theoretical standpoint and, in addition, have many practical applications. Gray codes are particularly efficient in testing situations where there is a cost associated to making adjustments to inputs between tests. The name "Gray code" is due to Frank Gray who, in 1953, used a binary reflected code in a pulse code communication system. A binary Gray code of order $n$ lists all $2^{n}$ strings of $n$-bits such that only one bit changes from one string to the next, in a simple and regular way. A binary Gray code is cyclic if the change rule also holds from the last $n$-bit string to the first $n$-bit string. In general terms, a combinatorial Gray code for a collection of combinatorial objects is a listing such that successive elements differ in a small, pre-specified way. As Gray codes have both practical applications and appealing properties, their existence, enumeration and generation has been wellstudied (see Savage's survey paper [47] for an extensive list of papers). While a Gray code for $k$-subsets of an $n$-set (with various minimal change properties) is a Gray code for the family of $\left(n, k,\left(\begin{array}{c}n-2 \\ k-2\end{array}\right)\right)$-BIBDs, and the existence of such codes is known $[5,7,11,23,45,46,59]$, Gray codes for designs have not been studied in general.

There are many ways to define minimal change. The minimality may refer to where the change occurs or may refer to how the change occurs. While these two concepts are not mutually exclusive, the standard binary reffected Gray code clearly 
falls into the first category; the change at each step is specified by the bit position that is to be changed. The ordered list of bit positions that change (known as a transition sequence) completely specifies the code. An example of the second type of change is a de Bruijn sequence. A de Bruijn sequence of order $n$ is a listing of all $n$-bit strings such that successive strings differ by a shift one position left (dropping the first element) followed by the introduction of a new last element. In other words, this form of change asks that consecutive elements of the de Bruijn sequence be successive states of a queue obtained by a pre-specified series of simultaneous pushes and pops. Here the change at each step is given by the value that is to be pushed onto the queue. This form of change means that successive elements of the sequence may differ radically in each bit position (that is, successive elements may have large Hamming distance), however, the numbers of zeros and ones appearing may not differ at all. This definition allows us to compress the list of all $n$-bit strings into a single cyclic list of $2^{n}$ bits.

Formally, a $k$-ary de Bruijn sequence of order $n$ is a circular sequence of length $k^{n}$ such that every $n$-tuple from the alphabet $\mathbb{Z}_{k}$ appears as a contiguous subsequence. First investigated by Flye-Sainte Marie in 1894 and later rediscovered by N. de Bruijn, de Bruijn sequences have long been known (Sanskrit memory wheels) and extensively used (fault testing, pseudo-random number generation, magicians' tricks). De Bruijn sequences of order $n$ on alphabets of size $k$ are known to exist for all $n \in \mathbb{N}$ and all 
$k \in \mathbb{N}$. A natural abstraction of this concept was introduced by Chung, Diaconis and Graham in their 1992 paper "Universal cycles for combinatorial structures" [12]. In this paper they propose representing combinatorial objects (other than the $n$-tuples on $\mathbb{Z}_{k}$ ) in a cyclic sequence such that each object appears exactly once. Formally, suppose $\mathcal{F}_{n}$ is a family of combinatorial objects of 'rank' $n$ and let $m=\left|\mathcal{F}_{n}\right|$. Rank refers to the size of the representative of each object. This term is loosely defined as each type of combinatorial object may be represented differently. For example, $k$-subsets of an $n$-set may represent themselves, while partitions of an $n$-set may be represented by vectors of length $n$ where the value in the $i^{\text {th }}$ position indicates the partition to which element $i$ belongs. Assume each $F \in \mathcal{F}_{n}$ is specified by some sequence $\left\langle x_{0}, x_{1}, \ldots, x_{n-1}\right\rangle$, where, for $0 \leq i \leq n-1, x_{i} \in A$ for some fixed alphabet $A$. The sequence $U=a_{0}, a_{1}, \ldots, a_{m-1}$ is a Universal cycle (Ucycle) for $\mathcal{F}_{n}$ if $\left\langle a_{i+1}, \ldots, a_{i+n}\right\rangle$, $0 \leq i<m$, runs through each element of $\mathcal{F}_{n}$ exactly once, where index addition is performed modulo $m$. The paper sparked a flurry of work which produced results on the existence of Ucycles for $k$-subsets of an $n$-set, permutations of an $n$-set and partitions of an $n$-set. The existence of Ucycles for $k$-permutations of an $n$-set, $2<$ $k<n$, is completely determined [39] as is the existence of Ucycles for partitions of an $n$-set [12]. The problem is not entirely solved for $n$-permutations of an $n$-set $[37,38]$ and existence of Ucycles for $k$-subsets of an $n$-set is far from complete. It is known that Ucycles for $k$-subsets of an $n$-set, where $k=3,4,6$, exist when $n$ is sufficiently 
large and coprime to $k$, and provided $\left(\begin{array}{c}n-1 \\ k-1\end{array}\right) \equiv 0(\bmod k)[35,38,39]$. Notice that a Ucycle for subsets exhibits both minimal change properties; consecutive elements differ by a shift and also differ by one element (since order of elements in a subset is irrelevant). We can think of Ucycles for subsets as a minimal change listing in which the number that has been "in the subsets" longest is changed. This is similar in flavour to the definition of a Beckett Gray code [22].

In this dissertation, we consider the question of whether minimal change orderings exist for block designs. We investigate both Gray codes and the more restricted form Ucycles. The natural questions to ask are: how do we define such sequences? Do these sequences exist for all designs? If so, how many are there and how do we construct them? In order to answer these questions, we first address an important issue that arises in the search for Ucycles for any combinatorial object. Unlike de Bruijn sequences, there are many ways to represent the combinatorial objects in question and how objects are represented affects whether Ucycles can be found. We define Ucycles for block designs and look at two realizations of this definition. In one realization, blocks of the design in question are represented based on their cardinality or block size, while in the other realization, representation is based on the strength of the design. First we look at twofold triple systems of order $v(\operatorname{TTS}(v) \mathrm{s})$, which exist for all $v \equiv 0,1(\bmod 3)$. We prove there exists a $\operatorname{TTS}(v)$ for each $v \equiv 1,4,7(\bmod 12)$, with sporadic exceptions, that admits a Ucycle of rank three. When $v \equiv 0,3,6,9$ 
(mod 12), we show that Ucycles of rank three for $\operatorname{TTS}(v)$ s cannot exist. We prove there exists a $\operatorname{TTS}(v)$ for each $v \equiv 3(\bmod 12)$ that admits a Gray cycle in which consecutive objects share two points. This will be called a 2-intersecting Gray cycle. Second, we prove that Ucycles of rank two exist for all cyclic BIBDs having a sufficient number of non-regular base blocks. We prove existence by construction, thereby answering the third question. Techniques often seen in Gray code construction will be applied to the construction of Ucycles for block designs, indicating that Ucycles for designs are a natural extension of this concept.

The concept of ordering the blocks of a design is not limited to Gray codes and Ucycles. In 2003, Cohen and Colbourn introduced the idea of a configuration ordering for the blocks of a design [15]. A $(p, \ell)$-configuration is a set system with $p$ elements and $\ell$ blocks in which every element is contained in at least one block. Let $C$ be a configuration having $\ell$ blocks. A configuration ordering, or $C$-ordering, for a design is a listing of the blocks of the design such that every $\ell$ consecutive blocks form a configuration isomorphic to $C$. If this property also holds when we treat the list as a cycle, then the ordering is called $C$-cyclic. Cohen and Colbourn asked, for various configurations $C$, when does there exist a Steiner triple system of order $v(\operatorname{STS}(v))$ for which the triples can be $C$-ordered? Of course, this question can be asked of all triple systems and block designs in general. 
Configurations are easiest to express visually. Figures 1 and 2 represent the configurations on blocks of size three that we discuss in this chapter. We employ the labeling of configurations given by Colbourn and Rosa in [19] and Griggs and Grannell in [26]. Note that specifying the number of blocks and the number of points may not uniquely determine a configuration. For example, $B_{3}$ and $B_{4}$ (Figure 2) are both $(7,3)$-configurations. In such cases, we will fully describe the configuration in question. As defined above, configurations need not have blocks of size three, however, there is no standard notation that describes these more general configurations. Given a configuration $C$ with blocks of size three, we will use the notation $C^{\prime}$ to refer to the generalized version of this configuration (describing the generalization in detail where necessary).

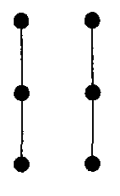

$\mathrm{A}_{1}$

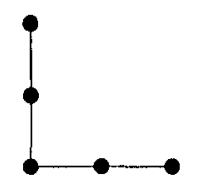

$\mathrm{A}_{2}$

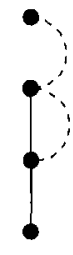

$\mathrm{A}_{3}$

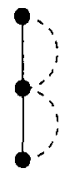

$\mathrm{A}_{4}$

Figure 1: Configurations on two lines (labels from [19] and [26])

While few people have looked directly at existence of configuration orderings, many known results can be expressed in this language. Simmons and Davis proved that all $(v, 2,1)$-BIBDs admit $(2 d, d)$-orderings if and only if $d \leq\lfloor(v-3) / 2\rfloor[49]$. Note that for any $m, k \in \mathbb{N}$, the $(m \cdot k, k)$-configuration consists of $k$ mutually disjoint 


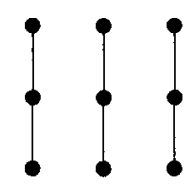

$\mathrm{B}_{1}$

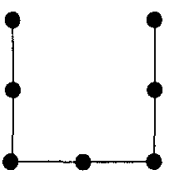

$\mathrm{B}_{4}$

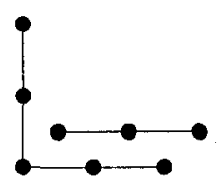

$\mathrm{B}_{2}$

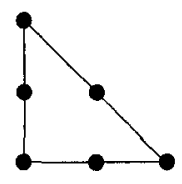

$\mathrm{B}_{5}$

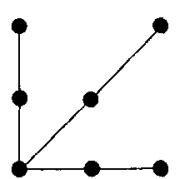

$\mathrm{B}_{3}$

Figure 2: Configurations on three lines with $\lambda=1$ (labels from [19] and [26])

blocks of size $m$. Horák and Rosa proved that every $(v, k, 1)$-BIBD admits an $A_{2}^{\prime}$-cyclic ordering (see Figure 1) by proving that the 1-block intersection graph of every $(v, k, 1)$ BIBD is Hamiltonian [33]. The $i$-block intersection graph of a design $S=(V, \mathcal{B})$ is the graph formed by taking blocks as vertices and connecting two vertices if the blocks they represent intersect in exactly $i$ points. Two groups of authors enhanced Horák and Rosa's result. Horák, Pike and Raines showed that all triple systems of order greater than or equal to twelve admit $A_{2}$-cyclic orderings [32], while the work of Alspach, Heinrich and Mohar implies that any $(v, K, 1)$-PBD (where $K$ represents a collection of integers, each less than $v$ ) with $\max (K) \leq 2 \cdot \min (K)$ admits a cyclic configuration ordering in which pairs of consecutive blocks intersect in exactly one point [2]. Hare proved that the 1-block intersection graph of every $(v, K, 1)$-PBD with $\min (K) \geq 3$ is edge-pancyclic; a result which implies such 1-block intersection graphs are Hamiltonian, and hence every $(v, K, 1)$-PBD with $\min (K) \geq 3$ admits an 
ordering in which pairs of consecutive blocks (of any size) intersect in exactly one point [30]. Recently, Case and Pike have shown that every $(v, K, \lambda)$-PBD, with $\lambda \geq 2$ and $\max (K) \leq \lambda \min (K)$, admits a cyclic ordering in which every pair of consecutive blocks intersects (perhaps in multiple points) [9]. On the other hand, M. Colbourn and Johnstone have dealt with the non-existence of configuration orderings for triple systems. In [20] they define a simple twofold triple system to be a $\operatorname{TTS}(v)$ having no non-trivial proper twofold subsystems and no repeated blocks. They present a simple TTS $(v)$ that does not admit an $A_{3}$-ordering (see Figure 1) and they imply that there exists a family of simple twofold triple systems having this property.

Cohen and Colbourn's work on configuration orderings stems from an erasurecorrection application. Many computer architectures connect a large number of disks together in an attempt to improve $\mathrm{I} / \mathrm{O}$ performance. A disk fails when some bits of the disk are deemed unreadable; an unreadable bit is called an erasure. Erasurecorrecting codes are designed to recover erased bits of data when the location of the erased bits is known. Given an erasure-correcting code, Cohen and Colbourn use orderings of the blocks STSs to represent orderings of disks that are either good or bad for system performance. Cohen and Colbourn have shown that for all admissible $v \geq 9 \ell-6, \ell \in \mathbb{N}$, there exists an $\operatorname{STS}(v)$ admitting a $(3 \ell, \ell)$-ordering [15]. Further, for $\ell \in \mathbb{N}$ and admissible $v \geq 81(\ell-1)+1$, every $\operatorname{STS}(v)$ admits a $(3 \ell, \ell)$-ordering [15]. They have also shown that for all admissible $v \geq 15$, there exists an $\operatorname{STS}(v)$ 
that is not $B_{5}$-orderable (see Figure 2) [15].

Configuration orderings are interesting in their own right and they also provide a useful framework in which to discuss other ordering concepts. In particular, we can frame questions about the existence of Ucycles or Gray codes in terms of the existence of a particular configuration ordering. We will see that at times this is not an enlightening shift in perspective, however, in many cases it has proved useful. As configuration ordering is an emerging area of study, few configurations have been investigated, and the investigation has focused on Steiner triple systems. We expand the known results regarding existence of $A_{1}$ - and $B_{1}$-cyclic orderings to all triple systems, as Cohen and Colbourn's results deal exclusively with STSs. The smallest configuration for which there are no known ordering results (direct or translated) is $A_{3}$ (see Figure 1). As mentioned earlier, we prove that there exists either a Ucycle of rank three or a 2-intersecting Gray cycle for the blocks of at least one TTS $(v)$ for each order $v \equiv 1,3,4,7(\bmod 12)$, with sporadic exceptions. This result implies the existence of an $A_{3}$-cyclic ordering for these designs. For triple systems with $\lambda=2$, this is a good general result, as the authors of [20] suggest that there exists an infinite family of $\operatorname{TS}(v, \lambda) \mathrm{s}$ (but only show the existence of one specific design) that do not admit such orderings. The question of existence of $A_{3}$-orderings remains open for $\operatorname{TS}(v, \lambda) \mathrm{s}$ with $\lambda \geq 3$. An $A_{4}$-ordering (see Figure 1 ) exists only for the trivial $(3,3, \lambda)$-BIBDs where $\lambda \geq 2$, thus the next configuration that is interesting and has not been investigated 
is $B_{2}-$ known as the hut configuration (see Figure 2). We prove that every $\operatorname{TS}(v, \lambda)$, $v \geq 137$, admits a hut-cyclic ordering.

Instead of investigating the existence of configuration orderings for configurations with more than three blocks, we choose to focus on small configurations by generalizing our study in two different ways. First we prove ordering results for $(v, k, 1)$-BIBDs where $k \geq 3$. Let $A_{1}^{\prime}$ denote a $(2 k, 2)$-configuration and let $B_{1}^{\prime}$ denote a $(3 k, k)$ configuration. We prove that every $(v, k, 1)$-BIBD, $v \geq 2 k^{2}+1$, admits an $A_{1}^{\prime}$-cyclic ordering and that every $(v, k, 1)$-BIBD, $v \geq 12 k^{2}+1$, admits a $B_{1}^{\prime}$-cyclic ordering. Let $B_{2}^{\prime}$ denote the configuration of three blocks with two blocks intersecting in a single point and the third disjoint. We prove that every $(v, k, 1)$-BIBD, $v \geq 18 k^{2}-6 k+1$, admits a $B_{2}^{\prime}$-cyclic ordering.

The second generalization we make is to the definition of configuration ordering itself. Let $\mathcal{C}$ be a set of configurations, each having $\ell$ blocks. A $\mathcal{C}$-ordering for a design is a listing of the blocks of the design such that every $\ell$ consecutive blocks form a configuration isomorphic to one of the configurations in $\mathcal{C}$. When the list is cyclic, the ordering is called a $\mathcal{C}$-cyclic ordering. To distinguish between the two definitions of configuration ordering, we will call $\mathcal{C}$-orderings generalized orderings, while a configuration ordering as defined by Cohen and Colbourn will be called a standard ordering. The definition of generalized configuration ordering allows us to discuss all block ordering results in a common language. For example, a Ucycle of 
rank two for a $(v, k, \lambda)$-BIBD is equivalent to a $\mathcal{C}$-cyclic ordering, where $\mathcal{C}$ is a set of eight different configurations on three blocks. We chose to focus on smaller sets. In particular, we consider the existence of $\left\{B_{4}, B_{5}\right\}$-orderings. $B_{4}$ and $B_{5}$ are called the 3-path and the triangle, respectively. We have two motivations for determining the existence of $\left\{B_{4}, B_{5}\right\}$-cyclic orderings of the blocks of designs. The first is that when $\lambda=1$, a $\left\{B_{4}, B_{5}\right\}$-cyclic ordering is exactly a 1-intersecting Gray cycle. That is, the $\left\{B_{4}, B_{5}\right\}$-cyclic ordering is a minimal change ordering for the blocks of a design because any two blocks from such a design intersect in at most one point. While results regarding the Hamiltonicity of the 1-block intersection graph have already proved the existence of such designs, a $\left\{B_{4}, B_{5}\right\}$-ordering has the additional property that no point stays on consecutive blocks of the list for "too long" (a Ucycle-like property). We prove that every cyclic $\operatorname{STS}(v), v \neq 3$, admits a $\left\{B_{4}, B_{5}\right\}$-cyclic ordering. Since for each admissible order there exists a cyclic $\operatorname{STS}(v)$, this result implies that for each admissible order $v \neq 3$, there exists an $\operatorname{STS}(v)$ that admits a $\left\{B_{4}, B_{5}\right\}$-cyclic ordering. This ordering is best possible in the sense that Cohen and Colbourn have shown that there exists a Steiner triple system of every admissible order that does not admit a $B_{5}$-ordering [15]. The second motivation for considering the existence of $\left\{B_{4}, B_{5}\right\}$ cyclic orderings relates to a design theoretic analogue of a graph theoretic concept. It is easy to show that if a graph is Eulerian, its line graph is Hamiltonian. Furthermore, it is easy to find an example proving that the converse of this statement is false. This 
leads to the following well-known theorem: a graph $G$ is Eulerian if and only if its line graph is Hamiltonian and no pair of consecutive edges in the Hamilton cycle represents a 3-claw in $G[58]$. The analogue of this theorem in design theory requires the block intersection graph. The block intersection graph of a design $S=(V, \mathcal{B})$ is a graph with vertices representing the blocks of $\mathcal{B}$ and two vertices, $B_{1}$ and $B_{2}$, connected if $B_{1} \cap B_{2} \neq \emptyset$. We associate the block intersection graph of a design with the line graph of a graph. By analogy to a graph, a design is Eulerian if and only if the block intersection graph is Hamiltonian and there exists a Hamilton cycle in the block intersection graph which does not pass through any 3-claws in the design. Note that this definition of Eulerian is not the same as one used in the context of hypergraphs [3]. The existence of Hamilton cycles in block intersection graphs has been settled for several large families of designs [2, 32] - are these designs Eulerian? That is, does there exist a Hamilton cycle in the block intersection graph having the required property? The restriction on the Hamilton cycle can easily be expressed using configuration ordering terminology by explicitly not allowing the claw configuration on three blocks to appear.

The remainder of this dissertation is organized as follows. Chapter 2 presents some basic terminology and results in design theory, in the process introducing symbols that will be used throughout the dissertation. Chapter 2 also provides background on Gray codes and Ucycles. In Chapter 3, we look more closely at the orderings for 
block designs considered in this dissertation. A survey of relevant known results in Gray codes, Ucycles and configuration orderings for designs is given. In particular, we translate results from other areas into the terminology of configuration ordering. The main body of new work appears in Chapters 4 and 5 . In Chapter 4 , we prove the existence of Gray codes and Ucycles for several infinite families of designs. In Chapter 5 , we discuss and prove the existence of several different configuration orderings (both standard and generalized). Finally, Chapter 6 summarizes our results and looks ahead to future work, introducing the groundwork for proving the existence of rank two Ucycles for BIBDs in general. We finish by posing some questions and proposing some conjectures. 


\section{Chapter 2}

\section{Background}

This chapter provides the background necessary for understanding Chapters $3-6$. We begin with a basic introduction to the designs and design theory concepts used throughout this dissertation. It is not required reading for someone with knowledge of design theory, however, we formalize the symbols and terminology that will be used throughout. Design theoretic definitions come from [54], unless otherwise indicated. The second half of the chapter deals with more advanced concepts, including configurations, Gray codes and Universal cycles. For a more detailed overview of these concepts and a history of major results in each area see $[19,26,47,12]$. Graph theoretic concepts will be defined as required. 


\subsection{Designs}

\subsubsection{Balanced Incomplete Block Designs}

In this section we define the designs that will be the focus of most discussion in the following chapters.

Definition 2.1.1. (Design) $A$ design, or set system, is a pair $(V, \mathcal{B})$ such that $V$ is a set of elements called points and $\mathcal{B}$ is a collection of non-empty subsets of $V$ called blocks.

Definition 2.1.2. $(t-(v, k, \lambda)$ Design) Let $t, v, k$ and $\lambda$ be positive integers such that $2 \leq t \leq k<v$.A $t-(v, k, \lambda)$ design is a set system $(V, \mathcal{B})$, such that the following properties hold: (1) $|V|=v$, (2) each block contains exactly $k$ points, and (3) every t-set of $V$ is contained in exactly $\lambda$ blocks.

When $t$, known as the strength of the design, is equal to two, a $t-(v, k, \lambda)$ design is called a balanced incomplete block design and is denoted $(v, k, \lambda)$-BIBD. Many of the results presented in this dissertation (both known and new) focus on a specific type of BIBD. Triple systems are $(v, k, \lambda)$-BIBDs having $k=3$. A triple system on $v$ points is denoted $\operatorname{TS}(v, \lambda)$. When $\lambda=1$, the triple system is called a Steiner triple system and is denoted $\operatorname{STS}(v)$. When $\lambda=2$, the triple system is called a twofold triple system and is denoted TTS $(v)$.

It is standard practice to let $b$ denote the number of a blocks in a given design, 
and to let $r_{x}$ represent the number of blocks in which the point $x$ appears. When every point of the design appears in the same number of blocks, simply denote this number by $r$.

Theorem 2.1.1. (See [54]) $A(v, k, \lambda)-B I B D, S=(V, \mathcal{B})$, has exactly

$$
b=\frac{v(v-1) \lambda}{k(k-1)}
$$

blocks. Moreover, each element of $V$ is contained in exactly

$$
r=\frac{(v-1) \lambda}{k-1}
$$

blocks.

The property represented by $\lambda$ is known as the index of the design. It is sometimes useful to express all five parameters of a BIBD, in which case we write $(v, b, r, k, \lambda)$ BIBD. A basic requirement for the existence of a BIBD is that $r$ and $b$ be integer. Another necessary condition for existence is Fisher's Inequality.

Theorem 2.1.2. (Fisher's Inequality, see [54]) In any $(v, b, r, k, \lambda)-B I B D, b \geq v$.

The design most commonly used to illustrate design theoretic concepts is the Fano plane. The Fano plane is a $(7,3,1)$-BIBD, also written STS(7). The design has $b=7$ and $r=3$. The Fano plane is represented in Figure 3. Each vertex is a point of the design and each line (including the center circle) represents a block of the design.

As we will often be working with triple systems, we note the following two existence theorems. 


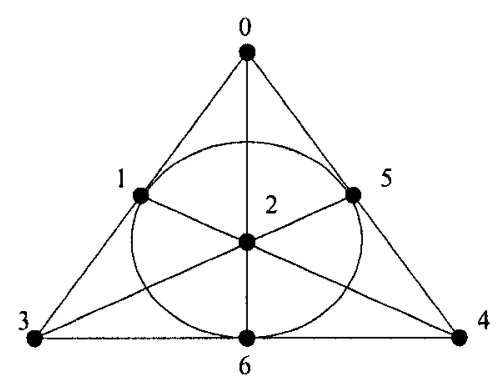

Figure 3: The $(7,3,1)$-design known as the Fano plane

Theorem 2.1.3. (See [19]) There exists an $S T S(v)$ if and only if $v \equiv 1,3(\bmod 6)$.

Theorem 2.1.4. (See [19]) There exists a TTS(v) if and only if $v \equiv 0,1(\bmod 3)$.

It is worthwhile to note the existence of some other special BIBDs.

Theorem 2.1.5. (See [54]) For every prime power $q \geq 2$, there exists a $\left(q^{2}+q+\right.$ $1, q+1,1)-B I B D$

The family of BIBDs defined in Theorem 2.1.5 are known as projective planes of order $\boldsymbol{q}$. Notice that the Fano plane is a projective plane of order 2. All projective planes are symmetric BIBDs.

Definition 2.1.3. (symmetric BIBD) A symmetric BIBD is one in which $b=v$ (or, equivalently, $r=k$ ).

One of the most important theorems regarding the existence of symmetric BIBDs is the Bruck-Ryser-Chowla Theorem. 
Theorem 2.1.6. (Bruck-Ryser-Chowla, see [54])

(i) If there exists a symmetric $(v, k, \lambda)-B I B D$ with $v$ even, then $k-\lambda$ is a perfect square.

(ii) If there exists a symmetric $(v, k, \lambda)$-BIBD with $v$ odd, then there exist integers $x, y, z$ (not all zero) such that $x^{2}=(k-\lambda) y^{2}+(-1)^{(v-1) / 2} \lambda z^{2}$.

Let $H$ be an abelian group of order $v$. For $h \in H$, the set $D+h=\{d+h: d \in D\}$ is known as a translate of $D$. A $(v, k, \lambda)$-difference set, $D$, is a $k$-set of elements from $H$ with the property that given any nonzero member $h \in H$, there are precisely $\lambda$ ordered pairs of elements in $D$ whose difference is $h$ [57]. The design obtained by developing a difference set $D$ in the group $H$ consists of all translates $\{D+h: h \in H\}$. This design is a symmetric $(v, k, \lambda)$-BIBD. An example of a $(7,3,1)$-difference set over $\mathbb{Z}_{7}$ is $\{1,2,4\}$. The development of difference sets into BIBDs can be generalized as follows. Suppose $D_{1}, \ldots, D_{n}$ are $k$-sets of elements of $H$. Let $\lambda_{i j}$ denote the number of ordered pairs of elements in $D_{j}$ with non-zero difference $h_{i} \in H$. If all sums $\sum_{j=1}^{n} \lambda_{i j}$ are equal, then developing these sets in $H$ yields a $\left(v, n v, n k, k, \sum_{j=1}^{n} \lambda_{1 j}\right)$-BIBD. The sets $D_{1}, \ldots, D_{n}$ are called supplementary difference sets [57]. For example, a $(9,18,8,4,3)$-supplementary difference set over $\mathbb{Z}_{9}$ is $\{\{1,2,3,5\},\{1,2,5,7\}\}$. Designs that come from difference sets have special properties. In particular, the relationship of the differences appearing in a difference set determines the automorphism group of the design. 


\subsubsection{Automorphisms of BIBDs}

In this section, we look at constructing BIBDs that have prescribed sets of automorphisms. The constructions are broken into two types based on the type of automorphism we are interested in: cyclic or rotational. First, we introduce some necessary terminology. An isomorphism from a design $\left(V_{1}, \mathcal{B}_{1}\right)$ to a design $\left(V_{2}, \mathcal{B}_{2}\right)$ is a bijective map $\phi: V_{1} \rightarrow V_{2}$ which maps $\mathcal{B}_{1}$ to $\mathcal{B}_{2}$. An automorphism of a BIBD is an isomorphism from the BIBD to itself. Let $V$ be a set of $v$ elements and let $\Gamma$ be a permutation group acting on $V$ (with operation composition). The set of all automorphisms of a BIBD forms a permutation group and any subgroup of the automorphism group is itself a permutation group. Suppose $(V, \mathcal{B})$ is a BIBD and suppose $\Gamma$ is a subgroup of its automorphism group. The orbit of a block $B \in \mathcal{B}$ with respect to $\Gamma$ is defined

$$
\operatorname{orb}_{\Gamma}(B)=\{\pi \cdot B: \pi \in \Gamma\}
$$

where $\pi$ operates on each element of $B$ independently. For some collection $\mathcal{B}^{\prime} \subseteq \mathcal{B}$,

$$
\mathcal{B}=\cup_{B \in \mathcal{B}^{\prime}}\left\{\operatorname{orb}_{\Gamma}(B)\right\}
$$

Let $\widehat{\mathcal{B}}$ be a set containing one block from each orbit; in group theoretic terms this is a set of orbit representatives. $\widehat{\mathcal{B}}$ is a set of base blocks, or starter blocks, for the BIBD under the action of $\Gamma$ because $\operatorname{or}_{\Gamma}(\widehat{\mathcal{B}})=\mathcal{B}$.

Let $V=\mathbb{Z}_{v}$ be the point set of a BIBD of order $v$. The design is called cyclic if its automorphism group (operating on the points of the system) contains the cyclic 
group of order $v$ as a subgroup. This cyclic group contains the automorphism

$$
\pi: i \longmapsto i+1 \quad(\bmod v)
$$

therefore, increasing every element of a block by one modulo $v$ results in another block of the design. While we will later work with cyclic $(v, k, \lambda)$-BIBDs with general $k$, most analysis of cyclic designs focuses on triple systems. The discussion in the following paragraph can be extended to base blocks of size $k$.

Let $V=\mathbb{Z}_{v}$ be the point set of a triple system of order $v$. All arithmetic will be carried out modulo $v$. The orbit of a pair $\{x, y\}, x, y \in V$, under the action of $\pi$ (defined above) is $\operatorname{orb}_{\pi}(\{x, y\})=\{\{x+i, y+i\}: 0 \leq i \leq v\}$. Orbits of pairs are separated into two classes based on their length. A full orbit is an orbit of length $v$. If $v$ is even, the orbit of $\{x, x \pm v / 2\}$ is of length $v / 2$; we call this a half orbit. We can specify an orbit of pairs uniquely by stating the minimum difference between the two elements. That is, the orbit of the pair $\{x, y\}$ is specified by $\min (|x-y|,|y-x|)$. This is called the minimum difference of a pair. Because an orbit of pairs can be specified by a single difference, the most logical set of orbit representatives of pairs is $\{\{0, d\}: 1 \leq d \leq\lfloor v / 2\rfloor\}$. Orbits of the triples in a cyclic triple system can be classified in a similar way. An orbit of triples is of length $v$ unless $v$ is a multiple of three. If $v$ is a multiple of three the orbit containing $\{0, v / 3,2 v / 3\}$ has length $v / 3$; such an orbit is called a short orbit. For each orbit of triples, consider the orbits of pairs associated to it. For example, the triple $\{x, y, z\}$ has associated to it the pair orbits represented 
by $\min (|x-y|,|y-x|), \min (|y-z|,|z-y|)$, and $\min (|x-z|,|z-x|)$. However, the orbits of pairs associated to a triple do not completely determine the orbit of triples. To represent an orbit of triples we use an object called a difference triple. Note that a difference triple is not the same as the difference sets defined above. Given a full orbit of triples, choose an orbit representative $\{0, a, b\}$ such that $a<b, a \leq v-b$ and $b-a \leq v-b$. The difference triple for this orbit is $(a, b-a, \min (b, v-b))$. In general, a triple $\left(d_{1}, d_{2}, d_{3}\right)$ is a difference triple modulo $v$ if and only if $d_{i} \leq v / 2$ and either $d_{1}+d_{2}+d_{3} \equiv 0(\bmod v)$ or $d_{1}+d_{2} \equiv d_{3}(\bmod v)$. To a short orbit of triples we associate $v / 3$, which is called a degenerate difference triple. A difference partition of a set is a partition of the set into difference triples.

In Chapter 4, we will work closely with cyclic BIBDs. When discussing cyclic BIBDs we will refer to the base blocks of the BIBDs. The terminology applied to orbits in the previous paragraph will also be used to describe the properties of base blocks. Suppose $\widehat{\mathcal{B}}$ is a collection of base blocks for a $(v, k, \lambda)$-BIBD. Given a base block $B \in \widehat{\mathcal{B}}$, define $\operatorname{Dev}(B)=\left\{x+B: x \in \mathbb{Z}_{v}\right\}$, where $x+B$ denotes the addition of $x$ to every point in $B$. We call the set $\operatorname{Dev}(B)$ the set of blocks developed from $B$. Equivalently, we say that $B$ generates the set $\operatorname{Dev}(B)$. If $B$ generates $v$ unique blocks, then $B$ is called a full orbit base block. If $B$ generates fewer than $v$ unique blocks, then $B$ is called a short orbit base block. A base block that generates exactly $v / k$ blocks is called a regular short orbit base block. Such a base block 
must be of the form $\{0, v / k, 2 v / k, \ldots,(k-1) v / k\}$. Note that the number of blocks generated by a base block must divide $v$. By convention a base block is assumed to include the zero point.

Cyclic triple systems are well-studied. The following theorems and lemmas present some relevant existence results.

Lemma 2.1.7. (See [57])

(i) Suppose $v \equiv 1(\bmod 6)$ and there is a difference partition of $S=\{1,2, \ldots$, $(v-1) / 2\}$, then there exists a cyclic $S T S(v)$.

(ii) Suppose $v \equiv 3(\bmod 6)$ and there is a difference partition of $S^{\prime}=\{1,2, \ldots$, $(v-1) / 2\} \backslash\{v / 3\}$, then there exists a cyclic $S T S(v)$.

Proof. Suppose $v \equiv 1(\bmod 6)$ and suppose $P$ is a difference partition of $S=$ $\{1, \ldots,(v-1) / 2\}$. Consider a difference triple $T=(x, y, z)$ in $P$. We will translate $T$ in order to obtain a triple containing zero. Some member of $T$ is congruent to the sum of the other two. Without loss of generality, suppose $z \equiv(x+y)(\bmod v)$. We associate $\{0, x, x+y\}$ to $T$ and set $\widehat{\mathcal{B}}=\{\{0, x, x+y\}:(x, y, z) \in P\}$. The triples formed by developing each base block of $\widehat{\mathcal{B}}$ in $\mathbb{Z}_{v}$ form a Steiner triple system.

Suppose $v \equiv 3(\bmod 6)$. The same construction is used, however, we add the blocks generated by $\{0, v / 3,2 v / 3\}$ - the base block associated to the degenerate difference triple $(v / 3)$. 
To illustrate, we construct an STS(15). Let $S^{\prime}=\{1,2,3,4,6,7\}$ and $P=$ $\{(1,3,4),(2,6,7)\}$. Then $\widehat{\mathcal{B}}=\{\{0,1,4\},\{0,6,13\},\{0,5,10\}\}$, where $\{0,5,10\}$ is associated to the degenerate difference triple. The STS(15) constructed from the difference partition $P$ is given in Table 1 .

$\begin{array}{lll}\{0,1,4\} & \{0,6,13\} & \{0,5,10\} \\ \{1,2,5\} & \{1,7,14\} & \{1,6,11\} \\ \{2,3,6\} & \{2,8,0\} & \{2,7,12\} \\ \{3,4,7\} & \{3,9,1\} & \{3,8,13\} \\ \{4,5,8\} & \{4,10,2\} & \{4,9,14\} \\ \vdots & \vdots & \\ \{14,0,3\} & \{14,5,12\} & \end{array}$

Table 1: An STS(15) constructed from the difference partition $P=\{(1,3,4),(2,6,7)\}$

The construction described in the proof of Lemma 2.1.7 need not be restricted to the case $\lambda=1$. For example, suppose we have the difference triples $\{(1,1,2),(2,2,3)$, $(1,3,3)\}$ over the group $\mathbb{Z}_{7}$. These triples induce a set of base blocks $\widehat{\mathcal{B}}=\{\{0,1,2\}$, $\{0,2,4\},\{0,3,6\}\}$ and these base blocks generate the TS(7,3) presented in Table 2.

$$
\begin{array}{lll}
\{0,1,2\} & \{0,2,4\} & \{0,3,6\} \\
\{1,2,3\} & \{1,3,5\} & \{1,4,0\} \\
\{2,3,4\} & \{2,4,6\} & \{2,5,1\} \\
\{3,4,5\} & \{3,5,0\} & \{3,6,2\} \\
\{4,5,6\} & \{4,6,1\} & \{4,0,3\} \\
\{5,6,0\} & \{5,0,2\} & \{5,1,4\} \\
\{6,0,1\} & \{6,1,3\} & \{6,2,5\}
\end{array}
$$

Table 2: A TS(7,3) constructed from the set of difference triples $\{(1,1,2),(2,2,3),(1,3,3)\}$ 
Theorem 2.1.8. (See [57]) There exists a difference partition of $S=\{1,2, \ldots$, $(v-1) / 2\}$ for all $v \equiv 1(\bmod 6)$, and a difference partition of $S^{\prime}=\{1,2, \ldots$, $(v-1) / 2\} \backslash\{v / 3\}$ for all $v \equiv 3(\bmod 6)$ except $v=9$.

Corollary 2.1.9. (See [57]) There exists a cyclic Steiner triple system of every possible order except 9.

A complete proof of this theorem can be found in [57]. The proof relies on answers to Heffter's first and second difference problems.

Heffter's first difference problem: Can $\{1,2, \ldots, 3 m\}$ be partitioned into a collection of $m$ difference triples modulo $6 m+1$ ?

The answer is yes and this enables us to form an $\operatorname{STS}(6 m+1)$. For example, if $v=18 s+1$ and $s \geq 2$, the difference partition of $S$ is given by

$$
\begin{aligned}
(3 r+1,4 s-r+1,4 s+2 r+2) & \text { for } r=0, \ldots, s-1, \\
(3 r+2,8 s-r, 8 s+2 r+2) & \text { for } r=0, \ldots, s-1, \\
(3 r+3,6 s-2 r-1,6 s+r+2) & \text { for } r=0, \ldots, s-1, \\
(3 s, 3 s+1,6 s+1) . &
\end{aligned}
$$

Heffter's second difference problem: Can $\{1,2, \ldots, 3 m+1\} \backslash\{2 m+1\}$ be partitioned into a collection of $m$ difference triples modulo $6 m+3$ ?

Again, the answer is yes and this enables us to form an $\operatorname{STS}(6 m+3)$. For example, 
if $v=18 s+3$ and $s \geq 1$, the difference partition of $S^{\prime}$ is given by

$$
\begin{aligned}
(3 r+1,8 s-r+1,8 s+2 r+2) & \text { for } r=0, \ldots, s-1, \\
(3 r+2,4 s-r, 4 s+2 r+2) & \text { for } r=0, \ldots, s-1, \\
(3 r+3,6 s-2 r-1,6 s+r+2) & \text { for } r=0, \ldots, s-1 .
\end{aligned}
$$

The existence of cyclic triple systems has been completely determined.

Theorem 2.1.10. (See [19]) There is a cyclic TS $(v, \lambda)$ if and only if:

(i) $\lambda \equiv 1,5(\bmod 6)$ and $v \equiv 1,3(\bmod 6),(v, \lambda) \neq(9,1)$,

(ii) $\lambda \equiv 2,10(\bmod 12)$ and $v \equiv 0,1,3,4,7,9(\bmod 12),(v, \lambda) \neq(9,2)$,

(iii) $\lambda \equiv 3(\bmod 6)$ and $v \equiv 1(\bmod 2)$,

(iv) $\lambda \equiv 4,8(\bmod 12)$ and $v \equiv 0,1(\bmod 3)$,

(v) $\lambda \equiv 6(\bmod 12)$ and $v \equiv 0,1,3(\bmod 4)$,

(vi) $\lambda \equiv 0(\bmod 12)$ and $v \geq 3$

Besides cyclic automorphisms, there are other types of automorphism that may appear in the automorphism group of a BIBD. A BIBD whose automorphism group contains a map which has one fixed point and $r$ cycles of the same length is called $\boldsymbol{r}$-rotational. A 1-rotational $(v, k, \lambda)$-BIBD is a design that has an automorphism with one fixed point and a cycle of length $v-1$. By convention the point set of a 
1-rotational design of order $v$ is $\mathbb{Z}_{v-1} \cup\{\infty\}$, with $\infty$ the fixed point, and so the cycle

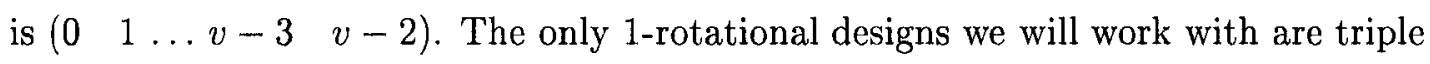
systems.

Theorem 2.1.11. (See [19]) There is a 1-rotational TS $(v, \lambda)$ if and only if:

(i) $\lambda=1$ and $v \equiv 3,9(\bmod 24)$,

(ii) $\lambda \equiv 1,5(\bmod 6)$ and $v \equiv 1,3(\bmod 6)$,

(iii) $\lambda \equiv 2,4(\bmod 6)$ and $v \equiv 0,1(\bmod 3)$,

(iv) $\lambda \equiv 3(\bmod 6)$ and $v \equiv 1(\bmod 2)$,

(v) $\lambda \equiv 0(\bmod 6)$ and $v \neq 2$.

\subsubsection{Pairwise Balanced Designs}

The concept of a BIBD can be generalized as follows.

Definition 2.1.4. (Pairwise Balanced Design) Let $v$ and $\lambda$ be positive integers and let $K \subseteq\{n \in \mathbb{Z}: n \geq 2\}$. A pairwise balanced design, $(v, K, \lambda)-P B D$, is a set system $(V, \mathcal{B})$ with the following properties: (1) $|V|=v$, (2) $|B| \in K$ and $|B|<v$ for all $B \in \mathcal{B}$, and (3) every pair of distinct points is contained in exactly $\lambda$ blocks.

Note that there need not be blocks of every size in $K$ in a $(v, K, \lambda)$-PBD. Below is an example of an $(11,\{3,5\}, 1)$-PBD. The points denoted by $\infty$ and $\infty_{i}, 1 \leq i \leq 5$, are artifacts of the construction method used. 


$$
\begin{array}{lll}
\left\{1,4, \infty_{1}\right\} & \left\{2,3, \infty_{1}\right\} & \left\{\infty, 0, \infty_{1}\right\} \\
\left\{2,0, \infty_{2}\right\} & \left\{3,4, \infty_{2}\right\} & \left\{\infty, 1, \infty_{2}\right\} \\
\left\{3,1, \infty_{3}\right\} & \left\{4,0, \infty_{3}\right\} & \left\{\infty, 2, \infty_{3}\right\} \\
\left\{4,2, \infty_{4}\right\} & \left\{0,1, \infty_{4}\right\} & \left\{\infty, 3, \infty_{4}\right\} \\
\left\{0,3, \infty_{5}\right\} & \left\{1,2, \infty_{5}\right\} & \left\{\infty, 4, \infty_{5}\right\} \\
& & \left\{\infty_{1}, \infty_{2}, \infty_{3}, \infty_{4}, \infty_{5}\right\}
\end{array}
$$

Table 3: An $(11,\{3,5\}, 1)-\mathrm{PBD}$

\subsubsection{Block Intersection Graphs}

A natural way to express relationships between blocks of a design is to create a graph having the blocks of the design as vertices. Let $[n]$ denote the set $\{1,2, \ldots, n\}$.

Definition 2.1.5. (I-block intersection graph) For $I \subseteq[k]$, the I-block intersection graph of a $(v, k, \lambda)-B I B D, S=(V, \mathcal{B})$, is the graph whose vertex set is $\mathcal{B}$ and whose edge set is $E=\left\{\left(B_{1}, B_{2}\right):\left|B_{1} \cap B_{2}\right| \in I, B_{1}, B_{2} \in \mathcal{B}\right\}$.

When $I=[k]$ the $I$-block intersection graph is simply called the block intersection graph. Denote the $I$-block intersection graph of a design $S=(V, \mathcal{B})$ by $G_{I}^{\mathcal{B}}$. Another graphical expression of a block design is the block coloured pair adjacency graph. The block coloured pair adjacency graph for a $(v, k, \lambda)$-BIBD $S=(V, \mathcal{B})$ is the multigraph with vertex set $V$ such that for each pair of distinct vertices $a$ and $b$, there is an edge between $a$ and $b$ of colour $B_{i}$ if $\{a, b\} \subseteq B_{i} \in \mathcal{B}$. Denote the block coloured pair adjacency graph by $G^{V}$. Notice that when $\lambda=1, G^{V}$ is simply a complete graph on $|V|$ vertices. 
We introduce a variation on $G^{V}$ for cyclic designs. The base block coloured pair adjacency graph for a cyclic design $S=(V, \mathcal{B})$, with base blocks denoted $\widehat{\mathcal{B}}$, is the multigraph with vertex set $V$ such that for each pair of distinct vertices $a$ and $b$, there is an edge between $a$ and $b$ of colour $B_{i}$ if the difference $b-a$ is in the base block $B_{i} \in \widehat{\mathcal{B}}$. Denote the base block coloured pair adjacency graph by $G_{b a s e}^{V}$. Given any cyclic design, $G_{b a s e}^{V}$ will use fewer colours than $G^{V}$.

\subsection{Configurations}

Recall that a $t-(v, k, \lambda)$ design is a set system $(V, \mathcal{B})$ such that the following properties hold: (1) $|V|=v,(2)$ each block contains exactly $k$ points, and (3) every $t$-set of distinct points is contained in exactly $\lambda$ blocks. A partial design is a set of elements $V$ and a set of blocks $\mathcal{B}$ such that every $t$-set occurs in at most $\lambda$ blocks. Configurations are partial designs which, although not stated in the definition, are defined assuming an underlying $t-(v, k, \lambda)$ design.

Definition 2.2.1. (n-line configuration) A n-line configuration is a collection of $n$ lines (or subsets) having the property that every t-element subset is contained in at most $\lambda$ lines.

Alternatively, one can specify the number of lines and points in a configuration.

Definition 2.2.2. (( $\boldsymbol{p}, \ell)$-configuration) $A(p, \ell)$-configuration is a configuration 
of $p$ points on $\ell$ lines.

The term line is used instead of block to conform with geometric terminology, however, we will use the two terms interchangeably. In this section, we assume that the blocks in each configuration are of size three, while in Chapters 3,4 and 5, we will talk about configurations in the generalized setting of PBDs. Names have been given to the configurations on blocks of size three, thus, given a configuration $C$ on blocks of size three, $C^{\prime}$ will denote the generalization of this configuration to blocks of size $k$. When there is some question as to how to generalize a given configuration the generalized version will be explicitly defined. Table 4 is a $(12,16)$-configuration. Note that the point 1 appears with $5,6,7,8,9,10,11,12$ but not with 2,3 or 4 .

$$
\begin{array}{llll}
\{1,5,12\} & \{1,7,10\} & \{2,4,9\} & \{1,6,8\} \\
\{3,4,10\} & \{3,7,12\} & \{4,5,6\} & \{5,7,8\} \\
\{2,8,11\} & \{1,9,11\} & \{2,10,12\} & \{2,6,7\} \\
\{3,5,11\} & \{6,9,10\} & \{4,11,12\} & \{3,8,9\}
\end{array}
$$

Table 4: A $(12,16)$-configuration

Configurations are often presented visually. For example, Figure 4 represents the two non-isomorphic $(7,3)$-configurations. Note that specifying the number of points and blocks of the configuration does not uniquely determine the configuration.

The study of configurations focuses on the presence or absence of given configurations in triple systems. For example, the number of pairwise non-isomorphic $n$-line configurations that can occur as blocks of a $\operatorname{TS}(v, \lambda)$ has been determined for 


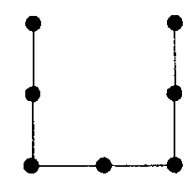

$\mathrm{B}_{4}$

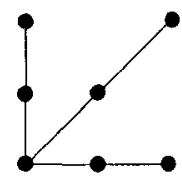

$\mathrm{B}_{3}$

Figure 4: The two non-isomorphic $(7,3)$-configurations

$1 \leq n \leq 3$ and arbitrary $\lambda$, and for $4 \leq n \leq 8$ when $\lambda=1$ [26]. The number of configurations of a certain type contained in an $\operatorname{STS}(v)$ has also been studied. For fixed $v$, non-isomorphic $\operatorname{STS}(v)$ s may contain different numbers of the same configuration. Grannell, Griggs and Mendelsohn have determined the number of times each 3-line configuration and the number of times each 4-line configuration appears in an $\operatorname{STS}(v)[27]$. Another question often asked regarding configurations is, given a $n$-line configuration $C$, can the blocks of an $\operatorname{STS}(v)$ be partitioned into copies of $C$ ? If $n \mid b$ such a decomposition is exact [26]. A decomposition that is not exact occurs when an $\operatorname{STS}(v)$ has an exact decomposition after the deletion of less than $n$ blocks. The seminal paper on decomposition of Steiner triple systems is [33]. The reader interested in decomposition will find the results of Horák and Rosa, and of Mullin, Poplove and Zhu (all contained in [26]) interesting.

The $n$-line configuration in which every pair of lines intersects in a single common point is called a $n$-star. We will use the following result in Chapter 4 .

Theorem 2.2.1. ([33]) Every STS(v) can be decomposed into 2-stars.

The 2-star is also denoted $A_{2}$. The 2-line configurations we will refer to in this 
dissertation are shown in Figure 1 (page 7), while the 3-line configurations are shown in Figure 2 (page 8).

Questions are also asked regarding the non-appearance of configurations in designs. For example, are there are triple systems that do not contain a particular type of configuration? Let $B(\lambda)$ be the set of values $v$ for which there exists a $\operatorname{TS}(v, \lambda)$ and let $C$ be a configuration. The avoidance set for $C$ and a given $\lambda$ is defined

$$
\Omega(C, \lambda)=\{v: v \in B(\lambda) \text { and } \exists \text { a } \operatorname{TS}(v, \lambda) \text { not containing } C\}
$$

One of the configurations most often considered in this context is the Pasch, shown in Figure 5. Designs avoiding the Pasch are called anti-Pasch or quadrilateral-free. The following theorem is the major result on avoidance.

Theorem 2.2.2. (See [28]) There exists an anti-Pasch $S T S(v)$ for all $v \equiv 1,3$ $(\bmod 6)$, except when $v=7$ or 13 .

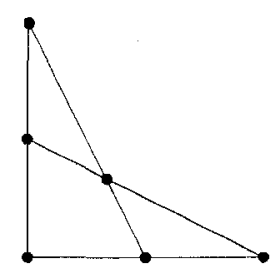

Figure 5: The Pasch configuration 


\subsection{Binary Gray Codes}

A common use of combinatorial algorithms is in the generation of combinatorial structures. The question of how to execute this generation efficiently is of particular importance. One way to make generation efficient is to ensure that successive elements are generated such that they differ in a small, pre-specified way. One of the earliest examples of such a process is Gray code generation. Introduced in 1953 in a pulse code communication system, Gray codes now have applications in areas as diverse as analog-to-digital conversion, coding theory, switching networks, logic circuits and experimental design (see [47] for an overview). Gray codes have been extensively studied over the past fifty years and, as a result, there are now many different types of Gray code.

Definition 2.3.1. (Binary Gray Code of Order n) A binary Gray code of order $n$ is a list of all $2^{n} n$-bit strings such that exactly one bit changes from one string to the next.

For the remainder of this section we will refer to binary Gray codes simply as Gray codes. A Gray code is cyclic if the change rule also holds from the last $n$-bit string to the first $n$-bit string. We will refer to cyclic Gray codes as Gray cycles. A binary string of length $n$ can be written as a vector $\left(a_{0}, a_{1}, \ldots, a_{n-1}\right)$, where $a_{i}=0$ or 1 , for $0 \leq i \leq n-1$. The characteristic vector for a subset of an $n$-set is a vector of length $n$ with a 1 in the $i^{\text {th }}$ position if $i$ is a member of the subset and 0 
if it is not. Therefore, a Gray code can be viewed as a listing of all subsets of a set $X=\left\{x_{0}, x_{1}, \ldots, x_{n-1}\right\}$. It is important to note that we label the characteristic vector positions 1 to $n$ from left to right (opposite to the standard method of labeling bit strings which we will employ in this section). Finally, by converting a binary string to its decimal representation, we obtain a third view of a Gray code. The code is a permutation of the set of the positive integers $\left\{0,1, \ldots, 2^{n}-1\right\}$.

\subsubsection{Standard Binary Reflected Gray Cycles}

In this section we look at what might be called the "original" Gray code. The standard binary reflected Gray cycle is defined as follows. Let $\Gamma_{n}$ denote the standard binary reflected Gray cycle of order $n \in \mathbb{N}$. Let $\Gamma_{0}$ be the empty list and let $\overline{\Gamma_{n-1}}$ denote the reversal of list $\Gamma_{n-1}$, where reversal refers to the order of the list rather than the order of the strings in the list. Let $x \Gamma_{n-1}, x \in \mathbb{N}$, indicate that $x$ is prepended to each string in the list $\Gamma_{n-1}$ and let ', denote concatenation of lists. For $n \geq 1$, the standard binary reflected Gray cycle has a simple recursive definition:

$$
\Gamma_{n}:=\left[0 \Gamma_{n-1}, 1 \overline{\Gamma_{n-1}}\right]
$$

Many Gray codes are created in this recursive manner; write the list of a smaller Gray code or its reversal multiple times, then prepend (or append) a fixed number of bits to each string. Table 5 presents two examples of standard binary reflected Gray cycles. 


$\begin{array}{cc}\Gamma_{2} & \Gamma_{3} \\ 00 & 000 \\ 01 & 001 \\ 11 & 011 \\ 10 & 010 \\ & 110 \\ & 111 \\ & 101 \\ & 100\end{array}$

Table 5: Standard binary reflected Gray cycles of orders 2 and 3, respectively

The expression of any binary Gray code can be compressed by specifying a start string (if not specified, assume it is the all zeros string) and then listing the bit position that changes as we move from one string to the next. This list of bit positions is called a transition sequence. When a code is cyclic this sequence includes the transition from the last to the first string. We adopt the convention of numbering the bit positions of a string from right to left, labeling the right-most bit the zero position. The transition sequence for $\Gamma_{3}$, shown in Table 5, is 01020102 (we eliminate the commas between bit positions when no confusion will result). Let $T_{n}$ denote the transition sequence for $\Gamma_{n}$, and let $T_{n}^{\prime}$ denote the sequence $T_{n}$ less the final bit transition. Let $T_{0}$ be the empty sequence, then, for $n \geq 1$, the transition sequence for the standard binary reflected Gray cycle can also be defined recursively:

$$
T_{n}:=T_{n-1}^{\prime}, n-1, \overline{T_{n-1}^{\prime}}, n-1=T_{n-1}^{\prime}, n-1, T_{n-1}^{\prime}, n-1
$$

The total number of times a bit position appears in a transition sequence is called 
its transition count. When a Gray code is cyclic, every bit position has an even transition count because every bit position must return to its starting value at completion of the cycle. For example, the transition counts for the bit positions of $\Gamma_{3}$ (a Gray cycle) are 4,2 and 2. Let $d(n)$ denote the number of different transition sequences (assuming a start string of all zeros) that define distinct Gray cycles of order $n$. A canonical transition sequence is one in which the first instance of bit position $i$ appears before the first instance of $i+1$. The transition sequence for $\Gamma_{3}$ in Table 5 is a canonical sequence. The only other canonical sequence for a Gray cycle of order 3 is 01210121 (note that this does not produce the standard binary reflected Gray cycle). Let $c(n)$ denote the number of canonical transition sequences of order $n$; then $d(n)=n ! \cdot c(n)[40]$. This is due to the fact that every permutation of the $n$ bit positions produces another transition sequence. It is interesting to note that $d(2)=2, d(3)=12$ and $d(4)=2688$. This is sequence A003042 in [51].

One of the most interesting and useful features of the standard binary reflected Gray cycle definition is that it provides an easy way to determine the location of a given bit string within the code without having to determine all strings appearing before it. The location of a given string within a code is called the rank of that string. It is also possible to unrank, that is, given a rank we can determine the string in that position of the code. Let

$$
\rho: \Gamma_{n} \rightarrow\left\{0,1, \ldots, 2^{n}-1\right\}
$$


be the function which takes a string in $\Gamma_{n}$ and maps it to its rank. Suppose the binary representation of $\rho(\gamma)$, for $\gamma \in \Gamma_{n}$, is $b_{n} b_{n-1} \ldots b_{1} b_{0}$. Then $b_{n}=0$ and $\rho(\gamma)=\sum_{i=0}^{n} b_{i} 2^{i}$. Let $\gamma$ be represented by $a_{n-1} a_{n-2} \ldots a_{0}$. Theorem 2.3 .1 defines the relationship between the binary representation of rank and the $n$-bit string of that rank for the standard binary reflected Gray cycle.

Theorem 2.3.1. (See [42]) Suppose that $n \geq 1$ is an integer and suppose that $b_{n} \ldots b_{0}$ and $a_{n-1} \ldots a_{0}$ are defined as above. Then, for $j=0,1, \ldots, n-1$,

$$
\begin{gathered}
a_{j} \equiv b_{j}+b_{j+1} \quad(\bmod 2), \\
b_{j} \equiv \sum_{i=j}^{n-1} a_{i} \quad(\bmod 2) .
\end{gathered}
$$

In the next subsection, we discuss binary Gray codes that exhibit additional uniformity properties.

\subsubsection{Balanced and Monotone Gray Codes}

A balanced Gray code is one in which the transition counts are as equal as possible across all bit positions. Note that the standard binary reflected Gray cycle of order $n$ is not at all balanced; the right-most bit changes $2^{n-1}$ times, whereas the left-most bit changes only twice (one of these being the return to the beginning of the code).

Definition 2.3.2. (Balanced Gray Code of order $n$ ) A balanced Gray code of order $n$ is a Gray code for which each transition count is $\left\lfloor\left(2^{n}-1\right) / n\right\rfloor$ or $\left\lceil\left(2^{n}-1\right) / n\right\rceil$. 


$\begin{array}{llll}00000 & 00110 & 10111 & 01010 \\ 10000 & 00010 & 10101 & 11010 \\ 11000 & 00011 & 10001 & 11011 \\ 11100 & 01011 & 11001 & 10011 \\ 11110 & 01001 & 11101 & 10010 \\ 11111 & 00001 & 01101 & 10110 \\ 01111 & 00101 & 01100 & 10100 \\ 01110 & 00111 & 01000 & 00100\end{array}$

Table 6: A balanced Gray cycle of order 5 (read column-wise)

Definition 2.3.3. (Balanced Gray Cycle of order $n$ ) Let $a=\left\lfloor 2^{n} / n\right\rfloor$ or $\left\lfloor 2^{n} / n\right\rfloor-1$, so that a is even. A balanced Gray cycle of order $n$ is a Gray code for which each transition count is a or $a+2$.

A totally balanced Gray cycle is one in which the transition counts are all equal. Wagner and West [56] have used a graph theoretic argument to show that totally balanced Gray cycles exist when $n$ is a power of two. In [4], Bhat and Savage show that balanced Gray cycles exist for all $n \in \mathbb{N}$. Table 6 shows an example of a balanced Gray cycle. In 1980, Vickers and Silverman [55] proposed a heuristic to compute balanced Gray codes. The idea is to take any Gray code of order $n$, remove a block of strings from the end of the code and insert that block between two strings in the remainder of the list, choosing the insertion point so that the resulting list is also a Gray code but with improved balance. While the algorithm performed well for $n=5,6,7,8$, there is no proof as to the efficacy of this approach in general.

Define the weight of a binary string to be the number of one bits it contains. It 


$\begin{array}{llll}00000 & 11000 & 01010 & 11110 \\ 00001 & 10000 & 01011 & 11100 \\ 00011 & 10001 & 01001 & 11101 \\ 00010 & 10101 & 01101 & 11001 \\ 00110 & 10100 & 00101 & 11011 \\ 00100 & 10110 & 00111 & 10011 \\ 01100 & 10010 & 01111 & 10111 \\ 01000 & 11010 & 01110 & 11111\end{array}$

Table 7: A monotone Gray code of order 5 (read column-wise)

is not possible to have a Gray code with strings listed by non-decreasing weight, but, it is possible to run through weight levels two at a time. Let $v_{i}$ represent a binary string of length $n$, and let $w\left(v_{i}\right)$ denote the weight of string $v_{i}$.

Definition 2.3.4. (Monotone Gray Code of order $\boldsymbol{n}$ ) Suppose $V=v_{0}, v_{1}, \ldots$, $\ldots, v_{2^{n}-1}$ is a Gray code of order $n . V$ is monotone if $w\left(v_{k}\right) \leq w\left(v_{k+2}\right)$ for $0 \leq k \leq$ $2^{n}-3$. That is, for all $0 \leq i<j<n$, consecutive strings of weight $i$ and $i+1$ precede those of weight $j$ and $j+1$.

Monotone Gray codes have applications to interconnection networks and also provide some insight into the Middle Levels Problem [48]. Savage and Winkler have proved, by an elegant construction, that there exists a monotone Gray code for each order $n \in \mathbb{N}$ [48]. Table 7 shows a monotone Gray code of order 5 . 


\subsection{Combinatorial Gray Codes}

The notion of a Gray code can be extended to combinatorial objects other than binary strings. Such listings have come to be known as combinatorial Gray codes. In this section we look at Gray codes for $k$-subsets of $n$-sets and Gray codes for permutations of $n$-sets.

\subsection{1 $k$-subsets of $n$-sets}

There are several ways to represent $k$-subsets of an $n$-set. For example, if we are considering 4 -subsets of $\{1,2, \ldots, 10\}$ we have the following representations of the subset $\{2,5,7,4\}:(1)$ unordered: $\{2,5,7,4\},(2)$ ordered: $(2,4,5,7)$, and (3) characteristic vector: $(0,1,0,1,1,0,1,0,0,0)$.

We are interested in listing all $k$-subsets of an $n$-set such that consecutive subsets differ in a small way. One such listing is a minimal change ordering. A list is in minimal change order if consecutive subsets differ by the deletion of one element and the insertion of another. In Section 2.3 we saw that there exists a binary Gray code of order $n$, for all $n \in \mathbb{N}$. Considering the $n$-bit strings of a binary Gray code to be characteristic vectors, we have a minimal change listing for all subsets of $[n]=\{1,2, \ldots, n\}$ (here the deletion or the insertion, but not both, may be the null element). Therefore, from a binary Gray code of order $n$, we can obtain a listing of all $k$-subsets of $[n]$, represented by characteristic vectors, simply by deleting all 
vectors except those of weight $k$. Denote such a list, obtained from the standard binary reflected Gray cycle, by $\Gamma_{n, k}$. The following result is due to Bitner et al.

Theorem 2.4.1. ([5]) Successive binary vectors in $\Gamma_{n, k}$ differ in exactly two bit positions.

While this is a surprising and nice feature of the standard binary reflected Gray cycle, in most cases we would rather not generate a complete binary Gray cycle and then delete most strings to obtain the Gray code for $k$-subsets of an $n$-set. The following sections discuss methods for directly generating minimal change lists containing all $k$-subsets of an $n$-set. Denote the set of $\left(\begin{array}{l}n \\ k\end{array}\right) k$-subsets of $[n]$ by $\mathcal{S}_{k}^{n}$. As with binary Gray codes, once we have established that the subsets of $\mathcal{S}_{k}^{n}$ can be listed in a minimal change order, additional constraints will be added to the definition of minimal change so that the elements allowed to change from one subset to the next are restricted.

\section{Revolving Door}

The most common method for generating minimal change listings for the $k$-subsets of $[n]$ is the revolving door algorithm. The revolving door ordering is based on Pascal's identity:

$$
\left(\begin{array}{l}
n \\
k
\end{array}\right)=\left(\begin{array}{l}
n-1 \\
k-1
\end{array}\right)+\left(\begin{array}{c}
n-1 \\
k
\end{array}\right)
$$

In the language of $k$-subsets of $[n]$, this identity represents the fact that the collection of $k$-subsets of an $n$-set can be partitioned into two disjoint sub-collections: the subsets 
that contain $n$ and the subsets that do not contain $n$. Let $A_{n, k}$ denote the revolving door ordering for $\mathcal{S}_{k}^{n}$. For $k=0, A_{n, 0}=[\emptyset]$ and for $k=n, A_{n, n}=[\{1,2, \ldots, n\}]$. Let $A_{n-1, k-1} \cup\{n\}$ denote the list $A_{n-1, k-1}$ with the element $n$ added to each set and let $\overline{A_{n, k}}$ denote the list $A_{n, k}$ in reverse order. For $0<k<n$,

$$
A_{n, k}:=\left[A_{n-1, k}, \overline{A_{n-1, k-1}} \cup\{n\}\right]
$$

Theorem 2.4.2. (See [42]) For any integers $k$ and $n$ such that $1 \leq k \leq n$, the list $A_{n, k}$ is a minimal change listing for $\mathcal{S}_{k}^{n}$.

Table 8 gives two examples of minimal change listings for $k$-subsets of [5] obtained using the revolving door algorithm.

$\begin{array}{ll}123 & 1234 \\ 134 & 1245 \\ 234 & 2345 \\ 124 & 1345 \\ 145 & 1235 \\ 245 & \\ 345 & \\ 135 & \\ 235 & \\ 125 & \end{array}$

Table 8: Revolving door lists for 3-subsets of [5] and 4-subsets of [5], respectively

\section{Strong Minimal Change}

The following property is known to Wilf as strong revolving door [59] and to others as strong minimal change [24]. A strong minimal change listing for $k$-subsets of 
$[n]$ is a minimal change listing with the additional restriction that two consecutive subsets, when written in sorted order, may differ only in a single component. That is, if the $i^{\text {th }}$ element of an ordered set leaves, then the new element must also fall in the $i^{\text {th }}$ position of the new ordered set. Eades and McKay [24] give a recursive definition for strong minimal change lists. Let $L_{n, k}$ denote a listing of $\mathcal{S}_{k}^{n}$ for which the strong minimal change condition is satisfied. For $k=0, L_{n, 0}=[\emptyset]$, for $k=1$, $L_{n, 1}=[(1),(2), \ldots,(n)]$, and for $k=n, L_{n, n}=[(1,2, \ldots, n)]$. Let $L_{n, k}+x$ denote the list where $x \in \mathbb{N}$ has been added to each entry of each ordered subset in the list $L_{n, k}$ and let $\left(x, L_{n, k}\right)$ denote the list of ordered subsets $L_{n, k}$ with $x$ joined as the first entry of each ordered subset. For $1<k<n$,

$$
L_{n, k}:=\left[\left(1,2, L_{n-2, k-2}+2\right),\left(1, \overline{L_{n-2, k-1}}+2\right),\left(L_{n-1, k}+1\right)\right]
$$

Table 9 gives an example of a strong minimal change listing.

$\begin{array}{llll}(1,2,3) & (1,4,6) & (2,3,4) & (2,4,5) \\ (1,2,4) & (1,4,5) & (2,3,5) & (3,4,5) \\ (1,2,5) & (1,3,5) & (2,3,6) & (3,4,6) \\ (1,2,6) & (1,3,6) & (2,5,6) & (3,5,6) \\ (1,5,6) & (1,3,4) & (2,4,6) & (4,5,6)\end{array}$

Table 9: Strong minimal change list for 3-subsets of [6] (read column-wise) 


\section{Adjacent Change}

An even stronger restriction on the listing of ordered $k$-subsets of $[n]$ is to require that consecutive ordered subsets differ only in a single component and that the change be an increase or decrease by one. This property is known as adjacent change due to the fact that if the $k$-subsets are in characteristic vector representation this restriction requires that consecutive vectors be of the form $\left(\ldots, x_{i}, x_{i+1}, 1,0, x_{i+4}, \ldots\right)$ and $\left(\ldots, x_{i}, x_{i+1}, 0,1, x_{i+4}, \ldots\right)$. This change may be referred to as a transposition of bits in the binary vectors.

The adjacent change requirement is so restrictive that it is not always possible to find a list satisfying the property. Eades et al. [23], and independently Buck and Wiedemann [7], have shown that such a listing exists only if $k=0,1, n-1, n$ or if $n$ is even and $k$ is odd. Ruskey proves this result in [45] by presenting a construction algorithm.

Since a strong minimal change listing is always possible but an adjacent change listing is not, it is reasonable to look for a restriction that falls between these two and for which it is always possible to construct a listing. Independently, Chase [11] and Ruskey [46] have proved that it is always possible to create a list with a relaxed form of the adjacent change property. Let two distinct binary vectors be two-close if they differ by a transposition of two bits that are either adjacent or have a single zero between them. In [46], Ruskey provides a recursive construction for such a listing. 


\subsubsection{Permutations}

In this section, we are interested in listing all $n$ ! permutations of an $n$-set such that consecutive permutations differ in a small way. A minimal change listing of permutations is a list in which consecutive permutations differ in exactly two positions. That is, one permutation can be obtained from the other by a single transposition of elements. It is always possible to create such a list [50], so it is natural to restrict the type of transpositions allowed and ask if it is possible to list all permutations such that consecutive permutations differ by an allowed transposition.

The most common method for generating a minimal change listing of permutations is the Trotter-Johnson algorithm. The list generated by this algorithm has the property that the positions in which consecutive permutations differ are adjacent. This is equivalent to saying that the set of allowed transpositions is $\left\{\left(\begin{array}{l}12 \\ 2\end{array}\right),(23), \ldots\right.$, $(i(i+1)), \ldots,((n-1) n)\}$. It is important to recognize that the transpositions act on the positions of an $n$-tuple. That is, $(i i+1)$ swaps the symbols in positions $i$ and $i+1$ in a given $n$-tuple; it does not swap the values $i$ and $i+1$. As with many combinatorial Gray code constructions, the Trotter-Johnson algorithm is recursive. Suppose $\mathcal{T}_{n-1}=\left[\pi_{0}, \pi_{1}, \ldots, \pi_{(n-1) !-1}\right]$ is a minimal change listing for the $(n-1)$ ! permutations of $[n-1]$. Form a new list by repeating each permutation in the list $n$ times, i.e. $\left[\pi_{0}, \ldots, \pi_{0}, \pi_{1}, \ldots, \pi_{1}, \ldots, \pi_{(n-1) !-1}, \ldots, \pi_{(n-1) !-1}\right]$. Now insert element $n$ into each of the $n$ copies of $\pi_{i}$ as follows. If $i$ is even, first place $n$ after the element in 
position $n-1$, then after the element in position $n-2$, and so on until $n$ is inserted at the beginning of the last $\pi_{i}$. If $i$ is odd, proceed with insertion in the opposite order. The resulting list is $\mathcal{T}_{n}$. Table 10 shows three Trotter-Johnson listings. The value $n$ is in bold to illustrate the pattern of insertion.

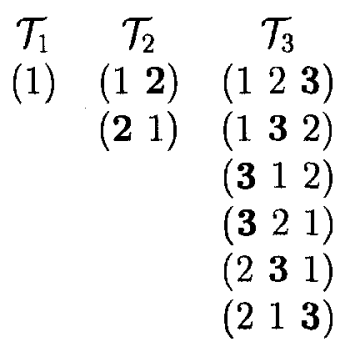

Table 10: Trotter-Johnson listings for permutations of order 1,2, and 3

The Trotter-Johnson construction shows that it is possible to list all permutations of $[n]$ such that each permutation can be obtained from the previous by the transposition of adjacent elements. Kompel'maker and Liskovets [41], and independently Slater [50], have shown that it is possible to list all permutations of $[n]$ such that each permutation can be obtained from the previous by a single transposition from an arbitrary fixed basis of transpositions for $S_{n}$ - the symmetric group on $n$ elements. The proof of Kompel'maker and Liskovets is technical and involves building a transition sequence of transpositions for $n+1$ from a sequence of transpositions for $n$. Slater takes a graph theoretical approach to the question which simplifies the proof. 


\subsection{De Bruijn Sequences}

De Bruijn sequences are a type of Gray code in which successive strings differ not in a single bit position but in a small structural way. A de Bruijn sequence is a listing of all $n$-bit stings such that successive strings differ by a shift one position left (dropping the first element) followed by the introduction of a new last element. This allows us to compress the list of all $n$-bit strings into a single cyclic list of $2^{n}$ bits.

Definition 2.5.1. (de Bruijn Sequence of Order $n$ ) A de Bruijn sequence of order $n$ is a circular binary sequence of length $2^{n}$ in which every $n$-bit string appears as a contiguous subsequence.

Figure 6 represents a de Bruijn sequence of order 4. De Bruijn sequences exist for all positive integers $n$. The standard proof involves first defining a de Bruijn digraph and then showing that such a graph has an Euler cycle. An Euler cycle is a cycle that uses each edge of a graph exactly once. Define the de Bruijn digraph of order $\boldsymbol{n}$, denoted $\mathcal{G}_{n}$, as follows. Let the vertices of the digraph be the $(n-1)$-bit strings. Join vertex $v=x_{0} x_{1} \ldots x_{n-2}$ by directed edges to vertices $v_{0}=x_{1} \ldots x_{n-2} 0$ and $v_{1}=x_{1} \ldots x_{n-2} 1$. Note that there are exactly $2^{n}$ edges in this digraph and that there exists an edge corresponding to each $n$-bit string - the string $x_{0} x_{1} \ldots x_{n-1}$ corresponds to the edge $\left(x_{0} x_{1} \ldots x_{n-2}, x_{1} \ldots x_{n-2} x_{n-1}\right)$. A de Bruijn sequence of order $n$ is equivalent to an Euler cycle in $\mathcal{G}_{n}$. Figure 7 shows the de Bruijn digraph of order 4. 


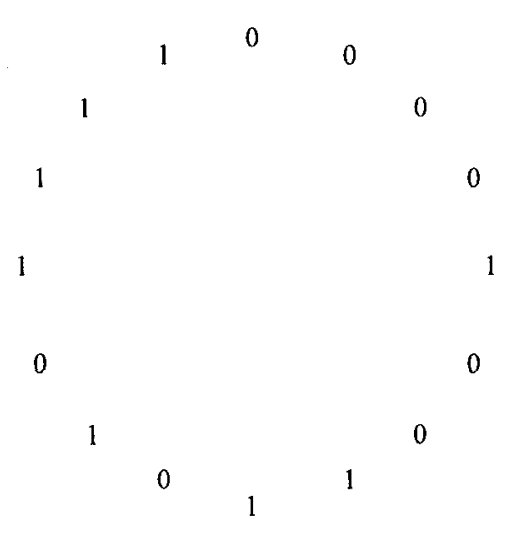

Figure 6: A de Bruijn sequence of order 4

A 2-in 2-out graph is a digraph in which every vertex has in-degree two and out-degree two. A strongly connected digraph is a digraph in which there is a directed path between any two vertices. The de Bruijn digraph of order $n$ is a strongly connected 2-in 2-out graph. The following well-known theorem implies that the de Bruijn digraph of order $n$ is Eulerian and consequently, that a de Bruijn sequence of order $n$ exists for all positive integers $n$.

Theorem 2.5.1. (See [6]) Let $D$ be a strongly connected digraph. $D$ has a directed Euler cycle if and only if the in-degree of each vertex equals its out-degree.

An interesting method for constructing de Bruijn sequences is due to Martin [43]. Given a set of $r$ different symbols, consider the $r^{n}$ arrangements of $n$ symbols with repetitions of the symbols allowed. Can a sequence of these symbols be constructed such that each of these $r^{n}$ arrangements is found exactly once as a subsequence of $n$ consecutive symbols in this sequence? Martin's algorithm proves, by construction, 


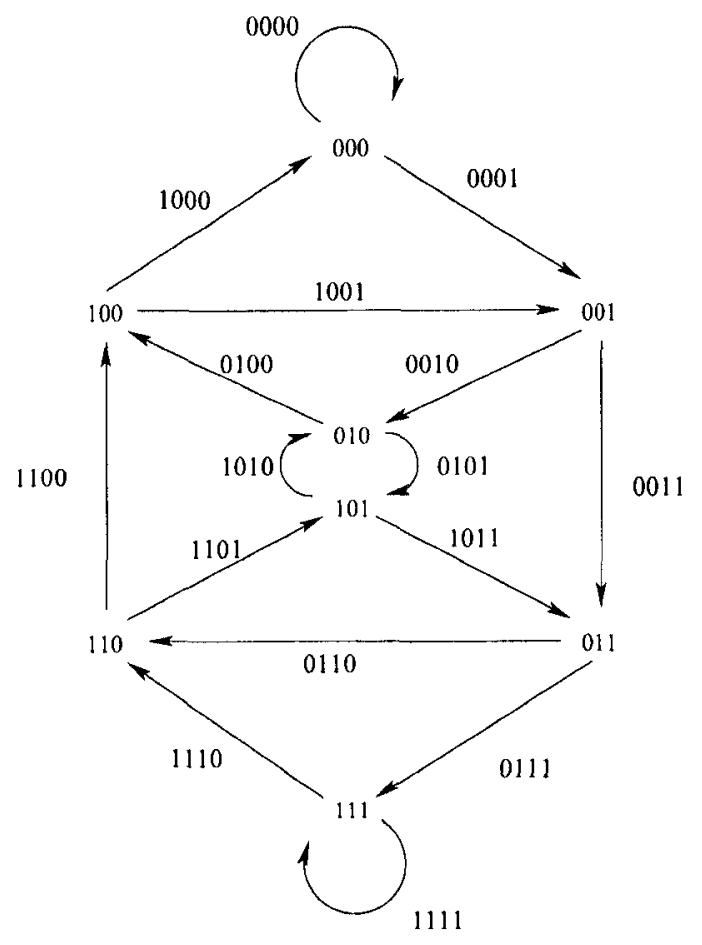

Figure 7: The de Bruijn digraph of order 4

that this question can be answered to the affirmative. The steps of the algorithm are listed below.

(1) Denote the $r$ different symbols $e_{0}, e_{1}, \ldots, e_{r-1}$.

(2) Each of the first $n-1$ symbols of the constructed sequence $S$ is chosen equal to $e_{0}$.

(3) The symbol $a_{m-1}$ to be added to the sequence $S$

$$
a_{0} a_{1} \ldots a_{n-2} a_{n-1} \ldots a_{m-n} \ldots a_{m-2}
$$


where $a_{0}=a_{1}=\ldots=a_{n-2}=e_{0}$ and $m-1 \geq n$, is the $e_{i}$ with the greatest subscript consistent with the requirement that the subsequence $a_{m-n} \ldots a_{m-2} a_{m-1}$ not duplicate a previously occurring subsequence of $n$ symbols in (2.5.1).

(4) Step 3 is first applied for $m=n$ (in which case $a_{m-1}=a_{n-1}=e_{r-1}$ ) and is then applied repeatedly until further application is impossible.

For the binary alphabet, this algorithm produces a de Bruijn sequence. In this case, the construction method amounts to applying the rule: start with $n-1$ zeros, then add a one as long as the new $n$-bit string is not a repeat of one already constructed; otherwise, add a zero.

De Bruijn sequences are named after N. de Bruijn who published a paper on their existence in 1946. However, these sequences were first discovered, and their existence proved, by Flye-Sainte Marie in 1894. De Bruijn sequences are also known as full length nonlinear shift register cycles. Various algorithms for determining at least one or all possible de Bruijn sequences for a given $n$ are presented in [25].

It is possible to generate a de Bruijn sequence of order $n$ from a de Bruijn sequence of order $n-1$. This idea is reminiscent of the recursive Gray code constructions discussed in Sections 2.3 and 2.4. Define an onto map between the set of $2^{n}$ binary strings of length $n$, denoted by $V_{n}$, and the set of $2^{n-1}$ binary strings of length $n-1$, 
denoted by $V_{n-1}$, as follows:

$$
\begin{aligned}
f: V_{n} & \longrightarrow V_{n-1} \\
\left(\mathbf{a}=a_{0} a_{1} \ldots a_{n-1}\right) & \longrightarrow\left(\mathbf{b}=b_{0} b_{1} \ldots b_{n-2}\right)
\end{aligned}
$$

where $f(\mathbf{a})=\mathbf{b}$ if and only if $b_{i}=a_{i}+a_{i+1}$ for $i=0,1, \ldots, n-2$ [25]. This relation implies that given an $(n-1)$-tuple $\mathbf{b}$ in $V_{n-1}$ and a single bit of a, say $a_{0}$, the next $n-1$ bits of a can be uniquely determined using the equation $a_{i+1}=a_{i}+b_{i}$, for $i=0,1, \ldots, n-2$, or equivalently, $a_{i+1}=a_{0}+b_{0}+b_{1}+\ldots+b_{i}$.

Let $\mathcal{C}$ be a de Bruijn sequence of order $n-1$. In this paragraph we abuse the definition of $f$ by discussing the application of $f$ to sequences of length $2^{n}$ rather than strings of length $n$. That is, if $t_{0}, t_{1}, \ldots, t_{2^{n}-1}$ is the image under $f$ of $s_{0}, s_{1}, \ldots, s_{2^{n}-1}$, then $t_{i}=s_{i}+s_{i+1}$, for $i=0,1, \ldots, 2^{n}-1$, with subscript addition modulo $2^{n} \cdot \mathcal{C}$ is the image under $f$ of two sequences in $V_{n}$; obtained by choosing $a_{0}=0$ or $a_{0}=1$. Denote the pre-image of $\mathcal{C}$ having $a_{0}=0$ by $\mathcal{C}_{0}$ and denote the pre-image of $\mathcal{C}$ having $a_{0}=1$ by $\mathcal{C}_{1}$. Clearly the bit-wise sum modulo 2 of $\mathcal{C}_{0}$ and $\mathcal{C}_{1}$ is the all ones string. We can join $\mathcal{C}_{0}$ and $\mathcal{C}_{1}$ at a common $(n-1)$-bit string to create a de Bruijn sequence of order $n$. The two sequences must share at least one common $(n-1)$-bit string. To see this, consider the all ones string of length $(n-1)$ in $\mathcal{C}$. The pre-image of this string under $f$ is either the $n$-bit string of alternating zeros and ones starting with 0 or the $n$-bit string of alternating zeros and ones starting with 1 ; each of these strings appears in one of $\mathcal{C}_{0}$ and $\mathcal{C}_{1}$, therefore, the $(n-1)$-bit string of alternating 
zeros and ones starting with 0 appears in both sequences. Note that we can think of each $\mathcal{C}_{i}$ sequence as a cycle. Each of the $2^{n-1}$ strings of length $n$ appearing in $\mathcal{C}_{i}$, for $i \in\{0,1\}$, are distinct and each sequence contains a distinct set of strings (otherwise $\mathcal{C}$ would contain a repeated $(n-1)$-bit string). To create a de Bruijn sequence of order $n$, splice one cycle into the other by writing the first cycle up to and including a common $(n-1)$-tuple, then writing the second cycle starting from the common $(n-1)$-tuple and cycling around to write the full cycle, finishing again with the common $(n-1)$-tuple, and finally completing the first cycle. For example, $\mathcal{C}=00011101$ is a de Bruijn sequence of order 3 with pre-images $\mathcal{C}_{0}=00001011$ and $\mathcal{C}_{1}=11110100$. We have a choice of where to splice the two cycles together as they have several length three subsequences in common. Arbitrarily choose the common sequence 101, which yields 0000101001111011 - a de Bruijn sequence of order 4.

\subsection{Universal Cycles}

Universal cycles are a generalization of de Bruijn sequences to other families of combinatorial objects. The definition was proposed by Chung, et al. in 1992 [12].

Definition 2.6.1. (Universal Cycle) Let $\mathcal{F}_{n}$ be a family of combinatorial objects of 'rank' $n$ and let $m=\left|\mathcal{F}_{n}\right|$. Assume each $F \in \mathcal{F}_{n}$ is specified by some sequence $\left\langle x_{0}, x_{1}, \ldots, x_{n-1}\right\rangle$, where, for $0 \leq i \leq n-1, x_{i} \in A$ for some fixed alphabet A. $\mathbf{U}=a_{0}, a_{1}, \ldots, a_{m-1}$ is a Universal cycle (or Ucycle) for $\mathcal{F}_{n}$ if $\left\langle a_{i+1}, \ldots, a_{i+n}\right\rangle$, 
$0 \leq i<m$, runs through each element of $\mathcal{F}_{n}$ exactly once, where index addition is performed modulo $\mathrm{m}$.

Note that the term rank does not refer to the size of each combinatorial object, but to the size of its representative. As an illustrative example, a de Bruijn sequence of order $n$ is a Ucycle for the family of binary strings of length $n$, so $\mathcal{F}_{n}=\left\{\left(x_{0}, \ldots, x_{n-1}\right): x_{i} \in\{0,1\}, 0 \leq i \leq n-1\right\}$ and $m=2^{n}$. In this case each binary vector represents itself, however, for other families we will see that the representative of each object may be different from the object and may not be the same size as the object. The next three sections review the literature on Ucycles for permutations, subsets and partitions.

\subsubsection{Permutations}

We divide our discussion of Ucycles for permutations into two parts: (1) $k$-permutations of an $n$-set where $k<n$, and (2) $n$-permutations of an $n$-set. By the set of $k$ permutations of an $n$-set we mean all $k !\left(\begin{array}{c}n \\ k\end{array}\right)$ arrangements of $k$-symbols from $[n]$, not permutations in the sense of the symmetric group. Thus 12 and 21 are distinct elements and we write these elements without brackets to avoid confusion. As the existence of Ucycles for $k$-permutations of $[n]$ has been completely solved, we present that result first.

The existence of Ucycles for $k$-permutations of $[n]$ was proved by Jackson in [39]. 
The proof is relatively easy as it employs a transition digraph. We have seen transition digraphs already - the de Bruijn digraph is an example of one. In general, a transition digraph for a family of combinatorial objects of order $n$ is a graph where vertices represent the objects of order $n-1$ and where each edge represents one of the objects of order $n$. Define the transition digraph for $k$-permutations of $[n]$, denoted $T_{n, k}$, as follows. The vertices are $(k-1)$-permutations of $[n]$. A directed edge $(u, v)$ exists if the $k-2$ length tail of vertex $u$ is the same as the $k-2$ length head of vertex $v$ and the head element of vertex $u$ is not equal to the tail element of vertex $v$, i.e. $u=x_{0} x_{1} \ldots x_{k-2}$ and $v=x_{1} \ldots x_{k-2} x_{k-1}, x_{0} \neq x_{k-1}$. The edge $(u, v)$ represents the permutation $x_{0} x_{1} \ldots x_{k-1}$, which is why it is necessary that $x_{0}$ be different from $x_{k-1}$. Figure 8 shows the transition digraph for 3-permutations of [4]. Notice that the edge $(10,02)$ exists but the edge $(02,20)$ does not.

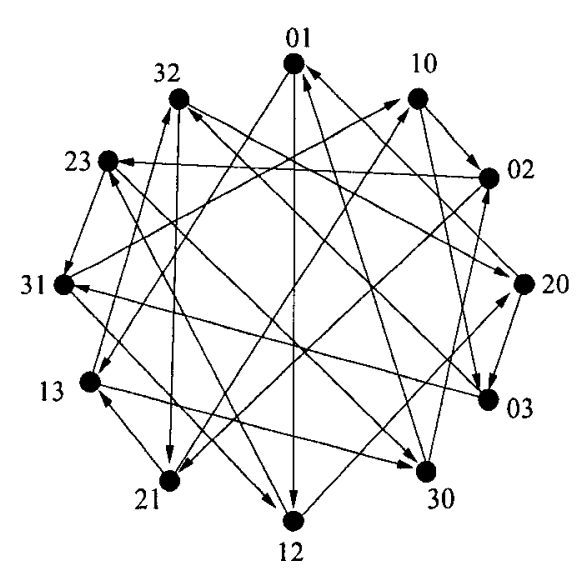

Figure 8: The transition digraph for 3-permutations of [4]

Theorem 2.6.1. ([39]) For every integer $k \geq 3$ and every integer $n \geq k+1$, there 
exists a Ucycle for the $k$-permutations of $[n]$.

We present the proof in full to illustrate the methods often employed in establishing the existence of Ucycles and to clarify some of Jackson's statements. We will require the following lemma which is stated but not proved by Jackson. For completeness we present the proof here. Define a cyclic rotation of a $t$-permutation to be a shift of $s<t$ contiguous elements from the front of the permutation to the end. That is, $i_{s} i_{s+1} \ldots i_{t-2} i_{t-1} i_{0} i_{1} \ldots i_{s-1}$ is a cyclic rotation of $i_{0} i_{1} \ldots i_{t-2} i_{t-1}$. Let $v=v_{0} v_{1} \ldots v_{k-2}$ denote a vertex of $T_{n, k}$.

Lemma 2.6.2. There is a directed path in $T_{n, k}$ from $v$ to any cyclic rotation of $v$.

Proof. Suppose we are interested in reaching the cyclic rotation $v^{\prime}=v_{m-1} v_{m} \ldots v_{k-3}$ $v_{k-2} v_{0} \ldots v_{m-2}$. We will prove that there exists a directed path from $v$ to $v^{\prime}$ in the transition digraph. Given a set $S$ and a subset $T$, let $\bar{T}=S \backslash T$. The following path certainly exists in $T_{n, k}$ :

$$
\begin{aligned}
v_{0} v_{1} \ldots v_{k-2} \longrightarrow v_{1} \ldots v_{k-2} b_{0} & \longrightarrow \\
& v_{2} \ldots v_{k-2} b_{0} b_{1} \longrightarrow \cdots \longrightarrow v_{m-1} \ldots v_{k-2} b_{0} \ldots b_{m-2}
\end{aligned}
$$

where $b_{j} \in \overline{\left\{v_{j}, v_{j+1}, \ldots, v_{k-2}, b_{0}, \ldots, b_{j-1}\right\}}$. The set $\left\{v_{j}, v_{j+1}, \ldots, v_{k-2}, b_{0}, \ldots, b_{j-1}\right\}$ contains $k-1$ elements, therefore, it is always possible to select an appropriate $b_{j}$ from $[n]$. Continuing the established path, the vertex $v_{m-1} \ldots v_{k-2} b_{0} \ldots b_{m-2}$ is adjacent to $v_{m} \ldots v_{k-2} b_{0} \ldots b_{m-2} b_{m-1}$. It is now possible to begin moving towards the permutation 
$v^{\prime}$. The value denoted by $v_{m-1}$ can be added to the right end of $v_{m} \ldots v_{k-2} b_{0} \ldots b_{m-2}$ $b_{m-1}$, to obtain a valid $k$-permutation, since $v_{m-1} \in \overline{\left\{v_{m}, \ldots, v_{k-2}, b_{0}, \ldots, b_{m-1}\right\}}$, hence, $v_{m} \ldots v_{k-2} b_{0} \ldots b_{m-2} b_{m-1}$ is adjacent to $v_{m+1} \ldots v_{k-2} b_{0} \ldots b_{m-2} b_{m-1} v_{m-1}$ in $T_{n, k}$. Continue the path by adding elements on the right in the order desired for the final permutation. The following path can be created:

$$
\begin{gathered}
v_{m+1} v_{m+2} \ldots v_{k-2} b_{0} \ldots b_{m-2} b_{m-1} v_{m-1} \rightarrow v_{m+2} \ldots v_{k-2} b_{0} \ldots b_{m-2} b_{m-1} v_{m-1} v_{m} \rightarrow \cdots \\
\ldots \rightarrow b_{0} b_{1} \ldots b_{m-1} v_{m-1} \ldots v_{k-2} \rightarrow b_{1} \ldots b_{m-1} v_{m-1} \ldots v_{k-2} v_{0} \rightarrow \cdots \\
\ldots \rightarrow v_{m-1} v_{m} \ldots v_{k-2} v_{0} \ldots v_{m-2}
\end{gathered}
$$

Thus, there exists a directed path from $v$ to any cyclic rotation of $v$.

Proof of Theorem 2.6.1. The existence of a Ucycle for $k$-permutations of $[n]$ is equivalent to the existence of an Euler cycle in the transition digraph, $T_{n, k}$. To prove an Euler cycle exists, we show that $T_{n, k}$ is strongly connected and that each vertex of $T_{n, k}$ has in-degree equal to out-degree (Theorem 2.5.1).

To show that $T_{n, k}$ is strongly connected, it is sufficient to show that $v$ is connected to $12 \ldots(k-1)$ and that $12 \ldots(k-1)$ is connected to $v$, for any vertex $v$. Let $v=v_{0} v_{1} \ldots v_{k-2}$ be a vertex of $T_{n, k}$. By connected we mean that there exists a directed path from the first vertex to the second. This is done in two steps:

(1) show $v$ is connected to some $w=w_{0} w_{1} \ldots w_{k-2}$, where $w_{0}<w_{1}<\ldots<w_{k-2}$, then 
(2) show $w$ is connected to $12 \ldots(k-1)$.

If $v_{0}<v_{1}<\ldots<v_{k-2}$, the first step is complete. Otherwise, $v_{m}<v_{m-1}$ for some $m \in\{1,2, \ldots, k-2\}$. Lemma 2.6.2 implies that there exists a directed path from $v_{0} v_{1} \ldots v_{m-1} v_{m} \ldots v_{k-2}$ to $v_{m-1} v_{m} \ldots v_{k-2} v_{0} \ldots v_{m-2}$, for any $m \in\{1,2, \ldots, k-1\}$. We now establish that there exists a directed path from $v_{m-1} v_{m} \ldots v_{m-2}$ to a vertex of the form $w$. Notice that because $n \geq k+1$ we can choose a pair of elements, say $x$ and $y$, from $\overline{\left\{v_{0}, v_{1}, \ldots, v_{k-2}\right\}}$. The vertex $v_{m-1} v_{m} \ldots v_{k-2} v_{0} \ldots v_{m-2}$ is adjacent to $v_{m} \ldots v_{k-2} v_{0} \ldots \ldots v_{m-2} x$, which is, in turn, adjacent to $v_{m+1} \ldots v_{k-2} v_{0} \ldots \ldots v_{m-2} x y$. Since there exists a directed path from a given vertex to each of its cyclic rotations, there exists a directed path from $v_{m+1} \ldots v_{k-2} v_{0} \ldots v_{m-2} x y$ to $x y v_{m+1} \ldots v_{k-2} v_{0} \ldots$ $v_{m-2}$. The vertex $x y v_{m+1} \ldots v_{k-2} v_{0} \ldots v_{m-2}$ is adjacent to $y v_{m+1} \ldots v_{k-2} v_{0} \ldots v_{m-2} v_{m}$ which, in turn, is adjacent to $v_{m+1} \ldots v_{k-2} v_{0} \ldots v_{m-2} v_{m} v_{m-1}$. Finally, this permutation is connected to its cyclic rotation $v_{0} v_{1} \ldots v_{m-2} v_{m} v_{m-1} v_{m+1} \ldots v_{k-2}$. The pair $v_{m}<v_{m-1}$ now appears in the desired order in the permutation. We continue to perform this procedure until we reach a vertex of the form $w=w_{0} w_{1} \ldots w_{k-2}$, where $w_{0}<w_{1}<\ldots<w_{k-2}$, to which $v$ is connected.

The second part of the proof involves showing that $w=w_{0} w_{1} \ldots w_{k-2}$, where $w_{0}<w_{1}<\ldots<w_{k-2}$, is connected to $12 \ldots(k-1)$. We give an explicit proof, adding a case omitted by Jackson. We must consider two possibilities for the value of $w_{0}$. If $w_{0}>1$, then $w_{i} \neq i+1$, for all $0 \leq i \leq k-2$, due to the strictly increasing 
order of elements in $w$. Therefore, $w_{0} w_{1} \ldots w_{k-2}$ is adjacent to $w_{1} \ldots w_{k-2} 1$, which is, in turn, adjacent to $w_{2} \ldots w_{k-2} 12$. By continuing to shift through $w$ in this manner we will reach $12 \ldots(k-1)$. If $w_{0}=1$, we must be careful in our approach. In this case, $w_{0} w_{1} \ldots w_{k-2}$ is not adjacent to $w_{1} \ldots w_{k-2} 1$ because such an edge would yield an invalid "permutation" starting and ending with 1 . Therefore, our first move is to a vertex $w_{1} \ldots w_{k-2} x, x \in \overline{\left\{w_{0}, w_{1}, \ldots, w_{k-2}\right\}}$, adjacent to $w$. The vertex $w_{1} \ldots w_{k-2} x$ is adjacent to $w_{2} \ldots w_{k-2} x 1$ since $w_{0}<w_{1}<\ldots<w_{k-2}$. The addition of $x$ means that if there exists another $w_{i}=i+1$, for some $1 \leq i \leq k-2$, there is no problem since the value $i+1$ will be added to the end of a permutation one step after it is removed from the front of a permutation. We may now proceed, as described above, to shift through $w$ until we reach $12 \ldots(k-1)$.

Jackson does not show that $12 \ldots(k-1)$ is connected to $v$. As this is not a trivial argument, we provide details here. To prove that $12 \ldots(k-1)$ is connected to $v$ we use the same tools as above, but different reasoning. Denote $12 \ldots(k-1)$ by $y_{0} y_{1} \ldots y_{k-2}$. If $v_{0} \notin\left\{y_{0}, y_{1}, \ldots, y_{k-2}\right\}$, then $y_{0} y_{1} \ldots y_{k-2}$ is adjacent to $y_{1} y_{2} \ldots y_{k-2} v_{0}$. Otherwise, $v_{0}=y_{i}$, for some $0 \leq i \leq k-2$ and, by cyclic rotation, $y_{0} y_{1} \ldots y_{k-2}$ is adjacent to $y_{i+1} \ldots y_{k-2} y_{0} \ldots y_{i}=y_{i+1} \ldots y_{k-2} y_{0} \ldots v_{0}$. Without loss of generality, assume we are in the second case. If $v_{1} \notin\left\{y_{0}, y_{1}, \ldots, y_{k-2}\right\}$, then $y_{i+1} \ldots y_{k-2} y_{0} \ldots v_{0}$ is adjacent to $y_{i+2} \ldots y_{k-2} y_{0} \ldots v_{0} v_{1}$. Otherwise, $v_{1}=y_{j}$, for some $0 \leq j \leq k-2, j \neq i$. Assume $j>i$. The vertex $y_{i+1} \ldots y_{j} \ldots y_{k-2} y_{0} \ldots v_{0}$ is adjacent to $y_{j} \ldots y_{k-2} y_{0} \ldots v_{0} \ldots y_{j-1}=$ 
$v_{1} \ldots y_{k-2} y_{0} \ldots v_{0} \ldots y_{j-1}$. There exists $x \in \overline{\left\{y_{0}, y_{1}, \ldots, y_{k-2}\right\}}$ such that $v_{1} y_{j+1} \ldots$ $\ldots y_{k-2} y_{0} \ldots v_{0} \ldots y_{j-1}$ is adjacent to $y_{j+1} \ldots y_{k-2} y_{0} \ldots v_{0} \ldots y_{j-1} x$. By cyclic rotation, this vertex is adjacent to $y_{i+1} \ldots y_{j-1} x y_{j+1} \ldots y_{k-2} y_{0} \ldots v_{0}$, which is, in turn, adjacent to $y_{i+2} \ldots y_{j-1} x y_{j+1} \ldots v_{0} v_{1}$. We continue in this manner until we reach vertex $v$. Therefore, we conclude that $T_{n, k}$ is strongly connected.

To see that the in-degree of each vertex is equal to its out-degree, consider the vertex $v=v_{0} v_{1} \ldots v_{k-2} \in V\left(T_{n, k}\right)$. This $(k-1)$-permutation can be extended to a $k$-permutation by adding one element that does not already appear in $v$. There are $n-(k-1)=n-k+1$ such elements. Let $v^{\prime}$ denote a vertex created by adding a new element to the beginning of $v$ and then dropping the element $v_{k-2}$. There is an edge from each $v^{\prime}$ to $v$ in $T_{n, k}$, therefore, the in-degree of $v$ is $n-k+1$. Similarly, let $v^{\prime \prime}$ denote a vertex created by adding a new element to the end of $v$ and then dropping the element $v_{0}$. There is an edge from $v$ to each $v^{\prime \prime}$ in $T_{n, k}$, therefore, the out-degree of $v$ is $n-k+1$. We have shown that both conditions of Theorem 2.5.1 are satisfied, therefore, $T_{n, k}$ is Eulerian.

We now turn to Ucycles for $n$-permutations of $[n]$, which we will simply refer to as permutations. There are at least three possible ways to define a Ucycle for permutations. The first was suggested by Chung et al. in [12]. A permutation of order $n, \pi=\pi_{1} \ldots \pi_{n}$, is order isomorphic to a sequence of length $n, s_{1} \ldots s_{n}$, if for $1 \leq i, j \leq n, \pi_{i}<\pi_{j}$ if and only if $s_{i}<s_{j}$. We will use the symbol $\sim$ to indicate that 
two permutations are order isomorphic. A Ucycle for permutations of $[n]$ is a circular sequence, $U=x_{0} \ldots x_{n !-1}$ of symbols from $\{1, \ldots, M\}$ in which every permutation is order isomorphic to some contiguous subsequence of length $n$. The aim is to find the smallest $M$ such that an order-isomorphic Ucycle exists. For $n>2, M$ must be at least $n+1$. It is conjectured by Chung et al. that there exist Ucycles for permutations of $[n]$ with $M=n+1$. The sequence 123415342154213541352435 is a Ucycle for permutations of [4] with $M=5$. The list of permutations represented by this sequence is shown in Table 11.

\begin{tabular}{|c|c|c|c|c|c|}
\hline Jcyc & & on & $\longleftrightarrow$ & & $n$ \\
\hline 234 & $\Longleftrightarrow$ & 1234 & 2135 & $\Longleftrightarrow$ & 2134 \\
\hline 2341 & $\Longleftrightarrow$ & 2341 & 1354 & $\Longleftrightarrow$ & 1243 \\
\hline 3415 & $\Longleftrightarrow$ & 2314 & 3541 & $\Longleftrightarrow$ & 2431 \\
\hline 1153 & $\Longleftrightarrow$ & 3142 & 5413 & $\Longleftrightarrow$ & 4312 \\
\hline 1534 & $\Longleftrightarrow$ & 1423 & 4135 & $\Longleftrightarrow$ & 3124 \\
\hline 5342 & $\Longleftrightarrow$ & 4231 & 1352 & $\Longleftrightarrow$ & 1342 \\
\hline 3421 & $\Longleftrightarrow$ & 3421 & 3524 & $\Longleftrightarrow$ & 2413 \\
\hline 4215 & $\Longleftrightarrow$ & 3214 & 5243 & $\Longleftrightarrow$ & 4132 \\
\hline 2154 & $\Longleftrightarrow$ & 2143 & 2435 & $\Longleftrightarrow$ & 1324 \\
\hline 1542 & $\Longleftrightarrow$ & 1432 & 4351 & $\Longleftrightarrow$ & 3241 \\
\hline 421 & $\Longleftrightarrow$ & 4321 & 3512 & $\Longleftrightarrow$ & 3412 \\
\hline 4213 & $\Longleftrightarrow$ & 4213 & 5123 & $\Longleftrightarrow$ & 4123 \\
\hline
\end{tabular}

Table 11: The representation of permutations of [4] given by the Ucycle 123415342154213541352435 with $M=5$ (read column-wise)

It is possible to construct a digraph for permutations of $[n]$ that is similar to a transition digraph; however, the graph provides less information than the transition 
digraph for $k$-permutations of $[n]$. Let $G_{n}$ denote the 'transition' digraph for permutations of $[n]$. The vertices are the permutations of $[n]$ and there is an edge from one vertex to another if the $(n-1)$-tail of the first is order-isomorphic to the $(n-1)$ head of the second. That is, there is an edge from $x_{0} x_{1} \ldots x_{n-1}$ to $y_{0} y_{1} \ldots y_{n-1}$ if $x_{1} \ldots x_{n-1}$ is order-isomorphic to $y_{0} \ldots y_{n-2}$. The following example illustrates this concept. Suppose that $n=3$ and that a portion of the Ucycle is . . $452 x \ldots$. We know $452 \sim 231$, however, there are several possibilities for the next block. If $x=1$, then $52 x \sim 321$ and hence there is an edge from 231 to 321 . If $x=3$ or 4 , then $52 x \sim 312$ and hence there is an edge from 231 to 312 . Finally, if $x=6$, then $52 x \sim 213$ and hence there is an edge from 231 to 213 . The 'transition' digraph for permutations of order 3 is shown in Figure 9.

The 'transition' digraph for permutations differs from transition digraphs we have seen to this point. The permutations are now represented by vertices rather than edges, therefore, we are interested in finding a Hamilton cycle in this digraph not an Euler cycle. A Hamilton cycle is a cycle that passes through each vertex of a graph exactly once. However, determining a Hamilton cycle in the 'transition' digraph does not automatically induce a Ucycle. It is necessary to translate the cycle into the Ucycle alphabet. Given a Hamilton cycle in the 'transition' digraph and an unknown Ucycle, $x_{0} x_{1} \ldots x_{n !-1}$, one can represent the implied inequalities between the elements $x_{0}, x_{1}, \ldots, x_{n !-1}$ by a partial order. An order-preserving mapping of 
$\left\{x_{0}, x_{1}, \ldots, x_{n !-1}\right\}$ onto $\{1,2, \ldots, M\}$ is required (implying the selection of a suitable $M)$. This procedure is referred to as lifting the Ucycle. Consider the Hamilton cycle $132,312,123,231,321,213$ in $G_{3}$ (see Figure 9). Suppose a Ucycle for the permutations of [3] is $x_{0} x_{1} x_{2} x_{3} x_{4} x_{5}$. We require a mapping $\phi$ from $\left\{x_{0}, \ldots, x_{5}\right\}$ onto $\{1,2, \ldots, M\}$ which preserves the partial order induced by the relations $x_{0} x_{1} x_{2} \sim 132$, $x_{1} x_{2} x_{3} \sim 312$, and so on. Choose $M=4$ and let $\phi\left(x_{0}\right)=1, \phi\left(x_{2}\right)=\phi\left(x_{5}\right)=2$, $\phi\left(x_{3}\right)=3$, and $\phi\left(x_{1}\right)=\phi\left(x_{4}\right)=4$. This gives the Ucycle 142342 for the permutations of [3].

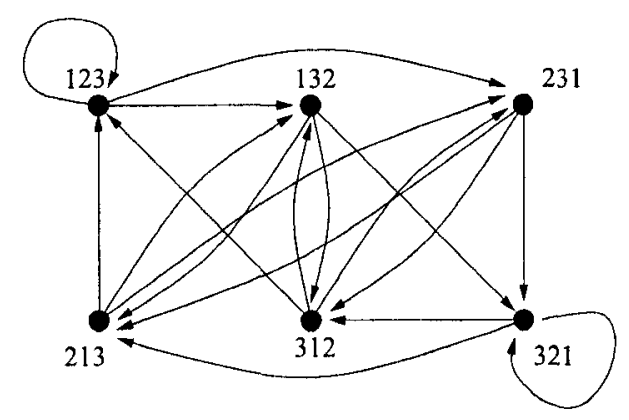

Figure 9: The 'transition' digraph for permutations of order 3

Hurlbert has proved that order-isomorphic Ucycles for permutations exist for all $n$ by constructing such cycles [38]. Let $U$ be a Ucycle for permutations of $[n]$. Define the height of $U$, denoted $h(U)$, to be the number of distinct symbols used in $U$. Let $h(n)$ denote the minimum height required over all possible Ucycles for permutations of $[n]$. The conjecture of Chung et al. is equivalent to $h(n)=n+1$, for all $n \in \mathbb{N}$. Hurlbert has been able to show that $h(n) \leq\lceil 5 n / 2\rceil-4$, for all $n \in \mathbb{N}$ [38].

We now turn to a second idea of equivalence for permutations. Let $1_{m}$ denote 
the vector of $m$ ones. Let $\Pi_{i, j}^{k}$ denote the set of all $k$-permutations of $\{i, i+1, \ldots, j\}$ and write elements of $\Pi_{i, j}^{k}$ as length $k$ vectors of distinct terms from $\{i, i+1, \ldots, j\}$. Define the following equivalence relation on $\Pi_{0, n}^{m}$. For $\mathbf{a}, \mathbf{b} \in \Pi_{0, n}^{m}, \mathbf{a} \sim \mathbf{b}$ if and only if $\mathbf{a}-\mathbf{b} \equiv k \cdot 1_{m}(\bmod n+1)$ for some $k \in \mathbb{N}$. This $\sim$ is an equivalence relation and there are $n ! /(n-m)$ ! equivalence classes of the elements of $\Pi_{0, n}^{m}$, each of which corresponds to an element of $\Pi_{1, n}^{m}$. This means we have a representation of permutations of $[n]$ that can be viewed as equivalence classes of $n$-permutations of $[n+1]$. Define an equivalence class Ucycle to be a sequence of length $n$ ! such that among the length- $n$ subsequences each equivalence class is represented exactly once. Hurlbert and Issak have shown that, given this equivalence relation, there exist equivalence class Ucycles representing $\Pi_{1, n}^{n}[37]$. These equivalence class Ucycles use $n+1$ symbols to represent Ucycles for permutations of $[n]$.

Theorem 2.6.3. ([37]) There exists a complete family of equivalence class Ucycles for permutations of $\{1,2, \ldots, n\}$ using the symbols $\{0,1, \ldots, n\}$.

A third way to define a Ucycle for permutations requires that the original alphabet be maintained, however, it relaxes the condition that each permutation of order $n$ appear exactly once. Using this definition, the goal is to find the shortest circular sequence of symbols from $[n]$ such that every permutation of $[n]$ appears as a contiguous subsequence at least once. 


\subsection{2 $k$-subsets of $n$-sets}

The largest body of work on Ucycles is focused on determining the existence of Ucycles for $k$-subsets of $n$-sets. This is due, in part, to the fact that it has thus far proved to be a much more difficult question to answer than the existence of Ucycles for $k$ permutations and partitions. Throughout this section, we assume $1<k<n$, as the two extreme cases are trivial. As a result, the alphabet used in a Ucycle for $k$-subsets of $[n]$ is exactly the $n$-set. It seems this would make the problem easier to handle; however, we will see that the existence of Ucycles for $k$-subsets of $n$-sets is known only for specific values of $k$.

Because we are dealing with subsets, once a given subset has been represented in the Ucycle it may not appear in any other order elsewhere in the sequence. This means that determining a transition digraph is difficult as the vertices and edges are dependent on the part of the Ucycle that has already been fixed. For example, if the subset $\{1,2,3\}$ is represented in the Ucycle as 123 then the arc $\{1,2,3\} \rightarrow$ $\{2,3,4\}$ exists in the transition digraph. However, if the subset is represented as $213,\{1,2,3\} \rightarrow\{2,3,4\}$ cannot be an arc in the transition digraph. The following modular condition is easily established.

Lemma 2.6.4. ([12]) If there is a Ucycle for k-subsets of $[n]$, then $k$ divides $\left(\begin{array}{c}n-1 \\ k-1\end{array}\right)$.

Ucycles exist for $k=2$ as long as the condition of Lemma 2.6.4 is satisfied, that is, as long as $n$ is odd. The following two theorems are due to Jackson. 
Theorem 2.6.5. ([39]) There exist Ucycles for 3 -subsets of $[n]$, when $n \geq 8$, if and only if $\left(\begin{array}{c}n-1 \\ 2\end{array}\right) \equiv 0(\bmod 3)$.

Before presenting a proof of Theorem 2.6.5, we introduce some helpful machinery. Let $X=\left\{x_{1} x_{2} \ldots x_{k}\right\}$ be a $k$-subset of $[n]$ written such that $x_{1}<x_{2}<\ldots<x_{k}$. To visualize the following idea, think of the integers from zero to $v$ arranged around a circle. Two $k$-subsets are of the same type if they differ by just a rotation around the circle. For example, 123 is of the same type as 345 . Formally we can define the type of $X$ to be $\left(x_{2}-x_{1}, x_{3}-x_{2}, \ldots, x_{k}-x_{k-1}, x_{1}-x_{k}\right)$, with arithmetic performed modulo $n$. An ordered $k$-subset can be uniquely identified by its first entry and (the first $k-1$ entries of $)$ its type. The set of all $k$-subsets of $[n]$ of a certain type is given by omitting the largest difference that is unequal to any of the other $k-1$ differences. Proof of Theorem 2.6.5. To prove that Ucycles for 3-subsets of $[n]$ exist, a similar argument to that involved in proving the existence of Ucycles for permutations is employed. A subsequence of $k$ elements in a Ucycle with differences $d_{1} d_{2} \ldots d_{k-1}$ (we have dropped the brackets and commas between differences and we ignore the difference between the last and the first elements) must be followed by a subsequence of $k$ elements with differences $d_{2} \ldots d_{k-1} d_{k}$. A transition digraph can be created for these difference types with vertices representing vectors of length $k-2$ and each edge representing a length $k-1$ difference type (which in turn represents many possible $k$-subsets). Given vertices $d_{1} \ldots d_{k-2}$ and $d_{2} \ldots d_{k-1}$, there is a directed edge between 
them representing the difference type $d_{1} \ldots d_{k-1}$.

For 3-subsets, the difference types are of the form (1) three unequal differences, or (2) two equal differences and a third number different from these two (but never three equal differences as $\operatorname{gcd}(n, 3)=1$ ). Since we can drop one of the differences without any loss of information, the types are written as (1) $i j$ or $j i$, where $i \neq j$, or (2) ii. The transition digraph for 3-subsets of [8] is pictured in Figure 10. The possible difference types modulo 8 are also listed, with a space separating the two differences used to represent each type (we always drop the largest difference that appears exactly once).

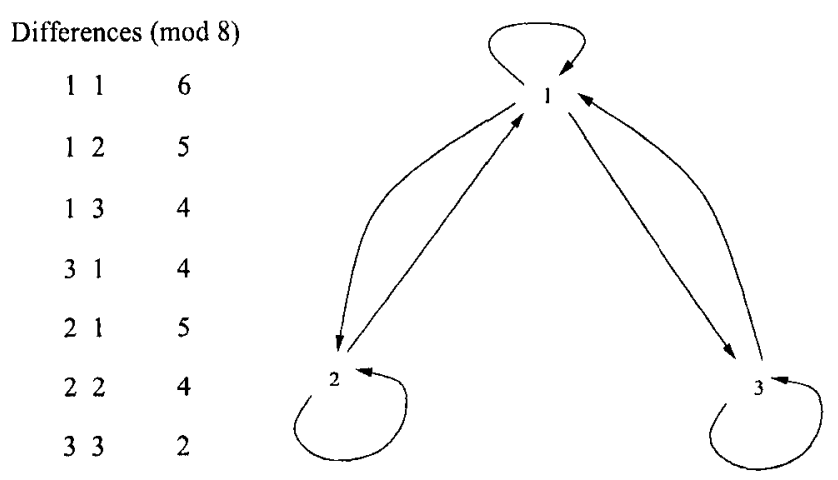

Figure 10: The transition digraph for 3-subsets of [8]

For $n=8$, the approach to finding a Ucycle for 3-subsets of [8] reduces to the following. An Euler cycle in the transition digraph defines a circular arrangement that contains each difference type uniquely (as a 2 -set). The sum of the differences in the circular arrangement is coprime to $n$, therefore, a list which contains each 3-subset of [8] exactly once can be obtained by starting with a given initial value 
in [8] and successively adding this list of differences $n$ times before returning to the starting point. Figure 11 is a Ucycle for 3-subsets of an 8-set obtained by taking the difference list 11221331 (an Euler cycle in Figure 10) and successively adding this list of differences with starting point 1.

When $n>8$ a little more work is required. Recall that 3-subsets can be represented by their first element and two differences, thus $\left(x, d_{1} d_{2}\right)$ represents the subset $\left\{x, x+d_{1}, x+d_{1}+d_{2}\right\}$. In a Ucycle, the 3 -subset represented by $\left(x, d_{1} d_{2}\right)$ is followed by the 3 -subset represented by $\left(x+d_{1}, d_{2} d_{3}\right)$. Define an expanded transition digraph with vertices $(x, d)$ such that the edge from vertex $\left(x, d_{1}\right)$ to $\left(x+d_{1}, d_{2}\right)$ is the 3 -subset representative $\left(x, d_{1} d_{2}\right)$. Each vertex has the same in-degree and out-degree and one can show that the graph is also strongly connected, therefore, appealing to Theorem 2.5.1, there is an Euler cycle in the expanded transition digraph.

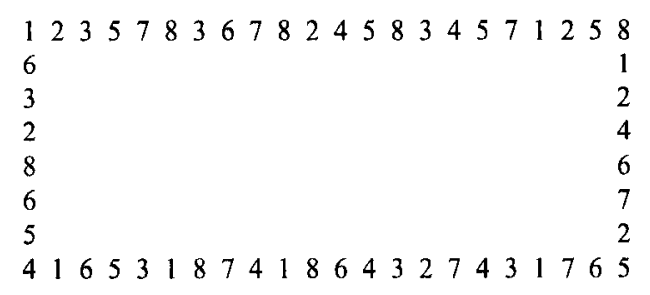

Figure 11: A Ucycle for 3-subsets of [8]

Using a similar approach to that used in proving Theorem 2.6.5, Jackson has also partially determined the existence of Ucycles for 4-subsets.

Theorem 2.6.6. ([39]) There exist Ucycles for 4-subsets of $[n], n \geq 9$, provided $\left(\begin{array}{c}n-1 \\ 3\end{array}\right) \equiv 0(\bmod 4)$ and $\operatorname{gcd}(n, 4)=1$. 
Note that there are values of $n$, namely $n \equiv 2(\bmod 8)$, that satisfy the modular condition of Lemma 2.6.4, but are not covered by Theorem 2.6.6. These cases where $n$ is not coprime to four remain unresolved.

Using much the same approach as Jackson, Hurlbert has also shown that Ucycles exist for 3-subsets of $[n]$ and 4 -subsets of $[n]$, when $n$ and $k$ are coprime [35]. In addition, he has proved the existence of Ucycles for 6-subsets of $n$ when $\operatorname{gcd}(n, 6)=1$ and $n$ is sufficiently large. Nothing is known about the existence of Ucycles for $k$-subsets of $[n]$ for $k=5$ or for $k \geq 7$, although computer searches have constructed some specific examples of Ucycles for higher $k$. Chung et al. have made the following conjecture, suggesting that the statement of Lemma 2.6 .4 is both necessary and sufficient.

Conjecture 2.6.7. ([12]) There exist Ucycles for $k$-subsets of [ $n]$, provided $k$ divides $\left(\begin{array}{c}n-1 \\ k-1\end{array}\right)$ and $n$ is sufficiently large.

However, Stevens et al. have shown that Ucycles do not exist for $(n-2)$-subsets of $[n]$, when $n \geq 4$ [53]. That is, $n$ must be at least $k+3$ for a Ucycle representing the $k$-subsets of $[n]$ to exist. The approach in [53] is to determine structural features that these Ucycles would have if they existed, and then show that these features cannot possibly be realized.

As with permutations, the non-existence of a Ucycle for $k$-subsets of $[n]$ does not end the investigation. It is possible to search for "how close" one can get to such a Ucycle in two ways. One way is to find the longest sequence having all properties 
of a Ucycle, except not every subset will appear; this is called a cyclic packing word. The definition can be further relaxed to a non-cyclic sequence having Ucycle properties containing as many $k$-subsets of $[n]$ as possible. Such a sequence is called a linear packing word. The other way to get "close" to a Ucycle is to find the shortest sequence such that every subset appears at least once. Such a sequence is called a cyclic covering word. These are similar notions to packings and coverings in design theory.

Theorem 2.6.8. ([53]) The longest possible cyclic packing word of $(n-2)$-subsets of $[n]$ has length $n$ and a word achieving this bound always exists: $12 \cdots(n-1) n$.

Theorem 2.6.9. ([53]) The longest possible linear packing word of $(n-2)$-subsets of $[n]$ has length $3 n-6$ and a word achieving this bound always exists:

$$
\begin{aligned}
& 123 \cdots(n-4)(n-3)(n-2)(n-1) 123 \cdots \\
& \cdots(n-4)(n-3) n(n-1) 123 \cdots(n-5)(n-4)
\end{aligned}
$$

Hurlbert extended the definition of Ucycles for $k$-subsets of $[n]$ to consider sequences in which every $k$-subset appears as a consecutive subsequence at least once and all $k$-subsets appear equally often. These are called $t$-cover Ucycles, where $t$ is the number of times each subset appears. Naturally, these will be cyclic sequences of length $t \cdot\left(\begin{array}{l}n \\ k\end{array}\right)$. The following lemma is an extension of Lemma 2.6.4 to multicover Ucycles. 
Lemma 2.6.10. ([36]) If there is a $t$-cover Ucycle for $k$-subsets of $[n]$, then $k$ divides $t \cdot\left(\begin{array}{c}n-1 \\ k-1\end{array}\right)$.

Hurlbert's approach is to find the smallest $t$, given $n$ and $k$, such that a $t$-cover Ucycle exists. Denote this value by $U(n, k)$. Note that the known results for the existence of Ucycles for $k$-subsets of $n$-sets imply that for $k=2,3,4,6, n$ coprime to $k$ and $n$ sufficiently large, $U(n, k)=1$.

Theorem 2.6.11. ([36]) $U(n, k) \leq k$ for all $n \geq k$.

We have found that the corollary to this theorem given by Hurlbert in [38] is false. However, the following corollary - a modified version of that given by Hurlbert is true.

Corollary 2.6.12. If $k$ is a prime power and $k$ divides $n$, then $U(n, k)=k$.

The proof relies on the following lemma due to E. Kummer stated in [38]. Given a prime $p$ and an integer $x$, let $v_{p}(x)$ denote the maximum exponent $r$ such that $p^{r}$ divides $x$.

Lemma 2.6.13. (See [38]) $v_{p}\left(\left(\begin{array}{l}n \\ k\end{array}\right)\right)$ is equal to the number of borrows needed when subtracting $k$ from $n$ in base $p$.

Proof of Corollary 2.6.12. Suppose $k=p^{q}$, where $p$ is prime, and suppose $n=m k$, $m \in \mathbb{N}$. We wish to determine the divisibility of $\left(\begin{array}{c}n-1 \\ k-1\end{array}\right)$ by $p$. Written in base $p$

$$
k=1 \underbrace{00 \ldots 0}_{q} \text { and } n=x_{1} \ldots x_{i} \underbrace{0 \ldots 0}_{q} \text {. }
$$


So,

$$
k-1=\underbrace{(p-1)(p-1) \ldots(p-1)}_{q} \text { and } n-1=y_{1} \ldots y_{i} \underbrace{(p-1) \ldots(p-1)}_{q} .
$$

Therefore,

$$
(n-1)-(k-1)=y_{1} \ldots y_{i} \underbrace{0 \ldots 0}_{q},
$$

and no borrows are required. Lemma 2.6 .13 implies that zero is the largest exponent of $p$ that divides $\left(\begin{array}{c}n-1 \\ k-1\end{array}\right)$. Lemma 2.6 .10 requires that $k=p^{q}$ divide $U(n, k) \cdot\left(\begin{array}{c}n-1 \\ k-1\end{array}\right)$, so $k$ must divide $U(n, k)$. Therefore, since $U(n, k) \leq k, U(n, k)=k$.

\subsubsection{Partitions of an $n$-set}

Partitions of an $n$-set also require some adjustment to the definition of a Ucycle. The number of partitions of $[n]$ is the sum of the Stirling numbers of the second kind for $n$ and all $k$ between 1 and $n$. To represent a partition of $[n]$ we use a string of length $n$ with elements from $A=\{a, b, c, \ldots\}$. We put $i, j \in[n]$ in the same class of the partition if and only if the $i^{\text {th }}$ and $j^{\text {th }}$ entries of the string are the same. For example, abacbcdc represents the partition $13|25| 468 \mid 7$. Thus, abcbccccddcdeec is a Ucycle for the partitions of [4]. Create a 'transition' digraph for the partitions of $[n]$ with vertices representing the partitions, and place a directed edge from vertex $u$ to vertex $v$ if the alphabetic sequence representing partition $u$ can be followed (in a Ucycle) by the alphabetic sequence representing partition $v$. Figure 12 illustrates the 'transition' digraph for partitions of [3]. 


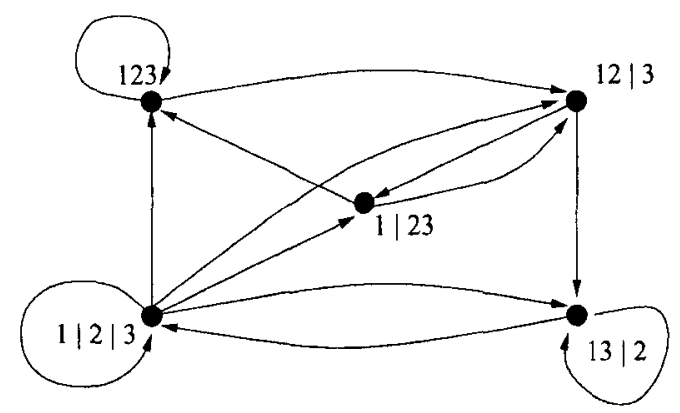

Figure 12: The 'transition' digraph for partitions of [3]

In order to find a Ucycle for the partitions of $[n]$ we must find a Hamilton cycle in the 'transition' digraph. As for $k$-subsets of $[n]$, finding such a Hamilton cycle does not guarantee the existence of a Ucycle as it is necessary to "lift" the Hamilton cycle to a Ucycle by assigning appropriate symbols in order to realize the corresponding partitions. There is a Hamilton cycle in the digraph shown in Figure 12, but it is not possible to lift it to a Ucycle. Suppose the Ucycle for partitions of [3] is $x_{0} x_{1} x_{2} x_{3} x_{4}$. Given the Hamilton cycle $123,12|3,13| 2,1|2| 3,1 \mid 23$, we find that $x_{0}=x_{1}=x_{2}$, $x_{3} \neq x_{2}, x_{4}=x_{2}$ and $x_{0} \neq x_{4}$, which is a contradiction. In fact, there is no Ucycle for the partitions of $\{1,2,3\}$ [12]. However, Chung, et al. claim (but do not explicitly prove) that it is possible to lift Hamilton cycles for $n \geq 4$ [12]. The problem with Hamilton cycles for $n=3$ (and a potential problem in general) is that the inequalities implied by the lifting of the cycle result in $x \neq x$, for some $x$ in the Ucycle. However, it is possible to prevent such an occurrence when $n \geq 4$ by requiring that a certain sequence of partitions occur in the cycle and that this sequence not contain the partition of $[n]$ into $n$ parts. 
Theorem 2.6.14. ([12], [38]) There exist Ucycles for partitions of $[n]$ when $n \geq 4$.

Proof. We show the validity of this statement for partitions of $n \geq 8$ by exhibiting the necessary subsequence of partitions that ensures the avoidance of the $x \neq x$ condition:

(A) For $n$ even, the sequence is $a b \ldots b a \ldots a b a c \ldots c d e d \ldots$ de $\ldots$ ede, and

(B) for $n$ odd, the sequence is $a b \ldots b a b \ldots b a b c \ldots c d e d \ldots$ ded $\ldots$ ded,

where $y \ldots y$ indicates a contiguous sequence of the letter $y$. The large blocks of the letters $a, b, d$ and $e$ have length $\lfloor(n-4) / 2\rfloor$ and the center block of the letter $c$ has length $n$. Note that this subsequence of the letter $c$ represents the partition of $[n]$ into one group. Suppose the Ucycle looks like $x_{0} x_{1} \ldots x_{m-1}$ with $x_{i}$ to the left of the center block and $x_{j}$ to the right of the center block. Because the center block is of length $n$ there is no relation implied between $x_{i}$ and $x_{j}$. The removal of the edges represented by sequence $A$ or sequence $B$ from the 'transition' digraph does not disconnect it. It is possible to find a Hamilton cycle in the rest of the digraph and adjoin it to either sequence $A$ or $B$ in order to produce a Ucycle.

One can also consider $k$-partitions of $[n]$, that is, partitions of $[n]$ into $k$ classes, where $2 \leq k \leq n-1$. Little is known about these cases. Hurlbert has shown that Ucycles for $k$-partitions of $[n]$ exist for $n \geq 3$ odd and $k=n-1$ [38]. Casteels has shown that if $n$ is even, there do not exist Ucycles for $(n-1)$-partitions of $[n]$, and 
furthermore, if $n$ is odd, there is an algebraic way to enumerate all Ucycles of this form [10]. 


\section{Chapter 3}

\section{Ordering the blocks of designs}

Just as there are many ways to order $k$-subsets, partitions and permutations, there are many ways to order the blocks of a design. A listing of the blocks of a design such that there is a minimal change between consecutive blocks is a combinatorial Gray code. Note that the minimal change definition will depend on the design parameters. For example, the blocks of a TTS $(v)$ can theoretically be listed such that consecutive blocks differ in exactly one element. On the other hand, for a design with $\lambda=1$, the strongest minimal change listing would have exactly one element remaining the same from one block to the next. In the previous chapter, we saw that minimal change can also be defined structurally. Ucycles are sequences that adhere to such a definition, although the contents of consecutive objects may also fit the Gray code concept of minimal change. Another rule for ordering blocks of designs that relates 
both to content and structure of blocks employs configurations. Unlike Gray codes and Ucycles, configuration ordering is an ordering paradigm unique to designs.

In this chapter, we define Gray codes and Ucycles for block designs. In the second half of the chapter, we look at configuration orderings. We start with Cohen and Colbourn's definition of configuration ordering and review some of their results in this area. This will illustrate that configuration ordering is a natural idea with practical applications. Then, we introduce a generalization of this definition and present some previously known results which can be expressed in terms of configuration ordering. We will see that generalized configuration orderings provide a different language with which to discuss Gray codes and Ucycles for designs.

\subsection{Gray Codes for Block Designs}

A Gray code for a $(v, k, \lambda)$-BIBD is similar to a Gray code for $k$-subsets of an $n$-set. In this dissertation, we deal only with designs for which the elements in a block are not considered to be ordered, therefore, we need only consider the most basic definition of a Gray code for $k$-subsets of an $n$-set. A list containing all $k$-subsets of an $n$-set is said to be in minimal change order if consecutive subsets differ by the deletion of one element and the insertion of another. Such an ordering is not always possible for the blocks of designs. The definition of minimal change for Gray codes for block designs is dependent on the size of the blocks in question and the strength and index of the 
design. For example, the blocks of a $(v, k, 1)$-BIBD intersect in at most one point, therefore, a Gray code for these blocks would have consecutive blocks intersecting in exactly one point regardless of the value of $k$.

Definition 3.1.1. ( $\kappa$-intersecting Gray code) $A \kappa$-intersecting Gray code for $a(v, k, \lambda)-B I B D$ is a listing of the blocks of the design such that consecutive blocks intersect in exactly $\kappa$ points.

When the required minimal change property holds between the first and last blocks of a Gray code, we refer to it as a Gray cycle. The intersection value $(\kappa)$ of a Gray code is related to the strength, block size, and index of the design in question. For example, a TTS $(v)$ may admit a 2-intersecting Gray code for its blocks because every pair of points appears in two blocks. However, an $\operatorname{STS}(v)$ cannot possibly admit a 2-intersecting Gray code, since every pair of points appears in a single block, but it may admit a 1-intersecting Gray code.

We are not aware of any results directly regarding Gray codes for the blocks of designs. The revolving door algorithm (described in Section 2.4) implies the existence of a 1-intersecting Gray cycle for each $(v, 2,1)$-BIBD, as these designs are exactly the collection of all 2-subsets of a $v$-set. The existence of a Hamilton cycle in the 1-block intersection graph of all $(v, k, 1)$-BIBDs ([33]) implies the existence of 1-intersecting Gray cycles for the blocks of these designs. In general, the existence of $\kappa$-intersecting 
Gray cycles for $(v, k, \lambda)$-BIBDs, where $1 \leq \kappa \leq k-1$, can be established by determining if the $\kappa$-block intersection graph of the BIBD is Hamiltonian. On the other hand, a Ucycle of rank $k$ for the blocks of a $(v, k, \lambda)$-BIBD is also a $(k-1)$-intersecting Gray cycle for the design. We define Ucycles for block designs in the next section.

\subsection{Universal Cycles for Block Designs}

As evidenced by the discussion in Section 2.6 , it is first necessary to fix a formal definition of a Ucycle for a block design. In particular, what (size of) alphabet will be used in the Ucycle and how will blocks be represented by this alphabet? One possible representation of a Ucycle for a $(v, k, \lambda)$-BIBD is a sequence of elements such that every length $k$ subsequence is a unique block of the design and such that every block of the design is represented exactly once. We re-phrase this definition in the formal language of Chung, et al. Let $(V, \mathcal{B})$ be a $(v, k, \lambda)$-BIBD. Let $b=|\mathcal{B}|$ and assume each $B \in \mathcal{B}$ is specified by a sequence $\left\langle x_{0}, x_{1}, \ldots, x_{k-1}\right\rangle$, where, for $0 \leq i \leq k-1, x_{i} \in B$. The sequence $\mathbf{U}=a_{0}, a_{1}, \ldots, a_{b-1}$ is a Ucycle for $(V, \mathcal{B})$ if $\left\langle a_{i}, a_{i+1}, \ldots, a_{i+k-1}\right\rangle, 0 \leq i \leq b-1$, runs through each element of $\mathcal{B}$ exactly once, where index addition is performed modulo $b$. Ucycles of this type exist for all $(v, 2,1)$ BIBDs with odd $v$. This is proved by Jackson in [39], although he refers to the collection of blocks as the set of 2-combinations of $[v]$. However, in general, there are three problems with this definition. First, we have restricted ourselves to balanced 
incomplete block designs and there is no obvious way to extend the definition to include pairwise balanced designs. Second, the definition fails to work for many BIBDs because it requires $\lambda \geq k-1$. Third, we will run into trouble with higher strength designs. Given a $t-(v, k, \lambda)$ design and a sequence of points from this design, every set of $t$ consecutive points will appear in $k-t+1$ blocks. As a result, we must have $\lambda \geq k-t+1$. In fact, every set of $s$ consecutive points, for $1 \leq s \leq t$, will appear in $k-s+1$ blocks. So we must have $\lambda_{s} \geq k-s+1$, for all $1 \leq s \leq t$, where

$$
\lambda_{s}=\lambda_{t} \frac{\left(\begin{array}{c}
v-s \\
t-s
\end{array}\right)}{\left(\begin{array}{c}
k-s \\
t-s
\end{array}\right)}
$$

and $\lambda_{t}=\lambda$.

Consider the Fano plane pictured in Figure 3 (page 18). Any pair of points defines a unique block of this design, hence starting the Ucycle with the sequence 013 means we cannot add another element to the sequence as no block other than $\{0,1,3\}$ contains 1 and 3. One resolution is to define a Ucycle for the Fano plane (or any other $(v, k, 1)$-BIBD, or $(v, K, 1)$-PBD) as a list of elements from $V$ such that every length two subsequence represents a unique block and such that each block is represented exactly once. Under this definition, a Ucycle for the Fano plane in Figure 3 is 3150642. This represents the blocks in the order: $\{0,1,3\},\{1,5,6\},\{0,4,5\},\{0,2,6\},\{3,4,6\}$, $\{1,2,4\},\{2,3,5\}$. On the other hand, the pairwise balanced design obtained by deleting a single point (for example, the point 6 in Figure 3) from the Fano plane does not have a Ucycle under this definition. Thus, we consider this to be a non-trivial 
definition of a Ucycle for a PBD. This is a natural representation for objects in a Ucycle and similar representations have been used for other combinatorial objects. For example, Jackson and Buhler have looked at Ucycles for all subspaces of dimension $x, x \geq 2$, in a vector space of dimension greater than $x$. For their representation of a subspace they use pairs of vectors from a basis of the subspace [8].

A single definition covers both types of Ucycles for PBDs discussed above.

Definition 3.2.1. (Ucycle of $\operatorname{Rank} \kappa)$ Let $(V, \mathcal{B})$ be $a(v, K, \lambda)-P B D$. Let $b=|\mathcal{B}|$ and let each $B \in \mathcal{B}$, be represented by the sequence $\left\langle x_{0}, x_{1}, \ldots, x_{\kappa-1}\right\rangle$, where, for $0 \leq i \leq \kappa-1, x_{i} \in B$. The sequence $\mathbf{U}=a_{0}, a_{1}, \ldots, a_{b-1}$ is a Ucycle of rank $\kappa$ for $(V, \mathcal{B})$ if $\left\langle a_{i}, a_{i+1}, a_{i+2}, \ldots a_{i+\kappa-1}\right\rangle, 0 \leq i \leq b-1$, runs through the representation of each element of $\mathcal{B}$ exactly once, where index addition is performed modulo $b$.

The key point here is that blocks can be represented by objects of various ranks, although all blocks within a given Ucycle must have representations of the same rank. The obvious choices for rank are strength and block size. For example, when representing the whole block of a TTS $(v)$ we would use an object of rank three. However, it is not always possible to use $k$-subsets to represent blocks of size $k$. For example, a $2-(v, k, \lambda)$ design with $\lambda<k-1$ does not admit a Ucycle where the representative of each block is the block itself. In this case, we represent a block by a pair of points appearing in that block, that is by an object of rank two which is the strength of the design. Before stating explicit definitions for rank two and rank three 
Ucycles, we state a necessary condition for the existence of a rank $\kappa$ Ucycle. Recall that $r_{x}$ is the number of blocks in which the point $x$ appears.

Lemma 3.2.1. If there exists a rank $\kappa$ Ucycle, $U$, for the design $S=(V, \mathcal{B})$, then each $x \in V$ appears at most $\left\lfloor r_{x} / \kappa\right\rfloor$ times in $U$ which implies $\sum_{x \in V}\left\lfloor r_{x} / \kappa\right\rfloor \geq b$.

Proof. Suppose $U=a_{0}, a_{1}, \ldots, a_{b-1}$ is a Ucycle of rank $\kappa$ representing the blocks of $S$. Consider a fixed symbol $a_{i}=x$ in $U$. The symbols $a_{i+j}$, for $-\kappa<j<\kappa$, must not be equal to $x$, therefore, each copy of $x$ in $U$ occurs in exactly $\kappa$ sets of $\kappa$ consecutive points in $U$. Each of these $\kappa$ consecutive point sets represents a block of the design containing the point $x$. Let $n_{x}$ represent the number of times $x$ appears in $U$. The point $x$ appears in exactly $r_{x}$ blocks of the design, however, a block containing $x$ may not be represented in $U$ by a set including $x$. Therefore, $n_{x} \leq\left\lfloor r_{x} / \kappa\right\rfloor$. Since $U$ is of length $b, \sum_{x \in V} n_{x}=b$ and we have $\sum_{x \in V}\left\lfloor r_{x} / \kappa\right\rfloor \geq b$.

Lemma 3.2.1 can be generalized as follows. Let $\mathcal{S}_{s}^{v}$ represent the collection of $s$-subsets of a $v$-set. For a given design $S=(V, \mathcal{B})$, let $A$ be a subset of $V$ and let $r_{A}$ denote the number of blocks of the design that the subset $A$ appears in. If $s \leq t$, where $t$ is the strength of $S$, an $s$-set $A$ appears in exactly

$$
r_{A}=\frac{\lambda_{t}\left(\begin{array}{c}
v-s \\
t-s
\end{array}\right)}{\left(\begin{array}{c}
k-s \\
t-s
\end{array}\right)}
$$

blocks of the design. 
Lemma 3.2.2. If there exists a rank $\kappa$ Ucycle for the design $S=(V, \mathcal{B})$, then

$$
\sum_{A \in \mathcal{S}_{s}^{v}}\left\lfloor\frac{r_{A}}{(\kappa-s+1)}\right\rfloor \geq b
$$

where $v=|V|$ and $s=|A|$.

Proof. The proof proceeds exactly as in the proof of Lemma 3.2.1 with $n_{A}$ representing the number of times a subset $A$ appears consecutively in $U$ (regardless of order). Each contiguous set of $s$ elements in $U$ appears in $\kappa-s+1$ blocks, however, a block containing $A$ may not be represented in $U$ by a $\kappa$-set containing $A$ as a contiguous subsequence of $s$ elements. Therefore, $n_{A} \leq\left\lfloor r_{A} /(\kappa-s+1)\right\rfloor$. Since $U$ is of length $b$, $\sum_{A \in \mathcal{S}_{s}^{v}} n_{A}=b$ and we have $\sum_{A \in \mathcal{S}_{s}^{v}}\left\lfloor r_{A} /(\kappa-s+1)\right\rfloor \geq b$.

Although Lemmas 3.2.1 and 3.2.2 are general necessary conditions for the existence of a rank $\kappa$ Ucycle, these conditions are weak because of the allowance for variable rank. If the design is regular (that is, has all blocks the same size) and the rank of the Ucycle is equal to the size of the blocks of the design, we have the following refinement of Lemma 3.2.1.

Lemma 3.2.3. Let $S=(V, \mathcal{B})$ be $a(v, k, \lambda)$-BIBD. If there exists a rank $k$ Ucycle for $S$, then $k$ divides $r$, where $r=\lambda(v-1) /(k-1)$.

Proof. Let $U$ be a rank $k$ Ucycle for $S$. The rank of $U$ is equal to the block size of $S$, hence, each block represents itself in $U$. Consider a fixed symbol $a_{i}$ in $U$ and suppose $a_{i}=x$. The symbols $a_{i+j}$, for $-k<j<k$, must not be equal to $x$, therefore, each 
copy of $x$ in $U$ occurs in exactly $k k$-subsequences of consecutive points in $U$. Each $k$-subsequence represents a block of $S$ containing $x$; there are $r=\lambda(v-1) /(k-1)$ such blocks. Therefore, $x$ must appear $r / k$ times in $U$. As this value must be an integer, we must have $k \mid r$.

In this dissertation, the only Ucycles with rank equal to block size that we consider are for triple systems, therefore, we restrict the definition of a rank three Ucycle to the following.

Definition 3.2.2. (Ucycle of rank three) Let $(V, \mathcal{B})$ be a $(v, 3, \lambda)-B I B D$, with $\lambda \geq 2$. Let $b=|\mathcal{B}|$ and let each block $B \in \mathcal{B}$ be represented by the sequence $\left\langle x_{0}, x_{1}, x_{2}\right\rangle$, where $x_{i} \in B$ for $0 \leq i \leq 2$. The sequence $\mathbf{U}=a_{0}, a_{1}, \ldots, a_{b-1}$ is a Ucycle for the $(v, 3, \lambda)-B I B D$ if $\left\langle a_{i}, a_{i+1}, a_{i+2}\right\rangle, 0 \leq i \leq b-1$, runs through each element of $\mathcal{B}$ exactly once, where index addition is performed modulo $b$.

Figure 13 represents a Ucycle of rank three for a TTS(7). In Section 4.1, we prove that for $v \equiv 1,4,7(\bmod 12)$, with sporadic exceptions, there exists a TTS $(v)$ which has a rank three Ucycle representation.

For triple systems with $\lambda=1$, and for all other PBDs, we will look at the existence of rank two Ucycles. In these cases, the rank is equal to the strength of the design.

Definition 3.2.3. (Ucycle of rank two) Let $(V, \mathcal{B})$ be $a(v, K, \lambda)-P B D$. Let $b=|\mathcal{B}|$ and let each $B \in \mathcal{B}$, be represented by a sequence $\left\langle x_{0}, x_{1}\right\rangle$, where, for $i=1,2, x_{i} \in B$. 


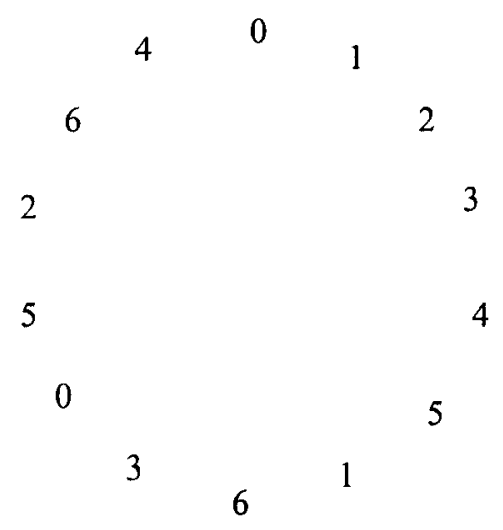

Figure 13: A Ucycle of rank three for the blocks of a TTS(7)

The sequence $\mathrm{U}=a_{0}, a_{1}, \ldots, a_{b-1}$ is a Ucycle for the $(v, K, \lambda)-P B D$ if $\left\langle a_{i}, a_{i+1}\right\rangle$, $0 \leq i \leq b-1$, runs through the representation of each element of $\mathcal{B}$ exactly once, where index addition is performed modulo $b$.

Notice that in this definition the representation of blocks is not unique. That is, there are $\left(\begin{array}{l}k \\ 2\end{array}\right)$ ways to represent a block of size $k$. In fact, taking order into account means that there are $2\left(\begin{array}{l}k \\ 2\end{array}\right)$ ways to represent a block of size $k$. Furthermore, when $\lambda>1,\left\langle x_{0}, x_{1}\right\rangle$ will appear in $\lambda$ different blocks, therefore, each pair of consecutive points in the Ucycle does not represent a unique block. We will address these issues in Section 4.2.

\subsection{Configuration Ordering}

While the definitions of Gray codes and Ucycles can be naturally extended to blocks of designs, ordering criteria based on the structures formed by consecutive blocks 
are also natural. Configurations allow us to discuss the structures formed by sets of blocks. The majority of research regarding configurations has focused on the presence or absence and the enumeration of given configurations in certain families of designs. For example, anti-Pasch triple system existence has been settled. The other most commonly studied aspect of configurations is the question of whether or not a given design can be decomposed into copies of a given configuration. In 2003, another area of investigation regarding configurations was identified [15]. Cohen and Colbourn's configuration ordering is a listing of the blocks of a design such that every set of consecutive blocks of the necessary number is isomorphic to a given configuration. The requirements for existence of a configuration ordering are stronger than for existence of a decomposition of a design into copies of the same configuration. That is, any configuration ordering for a design gives a decomposition of that design into copies of the configuration in question. The main question asked is, given a configuration, does a design admit a configuration ordering of its blocks? In this section, we introduce the necessary background on configuration ordering. We define a generalized configuration ordering involving sets of configurations and we translate some known results into this language. 


\subsubsection{Ordering the Blocks of Steiner Triple Systems for Ap- plication to Erasure-Correcting Codes}

Configuration orderings were initially introduced by Cohen and Colbourn in relation to erasure codes [15]. Parallel disk architectures are a common feature in today's computing environment. These systems, known as Redundant Arrays of Independent Disks (RAID), connect many disks together in an attempt to improve I/O performance. While connecting several disks increases $\mathrm{I} / \mathrm{O}$ performance, it has a detrimental effect on the reliability of the system - more disks in the system increases the likelihood that some part of the system (a disk) will fail. When a disk suffers a catastrophic failure its data is deemed unreadable, therefore, such a disk failure is called an erasure. Erasure-correcting codes are designed to recover the erased data when the location of the erasure is known.

The two key factors considered in the evaluation of RAID systems are performance and reliability. The performance of a system is usually judged by examining system response time. The reliability of a system refers to the ability of the system to restore the contents of erased disks. The reliability of a parallel system of disks can be improved by introducing redundancy. As this redundancy often takes the form of parity calculations, the performance of the system is adversely affected, thus, when designing these redundant operations it is important to reduce the negative impact 
on performance. An $[\boldsymbol{n}, \boldsymbol{c}, \boldsymbol{k}]$-erasure-correcting code consists of an encoding algorithm $\varepsilon$ and a decoding algorithm $\delta$ with the following properties. Given an $n$-tuple of data $S$ (for $n$ disks), $\varepsilon$ produces an $(n+c)$-tuple $\varepsilon(S)=\left(\varepsilon_{1}(S), \varepsilon_{2}(S), \ldots, \varepsilon_{n+c}(S)\right)$ called a codeword, such that for any $U \subseteq\{1,2, \ldots, n\}$, where $|U|=n+c-k$, the decoding algorithm $\delta$ is able to recover $S$ from $\left(U,\left\{\varepsilon_{i}(S): i \in U\right\}\right)$ [14]. We will refer to $\boldsymbol{k}$-erasure-correcting codes when we do not wish to specify $n$ and $c$. An $[n, c, k]$-erasure-correcting code that produces codewords in which the first $n$ bits are simply $S$ (the unmodified original data) is called systematic. The remaining bits are called the checks. We consider only systematic codes in this section. To apply an $[n, c, k]$-erasure-correcting code to an array of disks, the array of disks is broken into two groups by designating the first $n$ disks to be information disks and others to be check disks. Information disks contain the original, unmodified data. Check disks contain an encoding of the original data determined by parity operations. The above definition of an $[n, c, k]$-erasure-correcting code indicates that the system can tolerate $k$ erasures. To determine an upper bound on $k$, suppose that $c+1$ disks fail. If these disks are the $c$ check disks and one of the $n$ information disks it is impossible to recover the erased information. Therefore, we must have $k \leq c$.

In order to discuss the performance of a disk array we introduce the following terms. The check disk overhead is the ratio of the number of check disks to the number of information disks $(c / m)$. The update penalty is the number of check 
disks whose contents must be changed when a minimal change is made to the contents of a given information disk. For an erasure-correcting code to tolerate $k$ erasures, every update must affect the contents of at least $k+1$ disks (one information disk and $k$ check disks), therefore, the update penalty of a $k$-erasure-correcting code is at least $k$. Finally, the group size is the number of disks that must be accessed during reconstruction of the contents of a single failed disk. We wish each of these values to be as small as possible. Because update penalties are most important to the performance of a code, we will assume from now on that we are looking at $k$-erasure codes with update penalty $k$.

An $[n, c, k]$-erasure-correcting code can be described in terms of a $c \times(n+c)$ matrix over $\mathbb{F}_{2}$. Suppose $H=[P \mid I]$ is the matrix of an $[n, c, k]$-erasure-correcting code. $P$ is the $c \times n$ matrix that determines the relationship between the check and information disks and $I$ is the $c \times c$ identity matrix. We identify the columns of $P$ with the $n$ information disks and the columns of $I$ with the $c$ check disks. This matrix is called the parity check matrix of the code.

Let us examine a particular erasure-correcting code in order to solidify the ideas introduced thus far. The full-2 code, represented by the $c \times\left(\left(\begin{array}{l}c \\ 2\end{array}\right)+c\right)$ matrix $H_{\text {full } 2}=$ $\left[P_{\text {full } 2} \mid I\right]$, is a 2-erasure-correcting code. The columns of $P_{f u l l 2}$ are all possible distinct $c$-tuples of weight two. For any positive integer $k$, we can define a full- $k$ code in a similar fashion. $H_{f u l l 2}$ for $c=5$ is given below 


$$
\boldsymbol{H}_{\text {full } 2}=\left[\begin{array}{ccccccccccccccc}
1 & 1 & 1 & 1 & 0 & 0 & 0 & 0 & 0 & 0 & 1 & 0 & 0 & 0 & 0 \\
1 & 0 & 0 & 0 & 1 & 1 & 1 & 0 & 0 & 0 & 0 & 1 & 0 & 0 & 0 \\
0 & 1 & 0 & 0 & 1 & 0 & 0 & 1 & 1 & 0 & 0 & 0 & 1 & 0 & 0 \\
0 & 0 & 1 & 0 & 0 & 1 & 0 & 1 & 0 & 1 & 0 & 0 & 0 & 1 & 0 \\
0 & 0 & 0 & 1 & 0 & 0 & 1 & 0 & 1 & 1 & 0 & 0 & 0 & 0 & 1
\end{array}\right]
$$

The first ten columns are associated with the ten information disks, while the last five columns are associated with the five check disks. The contents of check disk $i$ is an encoding of the contents of the information disks whose corresponding columns in $P_{\text {full } 2}$ have a one in row $i$. For example, the 1 in the $11^{\text {th }}$ column of $H_{\text {full } 2}$ means that the first check disk stores an encoding of the data in the $1^{\text {st }}, 2^{\text {nd }}, 3^{\text {rd }}$ and $4^{\text {th }}$ information disks. Note that each column has weight two meaning that each information disk has its data encoded on two check disks. The full-2 code is 2-erasurecorrecting because if any two disks fail the data stored may be restored. However, if three disks fail (for example, the first information disk and the first two check disks) the data cannot be recovered. In general, the full-2 code has a check disk overhead of $c /\left(\begin{array}{l}c \\ 2\end{array}\right)=2 /(c-1)$, a group size of $c-1$, and an update penalty of 2 . As noted earlier, update penalties are the primary performance cost associated to an erasure-correcting code. When the update penalty is assumed to be minimized, the next greatest factor in diminishing performance is due to writing data to consecutive information disks. This involves updating some number of check disks. Let $u_{s}$ denote the average number 
of check disks updated when $s$ consecutive information disks are written to. Cohen and Colbourn have focused on minimizing $u_{s}$ through ordering of information disks within an established system. The remainder of this section presents some of their results.

The full-2 code with $c$ check disks can be represented by the complete graph $K_{c}$. Each vertex of $K_{c}$ represents a check disk and the edges represent information disks (you can read these edges off the columns of $H_{f u l l 2}$ ). As mentioned above, Hellerstein et al. have shown that a code with minimum update penalty two is obtained by having each information disk "checked" by two check disks, with no two information disks sharing the same pair of check disks [31]. Therefore, any 2-erasure-correcting code can be represented by a graph with vertices representing check disks and an edge connecting two check disks representing the information disk they "check". Let $G=(V, E)$ be a graph with $m$ edges. Let $d$ be a fixed positive integer less than $m$ which represents the number of disks involved in a typical write. Call $d$ the window. An edge-ordering of $G$ is a permutation of the edge indices $\{0,1, \ldots, m-1\}$. Suppose $G$ is a graph with edge-ordering $\pi$ and window d. For $0 \leq i \leq m-d$, define graphs $G_{i}^{\pi, d}$ to be the subgraphs of $G$ induced by the edges $\left\{e_{\pi(i)}, e_{\pi(i+1)}, \ldots, e_{\pi(i+d-1)}\right\}$. The cost of updating the disks represented by the subgraph induced by $d$ consecutive edges is equal to the sum of the number of edges and the number of vertices of non-zero degree in the subgraph. As $d$ (the number of 
edges) is fixed, it is clear that the only way to reduce this access cost is to reduce the number of vertices induced. Let $n_{i}^{\pi, d}$ represent the number of vertices of non-zero degree in $G_{i}^{\pi, d}$ and define the $\boldsymbol{d}$-access cost of graph $G$ under the ordering $\pi$ to be

$$
\frac{\sum_{i=0}^{m-d} n_{i}^{\pi, d}}{(m+1-d)} .
$$

When an ordering $\pi$ minimizes the d-access cost over all edge orderings of $G$ it is called a $\boldsymbol{d}$-optimal ordering of $G$. When an ordering $\pi$ maximizes the d-access cost over all edge orderings of $G$ it is called a $d$-pessimal ordering of $G$.

The task of reducing the number of check disks accessed in a typical write becomes a problem of reducing the number of vertices induced by any $d$ consecutive edges of an edge-ordering. For example, if $d=3$ the minimum number of vertices induced by three edges is three, which occurs when the edges form a triangle. The maximum number of vertices induced by three edges is six, which occurs when all edges are disjoint. Therefore, to obtain a 3-optimal ordering, we would like to find an edgeordering where every consecutive set of three edges induces a triangle. This is not possible, unless there are only three vertices in the graph, because the graph does not have double edges. As triangles cannot be consecutively induced, the best we can hope for is to alternate between inducing three vertices and inducing four vertices. Whether or not this is possible is determined by the answer to the following question. When can the edges of the complete graph $K_{c}$ be ordered by a permutation $\pi$ so that 
among the $\left(\begin{array}{l}c \\ 2\end{array}\right)-2$ subgraphs $G_{i}^{\pi, 3}$, at least $\frac{1}{4}\left(c^{2}-c-6\right)=\frac{1}{2}\left[\left(\left(\begin{array}{l}c \\ 2\end{array}\right)-2\right)-1\right]$ subgraphs form triangles? Such an ordering of edges is called a ladder ordering of pairs. A ladder ordering is called circular if for any three consecutive edges, $e_{i}, e_{i+1}, e_{i+2}$, the subgraph induced by these edges alternates between having three vertices and having four vertices, with addition of subscripts performed modulo $\left(\begin{array}{l}c \\ 2\end{array}\right)$. A circular ordering can only exist when $c \equiv 0,1(\bmod 4)$, which ensures that two consecutive triangles are not induced.

Theorem 3.3.1. ([16]) A ladder ordering of pairs for $K_{c}$ exists for all admissible $c$, except possibly when $c \in\{15,18,22\}$.

Recall that a $(p, \ell)$-configuration is a set system with $p$ elements and $\ell$ blocks in which every element is contained in at least one block. Let $C$ be a configuration. In [15], Cohen and Colbourn ask: "When does there exist a Steiner triple system of order $v$ in which the triples can be ordered such that every $\ell$ consecutive triples form a configuration isomorphic to $C$ ?" Such an ordering is called a $C$-ordering for the STS.

Definition 3.3.1. (Configuration Ordering) Let $S=(V, \mathcal{B})$ be a $B I B D$ with $|\mathcal{B}|=b$. Let $C$ be a configuration on $\ell$ blocks. A C-ordering for $S$ is a list of the blocks of $S,\left[B_{0}, B_{1}, \ldots, B_{b-1}\right]$, with the property that $B_{i}, B_{i+1}, \ldots, B_{i+\ell-1} \cong C$, for all $0 \leq i \leq b-\ell$. If $B_{i}, B_{i+1}, \ldots, B_{i+\ell-1} \cong C$ holds for all $0 \leq i \leq b-1$, with subscript addition performed modulo $b$, then the ordering is called $\boldsymbol{C}$-cyclic. 
Cohen and Colbourn have investigated the effect of different orderings of the columns of the parity check matrix induced by configurations on the performance of RAID systems. Let $D_{\ell}$ be the $(3 \ell, \ell)$-configuration consisting of $\ell$ disjoint triples. Cohen and Colbourn call a $D_{\ell^{-}}$-ordering for an STS a pessimal ordering. Note that a cyclic $D_{2}$-ordering of an STS is equivalent to a Hamilton cycle in the complement of the block intersection graph of the STS.

Theorem 3.3.2. ([15]) For all admissible $v \geq 9 \ell-6$, there exists an $S T S(v)$ with a $D_{\ell}$-ordering.

Theorem 3.3.3. ([15]) Let $\ell$ be a positive integer. For all admissible $v \geq 81(\ell-1)+1$, every $S T S(v)$ admits a $D_{\ell}$-ordering.

Proof. Suppose $S=(V, \mathcal{B})$ is an $\operatorname{STS}(v)$. Let $G$ be the 1-block intersection graph of $S$. $G$ is regular of degree $3(r-1)=3(v-3) / 2$. By Brookes' Theorem, $G$ has a proper vertex colouring in $s \leq 3(v-3) / 2$ colours. Partition the vertices of $G$ by colour class, letting $R_{i}$ represent the set of vertices having colour $i$. Assume $\left|R_{i}\right| \leq\left|R_{i+1}\right|$ for all $1 \leq i<s$. If $3\left|R_{1}\right|<\left|R_{s}\right|$, there is a triple in $R_{s}$ that intersects no triple of $R_{1}$. Move that triple from $R_{s}$ to $R_{1}$, re-labeling the $R_{i}$ if necessary to maintain $\left|R_{i}\right| \leq\left|R_{i+1}\right|$. Repeat this process until $3\left|R_{1}\right| \geq\left|R_{s}\right|$.

The total number of triples in $S$ is $v(v-1) / 6$, therefore

$$
\sum_{i=1}^{s}\left|R_{i}\right|=\frac{v(v-1)}{6}
$$


Since the partitions are ordered by increasing size and $R_{s}$ is the last partition in the order, we have $\left|R_{s}\right| \geq\lceil v / 9\rceil$. That is, the size of $R_{s}$ is greater than or equal to the average size of a partition, which is $v(v-1) /(6 s)$. Using the relation established between $R_{1}$ and $R_{s}$, we obtain $\left|R_{1}\right| \geq\lceil v / 27\rceil$. Since $v \geq 81(\ell-1)+1$ we have

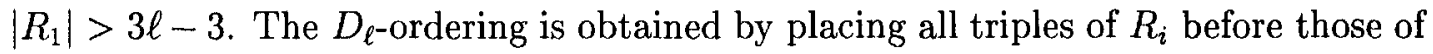
$R_{i+1}$ for all $1 \leq i<s$. Within classes, order the triples as follows. Order the triples of $R_{1}$ arbitrarily and suppose that $R_{1}, \ldots, R_{i-1}$ have been ordered. When selecting the $j^{\text {th }}$ triple of $R_{i}$ it is necessary to ensure that it does not intersect any of the last $\ell-j$ triples of $R_{i-1}$. Since $j-1$ triples have already been chosen from $R_{i}$, and the total number of triples in $R_{i}$ that may intersect the last $\ell-j$ triples of $R_{i-1}$ is at most $3(\ell-j)$, there are at most $(j-1)+3(\ell-j)=3 \ell-2 j-1$ triples of $R_{i}$ that we cannot use. However, there are at least $3 \ell-2$ triples in $R_{i}$, so there exists a triple that can be placed in the $j^{\text {th }}$ position in the ordering of $R_{i}$.

Theorems 3.3.2 and 3.3.3 provide some insight into bad orderings for the columns of the parity check matrix, however, Cohen and Colbourn were most interested in improving the performance of disk arrays and therefore, we now focus our attention on good orderings. A good ordering minimizes the number of check disks associated with $\ell$ consecutive triples. Suppose $\ell=2$. The $(5,2)$-configuration consisting of two intersecting triples is denoted by $A_{2}$. An $A_{2}$-ordering is called an optimal ordering. Theorem 3.3.4 follows from the fact that the 1-block intersection graph of an $\operatorname{STS}(v)$ 
is Hamiltonian.

Theorem 3.3.4. ([33]) Every STS(v) admits a cyclic $A_{2}$-cyclic ordering.

$B_{5}$ is the $(6,3)$-configuration shown in Figure 14 .

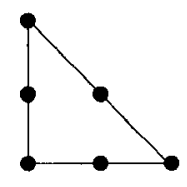

Figure 14: $B_{5}$

When $\ell=3$, a $B_{5}$-ordering is an optimal ordering. The Fano plane (shown in Figure 3 on page 18) admits a $B_{5}$-cyclic ordering: $\{0,1,3\},\{1,2,4\},\{0,2,6\},\{1,5,6\}$, $\{2,3,5\},\{3,4,6\},\{0,4,5\}$.

Theorem 3.3.5. ([15]) For each admissible $v \geq 15$, there exists an $S T S(v)$ which is not $B_{5}$-orderable.

Despite the statement of Theorem 3.3.5, Cohen and Colbourn expect that for every admissible $v \geq 15$, there is a $B_{5}$-orderable $\operatorname{STS}(v)$. In [15] they establish the existence of some small $B_{5}$-orderable STSs and hope that these orderings can be used in creating $B_{5}$-orderings for larger systems.

\subsubsection{Translated Results in Configuration Ordering}

In this section, we translate results from other areas of combinatorics into the language of configuration ordering. We will see that there are a significant number of results 
in this area; however, there remain gaps to be filled. The number of results that have natural expressions as configuration orderings demonstrates how intuitive this type of block ordering is.

The following result, translated from minimal change combinatorial Gray codes, applies to the family of designs consisting of all $k$-subsets of an $n$-set. These are simple designs with $|\mathcal{B}|=\left(\begin{array}{l}v \\ k\end{array}\right)$.

Theorem 3.3.6. $([5,7,11,23,45,59])$ For $k \geq 2$, every $\left(v, k,\left(\begin{array}{c}v-2 \\ k-2\end{array}\right)\right)$-BIBD admits $a(k+2,2)$-cyclic ordering.

The following result comes from pair designs. A pair design is an ordering of the $\left(\begin{array}{l}n \\ 2\end{array}\right)$ pairs of $n$ elements such that no two consecutive occurrences of an element have fewer than $\lfloor(n-3) / 2\rfloor$ pairs separating them.

Theorem 3.3.7. $[29,49] A(v, 2,1)$-BIBD, with $v>4$, admits a $(2 d, d)$-ordering if and only if $d \leq\lfloor(v-3) / 2\rfloor$.

Staying with $(v, 2,1)$-BIBDs, the next two results follow from basic graph theory.

Theorem 3.3.8. Every $(v, 2,1)$-BIBD admits a $(3,2)$-cyclic ordering.

The pair-adjacency graph for a $(v, 2,1)$-BIBD is $K_{v}$. In fact, since the block size is two, each edge of $K_{v}$ represents a block. The line graph of a graph $G=(V, E)$ is the graph with vertex set $E$ and an edge between vertices $e_{1}$ and $e_{2}$ if the edges in $G$ represented by these vertices share a common endpoint. The line graph of $K_{v}$ is 
Hamiltonian. Theorem 3.3.8 follows from the fact that a Hamilton cycle in the line graph is a listing of all edges of $K_{v}$ (i.e. all blocks of the $(v, 2,1)$-BIBD) such that consecutive edges share a common vertex.

The next theorem follows from the fact that $K_{v}$ is Eulerian if and only if $v$ is odd.

Theorem 3.3.9. Every $(v, 2,1)-B I B D$, with $v$ odd, admits $a\left\{(3,3),(4,3)_{1}\right\}$-cyclic ordering, where the $(4,3)_{1}$-configuration is the 4-path.

In 1989, Graham asked: is the block intersection graph of an STS $(v)$ Hamiltonian? Several authors have addressed the generalization of this question: is the 1-block intersection graph of a strength two design Hamiltonian? The answers to these questions lead to several results in configuration ordering because the 1-block intersection graph of a design having a Hamilton cycle is equivalent to the design admitting a configuration ordering in which every pair of consecutive blocks intersects in exactly one point.

Theorem 3.3.10. ([33]) Every $(v, k, 1)$-BIBD admits a $(2 k-1,2)$-cyclic ordering.

Theorem 3.3.11. ([32]) Every $(v, 3, \lambda)$-BIBD, with $v \geq 12$, admits a $(5,2)$-cyclic ordering.

Theorem 3.3.12. ([2]) Every $(v, K, 1)-P B D$, with $\max (K) \leq 2 \cdot \min (K)$, admits a cyclic ordering in which every pair of consecutive blocks intersects in exactly one point. 
Hare improved Theorem 3.3.12 by proving that the 1-block intersection graph of every $(v, K, 1)$-PBD, with $\min (K) \geq 3$, is edge-pancyclic [30]. A graph $G$ is edgepancyclic if for every edge $e \in E(G)$ and every integer $n, 3 \leq n \leq|V(G)|$, there is a cycle of length $n$ in $G$ containing $e$.

Theorem 3.3.13. ([30]) Every $(v, K, 1)-P B D$, with $\min (K) \geq 3$, admits a cyclic ordering in which every pair of consecutive blocks intersects in exactly one point.

M. Colbourn and Johnstone have looked at the existence of minimal change orderings for certain twofold triple systems. A minimal change ordering of triples where consecutive triples differ in a single element is equivalent to the existence of an $A_{3}$ ordering for the triples.

Theorem 3.3.14. ([20]) Every simple TTS(v), with $v \leq 10$ admits an $A_{3}$-ordering.

The main discussion in [20] is focused on the non-existence of minimal change orderings. The authors give a construction for TTS $(v)$ s that admit no minimal change ordering for their blocks. This construction is used to create a TTS(19) with no minimal change ordering; however, the correctness of the construction is not proved for general $v$. The following conjecture is implied by the construction in [20] but not explicitly stated.

Conjecture 3.3.15. There exists $v_{0} \in \mathbb{N}$ such that for all admissible $v \geq v_{0}$, at least one $T T S(v)$ does not admit an $A_{3}$-ordering. 


\subsubsection{Generalized Configuration Ordering}

We define a natural generalization of configuration ordering.

Definition 3.3.2. (C-ordering) Let $\mathcal{C}$ be a set of configurations, each having $\ell$ blocks. $A \mathcal{C}$-ordering for a design is a listing of the blocks of the design such that every $\ell$ consecutive blocks form a configuration isomorphic to a configuration in $\mathcal{C}$.

Let $S=(V, \mathcal{B})$ be a triple system with $|\mathcal{B}|=b$. Let $\mathcal{C}$ be a set of configurations, each having $\ell$ triples. A $\mathcal{C}$-ordering for $S$ is a list $\left[B_{0}, B_{1}, \ldots, B_{b-1}\right]$ with the property that for all $0 \leq i \leq b-\ell$ there exists $C_{j} \in \mathcal{C}$ such that $B_{i}, B_{i+1}, \ldots, B_{i+\ell-1} \cong C_{j}$. If $B_{i}, B_{i+1}, \ldots, B_{i+\ell-1} \cong C_{j}$ holds for all $0 \leq i \leq b-1$, with subscript addition performed modulo $b$, then the ordering is called $\mathcal{C}$-cyclic. We will say that a design admitting at least one such ordering is $\mathcal{C}$-cyclic orderable.

There exist several results which can be translated into this language. The ladder ordering of pairs, due to Cohen and Colbourn, discussed in Section 3.3.1, is equivalent to the following statement with the set of $\{(3,3),(4,3)\}$-configurations illustrated in Figure 15.

Theorem 3.3.16. ([16]) Every $(v, 2,1)$-BIBD admits a $\{(3,3),(4,3)\}$-ordering.

Recall Hare's result that says the 1-block intersection graph of every $(v, K, 1)$ $\mathrm{PBD}$, with $\min (K) \geq 3$, is edge-pancyclic. Instead of considering consecutive pairs of blocks in a Hamilton cycle of the 1-block intersection graph, consider consecutive 


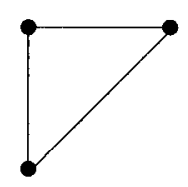

$(3,3)$

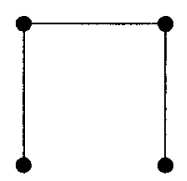

$(4,3)$

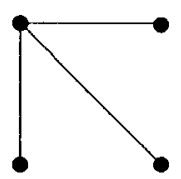

$(4,3)$

Figure 15: The $(3,3)$ - and $(4,3)$-configurations

triples. Let $\mathcal{C}_{h}$ denote the set of configurations shown in Figure 16. Hare's result is equivalent to the following theorem.

Theorem 3.3.17. ([30]) Every $(v, K, 1)-P B D$, with $\min (K) \geq 3$, admits a $\mathcal{C}_{h}$-cyclic ordering.
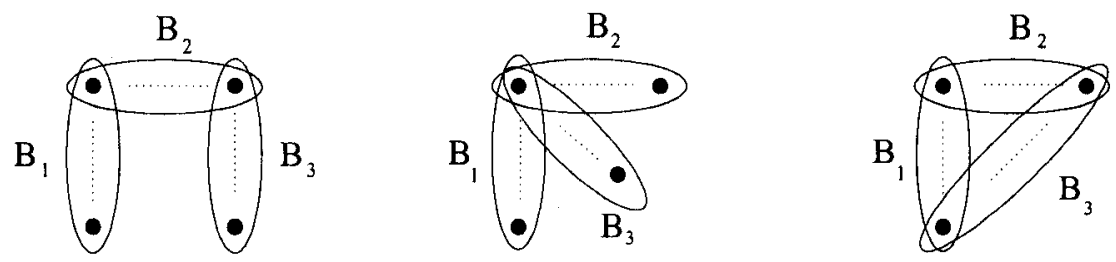

Figure 16: The set of configurations in the translation of Hare's edge-pancyclicity result to configurations of three blocks

In this chapter, we have established several rubrics under which to formulate questions regarding ordering the blocks of designs. In the next two chapters, we present results for each and discuss relationships between these definitions of ordering. 


\section{Chapter 4}

\section{Gray Codes and Universal Cycles}

\section{for Designs}

This chapter and the next present new results on ordering the blocks of designs. In this chapter we consider Gray codes and Ucycles for block designs. The analysis will be broken into two sections: twofold triple systems (TTSs) and balanced incomplete block designs (BIBDs). In addition to proving the existence of rank two and rank three Ucycles for certain designs, we discuss how these results relate to configuration orderings. In Chapter 5, we will prove some configuration ordering results directly. 


\subsection{Gray Codes and Universal Cycles for TTSs}

Twofold triple systems are a natural family with which to begin our investigation because the index and the strength of the design are both one less than the block size. Furthermore, the small size of the blocks makes it easy to look directly at the points in each block of the design. A minimal change ordering for a TTS is a listing of the blocks of the design such that consecutive blocks differ in one entry. Such a minimal change ordering can be realized in two ways. As a Gray code this is directly analogous to minimal change combinatorial Gray codes for $k$-subsets of $[n]$. As a rank three Ucycle this is similar in appearance to a Ucycle for $k$-subsets of $[n]$, although the construction methods are very different.

As noted in Chapter 2, a $\operatorname{TTS}(v)$ exists if and only if $v \equiv 0,1(\bmod 3)$. Lemma 3.2.3 (page 82) implies that if there exists a rank three Ucycle for a TTS $(v)$, then three must divide $r$, where $r=v-1$ in this case. This implies that $v$ must be equivalent to one modulo three, therefore, $v \equiv 1,4,7,10(\bmod 12)$ are the only values of $v$ for which a $\operatorname{TTS}(v)$ may admit a Ucycle of rank three. For all $v \equiv 0,1(\bmod 3)$ a 2 intersecting Gray code for a $\operatorname{TTS}(v)$ may exist. In this section, we prove that for $v \equiv 1,3,4 ; 7(\bmod 12)$, with sporadic exceptions, there exists a TTS $(v)$ that admits a 2 -intersecting Gray cycle for its blocks. When $v \equiv 1,4,7(\bmod 12)$, this ordering can be written as a Ucycle of rank three. Determining the existence of rank three Ucycles for $\operatorname{TTS}(v) \mathrm{s}$ with $v \equiv 10(\bmod 12)$, and the existence of 2-intersecting Gray 
cycles for $\operatorname{TTS}(v)$ s with $v \equiv 0,6,9(\bmod 12)$, remains an open problem.

The existence of a 2-intersecting Gray cycle for the blocks of a TTS is equivalent to the existence of a Hamilton cycle in the 2-block intersection graph of the design. It is also equivalent to saying that the blocks admit an $A_{3}$-ordering (Figure 1 on page 7). In [20], M. Colbourn and Johnstone construct a TTS having a 2-block intersection graph that does not admit a Hamilton cycle. In proving this result, M. Colbourn and Johnstone first establish the following lemma.

Lemma 4.1.1. ([20]) Given a TTS(v), each component of the corresponding 2-block intersection graph is 3-connected.

The main result of [20] is the determination of a TTS which has no minimal change presentation, that is, has no Hamilton cycle in the 2-block intersection graph of the design. An obvious condition for existence of a Hamilton cycle in a graph is that the graph must be connected, therefore, M. Colbourn and Johnstone deal only with TTSs that are simple. By this they mean that the TTS has no repeated blocks and the TTS has no non-trivial subdesign. Using an approach due to McCuaig, M. Colbourn and Johnstone present a construction for an infinite family of graphs which can be viewed as 2-block intersection graphs of TTSs. Let $G=(V, E)$ be a cubic, 3-connected graph that does not contain a Hamilton cycle. A good example of such a graph is the Petersen graph. Let $v \in V$ and let the three neighbours of $v$ be $x, y, z \in V$. Create three copies of $G \backslash\{v\}$ and connect them as shown in Figure 17, then add any graph 
$H$ such that the new graph created can be labeled as the 2-block intersection graph of some TTS. This new graph does not contain a Hamilton cycle. The authors claim that such an $H$ exists, however they give only one example, choosing the Petersen graph for $G$ and choosing $H$ such that the resulting graph can be labeled as the 2block intersection graph of a TTS(19). The authors do not prove or discuss whether it is possible to find other graphs $H$ which complete the graph in Figure 17 (with $G$ the Petersen graph) to the 2-block intersection graph of a TTS, nor do they indicate whether, given an arbitrary $G$ with the required properties, it is always possible to find an $H$ which completes the copies of $G$ to the 2-block intersection graph of some TTS. The existence of $H$ is worth investigating as M. Colbourn and Johnstone note that there exist many cubic 3-connected graphs which have no corresponding TTS or partial TTS labeling.

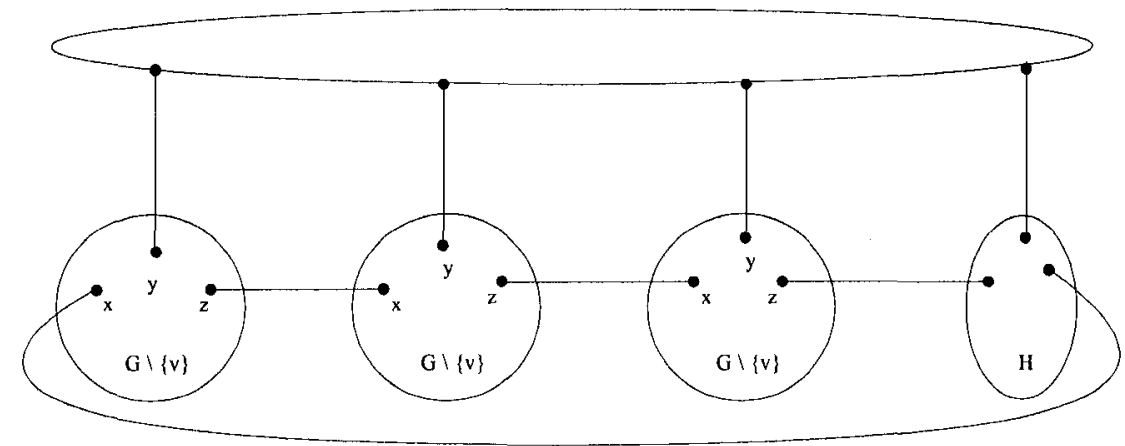

Figure 17: The 2-block intersection graph of a TTS having no Hamilton cycle

While M. Colbourn and Johnstone prove the existence of a TTS having no minimal change representation, they predict that for all admissible $v$ there do exist 
minimal change $\operatorname{TTS}(v) \mathrm{s}$. We prove there exists a $\operatorname{TTS}(v)$, for each $v \equiv 1,3,4,7$ (mod 12), with sporadic exceptions, that admits a minimal change representation and, for $v \equiv 1,4,7(\bmod 12)$, we are able to prove that the stronger Ucycle representation exists. The existence of a minimal change ordering for at least one TTS of each admissible order is perhaps the best general result we can hope for, as $M$. Colbourn and Johnstone's work implies that we will not be able to prove that all $\operatorname{TTS}(v) \mathrm{s}$ admit minimal change orderings.

We prove the existence of Gray cycles and Ucycles for the blocks of twofold triple systems in very similar ways. In fact, we think of Gray cycles as Ucycles that cannot be completed unless the change rule is relaxed in some places. Define a partial Ustring to be a sequence having Ucycle properties (although possibly not cyclic) that represents some, but not all, blocks of a design. To create a Ucycle for a design, we join partial Ustrings, which together represent all blocks of the design, so that the Ucycle property holds across joins. To create a Gray cycle for a design we take collections of partial Ustrings, which together contain all blocks of the design, and join them so that the minimal change property holds across joins.

Suppose $U$ is a partial Ustring containing some blocks of a $\operatorname{TTS}(v), S=(V, \mathcal{B})$. What properties must $U$ have? First, the list of blocks induced by $U$ will have the 
property that the intersection of four consecutive blocks is empty and the intersection of three consecutive blocks is exactly one. This is due to the fact that consecutive blocks are "picked off" $U$ by shifting right by one entry. For example, suppose $v, w, x, y, z$ is a partial Ustring. The blocks represented by this partial Ustring are $\{v, w, x\},\{w, x, y\}$ and $\{x, y, z\}$. We cannot have three consecutive blocks intersecting in more than one point because every pair of points appears in exactly two blocks of a TTS. On the other hand, every point in $U$ appears in exactly three blocks unless $U$ is not cyclic (in which case the endpoints of $U$ appear in fewer blocks). Second, a complete Ucycle must have the property that all pairs $\{x, y\} \in V \times V$ either appear adjacent in the Ucycle exactly once (either as $x, y$ or $y, x$ ) or appear exactly twice at distance two (either as $x,-, y$ or $y,-, x$ ). Consider the partial Ustring in the example above. In addition to the block $\{w, x, y\}$ there is exactly one other block in $\mathcal{B}$ containing the pair $\{w, y\}$. The other block must appear in the Ucycle as $w,-, y$ or $y,-, w$, because a consecutive occurrence of $w$ and $y$ would result in two additional blocks containing this pair. These two properties govern the creation of partial Ustrings for TTSs.

For each admissible order $v$, there exists a $\operatorname{TTS}(v)$ that is either cyclic or 1rotational. Recall that a cyclic design of order $v$ has automorphism $\pi: i \longmapsto i+1$ $(\bmod v)$, while a 1-rotational design of order $v$ has an automorphism with one fixed point and a cycle of length $v-1$. The fixed point is denoted $\infty$ and the automorphism 
is $\pi: i \longmapsto i+1(\bmod v-1)$. Cyclic $\operatorname{TTS}(v) \mathrm{s}$ exist for all $v \equiv 0,1,3,4,7,9(\bmod 12)$, except $v=9$, while 1 -rotational $\operatorname{TTS}(v) \mathrm{s}$ exist for all $v \equiv 0,1(\bmod 3)$. We use the structural properties of such designs to construct partial Ustrings. Recall that a supplementary difference set, $\mathcal{S}$, for a cyclic $(v, k, \lambda)$-BIBD must have the following properties: (1) $|S|=k$, for all $S \in \mathcal{S}$, and (2) the collection $\{x-y: x, y \in S, S \in$ $\mathcal{S}, x \neq y\}$ must contain every element of $\{1,2, \ldots, v\}$ exactly $\lambda$ times. Each set $S \in \mathcal{S}$ yields a base block, and when all base blocks are developed through $\mathbb{Z}_{v}$ they generate the design. The collection of base blocks for a 1-rotational $(v, k, \lambda)$-BIBD must each be of size $k$ and together must contain the differences $\{1,2, \ldots, v-2\}$ exactly $\lambda$ times. Note that while the point $\infty$ will be in some base blocks, the differences involving this fixed point are ignored. Given a collection of blocks, these two difference properties can be used to determine if the blocks form a set of base blocks for a cyclic or 1rotational design.

The differences associated to the base blocks of a cyclic $(v, k, \lambda)$-BIBD reside in $\mathbb{Z}_{v} \backslash\{0\}$. When determining the appearance of each of these differences in the base blocks of a design, we will work with the set $\{-(\lceil v / 2\rceil-1),-(\lceil v / 2\rceil-2), \ldots,\lfloor v / 2\rfloor\}$ which is equivalent to $\mathbb{Z}_{v}$. Notice that if the difference $x-y$ appears in a base block then so does the difference $v-(x-y) \equiv y-x(\bmod v)$, therefore, we need only ensure that each difference $d \in\{1,2, \ldots,\lfloor v / 2\rfloor\}$ appears $\lambda$ times in the base blocks of a cyclic $(v, k, \lambda)$-BIBD. In fact, the appearance of $d$ or $-d$, for $d \in\{1,2, \ldots,\lfloor v / 2\rfloor\}$, 
in a base block contributes to the count of the total number of times the difference $d$ appears in the base blocks in question. Similarly, the differences associated to the base blocks of a 1 -rotational $(v, k, \lambda)$-BIBD reside in $\mathbb{Z}_{v-1} \backslash\{0\}$. In this case we need only ensure that each difference $d \in\{1,2, \ldots,\lfloor(v-1) / 2\rfloor\}$ appears $\lambda$ times in the base blocks of a 1-rotational $(v, k, \lambda)$-BIBD.

To prove the existence of rank three Ucycles and 2-intersecting Gray cycles for certain TTS $(v)$ s, we will first construct partial Ustrings that contain all base blocks of the TTS in question. To construct partial Ustrings we must define a new structure and introduce some new notation. Let $D_{v}=d_{0}, d_{1}, \ldots, d_{\ell-1}$ be a sequence of elements from $\mathbb{Z}_{v} \backslash\{0\}$ such that $d_{i} \neq d_{j}(\bmod v)$ when $i \neq j$. Let $\sigma_{D_{v}}$ be the sequence of sums of pairs of consecutive elements in $D_{v}$, that is, $\sigma_{D_{v}}=d_{0}+d_{1}(\bmod v), d_{1}+d_{2}$ $(\bmod v), \ldots, d_{\ell-2}+d_{\ell-1}(\bmod v)$. When this sequence of sums includes the sum of the last and first elements in $D_{v}$ denote this sequence $\sigma_{D_{v}}^{\prime}=\sigma_{D_{v}}, d_{\ell-1}+d_{0}(\bmod v)$. For the remainder of this section, all operations involving elements of $D_{v}$ will be assumed to be done modulo $v$. Given a sequence $S$, let $\{S\}$ denote the collection of elements appearing in $S$ (including repeated elements) and let $\pm\{S\}$ denote the collection of elements in $S$ and the negative of these elements. Let $x\{S\}$, for $x \in \mathbb{Z}^{+}$, denote the collection of elements appearing in $S$, with each element repeated $x$ times.

Definition 4.1.1. (Cyclic Difference Sequence of Order $v$ ) Let $D_{v}=d_{0}, d_{1}$, $\ldots, d_{\ell-1}$ be a sequence of elements in $\mathbb{Z}_{v} \backslash\{0\}$ such that $d_{i} \neq d_{j}(\bmod v)$ when $i \neq j$. 
- If $v \equiv 0(\bmod 2)$, then $D_{v}$ is a cyclic difference sequence of order $v$ if and only if $d_{i} \neq v / 2$, for all $i \in\{0,1, \ldots, \ell-1\}$, and $2\left\{\left(\mathbb{Z}_{v} \backslash\{0\}\right) \backslash \pm\left\{D_{v}\right\}\right\}= \pm\left\{\sigma_{D_{v}}^{\prime}\right\}$.

- If $v \equiv 1(\bmod 2)$, then $D_{v}$ is a cyclic difference sequence of order $v$ if and only if $2\left\{\left(\mathbb{Z}_{v} \backslash\{0\}\right) \backslash \pm\left\{D_{v}\right\}\right\}= \pm\left\{\sigma_{D_{v}}^{\prime}\right\}$.

This definition is equivalent to saying that every non-zero element or its negative (but not both) in $\{1,2, \ldots,\lfloor v / 2\rfloor\}$ must either appear in $D_{v}$ or must appear in $\sigma_{D_{v}}^{\prime}$ twice. Note that when $v \equiv 0(\bmod 2)$, the difference $v / 2 \equiv-v / 2(\bmod v)$, thus, the difference $v / 2$ must appear in $\sigma_{D_{v}}^{\prime}$, and must appear exactly once. Given its properties, a cyclic difference sequence must be of length $(v-1) / 3$. To illustrate the concept, we give examples of each type of cyclic difference sequence. The sequence $D_{16}=1,4,2,3,7$ is a cyclic difference sequence of order 16 with $\sigma_{D_{16}}^{\prime}=5,6,5,-6,8$. The sequence $D_{25}=5,-6,7,-10,12,-8,11,-9$ is a cyclic difference sequence of order 25 with $\sigma_{D_{25}}^{\prime}=-1,1,-3,2,4,3,2,-4$.

Definition 4.1.2. (Difference Sequence of Order $v$ ) Let $D_{v}=d_{0}, d_{1}, \ldots, d_{\ell-1}$ be a sequence of elements in $\mathbb{Z}_{v} \backslash\{0\}$ such that $d_{i} \neq d_{j}(\bmod v)$ when $i \neq j$.

- If $v \equiv 0(\bmod 2)$, then $D_{v}$ is a difference sequence of order $v$ if and only if $d_{i} \neq v / 2$, for all $i \in\{0,1, \ldots, \ell-1\}$, and $2\left\{\left(\mathbb{Z}_{v} \backslash\{0\}\right) \backslash \pm\left\{D_{v}\right\}\right\} \cup \pm\left\{d_{\ell-1}\right\}=$ $\pm\left\{\sigma_{D_{v}}\right\}$

- If $v \equiv 1(\bmod 2)$, then $D_{v}$ is a difference sequence of order $v$ if and only if 


$$
2\left\{\left(\mathbb{Z}_{v} \backslash\{0\}\right) \backslash \pm\left\{D_{v}\right\}\right\} \cup \pm\left\{d_{\ell-1}\right\}= \pm\left\{\sigma_{D_{v}}\right\}
$$

This definition is equivalent to saying that every non-zero element or its negative (but not both) in $\{1,2, \ldots,\lfloor v / 2\rfloor\}$ must either appear in $D_{v}$ or must appear in $\sigma_{D_{v}}$ twice, except for the last element in $D_{v}$ which must appear exactly once in $D_{v}$ and exactly once in $\sigma_{D_{v}}$. In order to have these properties, a difference sequence must be of length $(v+1) / 3$. To illustrate the concept, we give examples of each type of difference sequence. The sequence $D_{14}=3,-1,-6,4,5$ is a difference sequence of order 14 with $\sigma_{D_{14}}=2,7,-2,-5$. Notice that 5 appears both in $D_{14}$ and in $\sigma_{D_{14}}$ (as -5 ), and that 7 appears exactly once in $\sigma_{D_{14}}$. The sequence $D_{11}=1,3,2,4$ is a difference sequence of order 11 with $\sigma_{D_{11}}=4,5,-5$.

Difference sequences and cyclic difference sequences may seem like artificial constructs, however, their properties reflect their intended use: cyclic and non-cyclic difference sequences can be used to construct the base blocks of twofold triple systems. We will describe the construction in a following paragraph; however, to motivate the definitions of difference sequences we note that the construction method will imply that if $D_{v}$ is cyclic, each entry of $D_{v}$ will appear as a difference in two base blocks, while each entry of $\sigma_{D_{v}}^{\prime}$ will appear once in a base block. If $D_{v}$ is not cyclic, each entry of $D_{v}$ will appear as a difference in two base blocks, except the last which will appear in one base block, and each entry of $\sigma_{D_{v}}$ will appear once in a base block.

Independently, Aldred, Bailey, McKay and Wanless [44] have defined a concept 
similar to a difference sequence. Given a positive integer $m$, they look for sequences of length $m-1$ containing elements of $\mathbb{Z}_{m} \backslash\{0\}$ such that the following properties hold: (1) the sum of each pair of consecutive elements in the sequence is distinct and non-zero, (2) the non-zero element missing from these sums is 1 if $m$ is odd and $m / 2+1$ if $m$ is even, and (3) the sum of the last and the first elements in the sequence is one. Aldred, Bailey, McKay and Wanless have also defined several variants of this sequence.

The existence of difference sequences is an interesting question in and of itself, however, we have developed the concept of a difference sequence because of its implication for designs. Given a cyclic difference sequence of order $v, D_{v}=d_{0}, d_{1}, \ldots, d_{\ell-1}$, define the non-cyclic sequence

$$
U_{D_{v}}^{\prime}=0, d_{0}, d_{0}+d_{1}, d_{0}+d_{1}+d_{2}, \ldots, \sum_{i=0}^{\ell-1} d_{i}, \sum_{i=0}^{\ell-1} d_{i}+d_{0}
$$

where each sum is taken modulo $v$. Similarly, given a difference sequence of order $v$, $D_{v}=d_{0}, d_{1}, \ldots, d_{\ell-1}$, define the non-cyclic sequence

$$
U_{D_{v}}=\infty, 0, d_{0}, d_{0}+d_{1}, d_{0}+d_{1}+d_{2}, \ldots, \sum_{i=0}^{\ell-1} d_{i},
$$

where each sum is taken modulo $v$.

Lemma 4.1.2. (i) The existence of a cyclic difference sequence of order $v$ implies the existence of a cyclic TTS $(v)$ and the existence of a partial Ustring representing its base blocks. 
(ii) The existence of a difference sequence of order $v-1$ implies the existence of a 1-rotational TTS $(v)$ and the existence of a partial Ustring representing its base blocks.

Proof. (i) Let $D_{v}=d_{0}, d_{1}, \ldots, d_{\ell-1}, \ell=(v-1) / 3$, be a cyclic difference sequence of order $v$. Construct the sequence

$$
U_{D_{v}}^{\prime}=0, d_{0}, d_{0}+d_{1}, d_{0}+d_{1}+d_{2}, \ldots, \sum_{i=0}^{\ell-1} d_{i}, \sum_{i=0}^{\ell-1} d_{i}+d_{0}
$$

where each sum is taken modulo $v$. We prove this sequence is a non-cyclic partial Ustring representing the base blocks of a cyclic TTS $(v)$. The elements of $U_{D_{v}}^{\prime}$ are points in $\mathbb{Z}_{v}$ and every set of three consecutive elements represents a block, therefore, $U_{D_{v}}^{\prime}$ represents an ordering of $\ell$ blocks. Each of the differences in $D_{v}$ appears in two of the blocks represented by $U_{D_{v}}^{\prime}$ and each of the differences in $\sigma_{D_{v}}^{\prime}$ appears in one of the blocks represented by $U_{D_{v}}^{\prime}$. To see this, consider a portion of $U_{D_{v}}^{\prime}$, say

$$
\sum_{i=0}^{j-1} d_{i}, \sum_{i=0}^{j} d_{i}, \sum_{i=0}^{j+1} d_{i}, \sum_{i=0}^{j+2} d_{i}
$$

This sequence represents the blocks

$$
\left\{\sum_{i=0}^{j-1} d_{i}, \sum_{i=0}^{j} d_{i}, \sum_{i=0}^{j+1} d_{i}\right\} \text { and }\left\{\sum_{i=0}^{j} d_{i}, \sum_{i=0}^{j+1} d_{i}, \sum_{i=0}^{j+2} d_{i}\right\} .
$$

The differences $d_{j}, d_{j+1}$ and $d_{j}+d_{j+1}$ appear in the first block and the differences $d_{j+1}, d_{j+2}$ and $d_{j+1}+d_{j+2}$ appear in the second block. In general, each element of $D_{v}$ is a difference in two consecutive blocks, whereas the sum of two consecutive elements 
in $D_{v}$ is a difference in one block. The structure of $D_{v}$ and $\sigma_{D_{v}}^{\prime}$ implies that the set of blocks represented by $U_{D_{v}}^{\prime}$ has each difference in $\mathbb{Z}_{v} \backslash\{0\}$ appearing exactly twice in a block. Therefore, the partial Ustring $U_{D_{v}}^{\prime}$ represents the base blocks of a cyclic $\operatorname{TTS}(v)$

(ii) Let $D_{v-1}=d_{0}, d_{1}, \ldots, d_{\ell-1}, \ell=v / 3$, be a difference sequence of order $v-1$.

Construct the sequence

$$
U_{D_{v-1}}=\infty, 0, d_{0}, d_{0}+d_{1}, d_{0}+d_{1}+d_{2}, \ldots, \sum_{i=0}^{\ell-1} d_{i},
$$

where each sum is taken modulo $v-1$. We prove the sequence $U_{D_{v-1}}$ is a non-cyclic partial Ustring representing the base blocks of a 1-rotational TTS $(v)$. Each of the differences in $D_{v-1}$, except $d_{\ell-1}$, appears in two of the blocks represented by $U_{D_{v-1}}$ and each of the differences in $\sigma_{D_{v-1}}$ appears in one of the blocks represented by $U_{D_{v-1}}$. By definition, $d_{\ell-1}$ appears in $\sigma_{D_{v-1}}$ exactly once and in $D_{v-1}$ exactly once, therefore, each difference in $\mathbb{Z}_{v-1} \backslash\{0\}$ appears exactly twice in a block. The partial Ustring $U_{D_{\boldsymbol{v} \sim 1}}$ represents the base blocks of a 1-rotational TTS $(v)$.

The cyclic difference sequence of order $25, D_{25}=5,-6,7,-10,12,-8,11,-9$, induces the non-cyclic partial Ustring $U_{D_{25}}^{\prime}=0,5,-1,6,-4,8,0,11,2,7$. This partial Ustring represents the list of blocks $\{0,5,24\},\{5,24,6\},\{24,6,21\},\{6,21,8\}$, $\{21,8,0\},\{8,0,11\},\{0,11,2\}$ and $\{11,2,7\}$. These are the base blocks of a cyclic TTS(25) as each difference in $\mathbb{Z}_{25} \backslash\{0\}$ occurs twice. The difference sequence $D_{14}=$ $3,-1,-6,4,5$ of order 14 induces a non-cyclic partial Ustring $U_{D_{14}}=\infty, 0,3,2,-4,0,5$. 
This partial Ustring represents the list of blocks $\{\infty, 0,3\},\{0,3,2\},\{3,2,10\},\{2,10,0\}$, and $\{10,0,5\}$. These are the base blocks of a 1-rotational TTS(15) as each difference in $\mathbb{Z}_{14} \backslash\{0\}$ occurs twice.

We refer again to the work of Aldred, Bailey, McKay and Wanless [44]. Their sequence (described just before Lemma 4.1.2) can be developed in a similar way to that described in the proof of Lemma 4.1.2 to obtain a circular sequence of length $m(m-1)$ on $m$ elements in which every ordered pair of distinct elements occurs exactly once as neighbours and exactly once with a single item between them.

Lemma 4.1.3. Let $D_{v}$ be a cyclic difference sequence of order $v$. If the sum of the elements in $D_{v}$ is coprime to $v$, then there exists a Ucycle of rank three for the blocks of the cyclic TTS(v) induced by $D_{v}$.

Proof. Given $D_{v}$, a cyclic difference sequence of order $v$, and $U_{D_{v}}^{\prime}$, the non-cyclic partial Ustring induced by $D_{v}$, create $v-1$ other non-cyclic partial Ustrings by developing $U_{D_{v}}^{\prime}$ through $\mathbb{Z}_{v} \backslash\{0\}$. As $U_{D_{v}}^{\prime}$ represents the base blocks of a $\operatorname{TTS}(v)$, this collection of partial Ustrings represents all the blocks of the $\operatorname{TTS}(v)$. It remains to join these sequences together to form a Ucycle for the design. The first block represented by $U_{D_{v}}^{\prime}$ is $\left\{0, d_{0}, d_{0}+d_{1}\right\}$ and the last block represented by $U_{D_{v}}^{\prime}$ is $\left\{\sum_{i=0}^{\ell-2} d_{i}, \sum_{i=0}^{\ell-1} d_{i}, \sum_{i=0}^{\ell-1} d_{i}+\right.$ $\left.d_{0}\right\}$, with points appearing in this order in $U_{D_{v}}^{\prime}$. In order to maintain the Ucycle representation across joins, we must join the end of $U_{D_{v}}^{\prime}$ to another partial Ustring which must begin with $\sum_{i=0}^{\ell-1} d_{i}, \sum_{i=0}^{\ell-1} d_{i}+d_{0}, x$, for some $x \in \mathbb{Z}_{v}$. Notice that the sequence 
$U_{D_{v}}^{\prime}+\sum_{i=0}^{\ell-1} d_{i}$ has first block $\left\{\sum_{i=0}^{\ell-1} d_{i}, \sum_{i=0}^{\ell-1} d_{i}+d_{0}, \sum_{i=0}^{\ell-1} d_{i}+d_{0}+d_{1}\right\}$. We can join the end of $U_{D_{v}}^{\prime}$ to the beginning of $U_{D_{v}}^{\prime}+\sum_{i=0}^{\ell-1} d_{i}$, then we can join the end of $U_{D_{v}}^{\prime}+\sum_{i=0}^{\ell-1} d_{i}$ to the beginning of $U_{D_{v}}^{\prime}+\left(2 \cdot \sum_{i=0}^{\ell-1} d_{i}\right)$, and so on. Note that in joining partial Ustrings together we identify their two shared points. As long as $\sum_{i=0}^{\ell-1} d_{i}$ is coprime to $v$ (the number of partial Ustrings in question) all partial Ustrings can be joined to form a single Ucycle for the design.

Recall that the cyclic difference sequence $D_{25}=5,-6,7,-10,12,-8,11,-9$ induces the non-cyclic partial Ustring $U_{D_{25}}^{\prime}=0,5,-1,6,-4,8,0,11,2,7$. The sum of the entries in $D_{25}$ is $2(\bmod 25)$. Since 2 and 25 are coprime, we can create a Ucycle for the TTS(25) having base blocks represented by $U_{D_{25}}^{\prime}$. In the sequence below, the pairs of bold entries indicate the join of partial Ustrings (the bold points appear both at the end of one partial Ustring and at the beginning of another partial Ustring).

$$
\begin{gathered}
\mathbf{0}, \mathbf{5},-1,6,-4,8,0,11, \mathbf{2}, \mathbf{7}, 1,8,-2,10,2,-12 \\
\mathbf{4}, \mathbf{9}, 3,10,0,12,4,-10, \mathbf{6}, \mathbf{1 1}, 5,12,2,-11,6,-8, \\
\mathbf{8},-\mathbf{1 2}, 7,-11,4,-9,8,-6, \mathbf{1 0},-\mathbf{1 0}, 9,-9,6,-7,10,-4 \\
\mathbf{1 2}, \mathbf{- 8}, \mathbf{1 1},-7,8,-5,12,-2,-\mathbf{1 1},-\mathbf{6},-12,-5,10,-3,-11,0 \\
\mathbf{- 9}, \mathbf{- 4},-10,-3,12,-1,-9,2,-\mathbf{7},-\mathbf{2},-8,-1,-11,1,-7,4 \\
\mathbf{- 5}, \mathbf{0},-6,1,-9,3,-5,6,-\mathbf{3}, \mathbf{2},-4,3,-7,5,-3,8 \\
\mathbf{- 1}, \mathbf{4},-2,5,-5,7,-1,10, \mathbf{1}, \mathbf{6}, 0,7,-3,9,1,12 \\
\mathbf{3}, \mathbf{8}, 2,9,-1,11,3,-11, \mathbf{5}, \mathbf{1 0}, 4,11,1,-12,5,-9
\end{gathered}
$$




$$
\begin{aligned}
& \mathbf{7}, \mathbf{1 2}, 6,-12,3,-10,7,-7, \mathbf{9}, \mathbf{- 1 1}, 8,-10,5,-8,9,-5 \\
& \mathbf{1 1}, \mathbf{- 9}, 10,-8,7,-6,11,-3, \mathbf{- 1 2}, \mathbf{- 7}, 12,-6,9,-4,-12,-1 \text {, } \\
& \mathbf{- 1 0},-\mathbf{5},-11,-4,11,-2,-10,1,-\mathbf{8},-\mathbf{3},-11,-2,-12,0,-8,3 \text {, } \\
& \mathbf{- 6}, \mathbf{- 1},-7,0,-10,2,-6,5,-4, \mathbf{1},-7,2,-8,4,-4,7 \text {, } \\
& \mathbf{- 2}, \mathbf{3},-3,4,-6,6,-2,9, \mathbf{0}, \mathbf{5},-3,6,-4,8,0,11 \text {, } \\
& \mathbf{2}, 7,1,7,-2,10,2,-11, \mathbf{4}, \mathbf{9}, 1,10,0,12,4,-10 \\
& \mathbf{6}, \mathbf{1 1}, 5,11,2,-11,6,-7, \mathbf{8},-\mathbf{1 2}, 5,-11,4,-9,8,-6 \text {, } \\
& \mathbf{1 0}, \mathbf{- 1 0}, 9,-10,6,-7,10,-3, \mathbf{1 2},-\mathbf{8}, 9,-7,8,-5,12,-2 \text {, } \\
& \mathbf{- 1 1}, \mathbf{- 6},-12,-6,10,-3,-11,1, \mathbf{- 9}, \mathbf{- 4},-12,-3,12,-1,-9,2 \text {, } \\
& \mathbf{- 7}, \mathbf{- 2},-8,-2,-11,1,-7,5,-\mathbf{5}, \mathbf{0},-8,1,-9,-5,6 \text {, } \\
& \mathbf{- 3}, \mathbf{2},-4,2,-7,5,-3,9,-\mathbf{1}, \mathbf{4},-4,5,-5,-1,10, \\
& \mathbf{1}, \mathbf{6}, 0,6,-3,9,1,-12, \mathbf{3}, \mathbf{8}, 0,9,-1,3,-11 \\
& \mathbf{5}, \mathbf{1 0}, 4,10,1,-12,5,-8, \mathbf{7}, \mathbf{1 2}, 4,-12,3,7,-7 \text {, } \\
& \mathbf{9}, \mathbf{- 1 1}, 8,-11,5,-8,9,-4, \mathbf{1 1}, \mathbf{- 9}, 8,-8,7,11,-3 \text {, } \\
& \mathbf{- 1 2},-\mathbf{7}, 12,-7,9,-4,-12,0,-\mathbf{1 0},-\mathbf{5}, 12,-4,11,-10,1 \text {, } \\
& \mathbf{- 8}, \mathbf{- 3},-9,-3,-12,0,-8,4, \mathbf{- 6}, \mathbf{- 1},-9,0,-12,-6,5 \text {, } \\
& \mathbf{- 4}, \mathbf{1},-5,1,-8,4,-4,8, \mathbf{- 2}, \mathbf{3},-5,4,-8,-2,9 \text {, } \\
& \mathbf{0}, \mathbf{5},-1, \cdots
\end{aligned}
$$

Theorem 4.1.4. (i) For each $v \equiv 1(\bmod 12)$, with $v \not \equiv 0(\bmod 5)$, there exists $a$ $T T S(v)$ that admits a Ucycle of rank three. 
(ii) For each $v \equiv 4(\bmod 12)$, with $v \not \equiv 0(\bmod 5)$, there exists a TTS(v) that admits a Ucycle of rank three.

(iii) For each $v \equiv 7(\bmod 12)$ there exists a TTS $(v)$ that admits a Ucycle of rank three.

Proof. To prove the existence of Ucycles for $\operatorname{TTS}(v)$ s with $v \equiv 1,4,7(\bmod 12)$, we present a cyclic difference sequence for each equivalence class and show that Lemma 4.1.3 holds.

(i) Suppose $v \equiv 1(\bmod 12)$ and let $v=12 m+1$. Define

$$
\begin{gathered}
\alpha=-(3 m+1), 3 m,-(3 m+2), 3 m-1, \ldots, 2 m+1,-(4 m+1) \text { and } \\
\beta=4 m+2,6 m,-(4 m+3), 6 m-1,-(4 m+4), 6 m-2, \ldots \\
\ldots,-(5 m-1), 5 m+3,-5 m, 5 m+2,5 m+1
\end{gathered}
$$

and set $D_{v}=\alpha, \beta$. Notice that in each sequence alternate elements form subsequences in which entries progress by plus one or minus one, except the first and last entries of $\beta$. Ignoring signs, the union of the elements in $\alpha$ and $\beta$ is the set $\{2 m+1, \ldots, 6 m\} \subset \mathbb{Z}_{v}$. As $\sigma_{\alpha}=-1,-2, \ldots,-2 m$ and $\sigma_{\beta}=2 m-1,2 m-3,2 m-4, \ldots, 3,2,-(2 m-2)$, it remains to determine if the differences 1 and $2 m$ (or the negation of these values) appear once more as sums of two consecutive elements in $D_{v}$. At the join of the sequences $\alpha$ and $\beta$ we have $\ldots,-4 m-1,4 m+2, \ldots$, which yields the sum 1 . Because 
$D_{v}$ is a cyclic sequence we also consider the sum of the last entry in $\beta$ and the first entry in $\alpha$. This sum is $5 m+1-3 m-1=2 m$.

It remains to determine when the sum of the entries in $D_{v}$ is coprime to $v$. The sum of the entries in $D_{v}$ can be obtained by taking the sum of the first and every alternate value in $\sigma_{D_{v}}$, plus the last element in $D_{v}$ if it is not taken into account by these sums. From $\sigma_{\alpha}$ we have the sum of the odd values from -1 to $-(2 m-1)$. The sum of differences across the join of $\alpha$ and $\beta$ is one. Finally, taking the second element in $\sigma_{\beta}$ and every alternate element, yields a sum of the positive odd values from 3 to $2 m-3$ plus $10 m+3$ (the sum of $5 m+2$ and $5 m+1$ ). Therefore, the sum of the entries in $D_{v}$ is $8 m+4$. Since $\operatorname{gcd}(8 m+4,12 m+1)=\operatorname{gcd}(10,4 m-3)$, we conclude that $\operatorname{gcd}(8 m+4,12 m+1)=1$ for all $m \not \equiv 2(\bmod 5)$. Therefore, the sum of the elements in $D_{v}$ is coprime to $v$ for all $v \not \equiv 0(\bmod 5)$, which implies that there exists a cyclic $\operatorname{TTS}(v)$ admitting a Ucycle of rank three for $v \equiv 1,13,37,49(\bmod 60)$.

(ii) Suppose $v \equiv 4(\bmod 12)$ and let $v=12 m+4$. Define

$$
\begin{gathered}
\alpha=4 m+1,-(6 m+1), 4 m+2,-6 m, \ldots,-(5 m+2), 5 m+1, \text { and } \\
\beta=-(3 m+1), 3 m,-(3 m+2), 3 m-1, \ldots,-4 m, 2 m+1,
\end{gathered}
$$

and set $D_{v}=\alpha, \beta$. Ignoring signs, the union of the elements in $\alpha$ and $\beta$ is the set $\{2 m+1, \ldots, 6 m+1\} \subset \mathbb{Z}_{v}$. As $\sigma_{\alpha}=-2 m,-(2 m-1), \ldots,-1$ and $\sigma_{\beta}=-1,-2, \ldots$ $\ldots,-(2 m-1)$, it remains to determine if the differences $2 m$ (or $-2 m)$ and $6 m+2$ (which is equal to $v / 2$, so must appear only once as a sum) appear again as sums 
of two consecutive elements in $D_{v}$. At the join of the sequences $\alpha$ and $\beta$ we have $\ldots, 5 m+1,-3 m-1, \ldots$, which yields the sum $2 m$. The sum of the last entry in $\beta$ and first entry in $\alpha$ is $2 m+1+4 m+1=6 m+2$. Finally, we determine when the sum of the entries in $D_{v}$ is coprime to $v$. The sum of the entries in $\alpha$ is

$$
-\left(\sum_{j=1}^{m}(2 j)\right)+5 m+1
$$

and the sum of the entries in $\beta$ is

$$
-\left(\sum_{j=1}^{m}(2 j-1)\right)
$$

Therefore, the sum of the entries in $D_{v}$ is

$$
-\left(\sum_{j=1}^{m} j\right)+5 m+1=-\left(\frac{m(m+1)}{2}\right)+5 m+1=\frac{-m^{2}+9 m+2}{2} .
$$

The $\operatorname{gcd}\left(12 m+4,\left(-m^{2}+9 m+2\right) / 2\right)=1$ for all $v \not \equiv 0(\bmod 5)$, which implies that there exists a cyclic $\operatorname{TTS}(v)$ admitting a Ucycle of rank three for $v \equiv 4,16,28,52$ $(\bmod 60)$.

(iii) Suppose $v \equiv 7(\bmod 12)$ and let $v=12 m+7$. Define

$$
\begin{gathered}
\alpha=4 m+2,-(6 m+3), 4 m+3,-(6 m+2), \ldots, 5 m+2,-(5 m+3), \text { and } \\
\beta=3 m+2,-(3 m+1), 3 m+3,-3 m, \ldots, 4 m+1,-(2 m+2),
\end{gathered}
$$

and set $D_{v}=\alpha, \beta$. Ignoring signs, the union of the elements in $\alpha$ and $\beta$ is the set $\{2 m+2, \ldots, 6 m+3\} \subset \mathbb{Z}_{v}$. As $\sigma_{\alpha}=-(2 m+1),-2 m, \ldots,-1$ and $\sigma_{\beta}=1,2, \ldots$ 
$\ldots, 2 m-1$, it remains to determine if the differences $2 m+1$ and $2 m$ (or the negative of these values) appear again as sums of two consecutive elements in $D_{v}$. Again, consider the joins of $\alpha$ and $\beta$. At the end of $\alpha$ and the beginning of $\beta$ we have $\ldots,-5 m-3,3 m+2, \ldots$ which yields a sum of $-(2 m+1)$. At the end of $\beta$ and the beginning of $\alpha$ we have $\ldots,-2 m-2,4 m+2$ which yields a sum of $2 m$.

It remains to determine when the sum of the entries in $D_{v}$ is coprime to $v$. The sum of the entries in $\alpha$ is the sum of the odd numbers from -1 to $-(2 m+1)$, while the sum of the entries in $\beta$ is the sum of the odd numbers from 1 to $2 m-1$. Therefore, the sum of the entries in $D_{v}$ is $-(2 m+1)$. Since $\operatorname{gcd}(2 m+1,12 m+7)=\operatorname{gcd}(2 m+1,1)$, we conclude that $v$ is always coprime to $2 m+1$. There exists a cyclic TTS $(v)$ admitting a Ucycle of rank three for $v \equiv 7(\bmod 12)$.

When $v \equiv 0,3,6,9(\bmod 12)$, it is impossible to create Ucycles of rank three for the blocks of a $\operatorname{TTS}(v)$ as three does not divide $v-1$ (see Lemma 3.2.3 on page 82), therefore, for $\operatorname{TTS}(v) \mathrm{s}$ of these orders we investigate the existence of 2-intersecting Gray cycles.

Lemma 4.1.5. Let $v$ be odd and let $D_{v-1}=d_{0}, d_{1}, \ldots, d_{\ell-1}$ be a difference sequence of order $v-1$. If (1) $d_{0}$ is coprime to $v-1$, (2) $d_{\ell-1}=-(2 n+1) d_{0}$, and (3) $d_{\ell-2}=2(2 n+1) d_{0}$, for some $n \in \mathbb{Z}_{v-1}$ that is coprime to $(v-1) / 2$, then there exists a 2-intersecting Gray cycle for the blocks of the 1-rotational TTS $(v)$ induced by $D_{v-1}$ 
Proof. Suppose $D_{v-1}=d_{0}, d_{1}, \ldots, d_{\ell-1}$ is a difference sequence of order $v-1$ with $d_{0}$ coprime to $v-1, d_{\ell-2}=2(2 n+1) d_{0}$ and $d_{\ell-1}=-(2 n+1) d_{0}$, where $n \in \mathbb{Z}_{v-1}$ is coprime to $(v-1) / 2$. Recall (from Lemma 4.1.2 (ii)) that $U_{D_{v-1}}=\infty, 0, d_{0}, d_{0}+$ $d_{1}, d_{0}+d_{1}+d_{2}, \ldots, \sum_{i=0}^{\ell-1} d_{i}$, with addition performed modulo $v-1$, is the non-cyclic partial Ustring induced by $D_{v-1}$. Create $v-2$ other non-cyclic partial Ustrings by developing $U_{D_{v-1}}$ through $\mathbb{Z}_{v-1} \backslash\{0\}$. As $U_{D_{v-1}}$ represents the base blocks of a 1rotational $\operatorname{TTS}(v)$, this collection of partial Ustrings represents all the blocks of the 1-rotational TTS $(v)$. Let $L_{D_{v-1}}$ be the list of blocks represented by $U_{D_{v-1}}$ :

$$
\left[\left\{\infty, 0, d_{0}\right\},\left\{0, d_{0}, d_{0}+d_{1}\right\},\left\{d_{0}, d_{0}+d_{1}, d_{0}+d_{1}+d_{2}\right\}, \ldots,\left\{\sum_{i=0}^{\ell-3} d_{i}, \sum_{i=0}^{\ell-2} d_{i}, \sum_{i=0}^{\ell-1} d_{i}\right\}\right] .
$$

Because we are constructing a Gray cycle, we will now work with $L_{D_{v-1}}$ and $L_{D_{v-1}, x}$, $x \in\{1,2, \ldots, v-2\}$, where $L_{D_{v-1}, x}$ denotes the sequence of blocks represented by the Ustring $U_{D_{v-1}}+x$. It remains to join these lists of blocks together to form a 2-intersecting Gray cycle; we must ensure that the blocks on either side of a join share two points.

The first block in $L_{D_{v-1}}$ is $\left\{\infty, 0, d_{0}\right\}$. This block shares two points with the first block of $L_{D_{v-1}, d_{0}}$ which is $\left\{\infty, d_{0}, 2 d_{0}\right\}$. Write $L_{D_{v-1}}$ in reverse order and join it to $L_{D_{v-1}, d_{0}}$. Write $L_{D_{v-1}, 2 d_{0}}$ in reverse order and join it to $L_{D_{v-1}, 3 d_{0}}$, and so on. As $v-1$ is even, the sequences $L_{D_{v-1}, x}, x \in\{0,1, \ldots, v-1\}$, can all be paired in this manner because $d_{0}$ is coprime to $v-1$. There are now $(v-1) / 2$ sequences of the form $\overline{L_{D_{v-1}, 2 m d_{0}}}, L_{D_{v-1},(2 m+1) d_{0}}, m \in\{0,1, \ldots,(v-1) / 2\}$, where $\overline{L_{D_{v-1}}}$ indicates that 
the sequence $L_{D_{v-1}}$ is written in reverse order. Each of these sequences starts with a block of the form $\left\{x, x+2(2 n+1) d_{0}, x+(2 n+1) d_{0}\right\}$ and ends with a block of the form $\left\{x+d_{0}, x+2(2 n+1) d_{0}+d_{0}, x+(2 n+1) d_{0}+d_{0}\right\}, x \in \mathbb{Z}_{v-1}$. We must now join these new sequences together such that the blocks on either side of each join share two points. Consider the block $B=\left\{x, x+2(2 n+1) d_{0}, x+(2 n+1) d_{0}\right\}$ which appears at the beginning of the sequence $\overline{L_{D_{v-1}}}, L_{D_{v-1}, d_{0}}$. The difference $(2 n+1) d_{0}$ appears twice in $B$, therefore, $B+(2 n+1) d_{0}$ has two points in common with $B$. The block $B+(2 n+1) d_{0}=\left\{x+(2 n+1) d_{0}, x+3(2 n+1) d_{0}, x+\right.$ $\left.2(2 n+1) d_{0}\right\}$ appears as the last block of $L_{D_{v-1},(2 n+1) d_{0}}$. Since $\operatorname{gcd}(n,(v-1) / 2)=1$, we can join all sequences together to form a 2-intersecting Gray cycle as follows: $\left[\overline{L_{D_{v-1}}}, L_{D_{v-1}, d_{0}}, \overline{L_{D_{v-1},-2 n d_{0}}}, L_{D_{v-1},-(2 n-1) d_{0}}, \ldots, \overline{L_{D_{v-1}, 2 n d_{0}}}, L_{D_{v-1},(2 n+1) d_{0}}\right]$.

Consider the difference sequence $D_{14}=3,-1,-6,4,5$ of order 14 . The noncyclic partial Ustring induced by this difference sequence is $U_{D_{14}}=\infty, 0,3,2,-4,0,5$ and this string represents the blocks $L_{D_{14}}=\{\infty, 0,3\},\{0,3,2\},\{3,2,10\},\{2,10,0\}$, $\{10,0,5\}$. Creating the intermediate sequences, we join $\overline{L_{D_{14}}}$ to $L_{D_{14}, 3}$ which yields $\{10,0,5\},\{2,10,0\},\{3,2,10\},\{0,3,2\},\{\infty, 0,3\},\{\infty, 3,6\},\{3,6,5\},\{6,5,13\},\{5$, $13,3\},\{13,3,8\}$. We also join $\overline{L_{D_{14}, 6}}$ to $L_{D_{14}, 9}$ and $\overline{L_{D_{14}, 12}}$ to $L_{D_{14}, 1}$, and so on. Finally, we write $\overline{L_{D_{14}}}, L_{D_{14}, 3}, \overline{L_{D_{14}, 8}}, L_{D_{14}, 11}, \ldots \overline{L_{D_{14}, 6}}, L_{D_{14}, 9}$. The complete 2-intersecting Gray cycle for the 1-rotational TTS(15) induced by $D_{14}$ is given in Table 12 .

Note that Lemma 4.1 .5 may only be applied when $v \equiv 3,9(\bmod 12)$ as $v-1$ 


$\begin{array}{llll}\{10,0,5\} & \{12,2,7\} & \{0,4,9\} & \{2,6,11\} \\ \{2,10,0\} & \{4,12,2\} & \{6,0,4\} & \{8,2,6\} \\ \{3,2,10\} & \{5,4,12\} & \{7,6,0\} & \{9,8,2\} \\ \{0,3,2\} & \{2,5,4\} & \{4,7,6\} & \{6,9,8\} \\ \{\infty, 0,3\} & \{\infty, 2,5\} & \{\infty, 4,7\} & \{\infty, 6,9\} \\ \{\infty, 3,6\} & \{\infty, 5,8\} & \{\infty, 7,10\} & \{\infty, 9,12\} \\ \{3,6,5\} & \{5,8,7\} & \{7,10,9\} & \{9,12,11\} \\ \{6,5,13\} & \{8,7,1\} & \{10,9,3\} & \{12,11,5\} \\ \{5,13,3\} & \{7,1,5\} & \{9,3,7\} & \{11,5,9\} \\ \{13,3,8\} & \{1,5,10\} & \{3,7,12\} & \{5,9,0\} \\ \{4,8,13\} & \{6,10,1\} & \{8,12,3\} & \\ \{10,4,8\} & \{12,6,10\} & \{0,8,12\} & \\ \{11,10,4\} & \{13,12,6\} & \{1,0,8\} & \\ \{8,11,10\} & \{10,13,12\} & \{12,1,0\} & \\ \{\infty, 8,11\} & \{\infty, 10,13\} & \{\infty, 12,1\} & \\ \{\infty, 11,0\} & \{\infty, 13,2\} & \{\infty, 1,4\} & \\ \{11,0,12\} & \{13,2,1\} & \{1,4,3\} & \\ \{0,12,7\} & \{2,1,9\} & \{4,3,11\} & \\ \{12,7,11\} & \{1,9,13\} & \{3,11,1\} & \\ \{7,11,2\} & \{9,13,4\} & \{11,1,6\} & \end{array}$

Table 12: A 2-intersecting Gray cycle for the blocks of the 1-rotational TTS(15) with base blocks given by the non-cyclic partial Ustring $U_{D_{14}}=\infty, 0,3,2,-4,0,5$ (read column-wise)

must be even.

Theorem 4.1.6. For each $v \equiv 3(\bmod 12), v>3$, there exists a TTS $(v)$ that admits a 2-intersecting Gray cycle.

Proof. For each $v \equiv 3(\bmod 12), v>3$, we prove that there exists a 1-rotational $\operatorname{TTS}(v)$ admitting a 2-intersecting Gray cycle. As we are working with 1-rotational designs, all operations will be done modulo $v-1$. The proof is broken into four cases: $v \equiv 3,15,27,39(\bmod 48)$. For each case we give a difference sequence and show 
that the properties required by Lemma 4.1 .5 hold. In each case $D_{v-1}$ consists of six subsequences. We will refer to these subsequences as $\alpha_{1}, \alpha_{2}, \beta, \gamma_{1}, \gamma_{2}$, and $e$, and we set $D_{v-1}=\alpha_{1}, \alpha_{2}, \beta, \gamma_{2}, \gamma_{1}, e$. The sixth subsequence is a single element and in each case will represent itself. $D_{v-1}$ is of length $v / 3$. The length of $\alpha_{1}$ is the same as that of $\gamma_{1}$ and the length of $\alpha_{2}$ is the same as that of $\gamma_{2}$ (although these lengths differ for each case). We will see that the elements of $\gamma_{1}$ and $\gamma_{2}$ are related to those of $\alpha_{1}$ and $\alpha_{2}$ by the following rule. Let $\alpha_{1}=x_{0}, x_{1}, \ldots, x_{\ell-2}$ and $\gamma_{1}=y_{0}, y_{1}, \ldots, y_{\ell-2}$, then

$$
y_{\ell-i}=f\left(x_{i}\right)= \begin{cases}(v-1) / 2-x_{i} & \text { if } x_{i}>0 \\ -(v-1) / 2-x_{i} & \text { if } x_{i}<0\end{cases}
$$

The same rule holds for $\alpha_{2}$ and $\gamma_{2}$. As a result, $\sigma_{\gamma_{1}}$ is the same as $\bar{\sigma}_{\alpha_{1}}$ with signs reversed. Similarly $\sigma_{\gamma_{2}}$ is the same as $\bar{\sigma}_{\alpha_{2}}$ with signs reversed. In fact, equation (4.1.1) holds for the entire sequence $D_{v-1}$ without $e$. Recall that the length of $D_{v-1}$ must be $v / 3$. While it may not be immediately obvious from the representations, it is not difficult to confirm that the sequence consisting of $\alpha_{1}, \alpha_{2}, \beta, \gamma_{2}, \gamma_{1}$ has the property that the sum of the $i^{t h}$ element and the $(v / 3-1)-i^{t h}$ element is either $-(v-1) / 2$ or $(v-1) / 2$ with each pair of summands of the same sign.

When $v \equiv 3,27(\bmod 48)$, the first entry of $D_{v-1}$ is $(v+3) / 6$. Due to the property stated above, when $v \equiv 3,27(\bmod 48)$, the second last element of $D_{v-1}$ is $(v-3) / 3$ (since $(v+3) / 6+(v-3) / 3=(v-1) / 2)$. Similarly, when $v \equiv 15,39(\bmod 48)$, the first entry of $D_{v-1}$ is $-(v+3) / 6$ and the second last element of $D_{v-1}$ is $-(v-3) / 3$. 
Lemma 4.1.5 requires that the first entry of $D_{v-1}$ be coprime to $v-1$. To determine $\operatorname{gcd}((v+3) / 6, v-1)$, substitute $v=12 m+3$. Since $\operatorname{gcd}(2 m+1,12 m+2)=\operatorname{gcd}(2 m+$ $1,-4)=1$, we conclude that $v-1$ is always coprime to $(v+3) / 6$. Lemma 4.1 .5 also requires that there exist an $n$ such that $2(2 n+1) d_{0}=d_{v / 3-2}$ and $-(2 n+1) d_{0}=d_{v / 3-1}$, with all operations modulo $v-1$. It will be easy to confirm that these two equations hold once our sequences have been constructed.

To determine that an $n$ with the above properties is coprime to $(v-1) / 2$, notice that the summation property implies that $d_{0}+d_{v / 3-2}=d_{0}+2(2 n+1) d_{0} \equiv(v-1) / 2$ $(\bmod (v-1))$. Substituting $v=12 m+3$ we obtain $(4 n+3) d_{0} \equiv(12 m+2) / 2$ $(\bmod v-1)$. We can divide this equality by 2 and remove $d_{0}$ as $d_{0}$ is coprime to $v-1$, which yields $2 n \equiv 3 m-1(\bmod (v-1) / 2)$. As $(v-1) / 2=6 m+1$, we have $(3 m+1) 2 n \equiv(3 m+1)(3 m-1)(\bmod (6 m+1))$. Solving for $n$ we obtain $n \equiv 9 m^{2}-1$ $(\bmod (6 m+1))$. We will choose the minimal $n$ that satisfies this equation. Finally, we must show that $\operatorname{gcd}\left(9 m^{2}-1,6 m+1\right)=1$ in order to prove that $n$ is coprime to $(v-1) / 2$. It is clear that if $\operatorname{gcd}(2 x, y)=1$, then $\operatorname{gcd}(x, y)=1$. Using this property, determine $\operatorname{gcd}\left(2\left(9 m^{2}-1\right), 6 m+1\right)$. This statement is equivalent to $\operatorname{gcd}(3 m-1,3)$, which is clearly one. Therefore, $n$ is coprime to $(v-1) / 2$. For each case it remains to prove that the given $D_{v-1}$ is a difference sequence. 
Case 1 Suppose $v \equiv 3(\bmod 48)$ and let $v=48 m+3$. Define

$$
\begin{aligned}
\alpha_{1}= & 8 m+1,-(10 m+1), 10 m+2,-10 m, 10 m+3, \ldots, \\
& \ldots,-(9 m+2), 11 m+1, \\
\alpha_{2}= & -15 m, 13 m-1,-(15 m+1), 13 m-2, \ldots \\
& \ldots, 12 m+2,-(16 m-2), 12 m+1,-(16 m-1), \\
\beta= & 8 m-1,20 m+1,8 m-2,20 m, \ldots, 18 m+2,6 m-1, \ldots \\
& \quad \ldots, 4 m+1,16 m+3,4 m, 16 m+2, \\
\gamma_{1}= & 13 m,-(15 m-1), 13 m+1,-(15 m-2), \ldots, \\
& \quad \ldots, 14 m-2,-(14 m+1), 14 m-1,-14 m, 16 m, \\
\gamma_{2}= & -(8 m+2), 12 m,-(8 m+3), 12 m-1, \ldots, \\
& \quad \ldots, 11 m+3,-9 m, 11 m+2,-(9 m+1) .
\end{aligned}
$$

Set $D_{v-1}=\alpha_{1}, \alpha_{2}, \beta, \gamma_{2}, \gamma_{1}, 16 m+1$. Notice that the sequence $\alpha_{1}$ follows a clear sequential pattern, except for the first entry. Equation 4.1.1 implies that $\gamma_{1}$ also follows a sequential pattern, except for its last entry. Ignoring signs, together the sequences $\alpha_{1}, \alpha_{2}, \beta, \gamma_{1}$, and $\gamma_{2}$ contain $\{4 m, \ldots, 8 m-1\} \cup\{8 m+1, \ldots, 16 m\} \cup$ $\{16 m+2, \ldots, 20 m+1\} \subset \mathbb{Z}_{v-1}$. The sums of pairs of consecutive elements in $\alpha_{1}$ is $\sigma_{\alpha_{1}}=-2 m, 1,2, \ldots, 2 m-1$ and the sums of pairs of consecutive elements in $\alpha_{2}$ is $\sigma_{\alpha_{2}}=-(2 m+1),-(2 m+2), \ldots,-(4 m-3),-(4 m-2)$. The sequence $\beta$ has sums of consecutive pairs of elements $\sigma_{\beta}=28 m, 28 m-1, \ldots, 24 m+1, \ldots, 20 m+3,20 m+2$, 
which is equivalent to $-(20 m+2),-(20 m+3), \ldots, 24 m+1, \ldots, 20 m+3,20 m+2$. Because the sums of consecutive pairs of elements in $\gamma_{1}$ are the same as those in $\alpha_{1}$ with signs reversed (similarly for $\gamma_{2}$ and $\alpha_{2}$ ), the sums of pairs of consecutive elements in the sequences $\alpha_{1}, \alpha_{2}, \beta, \gamma_{1}$ and $\gamma_{2}$ cover $\pm\{\{1, \ldots, 4 m-2\} \cup\{20 m+$ $2, \ldots, 24 m\}\} \cup\{24 m+1\}$. The four sums of two consecutive elements appearing at the joins of sequences $\alpha_{1}, \alpha_{2}, \beta, \gamma_{2}$ and $\gamma_{1}$ are $\pm\left(4 m-1\right.$ ) (between $\alpha_{1}$ and $\alpha_{2}$ and between $\gamma_{1}$ and $\gamma_{2}$ ) and $\pm 8 m$ (between $\alpha_{2}$ and $\beta$ and between $\beta$ and $\gamma_{2}$ ). Finally, $16 m+1$ appears once as the last element in $D_{v-1}$ and once in negative form as the sum across the join of $\gamma_{1}$ and $16 m+1($ because $32 m+1 \equiv-(16 m+1)(\bmod 48 m+2))$.

Case 2 Suppose $v \equiv 15(\bmod 48)$ and let $v=48 m+15$. A 2-intersecting Gray 
cycle for a 1-rotational $\operatorname{TTS}(15)$ is given in Table 12 (page 123). For $v>15$, define

$$
\begin{aligned}
\alpha_{1}= & -(8 m+3), 12 m+2,-(8 m+4), 12 m+1, \ldots, \\
& \ldots, 11 m+4,-(9 m+2), \\
\alpha_{2}= & 9 m+3,-(11 m+3), 9 m+4,-(11 m+2), \ldots \\
& \ldots, 10 m+2,-(10 m+4), 14 m+4,-(12 m+3), \\
\beta= & 4 m+1,16 m+6,4 m+2,16 m+7, \ldots, 6 m+1,18 m+6, \ldots \\
& \ldots, 8 m, 20 m+5,8 m+1,20 m+6, \\
\gamma_{1}= & -(15 m+5), 13 m+3, \ldots, \\
& \ldots, 12 m+6,-(16 m+3), 12 m+5,-(16 m+4), \\
\gamma_{2}= & -(12 m+4), 10 m+3,-(14 m+3), 14 m+5, \ldots, \\
& \ldots,-(13 m+5), 15 m+3,-(13 m+4), 15 m+4 .
\end{aligned}
$$

Set $D_{v-1}=\alpha_{1}, \alpha_{2}, \beta, \gamma_{2}, \gamma_{1},-(16 m+5)$. Notice that the sequence $\alpha_{2}$ follows a clear sequential pattern, except for the last two entries. Equation 4.1 .1 implies that $\gamma_{2}$ also follows a sequential pattern, except for its first two entries. Ignoring signs, together the sequences $\alpha_{1}, \alpha_{2}, \beta, \gamma_{1}$, and $\gamma_{2}$ contain $\{4 m+1, \ldots, 8 m+1\} \cup\{8 m+3, \ldots, 16 m+$ $4\} \cup\{16 m+6, \ldots, 20 m+6\} \subset \mathbb{Z}_{v-1}$. The sums of pairs of consecutive elements in $\alpha_{1}$ is $\sigma_{\alpha_{1}}=4 m-1,4 m-2, \ldots, 2 m+2$ and the sums of pairs of consecutive elements in $\alpha_{2}$ is $\sigma_{\alpha_{2}}=-2 m,-(2 m-1), \ldots,-2,4 m, 2 m+1$. The sequence $\beta$ has sums of consecutive pairs of elements $\sigma_{\beta}=20 m+7,20 m+8, \ldots, 24 m+7, \ldots, 28 m+6,28 m+7$, which 
is equivalent to $20 m+7,20 m+8, \ldots, 24 m+6,24 m+7,-(24 m+6), \ldots,-(20 m+$ $8),-(20 m+7)$. Because the sums of consecutive pairs of elements in $\gamma_{1}$ are the same as those in $\alpha_{1}$ with signs reversed (similarly for $\gamma_{2}$ and $\alpha_{2}$ ), the sums of pairs of consecutive elements in the sequences $\alpha_{1}, \alpha_{2}, \beta, \gamma_{1}$ and $\gamma_{2}$ cover $\pm\{\{2, \ldots, 4 m\} \cup$ $\{20 m+7, \ldots, 24 m+6\}\} \cup\{24 m+7\}$. The four sums of two consecutive elements appearing at the joins of sequences $\alpha_{1}, \alpha_{2}, \beta, \gamma_{2}$ and $\gamma_{1}$ are \pm 1 (between $\alpha_{1}$ and $\alpha_{2}$ and between $\gamma_{1}$ and $\left.\gamma_{2}\right)$ and $\pm(8 m+2)$ (between $\alpha_{2}$ and $\beta$ and between $\beta$ and $\gamma_{2}$ ). Finally, $-(16 m+5)$ appears once as the last element in $D_{v-1}$ and once in positive form as the sum across the join of $\gamma_{1}$ and $-(16 m+5)$. 
Case 3 Suppose $v \equiv 27(\bmod 48)$ and let $v=48 m+27$. Define

$$
\begin{aligned}
\alpha_{1}= & 8 m+5,-(10 m+6), 10 m+7,-(10 m+5), 10 m+8, \ldots \\
& \ldots, 11 m+6,-(9 m+6), \\
\alpha_{2}= & 13 m+6,-(15 m+8), 13 m+5,-(15 m+9), \ldots \\
& \ldots,-(16 m+5), 12 m+8,-(16 m+6), 12 m+7,-(8 m+6), \\
\beta= & 16 m+10,4 m+2,16 m+11,4 m+3, \ldots, 6 m+2,18 m+11, \ldots \\
& \ldots, 20 m+10,8 m+2,20 m+11,8 m+3, \\
\gamma_{1}= & -(15 m+7), 13 m+7, \ldots, \\
& \ldots, 14 m+5,-(14 m+8), 14 m+6,-(14 m+7), 16 m+8, \\
\gamma_{2}= & -(16 m+7), 12 m+6,-(8 m+7), 12 m+5,-(8 m+8), \ldots \\
& \ldots,-(9 m+5), 11 m+7 .
\end{aligned}
$$

Set $D_{v-1}=\alpha_{1}, \alpha_{2}, \beta, \gamma_{2}, \gamma_{1}, 16 m+9$. Notice that the sequence $\alpha_{1}$ follows a clear sequential pattern, except for the first entry. As a result, the sequence $\gamma_{1}$ follows a clear sequential pattern, except for the last entry. Also, $\alpha_{2}$ follows a clear sequential progression, except for its last entry, which implies that $\gamma_{2}$ follows a clear sequential progression, except for its first entry. Ignoring signs, together the sequences $\alpha_{1}, \alpha_{2}, \beta, \gamma_{1}$, and $\gamma_{2}$ contain $\{4 m+2, \ldots, 8 m+3\} \cup\{8 m+5, \ldots, 16 m+$ $8\} \cup\{16 m+10, \ldots, 20 m+11\} \subset \mathbb{Z}_{v-1}$. The sums of pairs of consecutive elements in $\alpha_{1}$ is $\sigma_{\alpha_{1}}=-(2 m+1), 1,2, \ldots, 2 m$ and the sums of pairs of consecutive 
elements in $\alpha_{2}$ is $\sigma_{\alpha_{2}}=-(2 m+2),-(2 m+3), \ldots,-(4 m-2),-(4 m-1), 4 m+1$. The sequence $\beta$ has sums of consecutive pairs of elements $\sigma_{\beta}=20 m+12,20 m+$ $13, \ldots, 24 m+12,24 m+13,24 m+14, \ldots, 28 m+13,28 m+14$, which is equivalent to $20 m+12,20 m+13, \ldots, 24 m+12,24 m+13,-(24 m+12), \ldots,-(20 m+13),-(20 m+12)$. Because the sums of consecutive pairs of elements in $\gamma_{1}$ are the same as those in $\alpha_{1}$ with signs reversed (similarly for $\gamma_{2}$ and $\alpha_{2}$ ), the sums of pairs of consecutive elements in the sequences $\alpha_{1}, \alpha_{2}, \beta, \gamma_{1}$ and $\gamma_{2}$ cover $\pm\{\{1, \ldots,-(4 m-1)\} \cup\{4 m+1\} \cup$ $\{20 m+12, \ldots, 24 m+12\}\} \cup\{24 m+13\}$. The four sums of two consecutive elements appearing at the joins of sequences $\alpha_{1}, \alpha_{2}, \beta, \gamma_{2}$ and $\gamma_{1}$ are $\pm 4 m$ (between $\alpha_{1}$ and $\alpha_{2}$ and between $\gamma_{1}$ and $\left.\gamma_{2}\right)$ and $\pm(8 m+4)$ (between $\alpha_{2}$ and $\beta$ and between $\beta$ and $\gamma_{2}$ ). Finally, $16 m+9$ appears once as the last element in $D_{v-1}$ and once in negative form as the sum across the join of $\gamma_{1}$ and $16 m+9$. 
Case 4 Suppose $v \equiv 39(\bmod 48)$ and let $v=48 m+39$. Define

$$
\begin{aligned}
\alpha_{1}= & -(8 m+7), 10 m+8,-(10 m+9), 10 m+7,-(10 m+10), \ldots \\
& \ldots,-(11 m+8), 9 m+8, \\
\alpha_{2}= & -(13 m+10), 15 m+12,-(13 m+9), 15 m+13, \ldots \\
& \ldots, 16 m+11,-(12 m+10), \\
\beta= & 20 m+16,8 m+5,20 m+15,8 m+4, \ldots, 18 m+15,6 m+4, \ldots \\
& \ldots, 16 m+15,4 m+4,16 m+14,4 m+3, \\
\gamma_{1}= & 15 m+11,-(13 m+11), \ldots, \\
& \ldots,-(14 m+9), 14 m+12,-(14 m+10), 14 m+11,-(16 m+12), \\
\gamma_{2}= & -(12 m+9), 8 m+8, \ldots, \\
& \ldots, 9 m+6,-(11 m+10), 9 m+7,-(11 m+9) .
\end{aligned}
$$

Set $D_{v-1}=\alpha_{1}, \alpha_{2}, \beta, \gamma_{2}, \gamma_{1},-(16 m+13)$. Notice that the sequence $\alpha_{1}$ follows a clear sequential pattern, except for the first entry. As a result, the sequence $\gamma_{1}$ follows a clear sequential pattern, except for the last entry. Ignoring signs, together the sequences $\alpha_{1}, \alpha_{2}, \beta, \gamma_{1}$, and $\gamma_{2}$ contain $\{4 m+3, \ldots, 8 m+5\} \cup\{8 m+7, \ldots, 16 m+$ $12\} \cup\{16 m+14, \ldots, 20 m+16\}$. The sums of pairs of consecutive elements in $\alpha_{1}$ is $\sigma_{\alpha_{1}}=2 m+1,-1,-2, \ldots,-2 m$ and the sums of pairs of consecutive elements in $\alpha_{2}$ is $\sigma_{\alpha_{2}}=2 m+2,2 m+3, \ldots, 4 m+1$. The sequence $\beta$ has sums of consecutive pairs of elements $\sigma_{\beta}=28 m+21,28 m+20, \ldots, 24 m+20,24 m+19,24 m+18, \ldots, 20 m+$ 
$18,20 m+17$, which is equivalent to $-(20 m+17),-(20 m+18), \ldots,-(24 m+18), 24 m+$ $19,24 m+18, \ldots, 20 m+18,20 m+17$. Because the sums of consecutive pairs of elements in $\gamma_{1}$ are the same as those in $\alpha_{1}$ with signs reversed (similarly for $\gamma_{2}$ and $\alpha_{2}$ ), the sums of pairs of consecutive elements in the sequences $\alpha_{1}, \alpha_{2}, \beta, \gamma_{1}$ and $\gamma_{2}$ cover $\pm\{\{1, \ldots, 4 m+1\} \cup\{20 m+17, \ldots, 24 m+18\}\} \cup\{24 m+19\}$. The four sums of two consecutive elements appearing at the joins of sequences $\alpha_{1}, \alpha_{2}, \beta, \gamma_{2}$ and $\gamma_{1}$ are $\pm(4 m+2)$ (between $\alpha_{1}$ and $\alpha_{2}$ and between $\gamma_{1}$ and $\left.\gamma_{2}\right)$ and $\pm(8 m+6)$ (between $\alpha_{2}$ and $\beta$ and between $\beta$ and $\left.\gamma_{2}\right)$. Finally, $-(16 m+13)$ appears once as the last element in $D_{v-1}$ and once in positive form as the sum across the join of $\gamma_{1}$ and $-(16 m+13)$.

Investigation into the existence of Ucycles of rank three for $v \equiv 10(\bmod 12)$ and 2-intersecting Gray codes for $v \equiv 0,6,9(\bmod 12)$ continues. We have constructed a 2-intersecting Gray cycle for a $\operatorname{TTS}(v)$ of each order $v \leq 200$ where $v \equiv 9(\bmod 12)$. For $v \equiv 0(\bmod 12)$, we must either deal with 1-rotational systems or cyclic systems containing short orbit base blocks, while for $v \equiv 6(\bmod 12)$ we must deal with 1 rotational systems. It will be necessary to determine a new method for joining partial Ustrings together to create 2-intersecting Gray codes in these two cases.

Conjecture 4.1.7. For each $v \equiv 0,6,9(\bmod 12)$, there exists a TTS $(v)$ that admits a 2-intersecting Gray code.

For each $v \equiv 10(\bmod 12)$, there exists a 1-rotational $\operatorname{TTS}(v)$, but these designs also contain a short orbit base block. While the same general methods are applicable, 
CHAPTER 4. GRAY CODES AND UNIVERSAL CYCLES FOR DESIGNS 134

some adjustment must be made to the definition of difference sequence to accommodate short orbit base blocks. Colbourn et al. have generated all TTS(10)s having no repeated blocks $([18])$. We obtained this listing and wrote a program to search for Ucycles of rank three for each of these designs. This search is not difficult to execute because once the first block and the order of appearance of elements in this block has been fixed, the entry of new blocks into the sequence is forced. We found that no simple TTS(10) admits a Ucycle of rank three for its blocks, and therefore, that no TTS(10) admits a Ucycle of rank three for its blocks. However, we conjecture that there exist higher order $\operatorname{TTS}(v) \mathrm{s}$ with $v \equiv 10(\bmod 12)$ that do admit rank three Ucycles.

Conjecture 4.1.8. For each $v \equiv 10(\bmod 12), v \geq 22$, there exists a TTS $(v)$ that admits a rank three Ucycle.

Finally, we believe it is possible to "fill in the gaps" in Theorem 4.1.4.

Conjecture 4.1.9. (i) For each $v \equiv 1(\bmod 12)$, with $v \equiv 0(\bmod 5)$, there exists a TTS $(v)$ that admits a Ucycle of rank three.

(ii) For each $v \equiv 4(\bmod 12)$, with $v \equiv 0(\bmod 5)$, there exists a TTS $(v)$ that admits a Ucycle of rank three.

Our results regarding the existence of 2-intersecting Gray cycles (here we think of the rank three Ucycles as 2-intersecting Gray cycles) for TTSs lead to the following 
two theorems.

Theorem 4.1.10. For each $v \equiv 1,3,4,7(\bmod 12)$, with exceptions as stated in Theorem 4.1.4, there exists a TTS $(v)$ that admits an $A_{3}$-ordering (Figure 1 on page 7).

Theorem 4.1.11. For each $v \equiv 1,3,4,7(\bmod 12)$, with exceptions as stated in Theorem 4.1.4, there exists a TTS $(v)$ that admits a $\left\{B_{6}, B_{7}\right\}$-ordering (Figure 18).

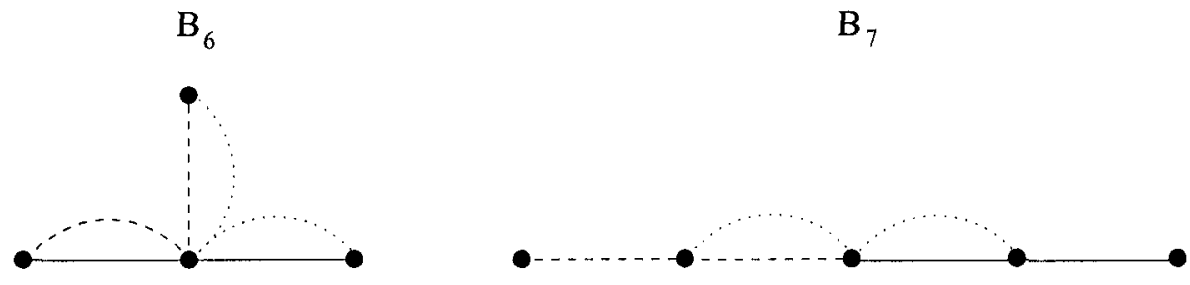

Figure 18: The configurations $B_{6}$ and $B_{7}$

\subsection{Gray Codes and Universal Cycles for BIBDs}

We have seen that there exist infinite families of twofold triple systems that admit 2-intersecting Gray cycles (see Section 4.1). What can be said about minimal change orderings for $(v, k, \lambda)$-BIBDs in general? When index is one and strength is two, each pair of blocks in a $(v, k, \lambda)$-BIBD intersect in at most one point. In this case, we can ask only for a weak minimal change property; consecutive blocks must share exactly one common element. The existence of such a minimal change ordering is equivalent to the existence of a Hamilton path or cycle in the 1-block intersection 
graph. The existence of Hamilton cycles in 1-block intersection graphs is entirely determined for $(v, K, 1)$-PBDs. Hare's result in [30] implies that if $\min (K) \geq 3$, a $(v, K, 1)-\mathrm{PBD}$ admits a minimal change ordering for its blocks. When the index is larger than one or the strength of the design is greater than two, stronger minimal change properties can be defined. Existence results in this area are scattered. M. Colbourn and Johnstone have discussed non-existence of 2-intersecting Gray codes for twofold triple systems [20], while we have shown that 2-intersecting Gray cycles exist for several infinite families of twofold triple systems (Theorems 4.1.4 and 4.1.6). Much work has been done regarding the existence of Gray codes and Ucycles for $k$ subsets of $n$-sets $[7,23,24,39,59]$ and these results can be applied to designs of the form $\left(v, k,\left(\begin{array}{c}v-2 \\ k-2\end{array}\right)\right)$-BIBD.

We turn now to the existence of Ucycles for $(v, k, \lambda)$-BIBDs. While block size is variable, the strength of all $(v, k, \lambda)$-BIBDs is two, therefore, we investigate the existence of rank two Ucycles. In this section we focus on cyclic designs. As discussed in Section 3.2 and recalled above, when $\lambda=1$, the best possible arrangement for the blocks of a $(v, k, \lambda)$-BIBD is to have consecutive blocks intersecting in one element. In this case a pair of points represents a unique block, therefore, a rank two Ucycle contains all the information necessary to reconstruct the design. However, when $\lambda>1$, each pair of points appears in $\lambda$ blocks of the design and so a pair of consecutive points in a rank two Ucycle does not uniquely determine a block of the BIBD. In order 
to recover the blocks of a $(v, K, \lambda)$-BIBD with $\lambda>1$ from a rank two Ucycle, we will need to provide some additional information.

Recall from Section 2.1.4 that, given a design $S=(V, \mathcal{B})$, the base block coloured pair adjacency graph $G_{b a s e}^{V}$ is a multigraph with vertex set $V$ and an edge of colour $B$ between vertices $v_{1}$ and $v_{2}$ if the difference $v_{2}-v_{1}$ appears in the base block $B$. A Ucycle of rank two describes a tour in $G_{\text {base }}^{V}$. Each vertex of $G_{\text {base }}^{V}$ may not be in this tour. More importantly, this tour will include edge colour $B$ exactly $|D e v(B)|$ times. Given a Ucycle of rank two for a cyclic $(v, k, \lambda)$-BIBD and the colour of the edge induced in $G_{\text {base }}^{V}$ by each pair of consecutive elements in the Ucycle, we can completely recover the blocks of the design from the Ucycle. The edge colour is the minimal amount of additional information required to recover these blocks.

While we are most interested in determining the existence of Ucycles for block designs, the existence of such an ordering has other implications. A design admitting a Ucycle of rank two is an Eulerian design. A design is Eulerian if and only if the block intersection graph of the design is Hamiltonian and there exists a Hamilton cycle in the block intersection graph which does not pass through any 3-claws in the design. Note that this definition of Eulerian is not the same as one used in the context of hypergraphs [3].

At this time, we are only able to prove that Ucycles of rank two exist for certain $(v, k, \lambda)$-BIBDs; however, we suspect that almost all designs admit Ucycles of rank 
two. There are, however, some exceptions. The $(6,\{2,3\}, 1)$-PBD consisting of the blocks of the Fano plane with one point removed does not admit a Ucycle of rank two. This design is not a member of the following infinite family of PBDs which also do not admit Ucycles of rank two.

Theorem 4.2.1. Given $v \in \mathbb{N}$ and any partition of $v=\sum_{i=1}^{\ell} v_{i}$ such that $v_{1} \geq 3$ and $v_{i} \geq 2$, for all $i \in\{2,3, \ldots, \ell\}$, there exists a $\left(v, 2 \cup\left\{v_{i}: 1 \leq i \leq \ell\right\}, 1\right)-P B D$ that does not admit a rank two Ucycle.

Proof. Define a pairwise balanced design on $v$ points as follows. Partition the points into $\ell$ disjoint classes $G_{1}, G_{2}, \ldots, G_{\ell}$ (represented in Figure 19) such that $\left|G_{i}\right|=v_{i}$, for all $i \in\{1,2, \ldots, \ell\}$. Let the blocks of the design be the classes of the partition and all pairs of points not in the same partition. That is, the design has point set $V=\cup_{i=1}^{\ell} G_{i}$ and blocks $\mathcal{B}=\left\{G_{1}, G_{2}, \ldots, G_{\ell}\right\} \cup\left\{\{x, y\}: x, y \in V, x \in G_{i}, y \in G_{j}, i \neq j\right\}$. In a

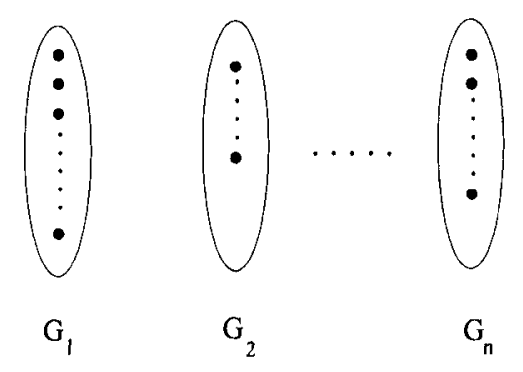

Figure 19: Classes used in constructing a $(v,\{2\} \cup K, 1)-\mathrm{PBD}$, with $\min K \geq 3$, that does not admit a Ucycle

rank two Ucycle for this design, each class $G_{i}$ will be represented by a pair of points from the class. The other blocks in the design are pairs of points and therefore will 
represent themselves. Each of these pairs must appear in a rank two Ucycle, thus, the existence of a rank two Ucycle is equivalent to the existence of an Euler cycle in the graph having the points of $\cup_{i=1}^{\ell} G_{i}$ as vertices and edges the pairs representing each block. Denote this graph by $H$. For an Euler cycle to exist, every vertex must have even degree. Consider a point $p$ in $G_{1}$. The number of edges incident to $p$ in $H$ is $\left|G_{2}\right|+\left|G_{3}\right|+\ldots+\left|G_{\ell}\right|$, unless $p$ is one of the two elements that represent $G_{1}$, in which case $p$ has one additional edge incident. Since $\left|G_{1}\right| \geq 3, G_{1}$ contains at least one point of each type. Therefore, both types of vertex appear in $H$, implying that $H$ cannot have an Euler cycle.

As blocks of size two appear to create problems in the construction of rank two Ucycles, we conjecture the following. The terms 'sufficient' and 'non-regular' will be defined later in this section.

Conjecture 4.2.2. Every $(v, K, \lambda)-P B D$, with $\min K \geq 3$ and a sufficient number of non-regular base blocks, admits a Ucycle of rank two.

At this time we can prove this conjecture only for certain cyclic designs, however, we see this as significant progress towards a general result. In Section 6.1, we discuss some of the difficulties in proving this conjecture in full generality and detail our progress in this area. In this section, we will prove that an infinite family of cyclic BIBDs admit Ucycles of rank two.

In a Ucycle of rank two, each consecutive pair of points in the sequence represents a 
block. Furthermore, this pair of points must appear in the block in question. That is, if $\{x, y\}$ represents block $B$, then $\{x, y\} \in B$. This means that the alphabet of a rank two Ucycle for a $\operatorname{BIBD}, S=(V, \mathcal{B})$, is $V^{\prime} \subseteq V$. Note that if the blocks of a design are of size greater than two, there is more than one way to represent each block. Throughout this section, we will be working with the points appearing in a given base block and the differences in that base block. Suppose $B_{d}=\left\{0, d_{1}, d_{2}, \ldots, d_{(k-1)}\right\}$ is a base block of a $(v, k, \lambda)$-BIBD. We will assume that the zero point appears in each base block and we will let $d_{0}=0$. The points in the base block $B_{d}$ are $d_{i}$, $i \in\{0,1, \ldots, k-1\}$, while the differences in $B_{d}$ are $\pm\left(d_{i}-d_{j}\right), i, j \in\{0,1, \ldots, k-1\}$, $i \neq j$. Whenever $d_{i}-d_{j}$ is a difference in a base block, $d_{j}-d_{i}$ is also a difference in the same base block, thus, we often talk about the differences $d_{i}-d_{j}$ with $i>j$. That is, we are interested in the $\left(\begin{array}{l}k \\ 2\end{array}\right)$ unordered pairs of points in the base block. Any block in $\operatorname{Dev}\left(B_{d}\right)$ can be chosen as the base block used to generate this set of blocks; however, many blocks in $\operatorname{Dev}\left(B_{d}\right)$ do not contain the point zero and hence are not suitable as base blocks under our assumption that base blocks contain the point zero. We will often wish to work with a representative of $\operatorname{Dev}\left(B_{d}\right)$ that has a given difference also appearing as one of the points in the base block. In this case, simply shift the base block $B_{d}$, by adding a fixed value to every entry in $B_{d}$, to obtain a base block containing the desired difference as a point. For example, if $d_{t}$ is a difference in $B_{d}=\left\{0, d_{1}, \ldots, d_{(k-1)}\right\}$ with $d_{t}=d_{i}-d_{j}$, then $d_{t}$ appears (as a point) in $-d_{j}+B_{d}$ 
(note that zero is also a point in this new expression of the base block).

While a Ucycle of rank two is a sequence of points in which consecutive pairs represent blocks, it helps to visualize a Ucycle as a cycle in the graph theoretic sense. That is, each point of the Ucycle is a vertex and there is an edge between each consecutive pair of points representing the block induced by the endpoints. To save space we will often write a Ucycle as a sequence of points, however to illustrate some concepts we will write a Ucycle as a cycle. When edges of this cycle are coloured to represent the base block from which the block represented is developed, this is a subgraph of the base block coloured pair adjacency graph for the design. In almost all cases, taking a consecutive pair of points in a Ucycle and knowing the colour of the edge connecting these two points uniquely determines the block represented. However, it is possible that two different blocks developed from the same base block are represented by the same pair. In this case we simply designate one occurrence of the pair to represent one block and the other occurrence of the pair to represent the other block. This ensures that every block of the design appears exactly once in the rank two Ucycle for the design.

Throughout this section, given a cyclic $(v, k, \lambda)$-BIBD, let $n_{1}$ denote the number of full orbit base blocks in the BIBD, let $n_{2}$ denote the number of non-regular short orbit base blocks in the BIBD, and let $n_{3}$ denote the number of regular short orbit base blocks in the BIBD. Also, $\phi(x), x \in \mathbb{Z}^{+}$, will denote the Euler totient function 
CHAPTER 4. GRAY CODES AND UNIVERSAL CYCLES FOR DESIGNS 142

which counts the number of positive integers less than or equal to $x$ that are coprime to $x$.

Theorem 4.2.3. Let $S$ be a cyclic $(v, k, \lambda)-B I B D$ with $k \geq 3$. If

$$
n_{2} \leq\left\lceil\frac{\lambda \phi(v)}{k(k-1)}\right\rceil-4 \quad \text { and } \quad n_{1}+n_{2} \geq\left\lfloor\frac{n_{3}}{2}\right\rfloor+3
$$

then $S$ admits a Ucycle of rank two.

Before proving Theorem 4.2.3, it is necessary to establish several auxiliary results. These results will provide the tools for a constructive proof of the existence of Ucycles of rank two for cyclic $(v, k, \lambda)$-BIBDs having the properties specified in Theorem 4.2.3. The process of determining a rank two Ucycle for a cyclic $(v, k, \lambda)$-BIBD begins by concentrating on a single base block. Given a base block and the blocks developed from it, we determine a rank two Ucycle for these blocks. A cyclic sequence of points that has the properties of a rank two Ucycle and represents some, but not all, blocks of a design will be called a partial Ucycle of rank two. There are some base blocks for which it is not possible to construct a rank two partial Ucycle. We deal with these blocks later when we join partial Ucycles representing the blocks developed from individual base blocks together to form a rank two Ucycle for all blocks of the design. Throughout the construction process, we will be careful to avoid creating an ordering of blocks which cannot be represented as a rank two Ucycle. In particular, we wish to avoid creating an ordering in which three consecutive blocks intersect in 
exactly one point and where this is the only intersection point for each of the two consecutive pairs of blocks from the triple. This is equivalent to saying we must avoid the claw configuration on three consecutive blocks. For triple systems, the 3-claw is the configuration denoted $B_{3}$ (see Figure 2 on page 8 ). For blocks of size $k$, the 3 -claw is the $(3 k-2,3)$-configuration in which one point appears in all three lines (as distinct from the other $(3 k-2,3)$-configuration which is the 3-path). An ordering which avoids the 3-claw will be said to have Property $\Pi$. For the remainder of this section we will assume the Ucycles referred to are of rank two.

Lemma 4.2.4. Let $S$ be a cyclic $(v, k, \lambda)-B I B D, k \geq 3$, and let $B_{d}=\left\{0, d_{1}, \ldots, d_{(k-1)}\right\}$ be a full orbit base block of $S$. If $\operatorname{gcd}\left(d_{1}, v\right)=1$, then there exists a partial Ucycle containing the blocks of Dev( $\left.B_{d}\right)$. We call this a length-v partial Ucycle.

Proof. Let $S$ be a cyclic $(v, k, \lambda)$-BIBD, $k \geq 3$, let $B_{d}=\left\{0, d_{1}, \ldots, d_{(k-1)}\right\}$ be a full orbit base block of $S$ and suppose $d_{1}$ is coprime to $v$. The cyclic sequence $U\left(B_{d}, d_{1}\right)=0, d_{1}, 2 d_{1}, \ldots,(v-1) d_{1}$, with multiplication performed modulo $v$, contains $v$ unique elements and thus $v$ unique consecutive pairs of points. Each consecutive pair of points represents a distinct block of $\operatorname{Dev}\left(B_{d}\right):\left\{x d_{1},(x+1) d_{1}\right\}$ represents $x d_{1}+B_{d}, x \in \mathbb{Z}_{v}$. Furthermore, the blocks represented by consecutive pairs of points have property $\Pi$. To see this, consider four consecutive points in $U\left(B_{d}, d_{1}\right)$ : $(a-1) d_{1}, a d_{1},(a+1) d_{1},(a+2) d_{1}$, where $a \in \mathbb{Z}_{v}$. These points represent the blocks 
$(a-1) d_{1}+B_{d}, a d_{1}+B_{d}$, and $(a+1) d_{1}+B_{d}$. Written out in full, these blocks are

$$
\begin{gathered}
\left\{(a-1) d_{1}, a d_{1},(a-1) d_{1}+d_{2}, \ldots,(a-1) d_{1}+d_{(k-1)}\right\}, \\
\left\{a d_{1},(a+1) d_{1}, a d_{1}+d_{2}, \ldots, a d_{1}+d_{(k-1)}\right\}, \text { and } \\
\left\{(a+1) d_{1},(a+2) d_{1},(a+1) d_{1}+d_{2}, \ldots,(a+1) d_{1}+d_{(k-1)}\right\}
\end{gathered}
$$

It is clear that the first two blocks intersect in $a d_{1}$ and the second two intersect in $(a+1) d_{1}$. These two (consecutive) intersection points are not the same because $d_{1} \neq 0$.

Notice that consecutive blocks represented by the length- $v$ partial Ucycle constructed in the proof of Lemma 4.2.4 may intersect in more than one point. While the number of points in the intersection of two consecutive blocks is forced when we look at Ucycles of rank $k$ for $(v, k, \lambda)$-BIBDs, in Ucycles of rank two we require only that each pair of consecutive blocks intersect in at least one point.

Suppose we are looking at a cyclic STS(15) generated by the base blocks $\{0,5,10\}$, $\{0,1,4\}$, and $\{0,2,8\}$. Note that the base block $\{0,5,10\}$ is a regular short orbit base block and contains no difference coprime to $v$, therefore, Lemma 4.2 .4 cannot be applied to it. However, the base block $\{0,1,4\}$ contains the difference 1 which is clearly coprime to 15 . The cycle $U=0,1,2,3,4,5,6,7,8,9,10,11,12,13,14$ is a length-15 partial Ucycle representing the ordering of $\operatorname{Dev}(\{0,1,4\})$ given in Table 13. Note that the cycle $U=0,4,8,12,1,5,9,13,2,6,10,14,3,7,11$ is also a Ucycle for 
this set of blocks.

$$
\begin{array}{lll}
\{0,1,4\} & \{5,6,9\} & \{10,11,14\} \\
\{1,2,5\} & \{6,7,10\} & \{11,12,0\} \\
\{2,3,6\} & \{7,8,11\} & \{12,13,1\} \\
\{3,4,7\} & \{8,9,12\} & \{13,14,2\} \\
\{4,5,8\} & \{9,10,13\} & \{14,0,3\}
\end{array}
$$

Table 13: The blocks of $\operatorname{Dev}(\{0,1,4\})$ in the order (read column-wise) given by the length-15 partial Ucycle $U=0,1,2,3,4,5,6,7,8,9,10,11,12,13,14$

Lemma 4.2 .4 can be applied using any of the differences appearing in $B_{d}$ as long as the difference is coprime to $v$. If $B_{d}$ is a full orbit base block and no difference in $B_{d}$ is coprime to $v$, we apply the following lemma.

Lemma 4.2.5. Let $S$ be a cyclic $(v, k, \lambda)-B I B D, k \geq 3$, and let $B_{d}=\left\{0, d_{1}, \ldots, d_{(k-1)}\right\}$ be a full orbit base block of $S$. If $\operatorname{gcd}\left(d_{1}, v\right)>1$, then there exists a collection of partial Ucycles (at least two) which considered together include each of the blocks of Dev $\left(B_{d}\right)$ exactly once. If the partial Ucycles are each of length $\ell$ (they will all be the same length) there will be $v / \ell$ partial Ucycles in the collection. We call this a collection

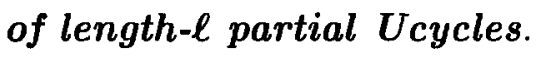

Proof. Let $S=(V, \mathcal{B})$ be a $(v, k, \lambda)$-BIBD, $k \geq 3$, let $B_{d}=\left\{0, d_{1}, \ldots, d_{(k-1)}\right\}$ be a full orbit base block of $S$ and suppose $\operatorname{gcd}\left(d_{1}, v\right)>1$. Let $c=\operatorname{lcm}\left(v, d_{1}\right)$. There exist $x, \ell \in$ $\mathbb{Z}$ such that $x v=c$ and $\ell d_{1}=c$. The cyclic sequence $U_{0}=0, d_{1}, 2 d_{1}, \ldots,(\ell-1) d_{1}$ contains $\ell$ unique elements of $V$ and thus $\ell$ unique pairs of consecutive points. Each 
consecutive pair of points represents a distinct block of $\operatorname{Dev}\left(B_{d}\right):\left\{x d_{1},(x+1) d_{1}\right\}$ represents $x d_{1}+B_{d}, x \in \mathbb{Z}_{\ell}$. For each $i \in\{1,2, \ldots,((v / \ell)-1)\}$, define $U_{i}$ to be the sequence obtained by taking each element of $U_{0}$ and adding $i$ to it. Consider the elements in $U_{0}$ as a subgroup of the group $\left(\mathbb{Z}_{v},+\right)$. The elements in each $U_{i}$ form a coset of $U_{0}$ in $\mathbb{Z}_{v}$, therefore, each element of $\mathbb{Z}_{v}$ appears exactly once in the collection $U\left(B_{d}, d_{1}\right)=\left\{U_{0}, U_{1}, \ldots, U_{(v / \ell)-1}\right\}$. The pair $\left\{x d_{1}+i,(x+1) d_{1}+i\right\}$ (on $\left.U_{i}\right)$ represents the block $\left(x d_{1}+i\right)+B_{d}$, so each pair of consecutive points in these cycles represents a unique block of $\operatorname{Dev}\left(B_{d}\right)$ and every block of $\operatorname{Dev}\left(B_{d}\right)$ is represented exactly once by the collection $U\left(B_{d}, d_{1}\right)$. The blocks represented by consecutive pairs of points in each $U_{i}, 0 \leq i \leq((v / \ell)-1)$, have property $\Pi$. This follows from the same reasoning as in the proof of Lemma 4.2.4.

Suppose we are looking at a $\operatorname{TTS}(24)$ that has base block $\{0,3,12\}$, among others. The differences in this base block are $3,9,12,12,15,21$, therefore, each difference has a greatest common divisor with 24 of three or greater. Select 3 as the difference with which to create the collection of partial Ucycles representing $\operatorname{Dev}(\{0,3,12\})$. As $\operatorname{lcm}(3,24)=24$, the blocks of $\operatorname{Dev}(\{0,3,12\})$ can be represented by three partial Ucycles, each of length eight. The partial Ucycles $U_{0}=0,3,6,9,12,15,18,21$, $U_{1}=1,4,7,10,13,16,19,22$, and $U_{2}=2,5,8,11,14,17,20,23$ represent the ordering of blocks given in Table 14. 


\begin{tabular}{cll}
$U_{0}$ & \multicolumn{1}{c}{$U_{1}$} & \multicolumn{1}{c}{$U_{2}$} \\
$\{0,3,12\}$ & $\{1,4,13\}$ & $\{2,5,14\}$ \\
$\{3,6,15\}$ & $\{4,7,16\}$ & $\{5,8,17\}$ \\
$\{6,9,18\}$ & $\{7,10,19\}$ & $\{8,11,20\}$ \\
$\{9,12,21\}$ & $\{10,13,22\}$ & $\{11,14,23\}$ \\
$\{12,15,0\}$ & $\{13,16,1\}$ & $\{14,17,2\}$ \\
$\{15,18,3\}$ & $\{16,19,4\}$ & $\{17,20,5\}$ \\
$\{18,21,6\}$ & $\{19,22,7\}$ & $\{20,23,8\}$ \\
$\{21,0,9\}$ & $\{22,1,10\}$ & $\{23,2,11\}$
\end{tabular}

Table 14: The blocks of $\operatorname{Dev}(\{0,3,12\})$ in the order given by the collection of length- 8 partial Ucycles $U_{0}=0,3,6,9,12,15,18,21, U_{1}=1,4,7,10,13,16,19,22$, and $U_{2}=$ $2,5,8,11,14,17,20,23$

Note that when a base block $B_{d}$ contains the difference $v / 2$ and this difference is used to generate the collection of partial Ucycles representing the blocks of $\operatorname{Dev}\left(B_{d}\right)$, the collection will consist of $v / 2$ length-2 partial Ucycles: $U_{0}=0, v / 2$, $U_{1}=1, v / 2+1, \ldots, U_{v / 2-1}=v / 2-1, v-1$. Note that each of these sequences represents two blocks. For example, if $B_{d}=\{0, v / 4, v / 2\}$ the sequence $U_{0}=0, v / 2$ represents the blocks $\{0, v / 4, v / 2\}$ and $\{v / 2,0,3 v / 4\}$.

In practice, given a full orbit base block, we will not know which difference was used to create the partial Ucycle (or Ucycles) representing the set of blocks generated by this base block, therefore, we refer to the partial Ucycle(s) representing the blocks developed from a single full orbit base block (i.e. generated using Lemmas 4.2 .4 and 4.2.5) as a collection of length $\ell$ partial Ucycles, where $\ell$ is between 2 and $v$.

Before delving further into the construction of Ucycles for cyclic BIBDs in general, 
we consider the existence of Ucycles for symmetric designs.

Corollary 4.2.6. Every cyclic symmetric $(v, k, \lambda)$-BIBD, $k \geq 3$, admits a Ucycle of rank two.

Proof. A cyclic symmetric design is generated by exactly one full orbit base block. As each difference in $\mathbb{Z}_{v}$ appears $\lambda$ times in this base block, Lemma 4.2 .4 can be applied using the difference 1. The full length partial Ucycle for the blocks developed from this base block is a Ucycle of rank two for the design.

The point-block incidence graph of a design $S=(V, \mathcal{B})$ is a bipartite graph $G=(V, \mathcal{B}, E)$. The subset denoted by $V$ contains vertices representing the points of the design and the subset denoted by $\mathcal{B}$ contains vertices representing the blocks of the design. The edge set of this graph is $E=\{(v, B): v \in B$, for $v \in V, B \in \mathcal{B}\}$. Define a $\mathcal{B}$-saturating cycle in $G$ to be a cycle that contains each point in $\mathcal{B}$ exactly once; points in $V$ may appear multiple times in this cycle or not at all. A $\mathcal{B}$-saturating cycle in $G$ is equivalent to determining a rank two Ucycle for the design represented. The point-block incidence graph of a symmetric $(v, k, \lambda)$-BIBD is regular of degree $k$ with $|V|=|\mathcal{B}|$. Using these two facts, we prove that the following family of symmetric designs admit Ucycles of rank two.

Lemma 4.2.7. Every symmetric $(v, k, \lambda)-B I B D$, with $k \geq 2$ and $v \leq 2 k-1$, admits a Ucycle of rank two. 
To prove Lemma 4.2.7 we require the following definition and Theorems 4.2 .8 and 4.2.9. Given a graph $G=(V, E)$ and a subset of vertices $W \subseteq V$, the subgraph of $G$ induced by the set $W$ is denoted $G[W]$. A supergraph of $G$, denoted $G^{\prime}=\left(V^{\prime}, E^{\prime}\right)$, is a graph with $V \subseteq V^{\prime}$ and $E \subseteq E^{\prime}$. The following theorem is due to Chvátal.

Theorem 4.2.8. (See [58]) Let $G$ be a simple graph on $n$ vertices with vertex degrees $d_{1} \leq \ldots \leq d_{n}$, where $n \geq 3$. If $i<n / 2$ implies that $d_{i}>i$ or $d_{n-i} \geq n-i$, then $G$ is Hamiltonian.

Theorem 4.2.9. (See [58]) Let $G=(X, Y, E)$ be a simple bipartite graph with $|X|=$ $|Y|=n / 2>1$. Let $G^{\prime}$ be the smallest supergraph of $G$ such that $G^{\prime}[Y]=K_{n / 2} . G$ is Hamiltonian if and only if $G^{\prime}$ is Hamiltonian.

Proof of Lemma 4.2.7. The point-block incidence graph of a symmetric $(v, k, \lambda)$-BIBD, whether cyclic or non-cyclic, is a bipartite graph $G=(V, \mathcal{B}, E)$ with $|V|=|\mathcal{B}|=v$, having all vertices of degree $k$. Create a supergraph of $G$ by adding to $G$ the edges that are required to make $G[\mathcal{B}]$ a complete graph. Denote the resulting graph $G^{\prime}$. Note that this new graph is no longer bipartite. To determine when $G^{\prime}$ admits a Hamilton cycle we appeal to Theorem 4.2.8. The degree sequence of $G^{\prime}$ is $k, \ldots, k, k+v-1, \ldots, k+v-1$, therefore, for $1 \leq i \leq k-1$, we have $d_{i}>i$. It is clear that $d_{i} \leq i$, for $k \leq i<2 v / 2$, therefore, in order for $G^{\prime}$ to admit a Hamilton cycle we require $d_{2 v-i} \geq 2 v-i$, for all $k \leq i<2 v / 2$. That is, we require $k+v-1 \geq 2 v-i$, for all $k \leq i<2 v / 2$, which simplifies to $v \leq k+i-1$, for all $k \leq i<2 v / 2$. Thus, 
when $v \leq 2 k-1, G^{\prime}$ admits a Hamilton cycle. Theorem 4.2 .9 implies that $G$, the point-block incidence graph of a symmetric $(v, k, \lambda)$-BIBD with $v \leq 2 k-1$, is Hamiltonian. Since a Hamilton cycle is certainly a $\mathcal{B}$-saturating cycle, such a design admits a Ucycle of rank two.

Lemmas 4.2.4 and 4.2.5 imply that a collection of partial Ucycles containing all blocks developed from a given base block can be created as long as the base block is not a short orbit base block. In fact, a collection of partial Ucycles containing all blocks developed from a given base block can be created as long as the base block is not a regular short orbit base block. We prove this statement in Lemmas 4.2 .10 and 4.2.11; however, we first discuss the forms of short orbit base blocks. A regular short orbit base block of a cyclic $(v, k, \lambda)$-BIBD has the form $\{0, v / k, 2 v / k, \ldots,(k-$ 1) $v / k\}$. Such a base block generates $v / k$ blocks which are pairwise disjoint. It is not possible to create a partial Ucycle of the form described in Lemmas 4.2.4 and 4.2.5 to represent the blocks developed from a regular short orbit base block unless the base block appears $k$ times in the design. In this unusual case, the sequence $U_{0}=0, v / k, 2 v / k, \ldots,(k-1) v / k$ is a partial Ucycle representing $k$ copies of the block $\{0, v / k, 2 v / k, \ldots,(k-1) v / k\}$. The collection of length- $(v / k)$ partial Ucycles formed by adding $i$ to the points of $U_{0}$, for each $i \in\{0,1, \ldots,(v-k) / k\}$, represents the blocks generated by $k$ copies of the regular short orbit base block. In general, this situation will not occur and the blocks developed from regular short orbit base blocks 
will need to be inserted into existing collections of partial Ucycles. This issue will be addressed in Lemmas 4.2.13 and 4.2.14.

Short orbit base blocks, whether regular or non-regular, have the following properties. The number of blocks generated by a short orbit base block is a divisor of $v$. The number of blocks generated by a short orbit base block (containing the zero point) must appear as a point in the base block. That is, if $B_{d}$ is a short orbit base block with $0 \in B_{d}$ and $n=\left|\operatorname{Dev}\left(B_{d}\right)\right|$, then $n \in B_{d}$. Furthermore, since $n+B_{d}=B_{d}$, the point $2 n$ must be in $B_{d}$. Continuing this argument implies that $B_{d}$ must contain the set of points $\{0, n, 2 n, \ldots,(x-1) n\}$, where $x$ is the minimal value in $\mathbb{Z}_{v}$ such that $x n \equiv 0(\bmod v)$. Non-regular short orbit base blocks must contain at least one difference that is not a multiple of $n$, therefore, each non-regular short orbit base block must contain at least the following set of points $\{0, n, 2 n, \ldots,(x-1) n, d, d+n, d+2 n, \ldots, d+(x-1) n\}, d \in \mathbb{Z}_{v}$, where $n \nmid d$. A base block containing exactly these points generates the fewest blocks of any non-regular short orbit base block. In this case $x=k / 2$ and since $n$ is the smallest number such that $x n \equiv 0(\bmod v), n=2 v / k$. Hence, each non-regular short orbit base block generates at least $2 v / k$ blocks. Note that every difference appearing in a short orbit base block appears at least twice.

To better understand short orbit base blocks, we present the possible short orbit base blocks for cyclic $(v, k, \lambda)$-BIBDs with small $k$. The only short orbit base block 
possible in a cyclic triple system is the regular short orbit base block $\{0, v / 3,2 v / 3\}$. Regular short orbit base blocks can only appear in $\operatorname{TS}(v, \lambda) \mathrm{s}$ with $v \equiv 3(\bmod 6)$. The short orbit base blocks associated with cyclic $(v, 4, \lambda)$-BIBDs have one general form. The regular short orbit base block $\{0, v / 4, v / 2,3 v / 4\}$ is a special case of the short orbit base block $\{0, v / 2, u, v / 2+u\}, 0<u<v / 2$. The only short orbit base block possible in a cyclic $(v, 5, \lambda)$-BIBD is the regular one. Finally, cyclic $(v, 6, \lambda)$-BIBDs may contain short orbit base blocks of the following two forms: $\{0, v / 2, u, u+v / 2, w, w+v / 2\}$, $u \neq w, 0<u, w<v / 2$ and $\{0, v / 3,2 v / 3, u, u+v / 3, u+2 v / 3\}, 0<u \leq v / 2$ (with the regular short orbit base block $\{0, v / 6,2 v / 6,3 v / 6,4 v / 6,5 v / 6\}$ a special case of each form).

The structure of non-regular short orbit base blocks means that at least one point in the base block is not a multiple of the number of blocks generated by the base block.

Lemma 4.2.10. Let $S$ be a cyclic $(v, k, \lambda)$-BIBD, $k \geq 3$, and let $B_{d}=\left\{0, d_{1}, \ldots\right.$, $\left.d_{(k-1)}\right\}$ be a non-regular short orbit base block of $S$. Let $d_{1}$ be a point that is not a multiple of $\left|\operatorname{Dev}\left(B_{d}\right)\right|$. If $\operatorname{gcd}\left(d_{1},\left|\operatorname{Dev}\left(B_{d}\right)\right|\right)=1$, then there exists a partial Ucycle containing the blocks of $\operatorname{Dev}\left(B_{d}\right)$.

Proof. Let $S$ be a $(v, k, \lambda)$-BIBD, $k \geq 3$, and let $B_{d}=\left\{0, d_{1}, \ldots, d_{(k-1)}\right\}$ be a nonregular short orbit base block of $S$. Let $n=\left|\operatorname{Dev}\left(B_{d}\right)\right|$ and let $m \in \mathbb{Z}_{v}$ be the smallest $m$ such that $m n \equiv 0(\bmod v)$. Suppose $d_{1}$ is coprime to $n$. The cyclic 
sequence $U\left(B_{d}, d_{1}\right)=0, d_{1}, 2 d_{1}, \ldots,(n-1) d_{1}$, with multiplication performed modulo $n$, contains $n$ unique elements and thus $n$ unique consecutive pairs of points. As in Lemma 4.2.4, each consecutive pair of points in $U\left(B_{d}, d_{1}\right)$ represents a distinct block of $\operatorname{Dev}\left(B_{d}\right)$; however, in this case we must show that $\left\{x d_{1}(\bmod n),(x+1) d_{1}\right.$ $(\bmod n)\}$ represents $x d_{1}(\bmod n)+B_{d}, x \in\{0,1, \ldots,(n-1)\}$. Let $y$ be the least residue of $x d_{1} \operatorname{modulo} n$. If $y+d_{1}(\bmod v)<n$, then the pair $\left\{y, y+d_{1}(\bmod n)\right\}$ is clearly in $y+B_{d}$. On the other hand, if $y+d_{1}(\bmod v)>n$, then we must show that $y+d_{1}(\bmod n)$ appears in $y+B_{d}$. Note that $y+d_{1} \equiv y+d_{1}-n(\bmod n)$ and that $y+d_{1}-n \equiv y+d_{1}+(m-1) n(\bmod v)$. The structure of non-regular short orbit base blocks implies that $y+\left(d_{1}+(m-1) n\right) \in y+B_{d}$ since the point $d_{1}+(m-1) n \in B_{d}$. Therefore, both points $y$ and $y+d_{1}(\bmod n)$ appear in $y+B_{d}$. Finally, the blocks represented by consecutive pairs of points have property $\Pi$ since $x d_{1}(\bmod n)+B_{d}$ intersects $(x+1) d_{1}(\bmod n)+B_{d}$ in $(x+1) d_{1}(\bmod n)$ and $(x+1) d_{1}(\bmod n)+B_{d}$ intersects $(x+2) d_{1}(\bmod n)+B_{d}$ in $(x+2) d_{1}(\bmod n)$. These two (consecutive) intersection points are not the same because $d_{1} \neq 0$.

Suppose $\{0,3,10,13\}$ is a base block of a $(20,4,2)$-BIBD. This short orbit base block generates ten blocks and $\operatorname{gcd}(3,10)=1$. The cycle $U=0,3,6,9,2,5,8,1,4,7$ is a length-10 partial Ucycle representing the blocks of $\operatorname{Dev}(\{0,3,10,13\})$ in the order given in Table 15. Note that each of the first three blocks is represented by the first two points in the block, but, the block $\{9,12,19,2\}$ is represented by $\{9,2\}$, where 
the 2 is obtained by adding $3+3+3=9$ to 13 , which is the last point in the first block. This change in the position (within a block) of the representative points also occurs for blocks $\{8,11,18,1\}$ and $\{7,10,17,0\}$.

$\begin{array}{ll}\{0,3,10,13\} & \{5,8,15,18\} \\ \{3,6,13,16\} & \{8,11,18,1\} \\ \{6,9,16,19\} & \{1,4,11,14\} \\ \{9,12,19,2\} & \{4,7,14,17\} \\ \{2,5,12,15\} & \{7,10,17,0\}\end{array}$

Table 15: The blocks of $\operatorname{Dev}(\{0,3,10,13\})$ in the order (read column-wise) given by the length-10 partial Ucycle $U=0,3,6,9,2,5,8,1,4,7$

Lemma 4.2.11. Let $S$ be a cyclic $(v, k, \lambda)$-BIBD, $k \geq 3$, and let $B_{d}=\left\{0, d_{1}, \ldots\right.$ $\left.\ldots, d_{(k-1)}\right\}$ be a non-regular short orbit base block of $S$. Let $d_{1}$ be a point that is not a multiple of $\left|\operatorname{Dev}\left(B_{d}\right)\right|$. If $\operatorname{gcd}\left(d_{1},\left|\operatorname{Dev}\left(B_{d}\right)\right|\right)>1$, then there exists a collection of partial Ucycles (at least two) which considered together include each of the blocks of $\operatorname{Dev}\left(B_{d}\right)$ exactly once. If the partial Ucycles are each of length $\ell$ (they will all be the same length) there will be $\left|\operatorname{Dev}\left(B_{d}\right)\right| / \ell$ partial Ucycles in the collection.

Proof. Let $S=(V, \mathcal{B})$ be a $(v, k, \lambda)$-BIBD, $k \geq 3$, and let $B_{d}=\left\{0, d_{1}, \ldots, d_{(k-1)}\right\}$ be a non-regular short orbit base block of $S$. Let $n=\left|\operatorname{Dev}\left(B_{d}\right)\right|$ and let $m \in \mathbb{Z}_{v}$ be the smallest $m$ such that $m n \equiv 0(\bmod v)$. Suppose $\operatorname{gcd}\left(d_{1}, n\right)>1, n \nmid d_{1}$, and let $c=\operatorname{lcm}\left(n, d_{1}\right)$. There exist $x, \ell \in \mathbb{Z}$ such that $x n=c$ and $\ell d_{1}=c$. The cyclic sequence $U_{0}=0, d_{1}, 2 d_{1}, \ldots,(\ell-1) d$, with multiplication performed modulo $n$, 
contains $\ell$ unique elements of $V$ and thus $\ell$ unique pairs of consecutive elements. As in Lemma 4.2.5, each consecutive pair of points in $U_{0}$ represents a distinct block of $\operatorname{Dev}\left(B_{d}\right) ;$ however, in this case we must show that $\left\{x d_{1}(\bmod n),(x+1) d_{1}(\bmod n)\right\}$ represents $x d_{1}(\bmod n)+B_{d}, x \in\{0,1, \ldots,(n-1)\}$. Let $y$ be the least residue of $x d_{1}$ modulo $n$. If $y+d_{1}(\bmod v)<n$, then the pair $\left\{y, y+d_{1}(\bmod n)\right\}$ are clearly in $y+B_{d}$. On the other hand, if $y+d_{1}(\bmod v)>n$, then we must show that $y+d_{1}(\bmod n)$ appears in $y+B_{d}$. Note that $y+d_{1} \equiv y+d_{1}-n(\bmod n)$ and that $y+d_{1}-n \equiv y+d_{1}+(m-1) n(\bmod v)$. The structure of non-regular short orbit base blocks implies that $y+\left(d_{1}+(m-1) n\right) \in y+B_{d}$ since the point $d_{1}+(m-1) n \in B_{d}$. Therefore, both points $y$ and $y+d_{1}(\bmod n)$ appear in $y+B_{d}$.

For each $i \in\{1,2, \ldots,((n / \ell)-1)\}$, define $U_{i}$ to be the sequence obtained by taking each element of $U_{0}$ and adding $i$ to it. Consider the elements in $U_{0}$ as a subgroup of the group $\left(\mathbb{Z}_{n},+\right)$. The elements in each $U_{i}$ form a coset of $U_{0}$ in $\mathbb{Z}_{n}$, therefore, each element of $\mathbb{Z}_{n}$ appears exactly once in the collection $U\left(B_{d}, d_{1}\right)=$ $\left\{U_{0}, U_{1}, \ldots, U_{(n / \ell)-1}\right\}$. The pair $\left\{x d_{1}+i,(x+1) d_{1}+i\right\}$ (on $\left.U_{i}\right)$ represents the block $\left(x d_{1}+i\right)+B_{d}$, so each pair of consecutive points in these cycles represents a unique block of $\operatorname{Dev}\left(B_{d}\right)$ and every block of $\operatorname{Dev}\left(B_{d}\right)$ is represented exactly once by the collection $U\left(B_{d}, d_{1}\right)$. Finally, the blocks represented by consecutive pairs of points in each $U_{i}, 0 \leq i \leq(n / \ell-1)$, have property $\Pi$. This follows by the same reasoning as in the proof of Lemma 4.2.10. 
Suppose $\{0,10,20,2,12,22\}$ is a base block of a $(30,6,2)$-BIBD. This short orbit base block generates ten blocks and no point in the base block is coprime to ten. Select 2 as the difference with which to create the collection of partial Ucycles. As $\operatorname{lcm}(2,10)=10$, the blocks of $\operatorname{Dev}(\{0,10,20,2,12,22\})$ can be represented by two partial Ucycles, each of length five. The partial Ucycles $U_{0}=0,2,4,6,8$ and $U_{1}=1,3,5,7,9$ represent the ordering of blocks given in Table 16 .

$\begin{array}{cc}U_{0} & U_{1} \\ \{0,10,20,2,12,22\} & \{1,11,21,3,13,23\} \\ \{2,12,22,4,14,24\} & \{3,13,23,5,15,25\} \\ \{4,14,24,6,16,26\} & \{5,15,25,7,17,27\} \\ \{6,16,26,8,18,28\} & \{7,17,27,9,19,29\} \\ \{8,18,28,10,20,0\} & \{9,19,29,11,21,1\}\end{array}$

Table 16: The blocks of $\operatorname{Dev}(\{0,10,20,2,12,22\})$ in the order given by the collection of length-5 partial Ucycles $U_{0}=0,2,4,6,8$ and $U_{1}=1,3,5,7,9$

As the blocks developed from fewer than $k$ copies of a regular short orbit base block cannot be represented in a partial Ucycle created by developing one of the points in the base block, we turn to inserting these blocks into existing collections of partial Ucycles. Some insertion methods require a collection of partial Ucycles representing the blocks developed from a full orbit base block, while others can be executed in collections of partial Ucycles representing blocks developed from a full or a non-regular short orbit base block. If we need not distinguish between full orbit and non-regular short orbit base blocks, we group these two types of base block under 
the heading non-regular base blocks. The lower bounds on the number of full orbit and non-regular short orbit base blocks in the statement of Theorem 4.2 .3 are required to ensure that there will always be enough partial Ucycles to allow for the insertion of all regular short orbit blocks. When inserting into a collection of partial Ucycles representing the blocks of a full orbit base block, $B_{d}=\left\{0, d_{1}, \ldots, d_{(k-1)}\right\}$, it is useful to keep the following rule in mind. Let $g=\operatorname{gcd}\left(d_{1}, v\right)$ and suppose $d_{1}$ was used to form a collection of length- $\ell$ partial Ucycles, $U\left(B_{d}, d_{1}\right)$, representing the blocks developed from $B_{d}$. If $g=1$, then $\ell=v$ and $U\left(B_{d}, d_{1}\right)$ is a single full length partial Ucycle. If $g>1$, then $U\left(B_{d}, d_{1}\right)$ is a collection of length- $\ell$ partial Ucycles, where $\ell \leq v / 2$. The number of partial Ucycles in $U\left(B_{d}, d_{1}\right)$ is $g$.

Blocks developed from a regular short orbit base block will always be inserted into partial Ucycles so that consecutive blocks in the new sequence intersect. Thus, when adding points to a partial Ucycle or when joining partial Ucycles together, it remains to avoid creating the claw configuration on three consecutive blocks. Our formulation of a Ucycle as a list of points from $V$ allows us to avoid claw creation altogether (i.e. we will always maintain property $\Pi$ ). A claw involving the point $x$ would be represented by the sequence $y, x, x, z$, where $x \neq y, z$. However, as each consecutive pair of points in the sequence represents a block containing this pair of points, this sequence implies that some block contains the point $x$ twice - impossible. We now present several lemmas dealing with how and when we can insert regular short 
orbit blocks.

Lemma 4.2.12. Let $S$ be a cyclic $(v, k, \lambda)-B I B D, k \geq 3$. Every full orbit base block in $S$ contains a difference that is not a multiple of $v / k$ modulo $v$.

Proof. Let $S$ be a cyclic $(v, k, \lambda)$-BIBD, $k \geq 3$. The set $W=\{0, v / k, 2 v / k, \ldots,(k-$ 1) $v / k\}$ consists of the unique multiples of $v / k$ modulo $v$. Suppose there were a full orbit block in $S$, denoted $B_{d}$, having all its differences in $W$. Without loss of generality, we may assume that $0 \in B_{d}$, which implies that all points in $B_{d}$ are in $W$. As there are $k$ points in $W$ and $B_{d}$ must have $k$ unique points, $B_{d}=W$ and therefore, $B_{d}$ is a regular short orbit base block. This contradicts our assumption that $B_{d}$ is a full orbit base block.

Lemma 4.2.13. Let $S$ be a cyclic $(v, k, \lambda)-B I B D, k \geq 3$, with a regular short orbit base block $B_{e}$ and a full orbit base block $B_{d}=\left\{0, d_{1}, \ldots, d_{(k-1)}\right\}$. Select a difference $d_{s} \in B_{d}$ that is not a multiple of $v / k$ modulo $v$. Let $U\left(B_{d}, d_{1}\right)=\left\{U_{0}, U_{1}, \ldots, U_{m-1}\right\}$,

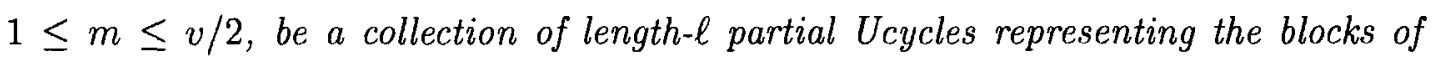
$\operatorname{Dev}\left(B_{d}\right)$, with $d_{1} \neq d_{s}$ used to form the partial Ucycles. Two distinct blocks of $\operatorname{Dev}\left(B_{e}\right)$ can be inserted into the partial Ucycles of $U\left(B_{d}, d_{1}\right)$.

Proof. Let $S$ be a cyclic $(v, k, \lambda)$-BIBD, $k \geq 3$, and let $B_{d}=\left\{0, d_{1}, \ldots, d_{(k-1)}\right\}$ be a full orbit base block of the design. Select a difference $d_{s}$ from $B_{d}$ that is not a multiple of $v / k$ modulo $v$. Lemma 4.2 .12 implies that such a $d_{s}$ must exist. Let 


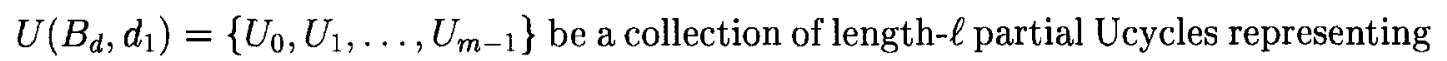
the blocks of $\operatorname{Dev}\left(B_{d}\right)$ with $d_{1} \neq d_{s}$ used to form the partial Ucycles. We will insert two blocks of $\operatorname{Dev}\left(B_{e}\right)$ into the partial Ucycles of $U\left(B_{d}, d_{1}\right)$. The blocks we insert are of the form $x+\{0, v / k, \ldots,(k-1) v / k\}$ and $\left(x+d_{s}\right)+\{0, v / k, \ldots,(k-1) v / k\}$, for some $x \in\{0,1, \ldots, v / k-1\}$. These two blocks are distinct because the only way to obtain a copy of $x+B_{e}$ is to add a multiple of $v / k$ to $x+B_{e}$.

Select two points $y, z \in x+\{0, v / k, \ldots,(k-1) v / k\}, y \neq z$, to represent the block $x+B_{e}$. Then $\left(x+d_{s}\right)+\{0, v / k, \ldots,(k-1) v / k\}$ will be represented by the pair $\left\{y+d_{s}, z+d_{s}\right\}$. As each point of $\mathbb{Z}_{v}$ appears in a partial Ucycle of $U\left(B_{d}, d_{1}\right)$ exactly once (refer to Lemmas 4.2.4 and 4.2.5), there exists $i \in\{0, \ldots, m-1\}$ such that $y \in U_{i}$ and there exists $j \in\{0, \ldots, m-1\}$ such that $z \in U_{j}$. The method used to insert these two blocks depends on the relationship of $i$ to $j$.

Case 1: If $i=j$, then $y$ and $z$ appear in the same partial Ucycle of $U\left(B_{d}, d_{1}\right)$. Represent $y$ and $z$ in terms of $d_{1}: y=a d_{1}+i$ and $z=c d_{1}+i$, for $a, c \in \mathbb{Z}_{v}$. Without loss of generality, assume $a<c$. To insert the blocks represented by $\{y, z\}$ and $\left\{y+d_{s}, z+d_{s}\right\}$ into $U_{i}$, break $U_{i}$ between the points $a d_{1}+i$ and $(a+1) d_{1}+i$ and between the points $(c-1) d_{1}+i$ and $c d_{1}+i$. We now have two segments: $(a+1) d_{1}+i,(a+2) d_{1}+i, \ldots,(c-1) d_{1}+i$ and $c d_{1}+i,(c+1) d_{1}+i, \ldots, a d_{1}+i$ Add $a d_{1}+i$ to the beginning of the first segment and $c d_{1}+i$ to the end of the first segment, yielding the segment $a d_{1}+i,(a+1) d_{1}+i,(a+2) d_{1}+i, \ldots,(c-1) d_{1}+i, c d_{1}+i$. 
Reverse the orientation of this segment and reattach it to the segment running from $c d_{1}+i$ to $a d_{1}+i$. Now the partial Ucycle has two pairs of consecutive points equal to $\left\{a d_{1}+i, c d_{1}+i\right\}$, therefore, change the $a d_{1}+i$ adjacent to $(a+1) d_{1}+i$ to $a d_{1}+d_{s}+i$ and change the $c d_{1}+i$ adjacent to $(c+1) d_{1}+i$ to $c d_{1}+d_{s}+i$. We have added two new blocks to the partial Ucycle; the first is represented by the pair $\left\{a d_{1}+i, c d_{1}+i\right\}=$ $\{y, z\}$ and the second by $\left\{a d_{1}+d_{s}+i, c d_{1}+d_{s}+i\right\}=\left\{y+d_{s}, z+d_{s}\right\}$. All of the blocks represented by the original partial Ucycle $U_{i}$ remain intact; however, the block $i+\left\{a d_{1},(a+1) d_{1}, a d_{1}+d_{2}, \ldots, a d_{1}+d_{s}, \ldots, a d_{1}+d_{(k-1)}\right\}$ which was originally represented by the pair $\left\{a d_{1}+i,(a+1) d_{1}+i\right\}$ is now represented by $\left\{a d_{1}+d_{s}+i,(a+\right.$ 1) $\left.d_{1}+i\right\}$ and the block $i+\left\{c d_{1},(c+1) d_{1}, c d_{1}+d_{2}, \ldots, c d_{1}+d_{s}, \ldots, c d_{1}+d_{(k-1)}\right\}$ which was originally represented by the pair $\left\{c d_{1}+i,(c+1) d_{1}+i\right\}$ is now represented by $\left\{c d_{1}+d_{s}+i,(c+1) d_{1}+i\right\}$. We will refer to this type of insertion as Insertion $\boldsymbol{A}$.

The procedure is better illustrated with a picture. In Figure 20, green lines and numbers represent changes to the original partial Ucycle - the green lines are new connections and the green numbers are altered numbers (with original numbers in square brackets). The gray letters and numbers represent the segment of the original partial Ucycle that has been reversed (and which appears in its new orientation at the top of the figure).

Note that our choice of the segment $a d_{1}+i,(a+1) d_{1}+i, \ldots,(c-1) d_{1}+i, c d_{1}+i$ for reversal is arbitrary. We could just as easily reverse the segment $c d_{1}+i,(c+1) d_{1}+$ 


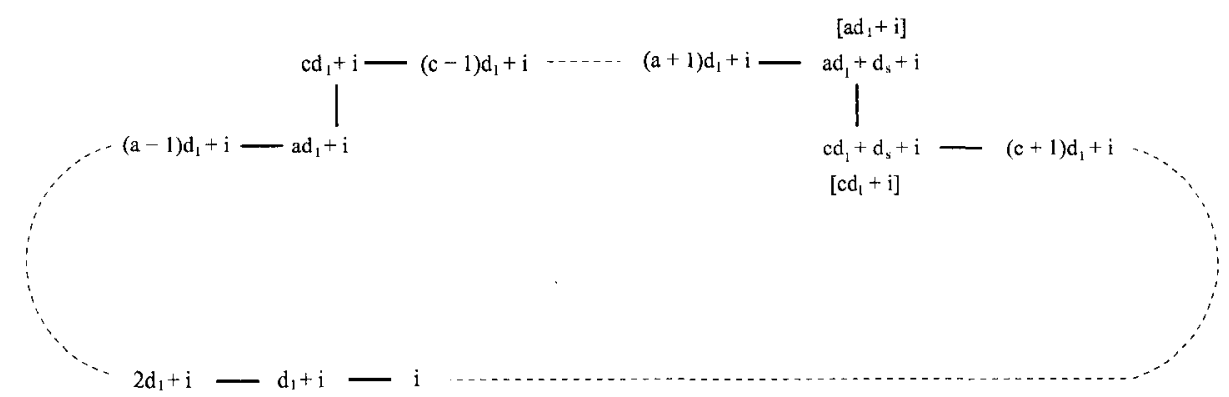

Figure 20: Insertion $A$ : two distinct blocks developed from a regular short orbit base block are inserted into a single partial Ucycle

$i, \ldots,(a-1) d_{1}+i, a d_{1}+i$. As above, we would then change the $a d_{1}+i$ adjacent to $(a+1) d_{1}+i$ to $a d_{1}+d_{s}+i$ and change the $c d_{1}+i$ adjacent to $(c+1) d_{1}+i$ to $c d_{1}+d_{s}+i$

Case 2: If $i \neq j$, then $y$ and $z$ appear in different partial Ucycles of $U\left(B_{d}, d_{1}\right)$. Represent $y$ and $z$ in terms of $d_{1}: y=a d_{1}+i$ and $z=c d_{1}+j$, for $a, c \in \mathbb{Z}_{v}$. To insert the blocks represented by $\{y, z\}$ and $\left\{y+d_{s}, z+d_{s}\right\}$, break $U_{i}$ between the points $a d_{1}+i$ and $(a+1) d_{1}+i$ and break $U_{j}$ between the points $c d_{1}+j$ and $(c+1) d_{1}+j$.

We now have two segments: $(a+1) d_{1}+i,(a+2) d_{1}+i, \ldots, a d_{1}+i$ and $(c+$ 1) $d_{1}+j,(c+2) d_{1}+j, \ldots, c d_{1}+j$. Add $a d_{1}+i$ to the beginning of the first segment, creating a segment that starts and ends with $a d_{1}+i$. Add $c d_{1}+j$ to the beginning of the second segment, creating a segment that starts and ends with $c d_{1}+j$. Now reverse the orientation of one of these two segments. Without loss of generality, choose the segment beginning and ending with $c d_{1}+j$. Reattach the segments so that $\ldots,(a-1) d_{1}+i, a d_{1}+i$ is joined to $c d_{1}+j,(c-1) d_{1}+j, \ldots$ and $\ldots,(c+1) d_{1}+j, c d_{1}+j$ 
is joined to $a d_{1}+i,(a+1) d_{1}+i, \ldots$. The two segments now have opposite orientations, that is, if we pass along the sequence $U_{i}$ in the direction such that vertex labels increase by $+d_{1}$, then, as we pass along $U_{j}$ in the same direction, the increments are $-d_{1}$. As in Case 1, at this stage the new partial Ucycle has two pairs of consecutive points equal to $\left\{a d_{1}+i, c d_{1}+j\right\}$, therefore, change the $a d_{1}+i$ adjacent to $(a+1) d_{1}+i$ to $a d_{1}+d_{s}+i$ and change the $c d_{1}+j$ adjacent to $(c+1) d_{1}+j$ to $c d_{1}+d_{s}+j$. We have added two new blocks to the collection of partial Ucycles; the first is represented by the pair $\left\{a d_{1}+i, c d_{1}+j\right\}=\{y, z\}$ and the second by $\left\{a d_{1}+d_{s}+i, c d_{1}+d_{s}+j\right\}=\left\{y+d_{s}, z+d_{s}\right\}$. All of the blocks represented by the two original partial Ucycles $U_{i}$ and $U_{j}$ remain intact, however, the block $i+\left\{a d_{1},(a+1) d_{1}, a d_{1}+d_{2}, \ldots, a d_{1}+d_{s}, \ldots, a d_{1}+d_{(k-1)}\right\}$ which was originally represented by the pair $\left\{a d_{1}+i,(a+1) d_{1}+i\right\}$ is now represented by $\left\{a d_{1}+d_{s}+i,(a+1) d_{1}+i\right\}$ and the block $j+\left\{c d_{1},(c+1) d_{1}, c d_{1}+d_{2}, \ldots, c d_{1}+\right.$ $\left.d_{s}, \ldots, c d_{1}+d_{(k-1)}\right\}$ which was originally represented by the pair $\left\{c d_{1}+j,(c+1) d_{1}+j\right\}$ is now represented by $\left\{c d_{1}+d_{s}+j,(c+1) d_{1}+j\right\}$. We will refer to this type of insertion as Insertion $B$.

The procedure is better illustrated with a picture. In Figure 21, green lines and numbers represent changes to the original Ucycles - the green lines are new connections and the green numbers are altered numbers (with original numbers in square brackets). The blue numbers and lines represent $U_{i}$ and the red letters and numbers represent $U_{j}$. 
CHAPTER 4. GRAY CODES AND UNIVERSAL CYCLES FOR DESIGNS 163

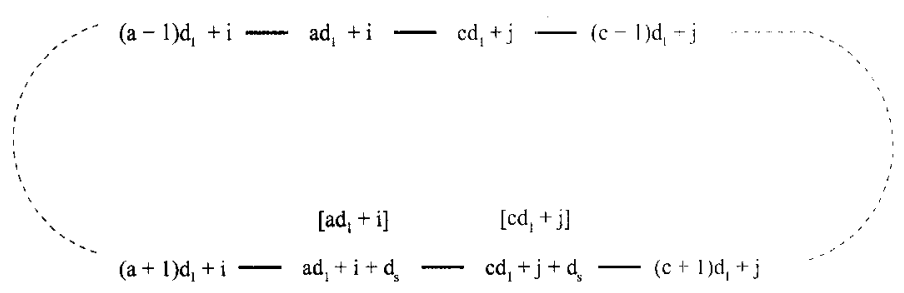

Figure 21: Insertion $B$ : two distinct blocks developed from a regular short orbit base block are inserted between two partial Ucycles from the same collection

We note that the method for inserting blocks into partial Ucycles described in the proof of Lemma 4.2.13 is reminiscent of that employed by Vickers and Silverman in creating balanced Gray codes [55].

It is possible to exactly determine which partial Ucycles the points $y$ and $z$ (defined in the proof of Lemma 4.2.13) appear on. Without loss of generality assume the pair of points representing one of the insertion blocks is $y$ and $z=y+v / k$. Suppose we have the collection of partial Ucycles $U\left(B_{d}, d_{1}\right)=\left\{U_{0}, U_{1}, \ldots, U_{m-1}\right\}$ representing the blocks of $\operatorname{Dev}\left(B_{d}\right)$, where $B_{d}=\left\{0, d_{1}, d_{2}, \ldots, d_{(k-1)}\right\}$. Further, suppose $d_{s} \in B_{d}$ with $d_{s} \neq d_{1}$ and $d_{s} \neq r \cdot v / k$, for all $r \in\{0,1, \ldots,(k-1)\}$. As each point of $\mathbb{Z}_{v}$ appears in a partial Ucycle of $U\left(B_{d}, d_{1}\right)$ exactly once, there exists $i \in\{0, \ldots, m-1\}$ such that $y \in U_{i}$. This implies that $y=a d_{1}+i$, for some $a \in \mathbb{Z}_{v}$. On which sequence of $U\left(B_{d}, d_{1}\right)$ does $y+v / k$ appear? The answer depends on the divisibility of $v$ by $k g$, where $g=\operatorname{gcd}\left(d_{1}, v\right)$ (recall that $d_{1}$ is the difference used to create the collection of partial Ucycles $\left.U\left(B_{d}, d_{1}\right)\right)$. If $k g \mid v$, then $y+v / k$ appears in $U_{i}$. The expression $k g \mid v$ implies $g \mid(v / k)$, thus $q g=v / k$, for some $q \in \mathbb{Z}$. In group theoretic terms, $v / k$ belongs 
to the cyclic subgroup of $\mathbb{Z}_{v}$ generated by $g$, that is $v / k \in\langle g\rangle$. Now $d_{1}=s g$, for some $s \in \mathbb{Z}$, so $d_{1} \in\langle g\rangle$. Any element coprime to the order of the cyclic subgroup can be used to generate the subgroup, therefore, $\langle g\rangle=\left\langle d_{1}\right\rangle$ and $v / k \in\left\langle d_{1}\right\rangle$, which implies that $v / k=t d_{1}, t \in \mathbb{Z}$. Since $y=a d_{1}+i$, we have $y+v / k=a d_{1}+i+v / k=(a+t) d_{1}+i$, therefore, $y+v / k$ is a point in $U_{i}$. Following the same argument, we can show that if $k g \nmid v$, then $y+v / k$ appears in $U_{i+r}$, where $v / k=q d_{1}+r, r \neq 0$.

Lemma 4.2.14. Let $S=(V, \mathcal{B})$ be a cyclic $(v, k, \lambda)-B I B D, k \geq 3$ and let $B_{d}=$ $\left\{0, d_{1}, \ldots, d_{(k-1)}\right\}$ be a non-regular base block of the design. Let $U\left(B_{d}, d_{1}\right)=\left\{U_{0}, U_{1}\right.$, $\left.\ldots, U_{m-1}\right\}, 1 \leq m \leq v / 2$, be a collection of length-l partial Ucycles representing the blocks of Dev $\left(B_{d}\right)$, with $d_{1}$ used to form the partial Ucycles. If $B_{\boldsymbol{e}}$ is a repeated regular short orbit base block, then two copies of a block in Dev $\left(B_{e}\right)$ can be inserted into the partial Ucycles of $U\left(B_{d}, d_{1}\right)$.

Proof. Let $S$ be a cyclic $(v, k, \lambda)$-BIBD and let $B_{d}=\left\{0, d_{1}, \ldots, d_{(k-1)}\right\}$ be a full orbit or non-regular short orbit base block of the design. Let $U\left(B_{d}, d_{1}\right)=\left\{U_{0}, U_{1}, \ldots, U_{m-1}\right\}$ be a collection of length- $\ell$ partial Ucycles representing the blocks of $\operatorname{Dev}\left(B_{d}\right)$, with $d_{1}$ used to form the partial Ucycles. We will insert two blocks of the form $x+B_{e}$, $x \in\{0,1, \ldots, v / k-1\}$. Select points $x$ and $x+v / k$ to represent both copies of $x+B_{e}$. Note that to represent any block in $\operatorname{Dev}\left(B_{e}\right)$, we require only points in $\{0,1, \ldots, 2 v / k-1\}$. As non-regular short orbit base blocks generate at least $2 v / k$ blocks, each of the points in $\{0,1, \ldots, 2 v / k-1\}$ appears in the collection of partial 
Ucycles $U\left(B_{d}, d_{1}\right)$. There exists $i \in\{0, \ldots, m-1\}$ such that $x \in U_{i}$ and there exists $j \in\{0, \ldots, m-1\}$ such that $x+v / k \in U_{j}$. The method used to insert the two blocks depends on the relationship of $i$ to $j$.

Case 1: If $i=j$, then $x$ and $x+v / k$ appear in the same partial Ucycle of $U\left(B_{d}, d_{1}\right)$. Use Insertion $A$ (see Figure 20), but do not change the labels of any points (that is, do not change $a d_{1}$ to $a d_{1}+d_{s}$ nor $c d_{1}$ to $c d_{1}+d_{s}$ ). We will refer to this type of insertion as Insertion $\boldsymbol{A}^{\prime}$.

Case 2: If $i \neq j$, then $x$ and $x+v / k$ appear in different partial Ucycles of $U\left(B_{d}, d_{1}\right)$. Use Insertion $B$ (see Figure 21), but do not change the labels of any points. We will refer to this type of insertion as Insertion $\boldsymbol{B}^{\prime}$.

We have four methods for inserting two regular short orbit blocks into a collection of partial Ucycles at the same time. When we use each of these methods depends on how many copies of the regular short orbit base block appear in the design in question and the difference used to create the collection of partial Ucycles we have chosen to insert into. We would like to perform the types of insertion described in Lemmas 4.2.13 and 4.2.14 multiple times within a given collection of partial Ucycles. As Insertions $A^{\prime}$ and $B^{\prime}$ are the most simple insertions, we prefer to insert as many regular short orbit blocks as possible using these two methods. Suppose $B_{e}$ is a regular short orbit base block in a $(v, k, \lambda)$-BIBD. If $\lambda>1$ and $B_{e}$ is repeated, we pair copies of $B_{e}$ and insert pairs of blocks using Insertions $A^{\prime}$ and $B^{\prime}$. If $\lambda=1$ or we 
have one copy of $B_{e}$ remaining, we pair distinct blocks of $\operatorname{Dev}\left(B_{e}\right)$ and insert pairs by Insertions $A$ and $B$. Notice that if $v / k \equiv 1(\bmod 2)$ one block of $\operatorname{Dev}\left(B_{e}\right)$ will not be paired. Lemma 4.2 .22 deals with how to insert this single block.

In Figures 20 and 21, we illustrated partial Ucycles by writing the points of the Ucycle and connecting each pair of points by an edge. We can think of each edge as representing the block represented by the two endpoints. We will assign a colour to each edge of a partial Ucycle to illustrate the relationship of the two endpoints. Note that these colours do not represent the base block from which the block represented was developed (as in the base block coloured pair adjacency graph); instead, colours will represent the difference between the two endpoints of an edge relative to a fixed direction of reading. Ucycles will always be read clockwise. If, when reading a partial Ucycle in the clockwise direction, the endpoints of the edge increase by $d_{1}$ (the difference used to create the Ucycle), colour the edge blue. If the endpoints of the edge decrease by $d_{1}$, colour the edge red. When a partial Ucycle is first created all of its edges are blue. However, when we insert a pair of blocks into a collection of partial Ucycles, a segment of a partial Ucycle (or an entire partial Ucycle) is reversed. Suppose we wish to insert a pair of blocks where at least one of the blocks is represented by the pair $\{y, z\}, y, z \in \mathbb{Z}_{v}$. To insert this pair of blocks, a single partial Ucycle (or two partial Ucycles in a collection of partial Ucycles) is broken at the point $y$ and at the point $z$ and two segments are created. One of these segments 
is reversed and then the two segments are reattached so that two copies of the edge $\{y, z\}$ have been introduced into the partial Ucycle. If the two blocks inserted are distinct, then one of the $\{y, z\}$ edges has endpoint labels changed to $\left\{y+d_{s}, z+d_{s}\right\}$. We colour the two new edges green and we swap red for blue and blue for red on each edge of the reversed segment. All other edge colours are unchanged.

Lemma 4.2.15. Let $S$ be a cyclic $(v, k, \lambda)-B I B D, k \geq 3$, with a regular short orbit base block $B_{e}$ and a non-regular base block $B_{d}=\left\{0, d_{1}, \ldots, d_{(k-1)}\right\}$. Let $U\left(B_{d}, d_{1}\right)=$ $\left\{U_{0}, U_{1}, \ldots, U_{m-1}\right\}, 1 \leq m \leq v / 2$, be a collection of length- $\ell$ partial Ucycles representing the blocks of Dev $\left(B_{d}\right)$, with $d_{1}$ used to form the partial Ucycles. If $B_{e}$ appears twice in $S$, then two complete sets of blocks developed from $B_{e}$ can be inserted into the partial Ucycles of $U\left(B_{d}, d_{1}\right)$.

Proof. Let $S$ be a cyclic $(v, k, \lambda)$-BIBD, $k \geq 3$, with full orbit or non-regular short orbit base block $B_{d}=\left\{0, d_{1}, \ldots, d_{(k-1)}\right\}$. Let $U\left(B_{d}, d_{1}\right)=\left\{U_{0}, U_{1}, \ldots, U_{m-1}\right\}$ be a collection of length- $\ell$ partial Ucycles representing the blocks of $\operatorname{Dev}\left(B_{d}\right)$, with $d_{1}$ used to form the partial Ucycles. Suppose that $B_{e}$ is a repeated regular short orbit base block in $S$. For each $x \in\{0,1, \ldots, v / k-1\} \subset \mathbb{Z}_{v}$, we will insert the block $x+B_{e}$ twice into the partial Ucycles of $U\left(B_{d}, d_{1}\right)$ using insertion method $A^{\prime}$ or $B^{\prime}$. We must ensure that we never wish to insert a regular short orbit block at a point that has been involved in a previous insertion. Choose $\{0, v / k\}$ to represent $B_{e}$. For each $x \in\{0,1, \ldots, v / k-1\}$, the block $x+B_{e}$ will be represented by $\{x, v / k+x\}$. 
These pairs are pairwise disjoint, therefore, each block of $\operatorname{Dev}\left(B_{e}\right)$ is inserted at a unique pair of points and so two copies of each block in $\operatorname{Dev}\left(B_{e}\right)$ can be inserted into $U\left(B_{d}, d_{1}\right)$. In visual terms, the disjointness of the insertion pairs implies that we never wish to insert at a point incident to a green edge.

Corollary 4.2.16. Let $S$ be a cyclic $(v, k, \lambda)-B I B D, k \geq 3$, with regular short orbit base block $B_{e}$. If $n_{3}=2$ and $n_{1}+n_{2} \geq 1$, then there exists a collection of length- $\ell$ partial Ucycles representing the blocks developed from a non-regular base block into which all blocks from two copies of $\operatorname{Dev}\left(B_{e}\right)$ can be inserted.

If there are an odd number of regular short orbit base blocks in a BIBD, we must insert one of the sets of $\operatorname{Dev}(\{0, v / k, \ldots,(k-1) v / k\})$ singly. This will require the use of insertion methods $A$ or $B$ and the existence of a full orbit base block containing a difference coprime to $v / k$.

Lemma 4.2.17. Let $S$ be a cyclic $(v, k, \lambda)-B I B D, k \geq 3$. There exist at least

$$
\left\lceil\frac{\lambda \phi(v / k)}{k-1}\right\rceil-n_{2}
$$

full orbit base blocks each containing a difference coprime to $v / k$.

Proof. Let $S$ be a cyclic $(v, k, \lambda)$-BIBD, $k \geq 3$. The number of elements in $\mathbb{Z}_{v}$ that are coprime to $v / k$ is $k \cdot \phi(v / k)$. The total number of such elements appearing as differences in the base blocks of $S$ is $\lambda k \phi(v / k)$. Notice that none of these differences can be in regular short orbit base blocks, therefore, the minimum number of non-regular 
base blocks in $S$ containing differences coprime to $v / k$ (differences not necessarily all unique) is

$$
\left\lceil\frac{\lambda k \phi(v / k)}{2\left(\begin{array}{l}
k \\
2
\end{array}\right)}\right\rceil,
$$

since the number of differences appearing in a base block is $2\left(\begin{array}{l}k \\ 2\end{array}\right)$. To ensure that $x$ of the base blocks containing a difference coprime to $v / k$ are full orbit base blocks, we must have $x \leq\lceil(\lambda \phi(v / k)) /(k-1)\rceil-n_{2}$.

The proof of the Theorem 4.2.3 will require the existence of full orbit base blocks containing differences coprime to $v$ (not $v / k$, as stated above in Lemma 4.2.17 and in several of the following lemmas). Of course, any difference coprime to $v$ is coprime to $v / k$, therefore, in the proof of Theorem 4.2 .3 we will appeal to the following corollary.

Corollary 4.2.18. Let $S$ be a cyclic $(v, k, \lambda)-B I B D, k \geq 3$. There exist at least

$$
\left\lceil\frac{\lambda \phi(v)}{k(k-1)}\right\rceil-n_{2}
$$

full orbit base blocks each containing a difference coprime to $v$.

Lemma 4.2.19. Let $S$ be a cyclic $(v, k, \lambda)$-BIBD, $k \geq 3$, with a regular short orbit base block $B_{e}$. If

$$
x \leq\left\lceil\frac{\lambda \phi(v / k)}{(k-1)}\right\rceil-n_{2}
$$

and $x \geq 1$, then there exists a collection of length- $\ell$ partial Ucycles representing the blocks generated by a full orbit base block into which all blocks of Dev( $\left.B_{e}\right)$, except 
possibly one, can be inserted without using the partial Ucycles representing a predetermined set of $x-1$ full orbit base blocks.

Proof. Let $S$ be a cyclic $(v, k, \lambda)$-BIBD, $k \geq 3$, with $n_{2} \leq\lceil(\lambda \phi(v / k)) /(k-1)\rceil-x$, for some $x \geq 1$. Lemma 4.2 .17 implies that there exists a full orbit base block in $S$, denoted $B_{d}=\left\{0, d_{1}, \ldots, d_{(k-1)}\right\}$, which is distinct from $x-1$ predetermined full orbit base blocks and which contains at least one difference coprime to $v / k$. Let $d_{s} \in B_{d}$ be a difference coprime to $v / k$ and let $U\left(B_{d}, d_{1}\right)=\left\{U_{0}, U_{1}, \ldots, U_{m-1}\right\}$ be a collection of length- $\ell$ partial Ucycles representing the blocks of $\operatorname{Dev}\left(B_{d}\right)$, with $d_{1} \neq d_{s}$ used to form the partial Ucycles.

Suppose $B_{e}$ is a regular short orbit base block in $S$. The points in the blocks of $\operatorname{Dev}\left(B_{e}\right)$ are disjoint, therefore we will use Insertions $A$ and $B$ to insert blocks of $\operatorname{Dev}\left(B_{e}\right)$ into partial Ucycles. We pair the blocks of $\operatorname{Dev}\left(B_{e}\right)$ as follows: pair $B_{e}$ with $d_{s}+B_{e}$, next pair $2 d_{s}+B_{e}$ with $3 d_{s}+B_{e}$, and so on, where the multiplication $x \cdot d_{s}$ is taken modulo $v / k$. As $\operatorname{gcd}\left(v / k, d_{s}\right)=1$, at most one of the blocks in $\operatorname{Dev}\left(B_{e}\right)$ is not paired. We prove that all the pairs can be inserted into $U\left(B_{d}, d_{1}\right)$.

Prior to insertion, we do not know which pair of points we will use to represent any of the blocks in $\operatorname{Dev}\left(B_{e}\right)$; however, because the blocks of $\operatorname{Dev}\left(B_{e}\right)$ are disjoint there is no possibility of trying to make an insertion involving a point that has already been used for insertion. Which points are selected to represent the block $2 x d_{s}+B_{e}, x \in \mathbb{Z}$, will be determined by the colours of the edges in $U\left(B_{d}, d_{1}\right)$ incident to each of the 
points in $2 x d_{s}+B_{e}$ at the time of insertion. Since the blocks of $\operatorname{Dev}\left(B_{e}\right)$ are disjoint, none of the points in $2 x d_{s}+B_{e}$ will be incident to a green edge before $2 x d_{s}+B_{e}$ is inserted. Furthermore, since every insertion creates a green edge between the two segments of partial Ucycles involved in the insertion, every sequence of blue edges is separated from every sequence of red edges by a green edge. That is, blue and red edges are never incident to each other. As a result, each pair of edges incident to each of the points in $2 x d_{s}+B_{e}$ must either both be blue or both be red. There are at least three unique points in $2 x d_{s}+B_{e}$, so in $U\left(B_{d}, d_{1}\right)$ at least two of these points have incident edges of the same colour.

Select two points in $2 x d_{s}+B_{e}$ that have edges of the same colour incident to them in $U\left(B_{d}, d_{1}\right)$. Denote the pair $\left\{a d_{1}+i, c d_{1}+j\right\}, a, c \in \mathbb{Z}_{v}$, where $i=j$ if the pair of points is in the same partial Ucycle of $U\left(B_{d}, d_{1}\right)$ and $i \neq j$ if each point is in a different partial Ucycle of $U\left(B_{d}, d_{1}\right)$. Without loss of generality, assume $a \leq c$ ( $a$ may be equal to $c$ only if $i \neq j$ ). This pair of points will be used to represent $2 x d_{s}+B_{e}$. As we are employing insertions $A$ and $B$, when we insert $2 x d_{s}+B_{e}$ we will also insert $d_{s}+2 x d_{s}+B_{e}$ and this block will be represented by the pair $\left\{a d_{1}+i+d_{s}, c d_{1}+j+d_{s}\right\}$. Recall that the rule for determining which copy of the introduced edge $\left\{a d_{1}+i, c d_{1}+j\right\}$ will be changed to $\left\{a d_{1}+i+d_{s}, c d_{1}+j+d_{s}\right\}$ is change the $a d_{1}+i$ adjacent to $(a+1) d_{1}+i$ to $a d_{1}+d_{s}+i$ and change the $c d_{1}+j$ adjacent to $(c+1) d_{1}+j$ to $c d_{1}+d_{s}+j$. This change ensures that the block $a d_{1}+i+B_{d}$ 
which had previously been represented by the edge $\left\{a d_{1}+i,(a+1) d_{1}+i\right\}$, is still in the partial Ucycle but is now represented by the pair $\left\{a d_{1}+i+d_{s},(a+1) d_{1}+i\right\}$. Similarly, $c d_{1}+j+B_{d}$ is now represented by the pair $\left\{c d_{1}+j+d_{s},(c+1) d_{1}+j\right\}$. In colouring terms, this means that we change the $\left\{a d_{1}+i, c d_{1}+i\right\}$ edge which (when reading the Ucycle in the clockwise direction) has a red edge preceding it and a blue edge following it.

To ensure that multiple insertions can be made into $U\left(B_{d}, d_{1}\right)$ we must ensure that when we change point labels the edges incident to these changed points continue to represent the same blocks. Our argument is based on the rule used to determine which of the two new green edges has its endpoints changed when performing an insertion of two distinct blocks. The rule says we change the endpoints of a green edge if it has a red edge preceding it and a blue edge following it. Note that this edge colour order (red, green, blue) is independent of reversal of any segment containing these three edges since reversal of a segment swaps the colour of red and blue edges. Furthermore, any segment to be reversed that contains one of these three edges must contain all of these edges.

Without loss of generality, suppose $a d_{1}+i+d_{s}$ is the changed point adjacent to the red edge. Then the edge colour-point sequence around the changed edge is red edge, $a d_{1}+i+d_{s}$, green edge, $c d_{1}+j+d_{s}$, blue edge. For the red edge to represent $a d_{1}+i+B_{d}$ its other endpoint must be $(a+1) d_{1}+i$. That is, the other endpoint must 
not be a changed point. Denote this endpoint by $w$. If $w$ were a changed point then the other edge it is adjacent to would be green. However, the change rule says that we change the endpoints of the green edge if it has a blue edge following it, therefore, $w$ must not be a changed point. Similarly, since $c d_{1}+j+d_{s}$ is the changed point adjacent to the blue edge, the other endpoint of the blue edge must be $(c+1) d_{1}+j$, in order for the blue edge to continue to represent $c d_{1}+j+B_{d}$. Denote this other endpoint of the blue edge by $w^{\prime}$. If $w^{\prime}$ were a changed point then the other edge it is adjacent to would be green. However, the change rule says that we change the endpoints of the green edge if it has a red edge preceding it, therefore, $w^{\prime}$ must not be a changed point. We conclude that all paired blocks of $B_{e}$ can be inserted into $U\left(B_{d}, d_{1}\right)$ without affecting the blocks represented by the original partial Ucycles in $U\left(B_{d}, d_{1}\right)$. Figure 22 illustrates this argument. The circles represent unknown points that have no impact on the argument.

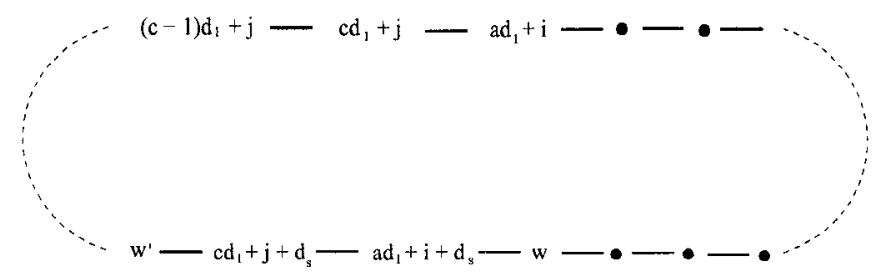

Figure 22: Insertion of multiple pairs of blocks developed from a regular short orbit base block into a collection of partial Ucycles

We restate Lemma 4.2.19 in terms of $\phi(v)$, instead of $\phi(v / k)$.

Corollary 4.2.20. Let $S$ be a cyclic $(v, k, \lambda)-B I B D, k \geq 3$, with regular short orbit 
base block $B_{e}$. If $x \leq\lceil\lambda \phi(v) /(k(k-1))\rceil-n_{2}$, and $x \geq 1$, then there exists a collection

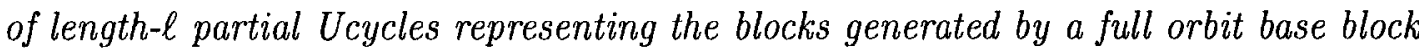
into which all blocks of Dev $\left(B_{e}\right)$, except possibly one, can be inserted without using the partial Ucycles representing a predetermined set of $x-1$ full orbit base blocks.

With Lemmas 4.2 .15 and 4.2.19, we have established that all, or all save one, blocks developed from a regular short orbit base block can be inserted into an existing collection of partial Ucycles. The following two lemmas deal with insertion of a single regular short orbit block. Note that the type of insertion we describe will need to be executed only if both $n_{3}$ and $v / k$ are odd. Lemmas 4.2 .21 and 4.2 .22 rely on these parity assumptions. Before introducing the final type of insertion, Lemma 4.2.21 establishes when the properties necessary for execution of this insertion hold. To aid our discussion, we will say a difference $d$ is unusable if all $\lambda$ copies of each difference $d \pm r \cdot v / k(\bmod v), r \in \mathbb{Z}_{k}$, appear in the same non-regular base block.

Lemma 4.2.21. Let $S$ be a cyclic $(v, k, \lambda)-B I B D, k \geq 3$. If the number of regular short orbit base blocks in $S$ is odd, $v / k$ is odd, and the number of non-regular base blocks in $S$ is at least two, there exist non-regular base blocks $B_{d}=\left\{0, d_{1}, \ldots, d_{(k-1)}\right\}$ and $B_{f}=\left\{0, f_{1}, \ldots, f_{(k-1)}\right\}$ such that $f_{t}-d_{s}$ is equal to a difference in a regular short orbit base block for some $s, t \in\{1,2, \ldots,(k-1)\}$.

Proof. Let $S$ be a cyclic $(v, k, \lambda)$-BIBD, $k \geq 3$, and let $B_{e}$ be a regular short orbit base block of the design. We wish to find differences $d_{s}, f_{t} \in \mathbb{Z}_{v} \backslash\{0\}$, with these 
CHAPTER 4. GRAY CODES AND UNIVERSAL CYCLES FOR DESIGNS 175

differences appearing in two different non-regular base blocks, such that $f_{t}-d_{s}$ is a difference in $B_{e}$. Consider $W=\{0, v / k, 2 v / k, \ldots,(k-1) v / k\}-$ the differences in a regular short orbit base block union $\{0\}-$ as a subgroup of $\mathbb{Z}_{v}$. The cosets $W$, $W \pm 1 \ldots, W \pm(v-k) / 2 k$ are disjoint and partition $\mathbb{Z}_{v}$. If a difference $d_{s} \in \mathbb{Z}_{v} \backslash\{0\}$ is unusable, all $\lambda$ copies of each of the differences in $W-d_{s}$ and $W+d_{s}$ must be in the same non-regular base block.

Suppose every difference in $\mathbb{Z}_{v}$ is unusable. For each difference in a base block, $\lambda$ copies of the coset containing this difference and $\lambda$ copies of the coset containing its negative must appear in the base block. The differences in a pair of cosets of the form $W+d, W-d$, must completely fit in a single base block and no portion of a coset may appear in another base block. If the number of differences in a non-regular base block is not divisible by $2 k \lambda$, or if the cosets in the base block do not account for all differences in the base block, then the remaining differences in the base block are multiples of $v / k$. We break our analysis into two cases.

Case 1: Suppose $\lambda=1$. $S$ must contain exactly one regular short orbit base block and the remaining blocks of the design must be full orbit base blocks. The differences in $W \backslash\{0\}$ appear only in the regular short orbit base block. Furthermore, the cosets $W \pm i, i \in\{1,2, \ldots,(v-k) / 2 k\}$ pack perfectly into the full orbit base blocks because no multiples of $v / k$ are available to complete the packing. If a full orbit base block contains an element of $W+d, d \in\{1,2, \ldots,(v-k) / 2 k\}$, the block 
must contain all differences in $W+d$ and $W-d$. Therefore, since each coset contains $k$ differences and each full orbit base block contains $2\left(\begin{array}{l}k \\ 2\end{array}\right)$ differences, we must have $2 k \mid 2\left(\begin{array}{l}k \\ 2\end{array}\right)$, which implies $2 \mid(k-1)$. Thus, $k$ must be odd, and since $v / k$ is also odd, $v$ must be odd. Consider a full orbit base block $B$ and, without loss of generality, assume $0 \in B$. Let $B^{d}$ denote the set of differences in $B$. Since the cosets must pack exactly, $B^{d}=\left\{W-x_{1}, W+x_{1}, W-x_{2}, W+x_{2}, \ldots, W-x_{(k-1) / 2}, W+x_{(k-1) / 2}\right\}$ where, for $1 \leq i \leq(k-1) / 2, x_{i} \in\{1,2, \ldots,(v-k) / 2 k\}$. $B$ cannot have two (or more) elements from the same coset appearing as points, otherwise a difference that is a multiple of $v / k$ would be in $B$ and all such differences must be in the regular short orbit base block. Taking the elements of $B^{d}$ modulo $v / k$ yields the set $B_{m o d}^{d}=\left\{ \pm x_{1}, \pm x_{2} \ldots, \pm x_{(k-1) / 2}\right\}$. Each of the elements in $B_{m o d}^{d}$ represents a coset, and at most one representative of each coset may appear as a point in $B$. Since the number of non-zero points in $B$ is $k-1$ and $\left|B_{m o d}^{d}\right|=2 \cdot(k-1) / 2$, a point from each coset in $B^{d}$ must appear in $B$.

Let us take a closer look at $B_{m o d}^{d}$. We show that $B_{m o d}^{d} \cup\{0\}$ is a subgroup of $\mathbb{Z}_{v / k}$. Zero is in $B_{m o d}^{d} \cup\{0\}$ and for each $x \in B_{m o d}^{d}$ we can see that $-x \in B_{m o d}^{d}$. To see that $B_{m o d}^{d}$ is closed under addition, consider two elements, $x_{i}, x_{j} \in B_{m o d}^{d}$. Mapping these elements back to points in $B$, these elements have the form $x_{i}+r v / k, x_{j}+r^{\prime} v / k$, where $r, r^{\prime} \in\{0,1, \ldots,(k-1)\}$. Now $x_{j}-x_{i} \equiv x_{j}-x_{i}+\left(r^{\prime}-r\right) v / k(\bmod v / k)$, so an element of the coset $W \pm\left(x_{j}-x_{i}\right)$ appears as a difference of two points in 
$B$. Because all elements of a coset appear in exactly one base block $x_{j}-x_{i} \in B_{m o d}^{d}$. The modulo $v / k$ mapping can be applied to each of the full orbit base blocks in $S$; recall that we have assumed there exist at least two such base blocks in $S$. The result is a non-trivial collection of proper subgroups that together contain each element of $\mathbb{Z}_{v / k} \backslash\{0\}$ exactly once (and each subgroup contains zero). This is impossible since no proper subgroup can contain the generator 1.

Case 2: Suppose $\lambda>1$. There are three sub-cases to consider. First, suppose that all differences that are multiples of $v / k$ are in regular short orbit base blocks. This means that paired cosets of the form $W-d, W+d$ must pack perfectly into non-regular base blocks. Therefore, $2 k \lambda \mid 2\left(\begin{array}{l}k \\ 2\end{array}\right)$ must hold, which implies $2 \lambda \mid(k-1)$. Thus $k$ must be odd and, since $v / k$ is odd, $v$ must also be odd. Consider a nonregular base block $B$ and, without loss of generality, assume $0 \in B$. The collection of differences in $B$, denoted by $B^{d}$, contains $\lambda$ copies of each element of each coset that has a difference in $B . B$ cannot have two (or more) elements from any single coset appearing as points since the difference of these two points is a multiple of $v / k$ and such differences only appear in regular short orbit base blocks. Taking the elements of $B^{d}$ modulo $v / k$ yields the set $B_{m o d}^{d}=\left\{ \pm x_{1}, \pm x_{2} \ldots, \pm x_{(k-1) / 2}\right\}$. Each of the elements in $B_{\text {mod }}^{d}$ represents a coset, and at most one representative of each coset may appear as a point in $B$. Therefore, the maximum number of non-zero points in $B$ is $2 \cdot(k-1) / 2 \lambda<k-1$, which means we do not have enough points for block $B$. 
Second, suppose some of the differences $\{v / k, 2 v / k, \ldots,(k-1) v / k\}$ are not in regular short orbit base blocks. Further, suppose those differences that are not in regular short orbit base blocks are not all in the same non-regular base block. There exist at least two non-regular base blocks, say $B_{d}$ and $B_{f}$, containing differences $r v / k$ and $r^{\prime} v / k$, respectively, for $r, r^{\prime} \in\{1,2, \ldots,(k-1)\}$. Clearly, $r^{\prime} v / k-r v / k$ is a multiple of $v / k$ and we can select $r \neq r^{\prime}$ to ensure it is a non-zero multiple. To see that we can select $r \neq r^{\prime}$, note that each multiple of $v / k(\bmod v)$ appears $k$ times (but counts as only one appearance) in a regular short orbit base block and appears $\lambda$ times in the base blocks of the design. Since some of the differences in $\{v / k, 2 v / k, \ldots,(k-1) v / k\}$ are not in regular short orbit base blocks, we know that an equal number of each of these differences must appear in non-regular base blocks. As these differences do not all appear in the same non-regular base block, we can find two base blocks each containing a different multiple of $v / k$. We can assume $r v / k$ and $r^{\prime} v / k$ are points in the respective blocks (shifting the base blocks if necessary).

Finally, suppose some of the differences $\{v / k, 2 v / k, \ldots,(k-1) v / k\}$ are not in regular short orbit base blocks and that the differences not in a regular short orbit base block are all in the same non-regular base block. $S$ contains three types of base block: regular short orbit, non-regular (full or short orbit) containing differences that are multiples of $v / k$, and non-regular (full or short orbit) containing no differences that are multiples of $v / k$. Notice that this last type of base block is exactly the type 
of base block described in the first case, and therefore, these base blocks do not exist. This implies that $S$ must be generated by regular short orbit base blocks and a single non-regular base block; a contradiction to our assumption that $S$ contains at least two non-regular base blocks.

Lemma 4.2.22. Let $S$ be a cyclic $(v, k, \lambda)$-BIBD, $k \geq 3$, with a regular short orbit base block $B_{e}$. If $n_{3}$ is odd, $v / k$ is odd, and $n_{1}+n_{2} \geq 2$, then there exist nonregular base blocks $B_{d}=\left\{0, d_{1}, \ldots, d_{(k-1)}\right\}$ and $B_{f}=\left\{0, f_{1}, \ldots, f_{(k-1)}\right\}$, containing differences $d_{s}$ and $f_{t}$, respectively, such that $f_{t}-d_{s}=r v / k, r \in\{1, \ldots,(k-1)\}$. Let $U\left(B_{d}, d_{1}\right)=\left\{U_{0}, U_{1}, \ldots, U_{m-1}\right\}$ and $U\left(B_{f}, f_{1}\right)=\left\{Q_{0}, Q_{1}, \ldots, Q_{m^{\prime}-1}\right\}$ be collections of partial Ucycles representing the blocks of Dev $\left(B_{d}\right)$ and Dev $\left(B_{f}\right)$, respectively, with $d_{1} \neq d_{s}$ and $f_{1} \neq f_{t}$ used to form the sequences. The block $x+B_{e}, x \in\{0,1, \ldots$ $\ldots, v / k-1\}$, can be inserted between a partial Ucycle in $U\left(B_{d}, d_{1}\right)$ and a partial Ucycle in $U\left(B_{f}, f_{1}\right)$.

Proof. Let $S$ be a cyclic $(v, k, \lambda)$-BIBD, $k \geq 3$, with regular short orbit base block $B_{e}$. Suppose we wish to insert the block $x+B_{e}$, for some $x \in\{0,1, \ldots, v / k-1\}$, into existing partial Ucycles representing some of the blocks of $S$. Since $n_{3}$ is odd, and $v / k$ is odd, and the number of non-regular base blocks is at least two, Lemma 4.2.21 implies there exist two non-regular base blocks, $B_{d}=\left\{0, d_{1}, \ldots, d_{(k-1)}\right\}$ and $B_{f}=\left\{0, f_{1}, \ldots, f_{(k-1)}\right\}$, such that $f_{t}-d_{s}=r v / k, s, t \in\{2,3, \ldots, k-1\}$, for some 
$r \in\{1,2 \ldots,(k-1)\}$. Let $U\left(B_{d}, d_{1}\right)=\left\{U_{0}, U_{1}, \ldots, U_{m-1}\right\}$ be a collection of partial Ucycles representing $\operatorname{Dev}\left(B_{d}\right)$, with $d_{1} \neq d_{s}$ used to form the sequences. Let $U\left(B_{f}, f_{1}\right)=\left\{Q_{0}, Q_{1}, \ldots, Q_{m^{\prime}-1}\right\}$ be a collection of partial Ucycles representing the blocks of $\operatorname{Dev}\left(B_{f}\right)$, with $f_{1} \neq f_{t}$ used to form the sequences.

The block to be inserted, $x+B_{e}$, will be represented by the pair $\{x, x+r v / k\}$. To perform this insertion we must find $x-d_{s}$ in one of the partial Ucycles in $U\left(B_{d}, d_{1}\right)$. Note that a collection of partial Ucycles representing the blocks developed from a non-regular base block contains every element of $\mathbb{Z}_{n}$, where $n$ is the number of blocks generated by the non-regular base block. As a result, if the non-regular base block is short, $x-d_{s}$ may not appear in the collection of partial Ucycles. If $x-d_{s}$ does not appear, add $n$ to each point in $U\left(B_{d}, d_{1}\right) . U\left(B_{d}, d_{1}\right)+n$ represents the same blocks as $U\left(B_{d}, d_{1}\right)$ but now contains the points $\{n, n+1, \ldots, 2 n-1\}$. In fact, any one of the collections of partial Ucycles $U\left(B_{d}, d_{1}\right)+\ell n$, for $\ell \in\{0,1, \ldots,(w-1)\}$, where $w$ is the smallest value such that $w n \equiv 0(\bmod v)$, can be used to represent the blocks of $\operatorname{Dev}\left(B_{d}\right)$. As $\cup_{\ell=0}^{w-1}\left(U\left(B_{d}, d_{1}\right)+\ell n\right)=\mathbb{Z}_{v}$, there is a collection of partial Ucycles representing the blocks developed from the non-regular short orbit base block that contains the point $x-d_{s}$. In the case of a full orbit base block, the collection $U\left(B_{d}, d_{1}\right)$ contains every element of $\mathbb{Z}_{v}$, so $x-d_{s}$ must appear. We can determine an $a \in \mathbb{Z}_{v}$ and an $i \in \mathbb{N}$ such that $a d_{1}+\ell n+i=x-d_{s}, \ell \in\{0,1, \ldots,(w-1)\}$, where $\ell$ may be zero and $n=v$ if $B_{d}$ is a full orbit base block. By the same reasoning there must exist a 
$c \in \mathbb{Z}_{v}$ and a $j \in \mathbb{N}$ such that $c f_{1}+h \cdot m+j=a d_{1}+\ell \cdot n+i, h \in\left\{0,1, \ldots,\left(w^{\prime}-1\right)\right\}$ where $m=\left|\operatorname{Dev}\left(B_{f}\right)\right|$ and $w^{\prime}$ is the smallest value such that $w^{\prime} m \equiv 0(\bmod v)$. To simplify notation, let $I=\ell \cdot n+i$ and $J=h \cdot m+j$. We will insert $x+B_{e}$ between partial Ucycles $U_{I}$ and $Q_{J}$. Break $U_{I}$ between $(a-1) d_{1}+I$ and $a d_{1}+I$, then add $a d_{1}+I$ to the end of the segment $a d_{1}+I,(a+1) d_{1}+I, \ldots(a-1) d_{1}+I$ to obtain a segment starting and ending with $a d_{1}+I$. Break $Q_{J}$ between $(c-1) f_{1}+J$ and $c f_{1}+J$, then add $c f_{1}+J$ to the end of the segment $c f_{1}+J,(c+1) f_{1}+J, \ldots,(c-1) f_{1}+J$ to obtain a segment starting and ending with $c f_{1}+J$. Without loss of generality, select the second segment and reverse its orientation. Reattach the segments so that $\ldots,(a-1) d_{1}+I, a d_{1}+I$ is joined to $c f_{1}+J,(c-1) f_{1}+J, \ldots$ and such that $\ldots,(c+1) f_{1}+J, c f_{1}+J$ is joined to $a d_{1}+I,(a+1) d_{1}+I, \ldots$ The two segments now have opposite orientations, that is, if we pass along the sequence $U_{I}$ in the direction such that vertex labels increase by $+d_{1}$, then as we pass along $Q_{J}$ in the same direction the increments are $-f_{1}$. At the two joins identify the duplicate point $a d_{1}+I=c f_{1}+J$. Finally, take the segment $(c+1) f_{1}+J, a d_{1}+I,(a+1) d_{1}+I$ and replace it with $(c+1) f_{1}+J, c f_{1}+f_{t}+J, a d_{1}+d_{s}+I,(a+1) d_{1}+I$. The block represented by $\left\{a d_{1}+d_{s}+I, c f_{1}+f_{t}+J\right\}$ has been inserted. This pair of points is equal to $\left\{\left(x-d_{s}\right)+d_{s},\left(x-d_{s}\right)+f_{t}\right\}$ which is clearly equal to $\{x, x+r v / k\}$ and thus represents $x+B_{e}$. The blocks represented by the original partial Ucycles $U_{I}$ and $Q_{J}$ are intact, however, the block $I+\left\{a d_{1},(a+1) d_{1}, a d_{1}+d_{2}, \ldots, a d_{1}+d_{s}, \ldots, a d_{1}+d_{(k-1)}\right\}$ 
which was originally represented by the pair $\left\{a d_{1}+I,(a+1) d_{1}+I\right\}$ is now represented by $\left\{a d_{1}+d_{s}+I,(a+1) d_{1}+I\right\}$ and the block $J+\left\{c f_{1},(c+1) f_{1}, c f_{1}+f_{2}, \ldots, c f_{1}+\right.$ $\left.f_{t}, \ldots, c f_{1}+f_{(k-1)}\right\}$ which was originally represented by the pair $\left\{c f_{1}+J,(c+1) f_{1}+J\right\}$ is now represented by $\left\{c f_{1}+f_{t}+J,(c+1) f_{1}+J\right\}$.

This insertion is illustrated in Figure 23. Green lines and numbers represent changes to the original partial Ucycles - the green lines are new connections and the green numbers are altered numbers (with original and identified numbers in square brackets). The blue numbers and lines represent $U_{I}$ and the red letters and numbers represent $Q_{J}$. We will call this procedure Insertion $C$.

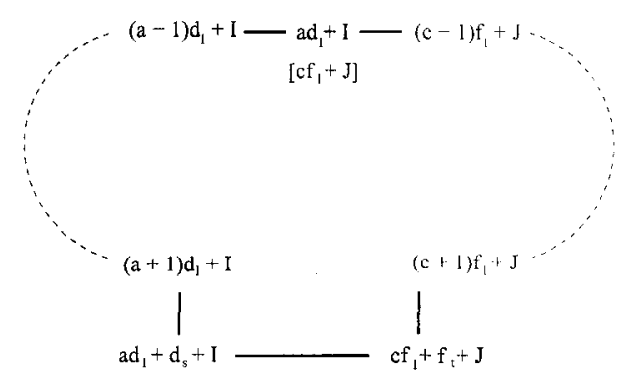

Figure 23: Insertion $C$ : a single block developed from a regular short orbit base block is inserted between two partial Ucycles from two different collections

The following corollary is the companion to Corollary 4.2 .16 for $n_{3}=1$.

Corollary 4.2.23. Let $S$ be a cyclic $(v, k, \lambda)$-BIBD, $k \geq 3$, with regular short orbit base block $B_{e}$ and $n_{3}=1$.

(i) If $v / k$ is even and $n_{2} \leq\lceil\lambda \phi(v / k) /(k-1)\rceil-1$, then there exists a collection of length- $\ell$ partial Ucycles representing the blocks developed from a full orbit base 
block into which all blocks of Dev $\left(B_{e}\right)$ can be inserted.

(ii) If $v / k$ is odd and $n_{2} \leq\lceil\lambda \phi(v / k) /(k-1)\rceil-3$, then there exists a collection of length- $\ell$ partial Ucycles representing the blocks developed from a full orbit base block, and two collections of length- $\ell$ partial Ucycles representing the blocks developed from two non-regular base blocks (distinct from the former) into which all blocks of $\operatorname{Dev}\left(B_{e}\right)$ can be inserted.

Proof. Let $S$ be a cyclic $(v, k, \lambda)$-BIBD, $k \geq 3$, with a regular short orbit base block $B_{e}$ and $n_{3}=1$. If $v / k$ is even, we need only apply Lemma 4.2 .19 with $x=1$. Note that since $v / k$ is even all blocks of $\operatorname{Dev}\left(B_{e}\right)$ can be paired and inserted into the available collection of length- $\ell$ partial Ucycles which represent the blocks developed from a full orbit base block.

If $v / k$ is odd we must apply Lemmas 4.2 .19 and 4.2 .22 in tandem. To ensure that the two non-regular base blocks required for a single insertion (Lemma 4.2.22) are not used for the insertion of paired blocks (Lemma 4.2.19) we select these blocks first, denote them $B_{2}$ and $B_{3}$, and then apply Lemma 4.2 .19 with $x=3$. This allows us to avoid blocks $B_{2}$ and $B_{3}$ when selecting a full orbit base block $B_{1}$ having a difference coprime to $v / k$. Insert all but one block of $\operatorname{Dev}\left(B_{e}\right)$ into the collection of length- $\ell$ partial Ucycles representing the blocks of $\operatorname{Dev}\left(B_{1}\right)$ (using the method described in Lemma 4.2.19), then apply Lemma 4.2 .22 with base blocks $B_{2}$ and $B_{3}$ to insert the remaining block of $\operatorname{Dev}\left(B_{e}\right)$. 
We now have all the insertion procedures necessary to insert regular short orbit blocks into collections of partial Ucycles. It remains to determine when all regular short orbit blocks in a design can be inserted into collections of partial Ucycles representing the non-regular blocks of the design. To minimize the number of collections of partial Ucycles required for insertion of regular short orbit blocks, execute as many insertions of the type described in Lemma 4.2 .15 as possible. That is, pair copies of the regular short orbit base block for insertion. All blocks developed from a pair of regular short orbit base blocks can be inserted into a collection of partial Ucycles representing the blocks generated by one full or one non-regular short orbit base block. If there are an odd number of regular short orbit base blocks in a design, $S$, before doing any insertions, identify the up to three collections of partial Ucycles that facilitate the insertion of blocks generated by a single regular short orbit base block (as per Corollary 4.2.23). If there are an odd number of regular short orbit base blocks in $S$ then a full orbit base block containing a difference coprime to $v / k$ is required. In addition, if $v / k$ is odd, then two non-regular base blocks (either full orbit or nonregular short orbit) having the property that the difference of an element in each of the base blocks is a multiple of $v / k$ are required. Lemma 4.2 .24 states conditions under which all regular short orbit blocks can be inserted into existing collections of partial Ucycles. 
Lemma 4.2.24. Let $S$ be a cyclic $(v, k, \lambda)-B I B D, k \geq 3$.

(i) If $n_{3}$ is even and $n_{1}+n_{2} \geq n_{3} / 2$, then all blocks generated by regular short orbit base blocks can be inserted into the collections of partial Ucycles representing the blocks developed from non-regular base blocks.

(ii) If $n_{3}$ is odd, $n_{1}+n_{2} \geq\left\lfloor n_{3} / 2\right\rfloor+3$ and $n_{2} \leq\lceil\lambda \phi(v / k) /(k-1)\rceil-3$, then all blocks generated by regular short orbit base blocks can be inserted into the collections of partial Ucycles representing the blocks developed from non-regular base blocks.

Proof. Let $S$ be a cyclic $(v, k, \lambda)$-BIBD, $k \geq 3$, and let $B_{e}$ denote the regular short orbit base block that may appear in $S$. If $n_{3}$ is even, then by $n_{3} / 2$ applications of Lemma 4.2.15, all the blocks developed from the $n_{3}$ regular short orbit base blocks can be inserted into $n_{3} / 2$ collections of partial Ucycles representing the blocks developed from non-regular base blocks.

If $n_{3}$ is odd and $n_{2} \leq\lceil\lambda \phi(v / k) /(k-1)\rceil-3$, Lemma 4.2 .23 can be applied to insert a single copy of $\operatorname{Dev}\left(B_{e}\right)$ into (at most) one collection of partial Ucycles representing a full orbit base block and two collections of partial Ucycles representing non-regular base blocks. Then $\left\lfloor n_{3} / 2\right\rfloor$ applications of Lemma 4.2 .15 can be used to insert the remaining $n_{3}-1$ copies of $B_{e}$ into $\left\lfloor n_{3} / 2\right\rfloor$ collections of partial Ucycles representing the blocks developed from non-regular base blocks.

The following corollary will be used to accommodate additional properties required 
in the proof of Theorem 4.2.3. From now on, we will assume we require full orbit base blocks with a difference coprime to $v$ (instead of $v / k$ ) for insertion of regular short orbit blocks. Furthermore, we will use the term special to refer to the insertion methods described in Lemmas 4.2.13 and 4.2.22 as these require extra properties of the collections of partial Ucycles into which insertions are made.

Corollary 4.2.25. Let $S$ be a cyclic $(v, k, \lambda)-B I B D, k \geq 3$. If $n_{1}+n_{2} \geq\left\lfloor n_{3} / 2\right\rfloor+3$ and $n_{2} \leq\lceil\lambda \phi(v) /(k(k-1))\rceil-4$, then all blocks generated by regular short orbit base blocks can be inserted into collections of partial Ucycles representing the blocks developed from non-regular base blocks and this can done such that a length-v partial Ucycle representing the blocks of a full orbit base block is not used for special insertions.

Proof. Let $S$ be a cyclic $(v, k, \lambda)$-BIBD, $k \geq 3$. Lemma 4.2 .24 implies that if $n_{1}+$ $n_{2} \geq\left\lfloor n_{3} / 2\right\rfloor+3$ and $n_{2} \leq\lceil\lambda \phi(v) /(k(k-1))\rceil-3$, all blocks generated by regular short orbit base blocks can be inserted into collections of partial Ucycles. As the minimum number of non-regular base blocks containing a difference coprime to $v$ is $[\lambda \phi(v) /(k(k-1))\rceil$, there exist at least four full orbit base blocks containing a difference coprime to $v$ when $n_{2} \leq\lceil\lambda \phi(v) /(k(k-1))\rceil-4$. At most three of these base blocks will be needed for insertion of regular short orbit blocks. Using a difference coprime to $v$ to generate a partial Ucycle for the blocks of a full orbit base block results in a length- $v$ Ucycle containing each element of $\mathbb{Z}_{v}$ exactly once.

We now have all the tools required to prove Theorem 4.2.3: Let $S$ be a cyclic 
$(v, k, \lambda)$-BIBD with $k \geq 3$. If $n_{2} \leq\lceil\lambda \phi(v) /(k(k-1))\rceil-4$ and $n_{1}+n_{2} \geq\left\lfloor n_{3} / 2\right\rfloor+3$, then $S$ admits a Ucycle of rank two.

Proof of Theorem 4.2.3. Let $S$ be a cyclic $(v, k, \lambda)$-BIBD, $k \geq 3$, with the required properties. Corollary 4.2.25 implies that all blocks generated by regular short orbit base blocks can be inserted into collections of partial Ucycles representing blocks developed from non-regular base blocks. Furthermore, the special insertions can be executed while avoiding a length- $v$ partial Ucycle representing the blocks of a full orbit base block.

Specifically, to construct a Ucycle for the blocks of $S$, first determine the parity of the number of regular short orbit base blocks in $S$. If the number of regular short orbit base blocks is odd and $v / k$ is odd, begin by selecting a pair of non-regular short orbit base blocks satisfying the requirements of Lemma 4.2.22. Next, select a full orbit base block containing a difference coprime to $v$. Every cyclic $(v, k, \lambda)$ BIBD with $n_{2} \leq\lceil\lambda \phi(v) /(k(k-1))\rceil-4$ contains such a block. After selecting the three special base blocks, there remains at least one full orbit base block containing a difference coprime to $v$. Denote this block $B_{p}$. Construct collections of partial Ucycles for each non-regular base block in $S$ using the methods described in Lemmas 4.2.4, 4.2.5, 4.2.10 and 4.2.11. Take into account that constructing the collections of partial Ucycles representing the blocks developed from the three special base blocks described above requires extra care in the selection of the difference used for development. 
Furthermore, develop $B_{p}$ by a difference coprime to $v$ to create a length- $v$ partial Ucycle; denote this partial Ucycle $U\left(B_{p}, p_{1}\right)$. Once the collections of partial Ucycles have been created, insert the blocks developed from pairs of regular short orbit base blocks using the method described in Lemma 4.2.15, but do not use the collections of partial Ucycles representing the three special base blocks. Note that we can use the partial Ucycle $U\left(B_{p}, p_{1}\right)$. Finally, if a single regular short orbit base block remains, use the set aside collections of partial Ucycles and apply Lemma 4.2.19, and possibly Lemma 4.2.22. At this juncture, all blocks of $S$ are represented in partial Ucycles and each partial Ucycle is of length at least two. It remains to show that these partial Ucycles can be joined together to form a Ucycle for the blocks of $S$.

The method for joining together two partial Ucycles is as follows. Suppose we have two partial Ucycles $U\left(B_{d}, d_{1}\right)$ and $U\left(B_{f}, f_{1}\right)$, both containing a point $x \in \mathbb{Z}_{v}$. Convert each partial Ucycle to a path having $x$ as one endpoint by breaking one of the edges adjacent to $x$. Suppose we break $U\left(B_{d}, d_{1}\right)$ such that the endpoints of the segment are $x$ and $y$ and suppose we break $U\left(B_{f}, f_{1}\right)$ such that the endpoints of the segment are $x$ and $z$. Join these two segments together by joining the $x$ in $U\left(B_{d}, d_{1}\right)$ to the $z$ in $U\left(B_{f}, f_{1}\right)$ and joining the $x$ in $U\left(B_{f}, f_{1}\right)$ to the $y$ in $U\left(B_{d}, d_{1}\right)$. The blocks that were represented by $U\left(B_{d}, d_{1}\right)$ and $U\left(B_{f}, f_{1}\right)$ are intact and there is no possibility of creating a 3-claw with this join procedure.

Each collection of partial Ucycles representing a full orbit base block contains 
every element of $\mathbb{Z}_{v}$ exactly once (refer to Lemmas 4.2 .4 and 4.2.5). Since $U\left(B_{p}, p_{1}\right)$ is a length- $v$ partial Ucycle (representing a full orbit base block), $U\left(B_{p}, p_{1}\right)$ contains every element of $\mathbb{Z}_{v}$, and therefore, every partial Ucycle shares at least one point with $U\left(B_{p}, p_{1}\right)$. To create a Ucycle representing all blocks of $S$, we grow the partial Ucycle $U\left(B_{p}, p_{1}\right)$ by applying the above join procedure with $U\left(B_{p}, p_{1}\right)$ and another partial Ucycle not yet joined to $U\left(B_{p}, p_{1}\right)$, until no such partial Ucycles exist.

We note that the properties $n_{1}+n_{2} \geq\left\lfloor n_{3} / 2\right\rfloor+3$ and $n_{2} \leq\lceil\lambda \phi(v) /(k(k-1))\rceil-4$, required by Theorem 4.2.3, account for the most degenerate cyclic BIBDs. In most cases these inequalities far exceed the actual number of full orbit base blocks required for creation of a rank two Ucycle. We now focus on several specific families for which the inequalities of Theorem 4.2 .3 can be greatly refined. All specific values of the Euler totient function used in the following corollaries were obtained from [52]. We begin with triple systems.

Corollary 4.2.26. Every cyclic $T S(v, \lambda)$, with $v \geq 7$, admits a Ucycle of rank two. Proof. Suppose $S$ is a cyclic $\operatorname{TS}(v, \lambda)$. As non-regular short orbit base blocks do not exist in triple systems (i.e. $n_{2}=0$ ), all regular short orbit blocks in $S$ must be inserted into collections of partial Ucycles representing the blocks developed from full orbit base blocks. The number of blocks in $S$ is $(v(v-1) \lambda) / 6$. Each full orbit base block generates $v$ of these blocks, while each regular short orbit base block generates 
$v / 3$ of these blocks, therefore,

$$
\frac{v(v-1) \lambda}{6}=v n_{1}+\frac{v}{3} n_{3}
$$

which implies that the number of full orbit base blocks used to generate $S$ is

$$
n_{1}=\frac{(v-1) \lambda}{6}-\frac{n_{3}}{3}
$$

As $n_{3} \leq \lambda, n_{1} \geq(v-3) \lambda / 6$. We now divide our analysis into three cases.

Case 1: Suppose $n_{3}$ is even. Lemma 4.2 .24 implies that if $n_{1} \geq n_{3} / 2$, all regular short orbit base blocks can be inserted into collections of partial Ucycles representing full orbit blocks. Since $n_{1} \geq(v-3) \lambda / 6$ and $n_{3} \leq \lambda, n_{1} \geq n_{3} / 2$ holds for all triple systems with $(v-3) \lambda / 6 \geq \lambda / 2$. Simplifying this expression yields $(v-6) \lambda \geq 0$. Therefore, when $v \geq 6$, all regular short orbit blocks can be inserted into collections of partial Ucycles. Furthermore, when $v \geq 6$, the element $1 \in \mathbb{Z}_{v}$ must appear as a difference in a full orbit base block so there exists a length- $v$ partial Ucycle to which all other partial Ucycles can be joined. We conclude that for this case, every cyclic $\operatorname{TS}(v, \lambda), v \geq 6$, admits a rank two Ucycle.

Case 2: Suppose $n_{3}$ is odd and $v / 3$ is even. To create a Ucycle for the blocks of $S$, we require the existence of two full orbit base blocks each containing a difference coprime to $v$, or one full orbit base block with all of its differences coprime to $v$. In the first case, one block will be used in the application of Lemma 4.2 .19 and the other will be used to create a length- $v$ partial Ucycle to which all other partial Ucycles can 
be joined. In the second case, note that one of the differences coprime to $v$ can be used to create a length- $v$ partial Ucycle and there will be another difference coprime to $v$ in the block that can be used in the application of Lemma 4.2.19. The minimum number of full orbit base blocks containing differences coprime to $v$ is $\lceil\lambda \phi(v) / 6\rceil$, so we require $\lambda \phi(v) / 6 \geq 1$ to ensure the existence of a length- $v$ partial Ucycle. This inequality holds for all $v \geq 13$.

Insertion of regular short orbit blocks proceeds as follows. First insert a single copy of $\operatorname{Dev}(\{0, v / 3,2 v / 3\})$ into a collection of partial Ucycles representing a full orbit base block containing a difference coprime to $v$ (using the method described in Lemma 4.2.19). Second, insert the remaining $n_{3}-1$ copies of $\operatorname{Dev}(\{0, v / 3,2 v / 3\})$ into $\left(n_{3}-1\right) / 2$ other full orbit base blocks. This is done via $\left(n_{3}-1\right) / 2$ applications of Lemma 4.2.15. In total, insertions of regular short orbit blocks require $\left(n_{3}-1\right) / 2+1$ distinct full orbit base blocks. Since $n_{1} \geq(v-3) \lambda / 6$ and $n_{3} \leq \lambda$, we will have sufficient full orbit base blocks when $(v-3) \lambda / 6 \geq(\lambda-1) / 2+1$. This inequality simplifies to $(v-6) \lambda \geq 3$, therefore, sufficient full orbit base blocks exist for all $v \geq 9$.

We now deal with the values of $v(7 \leq v \leq 12)$ not covered by the general argument regarding existence of two full orbit base blocks each containing a difference coprime to $v$. Since $v / 3$ is even, the only value to consider is twelve. The smallest index for which a cyclic $\operatorname{TS}(12, \lambda)$ exists is two [19]. Each of the integers $1,5,7,11$ is coprime to twelve, and for $\lambda \geq 2$, the $\lambda$ copies of each of these integers cannot all appear as 
differences in the same full orbit base block, therefore, there exist at least two full orbit base blocks each containing a difference coprime to twelve. We conclude that for this case, every cyclic $\operatorname{TS}(v, \lambda), v \geq 7$, admits a rank two Ucycle.

Case 3: Suppose $n_{3}$ is odd and $v / 3$ is odd. To create a Ucycle for the blocks of $S$, we require the existence of four full orbit base blocks each containing a difference coprime to $v$, or three full orbit base blocks where one of these base blocks has all differences coprime to $v$. In the first case, the four blocks ensure that the selection of two blocks for use in the single block insertion (as described in Lemma 4.2.22) leaves us with at least two blocks each containing at least one difference coprime to $v$. One of these blocks will be used in the application of Lemma 4.2.19 and the other block will be used to create a length- $v$ partial Ucycle to which all other partial Ucycles can be joined. In the second case, the full orbit base block with all differences coprime to $v$ can be developed by a difference coprime to $v$ (yielding a length- $v$ partial Ucycle) while still fulfilling its other role (either in Lemma 4.2 .19 or 4.2.22). The minimum number of full orbit base blocks containing differences coprime to $v$ is $\lceil\lambda \phi(v) / 6\rceil$, so we require $\lambda \phi(v) / 6>2$ (which actually leaves two full orbit base blocks all of whose differences must be coprime to $v$ ), to ensure existence of these blocks. This inequality holds for all $v \geq 44$.

Insertion of regular short orbit blocks proceeds as follows. First insert a single copy of $\operatorname{Dev}(\{0, v / 3,2 v / 3\})$, less one block, into a collection of partial Ucycles representing 
a full orbit base block containing a difference coprime to $v$ (using the method described in Lemma 4.2.19). Second, insert the block leftover between two collections of partial Ucycles (using the method described in Lemma 4.2.22). Third, insert the remaining $n_{3}-1$ copies of $\operatorname{Dev}(\{0, v / 3,2 v / 3\})$ (this is done via $\left(n_{3}-1\right) / 2$ applications of Lemma 4.2.15) into $\left(n_{3}-1\right) / 2$ other full orbit base blocks. In total, insertions of regular short orbit blocks require $\left(n_{3}-1\right) / 2+3$ distinct full orbit base blocks. Since $n_{1} \geq(v-3) \lambda / 6$ and $n_{3} \leq \lambda$, we will have sufficient full orbit base blocks when $(v-3) \lambda / 6 \geq(\lambda-$ 1) $/ 2+3$. This inequality simplifies to $(v-6) \lambda \geq 15$, therefore, sufficient full orbit base blocks exist for all $v \geq 21$.

We now deal with the values of $v(7 \leq v \leq 43)$ not covered by the general arguments above. Since $v / 3$ is odd, the only values of $v$ to consider are $9,15,21,27,33$. For $v \geq 21$ only the condition on the number of full orbit base blocks containing a difference coprime to $v$ must be checked. That is, we wish to determine when $\lambda \phi(v) / 6>2$. As there exist Steiner triple systems for $v=21,27,33$, we must determine if $\phi(v)>12$ for each of these specific values of $v$. The inequality holds for $v=27,33$. When $v=21$, note that for $\lambda \geq 2, \lambda \phi(v) / 6>2$ holds. Therefore, it remains to prove that every cyclic STS(21) admits a Ucycle of rank two. We know that every cyclic STS(21) contains a regular short orbit base block (see Lemma 2.1.7) and three full orbit base blocks, so the minimal requirement of Theorem 4.2 .3 for the number of full orbit base blocks is not satisfied. However, despite this fact, our 
construction method can be used to construct Ucycles for each of the nonisomorphic cyclic STS(21)s. Each of the STS(21)s contains a full orbit base block with two differences coprime to 21 , so we can construct a length-21 partial Ucycle (to which all others can be joined) and also insert pairs of short orbit blocks into this partial Ucycle. The remaining short orbit block can be inserted between collections of partial Ucycles representing the blocks developed from the two other full orbit base blocks. Ucycles for the seven nonisomorphic cyclic STS(21)s (listed in [21]) are given in Tables 17, $18,19,20$.

For $v=9,15$, we must determine when there exist enough full orbit base blocks for (1) the insertion of all regular short orbit blocks, and (2) the creation of a length- $v$ partial Ucycle to which all other partial Ucycles can be joined. That is, we wish to determine when both $(v-6) \lambda \geq 15$ and $\lambda \phi(v) / 6>2$ hold. For $v=9$, the smallest index for which cyclic triple systems exist is three [19] and since $\phi(9)=6$, both inequalities hold for all $\operatorname{TS}(9, \lambda) \mathrm{s}$. For $v=15$, both inequalities hold for all $\lambda \geq 2$. It remains to show that the two nonisomorphic cyclic STS(15)s admit rank two Ucycles. These are given in Table 21.

We conclude that every cyclic $\operatorname{TS}(v, \lambda), v \geq 7$, admits a rank two Ucycle. 


\begin{tabular}{|c|c|c|c|c|c|}
\hline \multicolumn{3}{|c|}{ Base Blocks } & \multicolumn{3}{|c|}{ Base Blocks } \\
\hline $\multicolumn{3}{|c|}{0,1,3},\{0,4,12\},\{0,5,11\},\{0,7,14\}$ & $\multicolumn{3}{|c|}{0,1,3},\{0,4,12\},\{0,5,15\},\{0,7,14\}$ \\
\hline \multicolumn{3}{|c|}{ Ucycle } & \multicolumn{3}{|c|}{ Ucycle } \\
\hline \multicolumn{3}{|c|}{$\begin{array}{c}0,7,6,8,1,20,13,12,11,2,14,5 \\
17,8,20,11,10,1,13,4,16,7,19, \\
10,9,8,10,3,1,2,18,13,8,3,19 \\
14,9,4,20,15,10,5,11,4,12,3,15 \\
6,18,9,0,16,11,6,1,17,12,7,2,3 \\
4,5,19,18,17,16,15,14,16,2\end{array}$} & \multicolumn{3}{|c|}{$\begin{array}{c}0,7,6,8,1,20,13,12,11,17,2,8, \\
14,4,11,3,7,11,15,19,2,6,10,14 \\
18,1,5,9,13,17,0,4,8,12,16,20 \\
5,11,10,9,3,18,12,6,0,15,9,8,10 \\
3,1,16,10,4,19,13,7,1,2,3,4,5 \\
19,18,17,16,15,14,16,2\end{array}$} \\
\hline \multicolumn{3}{|c|}{$\begin{array}{l}\text { Blocks Represented by Ucycle } \\
\text { (read column-wise) }\end{array}$} & \multicolumn{3}{|c|}{$\begin{array}{l}\text { Blocks Represented by Ucycle } \\
\text { (read column-wise) }\end{array}$} \\
\hline$\{0,7,14\}$ & $\{8,9,11\}$ & $\{18,1,9\}$ & $\{0,7,14\}$ & $\{18,1,9\}$ & $\{3,10,17\}$ \\
\hline$\{6,7,9\}$ & $\{7,8,10\}$ & $\{9,13,0\}$ & $\{6,7,9\}$ & $\{1,5,13\}$ & $\{0,1,3\}$ \\
\hline$\{5,6,8\}$ & $\{3,10,17\}$ & $\{16,0,6\}$ & $\{5,6,8\}$ & $\{5,9,17\}$ & $\{1,6,16\}$ \\
\hline$\{1,8,15\}$ & $\{0,1,3\}$ & $\{11,16,1\}$ & $\{1,8,15\}$ & $\{9,13,0\}$ & $\{16,0,10\}$ \\
\hline$\{19,20,1\}$ & $\{1,2,4\}$ & $\{6,11,17\}$ & $\{19,20,1\}$ & $\{13,17,4\}$ & $\{10,15,4\}$ \\
\hline$\{6,13,20\}$ & $\{18,2,8\}$ & $\{1,6,12\}$ & $\{6,13,20\}$ & $\{17,0,8\}$ & $\{4,9,19\}$ \\
\hline$\{12,13,15\}$ & $\{13,18,3\}$ & $\{17,1,7\}$ & $\{12,13,15\}$ & $\{0,4,12\}$ & $\{19,3,13\}$ \\
\hline$\{11,12,14\}$ & $\{8,13,19\}$ & $\{12,17,2\}$ & $\{11,12,14\}$ & $\{4,8,16\}$ & $\{13,18,7\}$ \\
\hline$\{11,15,2\}$ & $\{3,8,14\}$ & $\{7,12,18\}$ & $\{17,1,11\}$ & $\{8,12,20\}$ & $\{7,12,1\}$ \\
\hline$\{2,6,14\}$ & $\{19,3,9\}$ & $\{2,7,13\}$ & $\{2,7,17\}$ & $\{12,16,3\}$ & $\{1,2,4\}$ \\
\hline$\{14,18,5\}$ & $\{14,19,4\}$ & $\{2,3,5\}$ & $\{8,13,2\}$ & $\{16,20,7\}$ & $\{2,3,4\}$ \\
\hline$\{5,9,17\}$ & $\{9,14,20\}$ & $\{3,4,6\}$ & $\{14,19,8\}$ & $\{5,10,20\}$ & $\{3,4,6\}$ \\
\hline$\{17,0,8\}$ & $\{4,9,15\}$ & $\{4,5,7\}$ & $\{20,4,14\}$ & $\{11,16,5\}$ & $\{4,5,7\}$ \\
\hline$\{8,12,20\}$ & $\{20,4,10\}$ & $\{5,12,19\}$ & $\{4,11,18\}$ & $\{10,11,13\}$ & $\{5,12,19\}$ \\
\hline$\{20,3,11\}$ & $\{15,20,5\}$ & $\{18,19,0\}$ & $\{20,3,11\}$ & $\{9,10,12\}$ & $\{18,19,0\}$ \\
\hline$\{10,11,13\}$ & $\{10,15,0\}$ & $\{17,18,20\}$ & $\{3,7,15\}$ & $\{9,14,3\}$ & $\{17,18,20\}$ \\
\hline$\{10,14,1\}$ & $\{5,10,16\}$ & $\{16,17,19\}$ & $\{7,11,19\}$ & $\{3,8,18\}$ & $\{16,17,19\}$ \\
\hline$\{1,5,13\}$ & $\{0,5,11\}$ & $\{15,16,18\}$ & $\{11,15,2\}$ & $\{18,2,12\}$ & $\{15,16,18\}$ \\
\hline$\{13,17,4\}$ & $\{4,11,18\}$ & $\{14,15,17\}$ & $\{15,19,6\}$ & $\{12,17,6\}$ & $\{14,15,17\}$ \\
\hline$\{4,8,16\}$ & $\{0,4,12\}$ & $\{13,14,16\}$ & $\{19,2,10\}$ & $\{6,11,0\}$ & $\{13,14,16\}$ \\
\hline$\{16,20,7\}$ & $\{12,16,3\}$ & $\{2,9,16\}$ & $\{2,6,14\}$ & $\{0,5,15\}$ & $\{2,9,16\}$ \\
\hline$\{7,11,19\}$ & $\{3,7,15\}$ & $\{20,0,2\}$ & $\{6,10,18\}$ & $\{15,20,9\}$ & $\{20,0,2\}$ \\
\hline$\{19,2,10\}$ & $\{15,19,6\}$ & & $\{10,14,1\}$ & $\{8,9,11\}$ & \\
\hline$\{9,10,12\}$ & $\{6,10,18\}$ & & $\{14,18,5\}$ & $\{7,8,10\}$ & \\
\hline
\end{tabular}

Table 17: Ucycles of rank two for two of the seven nonisomorphic cyclic STS(21)s 


\begin{tabular}{|c|c|c|c|c|c|}
\hline \multicolumn{3}{|c|}{ Base Blocks } & \multicolumn{3}{|c|}{ Base Blocks } \\
\hline $\multicolumn{3}{|c|}{0,1,5},\{0,2,10\},\{0,3,9\},\{0,7,14\}$ & $\multicolumn{3}{|c|}{0,1,5},\{0,2,10\},\{0,3,15\},\{0,7,14\}$ \\
\hline \multicolumn{3}{|c|}{ Ucycle } & \multicolumn{3}{|c|}{ Ucycle } \\
\hline \multirow{6}{*}{\multicolumn{3}{|c|}{$\begin{array}{c}0,7,11,18,14,17,20,2,5,8,11,14 \\
15,16,17,10,9,8,12,5,1,4,7,10 \\
13,16,19,1,2,3,4,14,3,13,2,12 \\
1,11,0,18,15,12,9,6,3,9,2,10 \\
20,9,19,8,18,7,17,6,16,5,15,4 \\
5,6,13,12,11,15,1,18,19,20\end{array}$}} & \multirow{6}{*}{\multicolumn{3}{|c|}{$\begin{array}{c}0,7,11,18,14,16,18,20,5,11,17 \\
2,8,14,2,9,1,3,5,7,9,11,13,15 \\
17,19,0,2,4,6,8,10,12,14,15, \\
16,17,10,9,3,18,12,6,0,15,9 \\
8,12,5,1,16,10,4,19,13,7,1,2 \\
3,4,5,6,13,12,11,15,1,18,19,20\end{array}$}} \\
\hline & & & & & \\
\hline & & & & & \\
\hline & & & & & \\
\hline & & & & & \\
\hline & & & & & \\
\hline \multicolumn{3}{|c|}{$\begin{array}{l}\text { Blocks Represented by Ucycle } \\
\text { (read column-wise) }\end{array}$} & \multicolumn{3}{|c|}{$\begin{array}{l}\text { Blocks Represented by Ucycle } \\
\text { (read column-wise) }\end{array}$} \\
\hline$\{0,7,14\}$ & $\{13,16,1\}$ & $\{20,1,9\}$ & $\{0,7,14\}$ & $\{17,19,6\}$ & $\{0,1,5\}$ \\
\hline$\{6,7,11\}$ & $\{16,19,4\}$ & $\{9,11,19\}$ & $\{6,7,11\}$ & $\{19,0,8\}$ & $\{1,4,16\}$ \\
\hline$\{4,11,18\}$ & $\{19,1,7\}$ & $\{19,0,8\}$ & $\{4,11,18\}$ & $\{0,2,10\}$ & $\{16,19,10\}$ \\
\hline$\{13,14,18\}$ & $\{1,2,6\}$ & $\{8,10,18\}$ & $\{13,14,18\}$ & $\{2,4,12\}$ & $\{10,13,4\}$ \\
\hline$\{14,17,2\}$ & $\{2,3,7\}$ & $\{18,20,7\}$ & $\{14,16,3\}$ & $\{4,6,14\}$ & $\{4,7,19\}$ \\
\hline$\{17,20,5\}$ & $\{3,4,8\}$ & $\{7,9,17\}$ & $\{16,18,5\}$ & $\{6,8,16\}$ & $\{19,1,13\}$ \\
\hline$\{20,2,8\}$ & $\{4,6,14\}$ & $\{17,19,6\}$ & $\{18,20,7\}$ & $\{8,10,18\}$ & $\{13,16,7\}$ \\
\hline$\{2,5,11\}$ & $\{14,16,3\}$ & $\{6,8,16\}$ & $\{5,8,20\}$ & $\{10,12,20\}$ & $\{7,10,1\}$ \\
\hline$\{5,8,14\}$ & $\{3,5,13\}$ & $\{16,18,5\}$ & $\{11,14,5\}$ & $\{12,14,1\}$ & $\{1,2,6\}$ \\
\hline$\{8,11,17\}$ & $\{13,15,2\}$ & $\{5,7,15\}$ & $\{17,20,11\}$ & $\{14,15,19\}$ & $\{2,3,7\}$ \\
\hline$\{11,14,20\}$ & $\{2,4,12\}$ & $\{15,17,4\}$ & $\{2,5,17\}$ & $\{15,16,20\}$ & $\{3,4,8\}$ \\
\hline$\{14,15,19\}$ & $\{12,14,1\}$ & $\{4,5,9\}$ & $\{8,11,2\}$ & $\{16,17,0\}$ & $\{4,5,9\}$ \\
\hline$\{15,16,20\}$ & $\{1,3,11\}$ & $\{5,6,10\}$ & $\{14,17,8\}$ & $\{3,10,17\}$ & $\{5,6,10\}$ \\
\hline$\{16,17,0\}$ & $\{11,13,0\}$ & $\{6,13,20\}$ & $\{20,2,14\}$ & $\{9,10,14\}$ & $\{6,13,20\}$ \\
\hline$\{3,10,17\}$ & $\{18,0,6\}$ & $\{12,13,17\}$ & $\{2,9,16\}$ & $\{9,12,3\}$ & $\{12,13,17\}$ \\
\hline$\{9,10,14\}$ & $\{15,18,3\}$ & $\{11,12,16\}$ & $\{20,1,9\}$ & $\{3,6,18\}$ & $\{11,12,16\}$ \\
\hline$\{8,9,13\}$ & $\{12,15,0\}$ & $\{10,11,15\}$ & $\{1,3,11\}$ & $\{18,0,12\}$ & $\{10,11,15\}$ \\
\hline$\{7,8,12\}$ & $\{9,12,18\}$ & $\{1,8,15\}$ & $\{3,5,13\}$ & $\{12,15,6\}$ & $\{1,8,15\}$ \\
\hline$\{5,12,19\}$ & $\{6,9,15\}$ & $\{17,18,1\}$ & $\{5,7,15\}$ & $\{6,9,0\}$ & $\{17,18,1\}$ \\
\hline$\{0,1,5\}$ & $\{3,6,12\}$ & $\{18,19,2\}$ & $\{7,9,17\}$ & $\{0,3,15\}$ & $\{18,19,2\}$ \\
\hline$\{1,4,10\}$ & $\{0,3,9\}$ & $\{19,20,3\}$ & $\{9,11,19\}$ & $\{15,18,9\}$ & $\{19,20,3\}$ \\
\hline$\{4,7,13\}$ & $\{2,9,16\}$ & $\{20,0,4\}$ & $\{11,13,0\}$ & $\{8,9,13\}$ & $\{20,0,4\}$ \\
\hline$\{7,10,16\}$ & $\{0,2,10\}$ & & $\{13,15,2\}$ & $\{7,8,12\}$ & \\
\hline$\{10,13,19\}$ & $\{10,12,20\}$ & & $\{15,17,4\}$ & $\{5,12,19\}$ & \\
\hline
\end{tabular}

Table 18: Ucycles of rank two for two of the seven nonisomorphic cyclic STS(21)s 


\begin{tabular}{|c|c|c|c|c|c|}
\hline \multicolumn{3}{|c|}{ Base Blocks } & \multicolumn{3}{|c|}{ Base Blocks } \\
\hline $\multicolumn{3}{|c|}{0,1,5},\{0,2,13\},\{0,3,9\},\{0,7,14\}$ & $\multicolumn{3}{|c|}{0,1,9},\{0,2,5\},\{0,4,10\},\{0,7,14\}$ \\
\hline \multicolumn{3}{|c|}{ Ucycle } & \multicolumn{3}{|c|}{ Ucycle } \\
\hline \multicolumn{3}{|c|}{$\begin{array}{c}0,7,11,18,14,15,16,19,1,4,7,10 \\
13,16,17,10,9,8,12,5,1,2,5,8 \\
11,14,17,20,2,3,4,17,9,1,14,6 \\
19,11,3,16,8,0,18,15,12,9,6,3 \\
9,2,13,5,18,10,2,15,7,20,12,4 \\
5,6,13,12,11,15,1,18,19,20\end{array}$} & \multicolumn{3}{|c|}{$\begin{array}{l}0,7,3,20,16,1,15,9,13,6,2,19, \\
20,0,1,2,18,13,8,3,19,14,9,4, \\
20,15,10,5,0,16,11,6,1,17,12, \\
7,4,11,3,4,5,6,7,8,9,10,11,12, \\
13,14,15,16,17,18,19,15,11,17 \\
10,4,8,12,5,1,18,14,10,16,2,17\end{array}$} \\
\hline \multicolumn{3}{|c|}{$\begin{array}{l}\text { Blocks Represented by Ucycle } \\
\text { (read column-wise) }\end{array}$} & \multicolumn{3}{|c|}{$\begin{array}{l}\text { Blocks Represented by Ucycle } \\
\text { (read column-wise) }\end{array}$} \\
\hline$\{0,7,14\}$ & $\{11,14,20\}$ & $\{2,9,16\}$ & $\{0,7,14\}$ & $\{15,17,20\}$ & $\{13,14,1\}$ \\
\hline$\{6,7,11\}$ & $\{14,17,2\}$ & $\{0,2,13\}$ & $\{3,7,13\}$ & $\{10,12,15\}$ & $\{14,15,2\}$ \\
\hline$\{4,11,18\}$ & $\{17,20,5\}$ & $\{13,15,5\}$ & $\{20,3,9\}$ & $\{5,7,10\}$ & $\{15,16,3\}$ \\
\hline$\{13,14,18\}$ & $\{20,2,8\}$ & $\{5,7,18\}$ & $\{16,20,5\}$ & $\{0,2,5\}$ & $\{16,17,4\}$ \\
\hline$\{14,15,19\}$ & $\{2,3,7\}$ & $\{18,20,10\}$ & $\{12,16,1\}$ & $\{16,18,0\}$ & $\{17,18,5\}$ \\
\hline$\{15,16,20\}$ & $\{3,4,8\}$ & $\{10,12,2\}$ & $\{1,8,15\}$ & $\{11,13,16\}$ & $\{18,19,6\}$ \\
\hline$\{16,19,4\}$ & $\{4,6,17\}$ & $\{2,4,15\}$ & $\{5,9,15\}$ & $\{6,8,11\}$ & $\{15,19,4\}$ \\
\hline$\{19,1,7\}$ & $\{17,19,9\}$ & $\{15,17,7\}$ & $\{9,13,19\}$ & $\{1,3,6\}$ & $\{11,15,0\}$ \\
\hline$\{1,4,10\}$ & $\{9,11,1\}$ & $\{7,9,20\}$ & $\{6,13,20\}$ & $\{17,19,1\}$ & $\{7,11,17\}$ \\
\hline$\{4,7,13\}$ & $\{1,3,14\}$ & $\{20,1,12\}$ & $\{2,6,12\}$ & $\{12,14,17\}$ & $\{3,10,17\}$ \\
\hline$\{7,10,16\}$ & $\{14,16,6\}$ & $\{12,14,4\}$ & $\{19,2,8\}$ & $\{7,9,12\}$ & $\{0,4,10\}$ \\
\hline$\{10,13,19\}$ & $\{6,8,19\}$ & $\{4,5,9\}$ & $\{19,20,7\}$ & $\{2,4,7\}$ & $\{4,8,14\}$ \\
\hline$\{13,16,1\}$ & $\{19,0,11\}$ & $\{5,6,10\}$ & $\{20,0,8\}$ & $\{4,11,18\}$ & $\{8,12,18\}$ \\
\hline$\{16,17,0\}$ & $\{11,13,3\}$ & $\{6,13,20\}$ & $\{0,1,9\}$ & $\{2,3,11\}$ & $\{5,12,19\}$ \\
\hline$\{3,10,17\}$ & $\{3,5,16\}$ & $\{12,13,17\}$ & $\{1,2,10\}$ & $\{3,4,12\}$ & $\{1,5,11\}$ \\
\hline$\{9,10,14\}$ & $\{16,18,8\}$ & $\{11,12,16\}$ & $\{18,20,2\}$ & $\{4,5,13\}$ & $\{18,1,7\}$ \\
\hline, 13$\}$ & $\{8,10,0\}$ & $\{10,11,15\}$ & $\{13,15,18\}$ & $\{5,6,14\}$ & $\{14,18,3\}$ \\
\hline$\{7,8,12\}$ & $\{18,0,6\}$ & $\{1,8,15\}$ & $\{8,10,13\}$ & $\{6,7,15\}$ & $\{10,14,20\}$ \\
\hline$\{5,12,19\}$ & $\{15,18,3\}$ & $\{17,18,1\}$ & $\{3,5,8\}$ & $\{7,8,16\}$ & $\{6,10,16\}$ \\
\hline$\{0,1,5\}$ & $\{12,15,0\}$ & $\{18,19,2\}$ & $\{19,0,3\}$ & $\{8,9,17\}$ & $\{2,9,16\}$ \\
\hline$\{1,2,6\}$ & $\{9,12,18\}$ & $\{19,20,3\}$ & $\{14,16,19\}$ & $\{9,10,18\}$ & $\{13,17,2\}$ \\
\hline$\{2,5,11\}$ & $\{6,9,15\}$ & $\{20,0,4\}$ & $\{9,11,14\}$ & $\{10,11,19\}$ & $\{17,0,6\}$ \\
\hline$\{5,8,14\}$ & $\{3,6,12\}$ & & $\{4,6,9\}$ & $\{11,12,20\}$ & \\
\hline$\{8,11,17\}$ & $\{0,3,9\}$ & & $\{20,1,4\}$ & $\{12,13,0\}$ & \\
\hline
\end{tabular}

Table 19: Ucycles of rank two for two of the seven nonisomorphic cyclic STS(21)s 


\begin{tabular}{|c|c|c|}
\hline \multicolumn{3}{|c|}{ Base Blocks } \\
\hline $\multicolumn{3}{|c|}{0,1,9},\{0,2,5\},\{0,11,17\},\{0,7,14\}$ \\
\hline \multicolumn{3}{|c|}{ Ucycle } \\
\hline \multirow{2}{*}{\multicolumn{3}{|c|}{$\begin{array}{c}0,7,17,2,9,3,19,14,9,4,20,15,10,5,0, \\
16,11,6,1,17,12,7,4,11,3,4,5,6,7,8, \\
9,10,11,12,13,14,15,16,17,18,19,20, \\
0,1,2,18,13,8,3,14,4,15,5,19,8,18,3, \\
17,11,1,12,2,13,6,16,1,15,9,20,10 \\
\text { Blocks Represented by Ucycle } \\
\text { (read column-wise) } \\
\end{array}$}} \\
\hline & & \\
\hline$\{0,7,14\}$ & $\{3,4,12\}$ & $\{3,14,20\}$ \\
\hline$\{17,7,13\}$ & $\{4,5,13\}$ & $\{14,4,10\}$ \\
\hline$\{6,17,2\}$ & $\{5,6,14\}$ & $\{4,15,0\}$ \\
\hline$\{2,9,16\}$ & $\{6,7,15\}$ & $\{15,5,11\}$ \\
\hline$\{13,3,9\}$ & $\{7,8,16\}$ & $\{5,12,19\}$ \\
\hline$\{19,0,3\}$ & $\{8,9,17\}$ & $\{8,19,4\}$ \\
\hline$\{14,16,19\}$ & $\{9,10,18\}$ & $\{18,8,14\}$ \\
\hline$\{9,11,14\}$ & $\{10,11,19\}$ & $\{7,18,3\}$ \\
\hline$\{4,6,9\}$ & $\{11,12,20\}$ & $\{3,10,17\}$ \\
\hline$\{20,1,4\}$ & $\{12,13,0\}$ & $\{0,11,17\}$ \\
\hline$\{15,17,20\}$ & $\{13,14,1\}$ & $\{11,1,7\}$ \\
\hline$\{10,12,15\}$ & $\{14,15,2\}$ & $\{1,12,18\}$ \\
\hline$\{5,7,10\}$ & $\{15,16,3\}$ & $\{12,2,8\}$ \\
\hline$\{0,2,5\}$ & $\{16,17,4\}$ & $\{2,13,19\}$ \\
\hline$\{16,18,0\}$ & $\{17,18,5\}$ & $\{6,13,20\}$ \\
\hline$\{11,13,16\}$ & $\{18,19,6\}$ & $\{16,6,12\}$ \\
\hline$\{6,8,11\}$ & $\{19,20,7\}$ & $\{5,16,1\}$ \\
\hline$\{1,3,6\}$ & $\{20,0,8\}$ & $\{1,8,15\}$ \\
\hline$\{17,19,1\}$ & $\{0,1,9\}$ & $\{19,9,15\}$ \\
\hline$\{12,14,17\}$ & $\{1,2,10\}$ & $\{9,20,5\}$ \\
\hline$\{7,9,12\}$ & $\{18,20,2\}$ & $\{20,10,16\}$ \\
\hline$\{2,4,7\}$ & $\{13,15,18\}$ & $\{10,0,6\}$ \\
\hline$\{4,11,18\}$ & $\{8,10,13\}$ & \\
\hline$\{2,3,11\}$ & $\{3,5,8\}$ & \\
\hline
\end{tabular}

Table 20: A Ucycle of rank two for one of the seven nonisomorphic cyclic STS(21)s 


\begin{tabular}{|c|c|c|c|}
\hline \multicolumn{2}{|c|}{ Base Blocks } & \multicolumn{2}{|c|}{ Base Blocks } \\
\hline $\multicolumn{2}{|c|}{0,1,4},\{0,7,9\},\{0,5,10\}$ & $\multicolumn{2}{|c|}{0,1,4},\{0,2,9\},\{0,5,10\}$ \\
\hline & Ucycle & \multicolumn{2}{|r|}{ Ucycle } \\
\hline \multicolumn{2}{|c|}{$\begin{array}{l}0,7,6,9,4,1,2,3,4,5,0,14,2 \\
12,9,10,11,12,13,8,11,1,14 \\
6,13,5,12,4,11,3,10,2,9,1,8\end{array}$} & \multicolumn{2}{|c|}{$\begin{array}{c}0,2,1,4,9,6,7,8,13,12,11,10 \\
9,12,2,14,0,5,4,3,6,11,4,6 \\
8,10,12,14,1,3,5,7,9,11,13\end{array}$} \\
\hline \multicolumn{2}{|c|}{$\begin{array}{l}\text { Blocks Represented by Ucycle } \\
\text { (read column-wise) }\end{array}$} & \multicolumn{2}{|c|}{$\begin{array}{l}\text { Blocks Represented by Ucycle } \\
\text { (read column-wise) }\end{array}$} \\
\hline$\{0,7,9\}$ & $\{3,8,13\}$ & $\{0,2,9\}$ & $\{3,4,7\}$ \\
\hline$\{6,7,10\}$ & $\{7,8,11\}$ & $\{1,2,5\}$ & $\{2,3,6\}$ \\
\hline$\{5,6,9\}$ & $\{1,6,11\}$ & $\{0,1,4\}$ & $\{1,6,11\}$ \\
\hline$\{4,9,14\}$ & $\{7,14,1\}$ & $\{4,9,14\}$ & $\{2,4,11\}$ \\
\hline$\{0,1,4\}$ & $\{14,6,8\}$ & $\{5,6,9\}$ & $\{4,6,13\}$ \\
\hline$\{1,2,5\}$ & $\{6,13,0\}$ & $\{6,7,10\}$ & $\{6,8,0\}$ \\
\hline$\{2,3,6\}$ & $\{13,5,7\}$ & $\{7,8,11\}$ & $\{8,10,2\}$ \\
\hline$\{3,4,7\}$ & $\{5,12,14\}$ & $\{3,8,13\}$ & $\{10,12,4\}$ \\
\hline$\{4,5,8\}$ & $\{12,4,6\}$ & $\{12,13,1\}$ & $\{12,14,6\}$ \\
\hline$\{0,5,10\}$ & $\{4,11,13\}$ & $\{11,12,0\}$ & $\{14,1,8\}$ \\
\hline$\{14,0,3\}$ & $\{11,3,5\}$ & $\{10,11,14\}$ & $\{1,3,10\}$ \\
\hline$\{13,14,2\}$ & $\{3,10,12\}$ & $\{9,10,13\}$ & $\{3,5,12\}$ \\
\hline$\{2,7,12\}$ & $\{10,2,4\}$ & $\{8,9,12\}$ & $\{5,7,14\}$ \\
\hline$\{8,9,12\}$ & $\{2,9,11\}$ & $\{2,7,12\}$ & $\{7,9,1\}$ \\
\hline$\{9,10,13\}$ & $\{9,1,3\}$ & $\{13,14,2\}$ & $\{9,11,3\}$ \\
\hline$\{10,11,14\}$ & $\{1,8,10\}$ & $\{14,0,3\}$ & $\{11,13,5\}$ \\
\hline$\{11,12,0\}$ & $\{8,0,2\}$ & $\{0,5,10\}$ & $\{13,0,7\}$ \\
\hline$\{12,13,1\}$ & & $\{4,5,8\}$ & \\
\hline
\end{tabular}

Table 21: Ucycles of rank two for the two nonisomorphic cyclic STS(15)s 
Corollary 4.2.27. Every cyclic STS $(v), v \neq 3$, admits a Ucycle of rank two.

Proof. There are no cyclic STS $(v) \mathrm{s}$ for $v=4,5,6$. The trivial cyclic STS(3) does not admit a Ucycle of rank two as the minimum number of blocks that can be represented by a rank two Ucycle is two.

Corollary 4.2.28. Every cyclic TTS(v) admits a Ucycle of rank two.

Proof. There do not exist cyclic TTS $(v)$ s for $v=5,6$. The unique TTS(4) is generated by one base block: $\{0,1,2\}$. A Ucycle of rank two for this design is $U=0,1,2,3$. The two blocks of the trivial $\operatorname{TTS}(3)$ are represented by the Ucycle $U=0,1$.

Corollary 4.2.29. Every cyclic $(v, 4, \lambda)-B I B D$, with $v \geq 85$, admits a Ucycle of rank two.

Proof. Suppose $S$ is a cyclic $(v, 4, \lambda)$-BIBD. A $(v, 4, \lambda)$-BIBD may contain non-regular short orbit base blocks of the form $\{0, u, v / 2, u+v / 2\}, u \in \mathbb{Z}_{v} \backslash\{v / 4, v / 2,3 v / 4\}$. In addition, it may contain copies of the regular short orbit base block $\{0, v / 4, v / 2,3 v / 4\}$. The number of blocks in $S$ is $(v(v-1) \lambda) / 12$. Each full orbit base block generates $v$ of these blocks, each non-regular short orbit base block generates $v / 2$ of these blocks, and each regular short orbit base block generates $v / 4$ of these blocks, therefore,

$$
\frac{v(v-1) \lambda}{12}=v n_{1}+\frac{v}{2} n_{2}+\frac{v}{4} n_{3}
$$

which implies that the number of full orbit base blocks used to generate $S$ is

$$
n_{1}=\frac{(v-1) \lambda}{12}-\frac{n_{2}}{2}-\frac{n_{3}}{4}
$$


CHAPTER 4. GRAY CODES AND UNIVERSAL CYCLES FOR DESIGNS 201

As $n_{3} \leq \lambda, n_{1} \geq(v-4) \lambda / 12-n_{2} / 2$. We now divide our analysis into three cases.

Case 1: Suppose $n_{3}$ is even. Lemma 4.2 .24 implies that if $n_{1}+n_{2} \geq n_{3} / 2$, all regular short orbit base blocks can be inserted into collections of partial Ucycles. Since $n_{1} \geq(v-4) \lambda / 12-n_{2} / 2$ and $n_{3} \leq \lambda, n_{1}+n_{2} \geq n_{3} / 2$ holds for all quadruple systems with $(v-4) \lambda / 12+n_{2} / 2 \geq \lambda / 2$. As $(v-4) \lambda / 12+n_{2} / 2 \geq(v-4) \lambda / 12$, all regular short orbit base blocks can be inserted into collections of partial Ucycles when $(v-10) \lambda \geq 0$. This inequality holds for all $v \geq 10$.

We must also ensure there exists a full orbit base block with difference coprime to $v$ which can be used to create a length- $v$ partial Ucycle. When $\lambda=1$, there are no non-regular short orbit base blocks in $S$ (i.e. $n_{2}=0$ ). When $v \geq 10$, the element $1 \in \mathbb{Z}_{v}$ must appear as a difference in a full orbit base block, and so a length- $v$ partial Ucycle can be created. When $\lambda \geq 2$, we require $n_{2} \leq\lceil\lambda \phi(v) / 12\rceil-1$ to ensure the existence of a full orbit base block with difference coprime to $v$. Since $n_{2} \leq \lambda / 2$ and $\lambda \geq 2$, this inequality will hold for all $v$ with $\phi(v) \geq 12$. We conclude that for this case, every cyclic $(v, 4, \lambda)$-BIBD, $v \geq 31$, admits a Ucycle of rank two.

Case 2: Suppose $n_{3}$ is odd and $v / 4$ is even. To create a Ucycle for the blocks of $S$, we require the existence of two full orbit base blocks each containing a difference coprime to $v$; one block will be used in the application of Lemma 4.2 .19 and the other will be used to create a length- $v$ partial Ucycle to which all other partial Ucycles can be joined. The minimum number of non-regular base blocks containing at least one 
difference coprime to $v$ is $\lceil\lambda \phi(v) / 12\rceil$. When $\lambda=1, n_{2}=0$, so to ensure there are at least two full orbit base blocks each containing a difference coprime to $v$, we must have $\phi(v) / 12>1$. This inequality holds for all $v \geq 43$, however, since $v / 4$ must be even, we find that this inequality holds for all $(v, 4,1)$-BIBDs with $v \geq 32$. When $\lambda \geq 2$, we require $n_{2} \leq\lceil\lambda \phi(v) / 12\rceil-2$. Since $n_{2} \leq \lambda / 2$ and $\lambda \geq 2$, this inequality will hold for all $v$ with $\phi(v) \geq 18$. This inequality holds for all $v \geq 61$, however, since $v / 4$ must be even, we find that this inequality holds for all $(v, 4, \lambda)$-BIBDs with $v \geq 56$.

Insertion of regular short orbit blocks proceeds as follows. First insert a single copy of $\operatorname{Dev}(\{0, v / 4, v / 2,3 v / 4\})$ into a collection of partial Ucycles representing a full orbit base block containing a difference coprime to $v$ (using the method described in Lemma 4.2.19). Second, insert the remaining $n_{3}-1$ copies of $\operatorname{Dev}(\{0, v / 4, v / 2,3 v / 4\})$ into $\left(n_{3}-1\right) / 2$ other non-regular base blocks. This is done via $\left(n_{3}-1\right) / 2$ applications of Lemma 4.2.15. In total, insertions of regular short orbit blocks require $\left(n_{3}-1\right) / 2+1$ distinct non-regular base blocks. Since $n_{1}+n_{2} \geq(v-4) \lambda / 12$ and $n_{3} \leq \lambda$, we will have sufficient full orbit base blocks when $(v-4) \lambda / 12 \geq(\lambda-1) / 2+1$ holds. This inequality simplifies to $(v-10) \lambda \geq 6$, therefore, sufficient full orbit base blocks exist for all $v \geq 16$. We conclude that for this case, every cyclic $(v, 4, \lambda)$-BIBD, $v \geq 56$, admits a Ucycle of rank two.

Case 3: Suppose $n_{3}$ is odd and $v / 4$ is odd. To create a Ucycle for the blocks of $S$, we require the existence of four full orbit base blocks each containing a difference 
coprime to $v$. The four blocks ensure that the selection of two non-regular base blocks for use in the single block insertion (as described in Lemma 4.2.22) leaves us with at least two full orbit base blocks containing differences coprime to $v$. One of these blocks will be used in the application of Lemma 4.2 .19 and the other block will be used to create a length- $v$ partial Ucycle to which all other partial Ucycles can be joined. The minimum number of non-regular base blocks containing at least one difference coprime to $v$ is $\lceil\lambda \phi(v) / 12\rceil$. When $\lambda=1, n_{2}=0$, so we need at least four full orbit base blocks each containing a difference coprime to $v$, or three full orbit base blocks where one of these base blocks has all differences coprime to $v$. That is, we must have $\phi(v) / 12>2$. This inequality holds for all $v \geq 91$, however, since $v / 4$ must be odd, we find this inequality holds for all cyclic $(v, 4,1)$-BIBDs with $v \geq 85$. When $\lambda \geq 2$, we require $n_{2} \leq\lceil\lambda \phi(v) / 12\rceil-4$. Since $n_{2} \leq \lambda / 2$ and $\lambda \geq 2$, this inequality will hold for all $v$ with $\phi(v) \geq 30$. This inequality also holds for all $v \geq 91$, hence we find this inequality holds for all cyclic $(v, 4, \lambda)$-BIBDs, $\lambda \geq 2$, with $v \geq 85$.

Insertion of regular short orbit blocks proceeds as follows. First insert a single copy of $\operatorname{Dev}(\{0, v / 4, v / 2,3 v / 4\})$, less one block, into a collection of partial Ucycles representing a full orbit base block containing a difference coprime to $v$ (using the method described in Lemma 4.2.19). Second, insert the block leftover between two collections of partial Ucycles (using the method described in Lemma 4.2.22). Third, insert the remaining $n_{3}-1$ copies of $\operatorname{Dev}(\{0, v / 4, v / 2,3 v / 4\})$ into $\left(n_{3}-1\right) / 2$ other 
non-regular base blocks. This is done via $\left(n_{3}-1\right) / 2$ applications of Lemma 4.2.15. In total, insertions of short orbit blocks require $\left(n_{3}-1\right) / 2+3$ distinct non-regular base blocks. Since $n_{1}+n_{2} \geq(v-4) \lambda / 12$ and $n_{3} \leq \lambda$, we will have sufficient full orbit base blocks when $(v-4) \lambda / 12 \geq(\lambda-1) / 2+3$. This inequality simplifies to $(v-10) \lambda \geq 30$, therefore, sufficient full orbit base blocks exist for all $v \geq 40$. We conclude that every cyclic $(v, 4, \lambda)$-BIBD, $v \geq 85$, admits a Ucycle of rank two.

Corollary 4.2.30. Every cyclic $(v, 5, \lambda)$-BIBD, with $v \geq 76$, admits a Ucycle of rank two.

Proof. Suppose $S$ is a cyclic $(v, 5, \lambda)$-BIBD. As non-regular short orbit base blocks do not exist in $(v, 5, \lambda)$-BIBDs (i.e. $\left.n_{2}=0\right)$, all regular short orbit blocks in $S$ must be inserted into collections of partial Ucycles representing the blocks developed from full orbit base blocks. The number of blocks in $S$ is $(v(v-1) \lambda) / 20$. Each full orbit base block generates $v$ of these blocks, while each regular short orbit base block generates $v / 5$ of these blocks, therefore,

$$
\frac{v(v-1) \lambda}{20}=v n_{1}+\frac{v}{5} n_{3}
$$

which implies that the number of full orbit base blocks used to generate $S$ is

$$
n_{1}=\frac{(v-1) \lambda}{20}-\frac{n_{3}}{5}
$$

As $n_{3} \leq \lambda, n_{1} \geq(v-5) \lambda / 20$. We now divide our analysis into three cases. 
Case 1: Suppose $n_{3}$ is even. Lemma 4.2.24 implies that if $n_{1} \geq n_{3} / 2$, all regular short orbit base blocks can be inserted into collections of partial Ucycles representing full orbit base blocks. Since $n_{1} \geq(v-5) \lambda / 20$ and $n_{3} \leq \lambda, n_{1} \geq n_{3} / 2$ holds for all $(v, 5, \lambda)$-BIBDs with $(v-5) \lambda / 20 \geq \lambda / 2$. Simplifying this expression yields $(v-15) \lambda \geq 0$. Therefore, when $v \geq 15$, all regular short orbit blocks can be inserted into collections of partial Ucycles. Furthermore, when $v \geq 15$, the element $1 \in \mathbb{Z}_{v}$ must appear as a difference in a full orbit base block so there exists a length- $v$ partial Ucycle to which all other partial Ucycles can be joined. We conclude that for this case, every cyclic $(v, 5, \lambda), v \geq 15$, admits a rank two Ucycle.

Case 2: Suppose $n_{3}$ is odd and $v / 5$ is even. To create a Ucycle for the blocks of $S$, we require the existence of two full orbit base blocks each containing a difference coprime to $v$, or one full orbit base block with all of its differences coprime to $v$. In the first case, one block will be used in the application of Lemma 4.2.19 and the other will be used to create a length- $v$ partial Ucycle to which all other partial Ucycles can be joined. In the second case, note that one of the differences coprime to $v$ can be used to create a length- $v$ partial Ucycle and there will be another difference coprime to $v$ in the block that can be used in the application of Lemma 4.2.19. The minimum number of full orbit base blocks containing differences coprime to $v$ is $\lceil\lambda \phi(v) / 20\rceil$, so we require $\lambda \phi(v) / 20 \geq 1$ to ensure the existence of a length- $v$ partial Ucycle. This inequality holds for all $v \geq 61$. 
Insertion of regular short orbit blocks proceeds as follows. First insert a single copy of $\operatorname{Dev}(\{0, v / 5,2 v / 5,3 v / 5,4 v / 5\})$ into a collection of partial Ucycles representing a full orbit base block containing a difference coprime to $v$ (using the method described in Lemma 4.2.19). Second, insert the remaining $n_{3}-1$ copies of $\operatorname{Dev}(\{0, v / 5,2 v / 5,3 v / 5,4 v / 5\})$ into $\left(n_{3}-1\right) / 2$ other full orbit base blocks. This is done via $\left(n_{3}-1\right) / 2$ applications of Lemma 4.2.15. In total, insertions of short orbit blocks require $\left(n_{3}-1\right) / 2+1$ distinct full orbit base blocks. Since $n_{1} \geq(v-5) \lambda / 20$ and $n_{3} \leq \lambda$, we will have sufficient full orbit base blocks when $(v-5) \lambda / 20 \geq(\lambda-1) / 2+1$. This inequality simplifies to $(v-15 \lambda) \geq 10$, so sufficient full orbit base blocks exist for all $v \geq 25$. We conclude that for this case, every cyclic $(v, 5, \lambda), v \geq 61$, admits a rank two Ucycle.

Case 3: Suppose $n_{3}$ is odd and $v / 5$ is odd. To create a Ucycle for the blocks of $S$, we require the existence of four full orbit base blocks each containing a difference coprime to $v$, or three full orbit base blocks where one of these base blocks has all differences coprime to $v$. In the first case, the four blocks ensure that the selection of two blocks for use in the single block insertion (as described in Lemma 4.2.22) leaves us with at least two blocks, each containing at least one difference coprime to $v$. One of these blocks will be used in the application of Lemma 4.2.19 and the other block will be used to create a length- $v$ partial Ucycle to which all other partial Ucycles can be joined. In the second case, the full orbit base block with all differences coprime to 
CHAPTER 4. GRAY CODES AND UNIVERSAL CYCLES FOR DESIGNS 207

$v$ can be developed by a difference coprime to $v$ (yielding a length- $v$ partial Ucycle) while still fulfilling its other role (either in Lemma 4.2 .19 or 4.2 .22 ). The minimum number of full orbit base blocks containing differences coprime to $v$ is $\lceil\lambda \phi(v) / 20\rceil$, so we require $\lambda \phi(v) / 20>2$ (which actually leaves two full orbit base blocks all of whose differences must be coprime to $v$ ), to ensure existence of these blocks. This inequality holds for all $v \geq 151$, however, since $v / 5$ must be odd, we find this inequality holds for all cyclic $(v, 5, \lambda)$-BIBDs with $v \geq 76$.

Insertion of regular short orbit blocks proceeds as follows. First insert a single copy of $\operatorname{Dev}(\{0, v / 5,2 v / 5,3 v / 5,4 v / 5\})$, less one block, into a collection of partial Ucycles representing a full orbit base block containing a difference coprime to $v$ (using the method described in Lemma 4.2.19). Second, insert the block leftover between two collections of partial Ucycles (using the method described in Lemma 4.2.22). Third, insert the remaining $n_{3}-1$ copies of $\operatorname{Dev}(\{0, v / 5,2 v / 5,3 v / 5,4 v / 5\})$ (this can be done via $\left(n_{3}-1\right) / 2$ applications of Lemma 4.2 .15$)$ into $\left(n_{3}-1\right) / 2$ other full orbit base blocks. In total, insertions of short orbit blocks require $\left(n_{3}-1\right) / 2+3$ distinct full orbit base blocks. Since $n_{1} \geq(v-5) \lambda / 20$ and $n_{3} \leq \lambda$, we will have sufficient full orbit base blocks when $(v-5) \lambda / 20 \geq(\lambda-1) / 2+3$. This inequality simplifies to $(v-15) \lambda \geq 50$, so sufficient full orbit base blocks exist for all $v \geq 65$. We conclude that every cyclic $(v, 5, \lambda)$-BIBD, $v \geq 76$, admits a Ucycle of rank two. 
Corollary 4.2.31. (i) Every cyclic $(v, k, 1)-B I B D$, with $k \geq 3$ and $v$ prime, admits a Ucycle of rank two.

(ii) Every cyclic $(v, k, 1)-B I B D$, with $k \geq 3$ and $k \nmid v$, admits a Ucycle of rank two.

(iii) Every cyclic $(v, k, 1)-B I B D$, with $k \geq 3, k \mid v, v \geq(3 k-2) k$ and $\phi(v)>2 k(k-1)$, admits a Ucycle of rank two.

Proof. Let $S=(V, \mathcal{B})$ be a $(v, k, 1)$-BIBD, $k \geq 3$. Since $\lambda=1$, at most one regular short orbit base block may appear in $S$ and no non-regular short orbit base blocks appear in $S$. To see this, suppose there were a non-regular short orbit base block $B \in \mathcal{B}$ with $n=|\operatorname{Dev}(B)| . \quad B$ must contain at least one difference $d \in \mathbb{Z}_{v}$ where $d<n . \quad B$ must also contain the elements $\left\{n m(\bmod v): m \in \mathbb{Z}_{n}\right\}$ and $\{n m+d$ $\left.(\bmod v): m \in \mathbb{Z}_{n}\right\}$. The pair $\{0, n\}$ appears in $B$ and in $B+(n-d)$, which implies that $\lambda>1$. This is a contradiction to $\lambda=1$.

If $v$ is prime or if $k \nmid v$, no regular short orbit base block may appear in $S$. All differences coprime to $v$ appear in full orbit base blocks, therefore, there exists a length- $v$ partial Ucycle to which all other partial Ucycles can be joined. This proves statements $(i)$ and $(i i)$.

If $k \mid v, S$ may contain a regular short orbit base block. If $S$ does not contain a regular short orbit base block, all differences coprime to $v$ appear in full orbit base blocks, therefore, there exists a length- $v$ partial Ucycle to which all other partial Ucycles can be joined. Suppose $S$ contains a regular short orbit base block. The 
number of full orbit base blocks in $S$ is $(v-k) /(k(k-1))$, since these base blocks must generate $v(v-1) /(k(k-1))-v / k$ blocks. To create a Ucycle for the blocks of $S$, we require the existence of four full orbit base blocks each containing a difference coprime to $v$, or three full orbit base blocks where one of these base blocks has all differences coprime to $v$. In the first case, the four blocks ensure that the selection of two blocks for use in the single block insertion (Lemma 4.2.22) leaves us with at least two blocks containing differences coprime to $v$. One of these blocks will be used in the application of Lemma 4.2.19 and the other block will be used to create a length- $v$ partial Ucycle to which all other partial Ucycles can be joined. In the second case, the full orbit base block with all differences coprime to $v$ can be developed by a difference coprime to $v$ (yielding a length- $v$ partial Ucycle) while still fulfilling its other role (either in Lemma 4.2 .19 or 4.2 .22 ). The minimum number of full orbit base blocks containing differences coprime to $v$ is $\lceil\phi(v) /(k(k-1))\rceil$, so we require $\phi(v) /(k(k-1))>2$ (which actually leaves two full orbit base blocks all of whose differences must be coprime to $v$ ), to ensure existence of these blocks.

Insertion of the regular short orbit blocks proceeds as follows. First insert all blocks of $\operatorname{Dev}(\{0, v / k, 2 v / k, \ldots,(k-1) v / k\})$, less one block, into a collection of partial Ucycles representing a full orbit base block containing a difference coprime to $v$ (using the method described in Lemma 4.2.19). Second, insert the block leftover between two collections of partial Ucycles (using the method described in Lemma 4.2.22). 
In total, these insertions require three distinct full orbit base blocks, therefore, we require $(v-k) /(k(k-1)) \geq 3$. We conclude that there exist sufficient full orbit base blocks for the insertion of regular short orbit blocks when $v \geq(3 k-2) k$.

Note that for a given $k$, there exists $v_{0} \in \mathbb{Z}$ such that for all $v \geq v_{0}$, the inequalities required for Corollary 4.2.31 (iii) must hold.

\section{The Construction in Action}

The procedure for creating a Ucycle of rank two for the blocks of a cyclic $(v, k, \lambda)$ BIBD is easily expressed as an algorithm. Assume we have a cyclic $(v, k, \lambda)$-BIBD satisfying the requirements of Theorem 4.2.3.

- Determine if there will be a single regular short orbit block remaining to be inserted. This will occur if the number of regular short orbit base blocks in the design is odd and $v / k$ is odd. Find non-regular base blocks $B_{d}=\left\{0, d_{1}, \ldots, d_{(k-1)}\right\}$ and $B_{f}=\left\{0, f_{1}, \ldots, f_{(k-1)}\right\}$ such that $f_{t}-d_{s}$ is a multiple of $v / k$. Create collections of partial Ucycles representing the blocks of $\operatorname{Dev}\left(B_{d}\right)$ and $\operatorname{Dev}\left(B_{f}\right)$, with $d_{1} \neq d_{s}$ and $f_{1} \neq f_{t}$, respectively, used to form the sequences. Set these collections of partial Ucycles aside.

- If there are an odd number of regular short orbit base blocks in the design, find a full orbit base block, distinct from $B_{d}$ and $B_{f}$, that contains a difference coprime to $v / k$. Denote this base block $B_{p}$ and denote the difference $d_{p}$. Create 
a collection of partial Ucycles representing the blocks of $\operatorname{Dev}\left(B_{p}\right)$, not using $d_{p}$ for development, and set the collection aside.

- Create a collection of partial Ucycles representing the blocks generated by each of the remaining non-regular base blocks. There is at least one full orbit base block containing a difference coprime to $v$, denote this block $B_{q}$. Ensure that $\operatorname{Dev}\left(B_{q}\right)$ is represented by a length- $v$ partial Ucycle by developing the partial Ucycle representing these blocks by a difference coprime to $v$.

- Pair the regular short orbit base blocks and for each pair insert the two sets of blocks developed from the pair into a collection of partial Ucycles.

- If there is a regular short orbit base block remaining, insert all blocks generated by this base block (or all except one if the number of blocks generated is odd) into the collection of partial Ucycles representing $\operatorname{Dev}\left(B_{p}\right)$.

- If there is one remaining regular short orbit block, insert it between two of the partial Ucycles representing $\operatorname{Dev}\left(B_{d}\right)$ and $\operatorname{Dev}\left(B_{f}\right)$.

- Join all partial Ucycles to the partial Ucycle representing $\operatorname{Dev}\left(B_{q}\right)$ to form a Ucycle for the blocks of the design.

To further illustrate the construction, let us build a rank two Ucycle for the cyclic STS(27) generated by the full orbit base blocks $\{0,1,13\},\{0,2,7\},\{0,3,11\}$, 
$\{0,4,10\}$ and the regular short orbit base block $\{0,9,18\}$. In preparation for inserting the block that will be leftover after pairing the blocks in $\operatorname{Dev}(\{0,9,18\})$, choose $d_{2}=2$ from $\{0,2,7\}$ and $f_{2}=11$ from $\{0,3,11\}$ so that $f_{2}-d_{2}=9(\bmod 27)$. We will develop the base block $\{0,2,7\}$ by 7 and develop the base block $\{0,3,11\}$ by 3 to create collections of partial Ucycles representing the blocks of $\operatorname{Dev}(\{0,2,7\})$ and $\operatorname{Dev}(\{0,3,11\})$, respectively. Let us insert pairs of blocks developed from the regular short orbit base block into the partial Ucycle representing $\operatorname{Dev}(\{0,4,10\})$. If we develop $\{0,4,10\}$ by 4 , then, since $\operatorname{gcd}(10,27 / 3)=1$, we can pair and insert all blocks in $\operatorname{Dev}(\{0,9,18\})$, save one, into this collection of partial Ucycles. Finally, we choose to develop $\{0,1,13\}$ by 1 since this will create a length-27 partial Ucycle representing $\operatorname{Dev}(\{0,1,13\})$.

Figure 24 illustrates the insertions of pairs from $\operatorname{Dev}(\{0,9,18\})$ into the partial Ucycle representing $\operatorname{Dev}(\{0,4,10\})$. The relationship of endpoints of edges (when read in the clockwise direction) is indicated by colour. A red edge indicates decreasing by 4 - the difference used for development - while a blue edge represents increasing by 4 . The green edges indicate the inserted blocks and black edges indicate those edges to which directionality no longer applies because of a label change.

Figure 25 illustrates the insertion of the block $\{8,17,26\}$ which is the block of $\operatorname{Dev}(\{0,9,18\})$ remaining after pair insertion. The red edges indicate a partial Ucycle containing some of the blocks of $\operatorname{Dev}(\{0,3,11\})$. The blue edges indicate the partial 


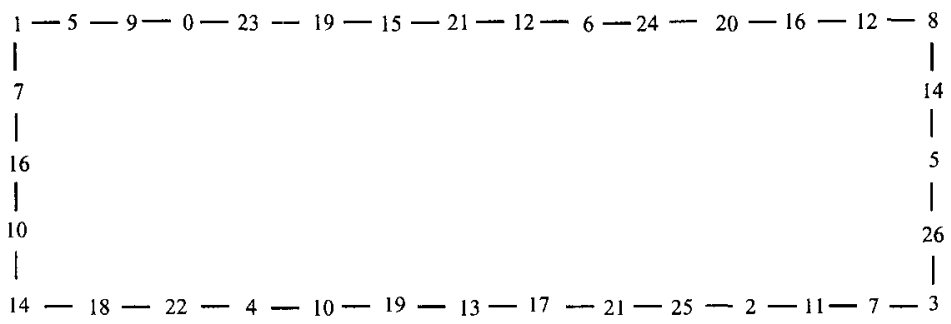

Figure 24: Insertion of pairs of blocks from $\operatorname{Dev}(\{0,9,18\})$ into the full-length partial Ucycle representing the blocks of $\operatorname{Dev}(\{0,4,10\})$

Ucycle representing the blocks of $\operatorname{Dev}(\{0,2,7\})$. Finally, the green edges are new edges and the gray point indicates the segment altered by the join.

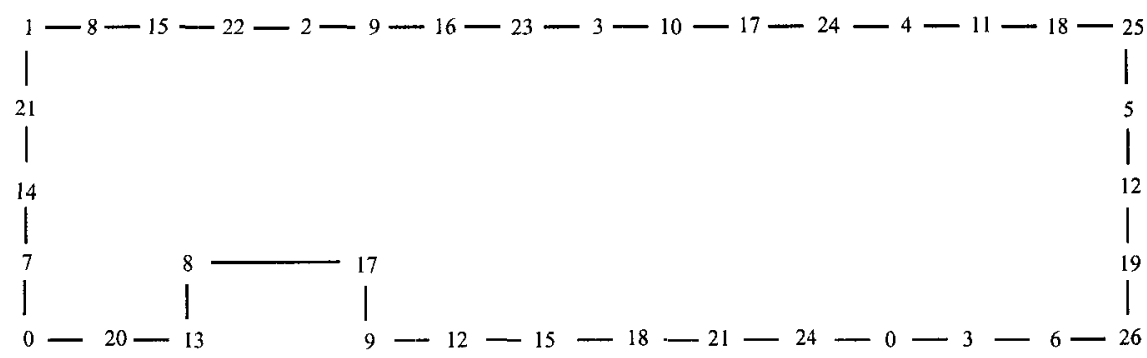

Figure 25: Insertion of the single remaining short block $\{8,17,26\}$

There remain several partial Ucycles untouched by the insertion of regular short orbit blocks. These partial Ucycles are shown in Figure 26.

All five partial Ucycles (which together contain all blocks of the design) can be joined together to create a Ucycle of rank two for the blocks of this $\operatorname{STS}(27)$. There are many ways to join these cycles together. Table 22 lists one of these ways. In this example we have joined all cycles to the partial Ucycle representing $\operatorname{Dev}(\{0,1,13\})$ (shown in Figure 26 and represented in Table 22 by bold entries). 


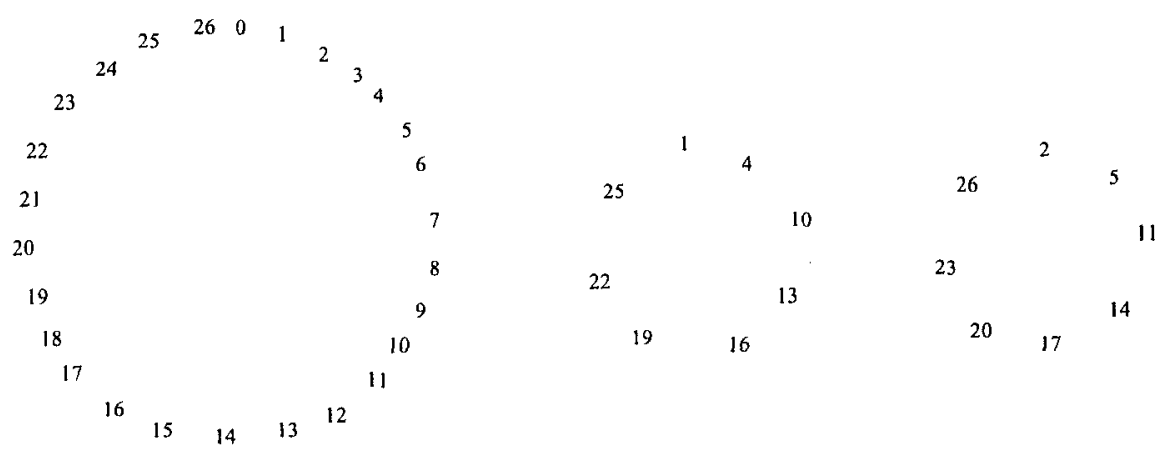

Figure 26: Other (unaltered) partial Ucycles representing the blocks of $\operatorname{Dev}(\{0,1,13\})$ and some of the blocks of $\operatorname{Dev}(\{0,3,11\})$

0141013161922251251114

$172023262 \mathbf{3 4 5 5 6 7 8} 1452637$

11225211713191042218141016

715902319152112624201612

89101112131470201362619

1252518114268036912151821

$24171032316922215812114 \mathbf{1 5}$

1617181920212223242526

Table 22: A Ucycle of rank two representing the blocks of the STS(27) generated by the base blocks $\{0,1,13\},\{0,2,7\},\{0,3,11\},\{0,4,10\}$ and $\{0,9,18\}$

The construction method presented in this section can be applied to cyclic $(v, K, \lambda)$ PBDs, with $\min K \geq 3$. All lemmas regarding the creation of collections of partial Ucycles and the insertion of regular short orbit blocks hold when $\min K \geq 3$. However, what relationship is required between the number of full, non-regular short and regular short orbit base blocks to ensure all regular short orbit blocks can be inserted into partial Ucycles and that all partial Ucycles can be joined to form a complete Ucycle for the design is yet to be determined. To ensure there exist sufficient non-regular 
base blocks for insertion of all regular short orbit blocks, we must take into account that a cyclic $(v, K, \lambda)$-PBD may contain several different sizes of regular short orbit base block. Note that if we have a cyclic $(v, K, \lambda)-\mathrm{PBD}$, with $2 \in K$, collections of partial Ucycles for base blocks of size two can be created as long as the base block is not a regular short orbit base block (A regular short orbit base block of size two has the form $\{0, v / 2\}$ and can only occur when $v \equiv 0(\bmod 2))$. A problem may occur because the partial Ucycles created from full orbit base blocks of size two cannot be used for insertion of a pair of distinct regular short orbit blocks of any size because there is only one way to represent these size-two blocks. That is, we do not have a $d_{s}$ in the full orbit base block that can be added to consecutive points in the partial Ucycle in order to insert two distinct short orbit blocks (see Lemma 4.2.13). In addition, we may encounter some difficulty in executing Lemma 4.2.22 if we discover that the only way to obtain a difference that is a multiple of $v / k$ from points in two different non-regular base blocks involves a base block of size two. To avoid specifying the exact conditions under which the required number of non-regular base blocks occur, we say that a cyclic $(v, K, \lambda)$-PBD for which all regular short orbit base blocks can be inserted into collections of partial Ucycles has a sufficient number of non-regular base blocks. Then we have the following generalization of Theorem 4.2.3.

Theorem 4.2.32. Let $S$ be a cyclic $(v, K, \lambda)-P B D$. If $\min K \geq 3$ and if $S$ has a sufficient number of non-regular base blocks (determined as described in the previous 
paragraph), then $S$ admits a Ucycle of rank two.

In Chapter 6, we discuss how to approach the determination of existence of Ucycles of rank two for general $(v, K, \lambda)$-PBDs. Recall that we have a non-existence result (Theorem 4.2.1) for an infinite family of PBDs having some blocks of size two; however, our existence result for cyclic $(v, k, \lambda)$-BIBDs leads us to believe that Ucycles of rank two exist for most $(v, K, \lambda)$-PBDs.

Recall that any ordering for the blocks of a design can be expressed in the terms of configuration ordering. Unfortunately, the expression of rank two Ucycles in the language of configuration orderings is not the most natural way to express these results. In order to specify that we must avoid the 3-claw, we equate rank two Ucycles with configuration orderings where each configuration has three blocks. A rank two Ucycle for a BIBD is equivalent to a $\mathcal{C}$-ordering for the blocks of the BIBD where $\mathcal{C}$ consists of all configurations having three blocks except the 3-claw. For triple systems, $\mathcal{C}$ consists of the configurations shown in Figure 27.

A similar situation occurs when translating Case and Pike's very strong result from [9]: the block intersection graph of every $(v, K, \lambda)$-PBD with $\max K \leq \lambda \min K$ is edge-pancyclic. However, as this result refers only to the relationship of pairs of consecutive blocks, we discuss it in terms of configurations involving two blocks. Consider the simple case of this result when $K=\{3\}$ and $\lambda \geq 2$. The block intersection graph of a $\operatorname{TS}(v, \lambda)$ has two vertices connected if and only if the blocks 

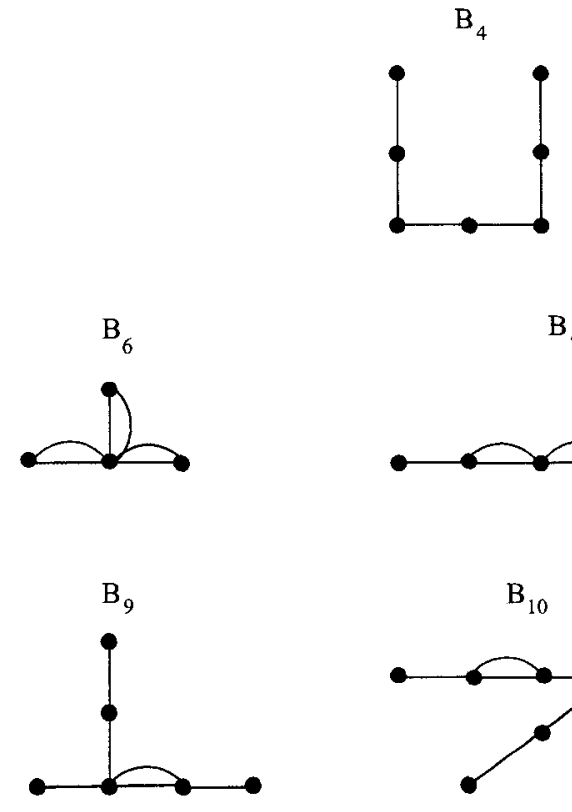
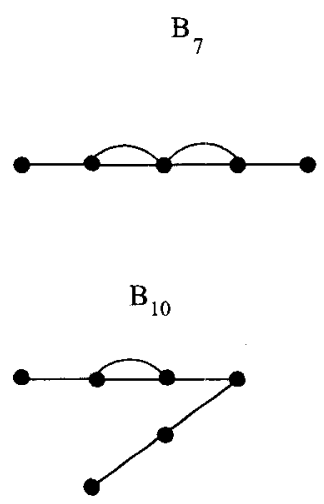
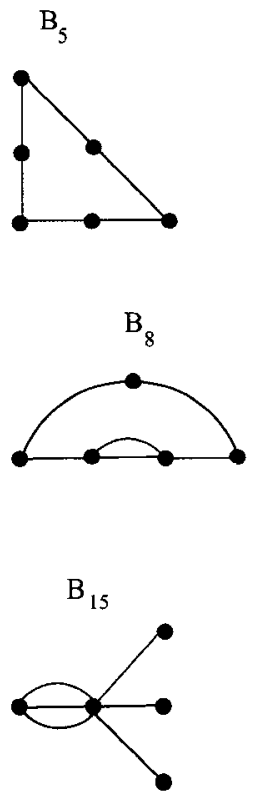

Figure 27: The set of configurations needed to translate the existence of Ucycles of rank two into the language of configuration ordering for triple systems

they represent intersect in one, two or three points. To translate such a result into a configuration ordering not only diminishes the result (because it does not require the edge-pancyclicity), but requires, even in this small case, that the set of configurations be all configurations on two blocks except the configuration of two disjoint blocks. That is, this result implies that every $\operatorname{TS}(v, \lambda)$ admits an $\left\{A_{2}, A_{3}, A_{4}\right\}$-cyclic ordering. In Chapter 5, we investigate the existence of related, but more natural, configuration orderings. 


\section{Chapter 5}

\section{New Results in Configuration}

\section{Ordering}

In this chapter, we look at configuration orderings for block designs. Recall that a configuration ordering is a listing of the blocks of a design such that every set of consecutive blocks of the necessary number is isomorphic to a given configuration. Cohen and Colbourn initiated the study of configuration ordering and they have been the main source of results in this area $[13,14,15,16,17]$. Their motivation for their investigations and some seminal results in the area were presented in Section 3.3. The published work in this area has dealt with orderings involving a single configuration and has focused on triple systems, with Steiner triple systems being the most 
frequently studied. In this chapter, we systematically address the existence of configuration orderings for triple systems where the configurations in question consist of two or three blocks. In doing so, we review known results in order to present new results in context. We begin by presenting a number of results that fill in gaps in our understanding of standard (single configuration) configuration ordering. In Section 5.2 , we turn to our new generalization of configuration ordering and establish the existence of several configuration orderings for triple systems in which each consecutive set of the specified number of blocks is isomorphic to a member of a set of allowable configurations. We conclude the chapter with configuration ordering results (both standard and generalized) for $(v, k, \lambda)$-BIBDs.

\subsection{Standard Configuration Ordering}

We begin with configurations involving two blocks. All such configurations are shown in Figure 1 (page 7). In [15] Cohen and Colbourn prove that, given a positive integer $\ell$, every $\operatorname{STS}(v)$ with $v \geq 81(\ell-1)+1$ admits a $D_{\ell}$-ordering, where $D_{\ell}$ denotes the configuration of $\ell$ disjoint triples. This result implies that every $\operatorname{STS}(v), v \geq 82$, admits an $A_{1}$-ordering. We show that every $\operatorname{TS}(v, \lambda), v \geq 17$, admits an $A_{1}$-cyclic ordering. Our proof is based on the fact that existence of an $A_{1}$-cyclic ordering is equivalent to existence of a Hamilton cycle in the complement of the block intersection graph of the design. 
Many of the proofs in this chapter will appeal to a well known theorem of Dirac.

Theorem 5.1.1. (See [58]) If $G=(V, E)$ has $|V| \geq 3$ and $\delta(G) \geq|V| / 2$ (where $\delta(G)$ denotes the minimum vertex degree of $G$ ), then $G$ is Hamiltonian.

Theorem 5.1.2. Every $T S(v, \lambda), v \geq 17$, admits an $A_{1}$-cyclic ordering.

Proof. Let $S=(V, \mathcal{B})$ be a $\operatorname{TS}(v, \lambda)$. The complement of the block-intersection graph of $S$ is the graph with vertex set $\mathcal{B}$ and edge set $\left\{\left(B_{i}, B_{j}\right): B_{i} \cap B_{j}=\emptyset, B_{i}, B_{j} \in \mathcal{B}\right\}$. Denote this graph $\overline{G_{S}}$. A Hamilton cycle in this graph yields an ordering of blocks such that every pair of consecutive blocks is disjoint. Under what conditions does this graph admit a Hamilton cycle? To apply Dirac's theorem, we must determine the minimum vertex degree in $\overline{G_{S}}$.

The degree of a vertex $w$ in $\overline{G_{S}}$ is governed by the number of blocks in $\mathcal{B}$ that intersect the block represented by $w$. Suppose $w$ represents the block $B$ and let $n_{1}$ denote the number of blocks containing exactly one point appearing in $B$, let $n_{2}$ denote the number of blocks containing exactly two points appearing in $B$, and let $n_{3}$ denote the number of blocks containing all three points appearing in $B$ (this count will include $B$ ). Since every point of the design appears in $r$ blocks, the points in $B$ appear in $3 r$ (non-unique) blocks. As the blocks containing two points that appear in $B$ will be counted twice by this calculation and the blocks containing three points that appear in $B$ will be counted three times by this calculation, we have $3 r=n_{1}+2 n_{2}+3 n_{3}$. On the other hand, there are three distinct pairs of points 
that can be made from the points appearing in $B$ and each of these pairs appears in $\lambda$ blocks of $S$. Since a block that is a copy of $B$ accounts for the appearance of three of these pairs, we have $3 \lambda=n_{2}+3 n_{3}$. The number of blocks intersecting $B$ is $n_{1}+n_{2}+n_{3}-1=3 r-3 \lambda+n_{3}-1$. Since the vertex $w$ representing $B$ is adjacent to the vertices representing blocks that do not intersect $B$, the minimum degree of $w$ is realized when the number of triples intersecting $B$ is maximized. The maximum value of $n_{3}$ is $\lambda$, therefore, the maximum number of vertices not adjacent to $w$ is $3 r-2 \lambda-1$. Hence, $\delta\left(\overline{G_{S}}\right) \geq(b-1)-(3 r-2 \lambda-1)$, where $b=v(v-1) \lambda / 6$.

In order to employ Theorem 5.1.1, we must determine when $\delta\left(\overline{G_{S}}\right) \geq b-3 r+2 \lambda \geq$ $b / 2$ holds. That is, we wish to determine bounds on $\lambda$ and $v$ such that the following inequality holds

$$
\frac{v(v-1) \lambda}{6}-\frac{3(v-1) \lambda}{2}+2 \lambda \geq \frac{v(v-1) \lambda}{12}
$$

Simplifying this inequality yields $v^{2}-19 v+42 \geq 0$, which holds for all $v \geq 17$ and arbitrary $\lambda$.

Recall that if $C$ is a configuration involving $\ell$ triples, then a $\operatorname{TS}(v, \lambda), S=(V, \mathcal{B})$, is said to be decomposable into copies of $C$ if either the set of triples $\mathcal{B}$, or the set of triples $\mathcal{B} \backslash T$, where $T$ is a subset of $\mathcal{B}$ containing at most $\ell-1$ triples, is decomposable into copies of $C$. We note that an immediate consequence of Theorem 5.1.2 is the following corollary, proved for STS $(v)$ s by Horák and Rosa in [33].

Corollary 5.1.3. Every $T S(v, \lambda), v \geq 17$, is decomposable into $A_{1}$ configurations. 
By proving that the 1-block intersection graph of every $\operatorname{TS}(v, \lambda)$, with $v \geq 12$ and arbitrary $\lambda$, is Hamiltonian [32], Horák, Pike and Raines proved that every $\operatorname{TS}(v, \lambda)$, $v \geq 12$, admits an $A_{2}$-cyclic ordering. The possible existence of $A_{3^{-}}$and $A_{4}$-orderings is restricted to triple systems with $\lambda>1$. The results we presented in Section 4.1 regarding the existence of 2-intersecting Gray codes imply that several infinite families of cyclic TTS $(v)$ s admit $A_{3}$-cyclic orderings. We restate Theorems 4.1 .4 and 4.1.6 in terms of configuration ordering.

Theorem 5.1.4. For each of the following orders there exists a TTS $(v)$ that admits an $A_{3}$-cyclic ordering:

(i) $v \equiv 1,4(\bmod 12)$, where $v \not \equiv 0(\bmod 5)$,

(ii) $v \equiv 3,7(\bmod 12), v \geq 7$.

The existence of $\operatorname{TTS}(v) \mathrm{s}$ with $v \equiv 0,6,9,10(\bmod 12)$ admitting $A_{3}$-orderings remains an open question. As $\mathrm{M}$. Colbourn and Johnstone have shown that there exist $\operatorname{TTS}(v)$ s that do not admit $A_{3}$-orderings [20], we feel the best general result we can hope to prove is the existence of a $\operatorname{TTS}(v)$ of each admissible order that admits an $A_{3}$-cyclic ordering for its blocks. Theorem 5.1.4 is half way to achieving this desired result. Finally, the only triple systems admitting $A_{4}$-orderings are those consisting of multiple (at least two) copies of a single block. These are the trivial designs: $\operatorname{TS}(3, \lambda)$, $\lambda \geq 2$. 
We turn now to configurations involving three blocks. All three block configurations in which each pair of blocks intersects in at most one point are shown in Figure 2 (page 8). Cohen and Colbourn's result regarding the existence of orderings where the configuration induced is a set of disjoint blocks implies that every $\operatorname{STS}(v), v \geq 163$, admits a $B_{1}$-ordering. We prove that every $\operatorname{TS}(v, \lambda), v \geq 103$, admits a $B_{1}$-cyclic ordering.

Theorem 5.1.5. Every $T S(v, \lambda), v \geq 103$, admits a $B_{1}$-cyclic ordering.

Proof. Let $S=(V, \mathcal{B})$ be a $\operatorname{TS}(v, \lambda)$. Although we can no longer use a block intersection graph to effectively represent the ordering question, we again cast the problem in graph theoretic terms. We begin by decomposing the blocks of $S$ into pairs of disjoint blocks. Corollary 5.1.3 states that such a decomposition exists provided that $v \geq 17$. Create a graph where each vertex represents one of the pairs of the decomposition and denote this graph $G$. If there is a block not paired in the decomposition, create a special vertex containing three disjoint triples. We must prove that such a vertex can be created.

Recall that the existence of an $A_{1}$-cyclic ordering for the blocks of a design is determined by the existence of a Hamilton cycle in the complement of the blockintersection graph for the design. To create the special vertex containing three disjoint triples, we find two consecutive blocks in this Hamilton cycle that are disjoint from the triple leftover after decomposition. Suppose the block $B \in \mathcal{B}$ is not paired in the 
decomposition of $S$ into $A_{1}$ configurations. Recall from the proof of Theorem 5.1.2 that the maximum number of blocks of $S$ that intersect $B$ (not including $B$ itself) in at least one point is $3 r-2 \lambda-1$. Suppose that no two consecutive blocks in the Hamilton cycle do not intersect $B$. What is the maximum number of blocks that can be in such a Hamilton cycle? To create pairs from the Hamilton cycle, we first remove the leftover block from the cycle, leaving a path where the first and last blocks do not intersect the leftover block. The worst possible arrangement in this path of the $3 r-2 \lambda-1$ blocks that intersect the leftover block is to have one appear every alternate block; such a sequence contains $2(3 r-2 \lambda-1)+1$ blocks. Therefore, if $6 r-4 \lambda-1<b-1$, we will be able to construct the special vertex. In terms of $v$ and $\lambda$, this requirement is $\left(v^{2}-19 v+42\right) \lambda>0$. Thus, for all $v \geq 17$ and arbitrary $\lambda$, if $|\mathcal{B}| \equiv 1(\bmod 2)$, the required special vertex containing three disjoint triples can be formed.

To complete the construction of $G$, join two vertices of $G$ by an edge if and only if the blocks represented by them are disjoint. We claim that a Hamilton cycle in $G$ will provide a $B_{1}$-cyclic ordering for the blocks of $S$. Figure 28 displays a portion of the graph $G$.

To see that the ordering of vertices given by a Hamilton cycle in $G$ is a $B_{1}$-cyclic ordering for the blocks they represent, consider the underlying blocks. Suppose the Hamilton cycle passes through $v_{0}, v_{6}, v_{4}, v_{10}$ (see Figure 28). This segment of the cycle 


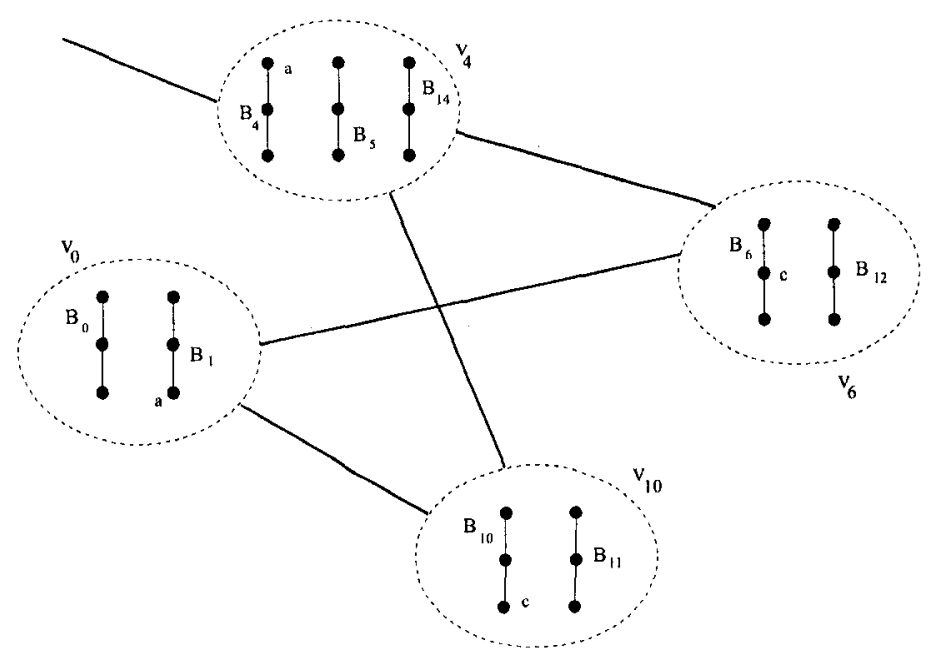

Figure 28: A portion of $G$ (for $B_{1}$-cyclic ordering)

represents the block ordering $B_{0}, B_{1}, B_{6}, B_{12}, B_{4}, B_{5}, B_{14}, B_{10}, B_{11}$. Every set of three consecutive blocks in this list forms a $B_{1}$ configuration. The order in which we list the blocks represented by a vertex is arbitrary.

To determine when $G$ admits a Hamilton cycle we appeal to Theorem 5.1.1. In order to determine the smallest possible minimum degree of a vertex in $G$, we begin by considering the blocks represented by a given vertex and determining the maximum number of blocks of $S$ that intersect these blocks. There are two cases to consider.

Case 1: $|\mathcal{B}| \equiv 0(\bmod 2)$. When the number of blocks in the design is even, no special vertex appears in $G$. Consider any vertex in $G$ and denote this vertex by $w$. The vertex contains two disjoint blocks and therefore six distinct points of $V$. Let $n_{1}$ denote the number of blocks containing exactly one of these six points, let $n_{2}$ denote the number of blocks containing exactly two of these six points, and 
let $n_{3}$ denote the number of blocks containing three of these points (this count will include the two blocks represented by $w$ ). Since every point of the design appears in $r$ blocks, the six points represented by $w$ appear in $6 r$ (non-unique) blocks. As blocks containing two of these points will be counted twice by this calculation and blocks containing three of these points will be counted three times by this calculation, we have $6 r=n_{1}+2 n_{2}+3 n_{3}$. On the other hand, there are fifteen distinct pairs of points that can be made from the six points represented by $w$ and each of these pairs appears in $\lambda$ blocks of the design. Since a block consisting of three of the six points accounts for the appearance of three of these pairs, we have $15 \lambda=n_{2}+3 n_{3}$. Finally, note that the two blocks represented by $w$ are included in $n_{3}$, so to determine the maximum number of blocks intersecting $w$, we maximize $n_{1}+n_{2}+n_{3}-2=6 r-15 \lambda+n_{3}-2$.

To determine the maximum value of $n_{3}$, note that the pairs of points that can be made from the points appearing in $w$ appear $15 \lambda$ times in the design. Each of the blocks containing three points that appear in $w$ accounts for three of these pairs, therefore $3 n_{3} \leq 15 \lambda$, which implies that $n_{3} \leq 5 \lambda$. We conclude that the maximum number of blocks that may intersect the blocks represented by $w$ is $6 r-15 \lambda+5 \lambda-$ $2=6 r-10 \lambda-2$. If each of these blocks appears as a member of a different pair of the decomposition (i.e. in a different vertex of $G$ ), then $w$ is not adjacent to any of these vertices. The number of vertices in $G$ is $v(v-1) \lambda / 12$, therefore, the minimum degree of a vertex in $G$ is $v(v-1) \lambda / 12-1-(6 r-10 \lambda-2)$. Hence, 
$\delta(G) \geq v(v-1) \lambda / 12-3(v-1) \lambda+10 \lambda+1$.

When is $\delta(G)$ greater than or equal to $|V(G)| / 2$ ? We must solve the following inequality

$$
\delta(G) \geq \frac{v(v-1) \lambda}{12}-3(v-1) \lambda+10 \lambda+1 \geq \frac{v(v-1) \lambda}{24} .
$$

Simplifying yields

$$
\begin{aligned}
v(v-1) \lambda-72(v-1) \lambda+240 \lambda+24 & \geq 0 \\
\lambda v^{2}-73 \lambda v+312 \lambda+24 & \geq 0 .
\end{aligned}
$$

All triple systems (with an even number of blocks) for which equation (5.1.3) holds satisfy the conditions of Dirac's theorem. That is, the graph $G$ induced by the triple system has a Hamilton cycle when $\lambda v^{2}-73 \lambda v+312 \lambda+24 \geq 0$. We conclude that every $\operatorname{TS}(v, \lambda)$, with $v \geq 69$ and an even number of blocks, admits a $B_{1}$-cyclic ordering.

Case 2: $|\mathcal{B}| \equiv 1(\bmod 2)$. When the number of blocks in $S$ is odd, a special vertex will appear in $G$. The special vertex has the largest potential number of blocks intersecting it due to the fact that it contains nine distinct points (versus six for all other vertices). Let $n_{1}$ denote the number of blocks containing exactly one of the points appearing in the special vertex, let $n_{2}$ denote the number of blocks containing exactly two of the points appearing in the special vertex, and let $n_{3}$ denote the number of blocks containing three points appearing in the special vertex (this 
count will include the three blocks represented by the special vertex). Using the same reasoning as in Case 1 , we have $9 r=n_{1}+2 n_{2}+3 n_{3}$ and $36 \lambda=n_{2}+3 n_{3}$. Note that the three blocks in the special vertex are included in $n_{3}$, so to determine the maximum number of blocks intersecting the blocks of the special vertex, we maximize $n_{1}+n_{2}+n_{3}-3=9 r-36 \lambda+n_{3}-3$.

To determine the maximum value of $n_{3}$, note that the pairs of points that can be made from the points appearing in the special vertex appear $36 \lambda$ times in the design. Each of the blocks containing three points that appear in the special vertex accounts for three of these pairs, therefore $3 n_{3} \leq 36 \lambda$, which implies that $n_{3} \leq 12 \lambda$. We conclude that the maximum number of blocks that may intersect the blocks of the special vertex is $9 r-36 \lambda+12 \lambda-3=9 r-24 \lambda-3$. If each of these blocks appears as a member of a different pair of the decomposition (i.e. in a different vertex of $G$ ), then the special vertex is not adjacent to any of these vertices. The number of vertices in $G$ is $(b-1) / 2=(v(v-1) \lambda-6) / 12$, therefore, the minimum degree of the special vertex is $(v(v-1) \lambda-6) / 12-1-(9 r-24 \lambda-3)$. Hence, $\delta(G) \geq(v(v-1) \lambda-6) / 12-9(v-1) \lambda / 2+24 \lambda+2$.

When is $\delta(G)$ greater than or equal to $|V(G)| / 2$ ? We must solve the following inequality

$$
\delta(G) \geq \frac{v(v-1) \lambda-6}{12}-\frac{9(v-1) \lambda}{2}+24 \lambda+2 \geq \frac{v(v-1) \lambda-6}{24} .
$$


Simplifying yields

$$
\begin{aligned}
v(v-1) \lambda-6-108(v-1) \lambda+576 \lambda+48 & \geq 0 \\
\lambda v^{2}-109 \lambda v+684 \lambda+42 & \geq 0 .
\end{aligned}
$$

All triple systems (with an odd number of blocks) for which equation (5.1.6) holds satisfy the conditions of Dirac's theorem. That is, the graph $G$ induced by the triple system has a Hamilton cycle when $\lambda v^{2}-109 \lambda v+684 \lambda+42 \geq 0$. We conclude that every $\operatorname{TS}(v, \lambda), v \geq 103$, admits a $B_{1}$-cyclic ordering.

Horák and Rosa have proved that every $\operatorname{STS}(v), v \geq 27$, can be decomposed into $B_{1}$ configurations [33]. Theorem 5.1.5 allows us to generalize this result.

Corollary 5.1.6. Every $T S(v, \lambda), v \geq 69$, can be decomposed into $B_{1}$ configurations. Proof. Let $S=(V, \mathcal{B})$ be a $\operatorname{TS}(v, \lambda)$. Case 1 of the proof of Theorem 5.1.5 deals with the existence of $B_{1}$-cyclic orderings when $b \equiv 0(\bmod 2)$, that is, when $G$ does not contain a special vertex. In this case, if $\lambda v^{2}-73 \lambda v+312 \lambda+24 \geq 0$, then $S$ admits a $B_{1}$-cyclic ordering. This inequality holds for all $v \geq 69$ and arbitrary $\lambda$. Such an ordering implies that the blocks of $S$ can be decomposed into $B_{1}$ configurations, with at most two blocks leftover. If $b \equiv 0(\bmod 6)$ this decomposition is exact.

When the number of blocks in $S$ is odd, there is a single block, say $B$, leftover after the decomposition of the design into $A_{1}$ configurations (the configurations that make up the vertices of $G$ ). Ignore this block, which can be arbitrarily chosen, and 
proceed to determine the existence of a Hamilton cycle in $G$. This construction allows us to avoid a special vertex, so the minimum degree of a vertex in $G$ is greater than or equal to $(b-1) / 2-1-(6 r-10 \lambda-2)=(v(v-1) \lambda-6) / 12-3(v-1) \lambda+10 \lambda+$ 1. Dirac's theorem (Theorem 5.1.1) implies that $G$ admits a Hamilton cycle when $(v(v-1) \lambda-6) / 12-3(v-1) \lambda+10 \lambda+1 \geq(v(v-1) \lambda-6) / 24$. Simplifying yields $\lambda v^{2}-73 \lambda v+312 \lambda+18 \geq 0$, which holds for all $v \geq 69$ and arbitrary $\lambda$. When $b \equiv 1,2$ $(\bmod 3)$, the Hamilton cycle can either be exactly partitioned into $B_{1}$ configurations, or can be partitioned into $B_{1}$ configurations with one block remaining, therefore, these triple systems are $B_{1}$-decomposable with either one or two blocks remaining. When $b \equiv 0(\bmod 3)$, the Hamilton cycle can be partitioned into $B_{1}$ configurations with two blocks remaining. In this case, there are three blocks of the triple system that are not members of a $B_{1}$ configuration, therefore, the triple system has not yet been decomposed into $B_{1}$ configurations. Note that the two blocks remaining after the Hamilton cycle has been decomposed into $B_{1}$ configurations must form an $A_{1}$ configuration. We must ensure that this $A_{1}$ configuration is disjoint from the leftover block $B$. Recall that the maximum number of blocks intersecting a given $A_{1}$ configuration is $6 r-10 \lambda-2$. Since $B$ can be arbitrarily chosen, to ensure we have an $A_{1}$ configuration that is disjoint from the leftover block, we simply require $b \geq(6 r-10 \lambda-2)+2+1$. Simplifying this inequality yields $\lambda v^{2}-19 \lambda v+78 \lambda-6 \geq$ 0 . This inequality holds for all $v \geq 17$, so the blocks of any $\operatorname{TS}(v, \lambda), v \geq 17$, 
can be decomposed into $A_{1}$ configurations such that there exists at least one $A_{1}$ configuration that is disjoint from the leftover block. To obtain a decomposition of $S$, determine a Hamilton cycle in the graph having $A_{1}$ configurations as vertices, then remove a vertex representing an $A_{1}$ configuration disjoint from the leftover block. Decompose the blocks in the Hamilton path into $B_{1}$ configurations (this is an exact decomposition) and form a $B_{1}$ configuration consisting of the disjoint $A_{1}$ configuration and the leftover block. We conclude that every $\operatorname{TS}(v, \lambda), v \geq 69$, can be decomposed into $B_{1}$ configurations.

Our survey of the literature on configuration ordering reveals that the smallest single configuration for which there are no known configuration ordering results is the $B_{2}$ configuration, otherwise known as the hut configuration. We employ the same proof technique as used in proving Theorem 5.1.5 to prove the existence of $B_{2}$-cyclic orderings.

Theorem 5.1.7. Every $T S(v, \lambda), v \geq 137$, is $B_{2}$-cyclic orderable.

Proof. Let $S=(V, \mathcal{B})$ be a $\operatorname{TS}(v, \lambda)$. We employ the following result of Horák, Pike and Raines: The 1-block intersection graph of any $\operatorname{TS}(v, \lambda)$, with $v \geq 12$ and arbitrary $\lambda$, is Hamiltonian [32]. We have previously noted that a direct result of this theorem is that every $\operatorname{TS}(v, \lambda), v \geq 12$, admits an $A_{2}$-cyclic ordering. More important to the current problem, the theorem implies that the blocks of any $\operatorname{TS}(v, \lambda), v \geq 12$, can be decomposed into pairs, with the possible exception of one block, such that the two 
blocks making up a pair share exactly one element. Suppose $B_{0}, B_{1}, \ldots, B_{b-2}, B_{b-1}$ is a Hamilton cycle in the 1-block intersection graph of $S$. We form pairs $\left\{B_{0}, B_{1}\right\}$, $\left\{B_{2}, B_{3}\right\}, \ldots$ and so on. This sequence of pairs ends in either $\left\{B_{b-2}, B_{b-1}\right\}$ or in $\left\{B_{b-3}, B_{b-2}\right\}$ with $B_{b-1}$ remaining.

Create a graph $G$ with a vertex representing each of the pairs determined by a Hamilton cycle in the 1-block intersection graph of $S$. If there is a block not paired, create a special vertex by amalgamating two vertices whose $A_{1}$ configurations intersect in a single point and whose blocks are disjoint from the leftover block. Include the leftover block in the amalgamated vertex (see Figure 29). We must prove that such an arrangement of blocks is possible.

To create the amalgamated vertex we find four consecutive blocks in the Hamilton cycle that are disjoint from the triple leftover after decomposition. Suppose the block $B \in \mathcal{B}$ is not paired. Recall from the proof of Theorem 5.1.2 that the maximum number of blocks intersecting $B$ is $3 r-2 \lambda-1$. Suppose we cannot find four consecutive blocks in the Hamilton cycle that do not intersect the leftover block. What is the maximum number of blocks that can be in such a Hamilton cycle? In decomposing the Hamilton cycle into $A_{2}$ configurations, we first remove the leftover block from the cycle, leaving a path where the first and last blocks intersect the leftover block in a single point. The worst possible arrangement in this path of the $3 r-2 \lambda-1$ blocks that intersect the leftover block is to have one appear every fourth block. Remembering 
that the path must start and end with intersecting blocks, we conclude that such a sequence contains $4(3 r-2 \lambda-2)+1$ blocks. Therefore, in order to ensure that we are able to construct the amalgamated vertex, we require $4(3 r-2 \lambda-2)+1<b-1$. In terms of $v$ and $\lambda$, this requirement is $\lambda v^{2}-37 \lambda v+84 \lambda+36>0$. Thus, for all $v \geq 35$ and arbitrary $\lambda$, an amalgamated vertex can be formed if $|\mathcal{B}| \equiv 1(\bmod 2)$. The amalgamated vertex has many possible forms; the three containing the largest number of vertices are given in Figure 29.

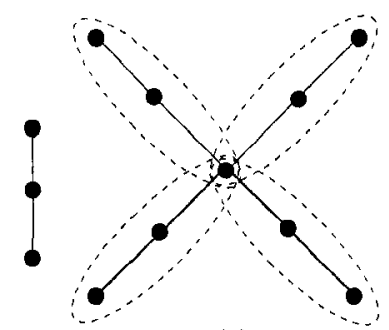

(a)

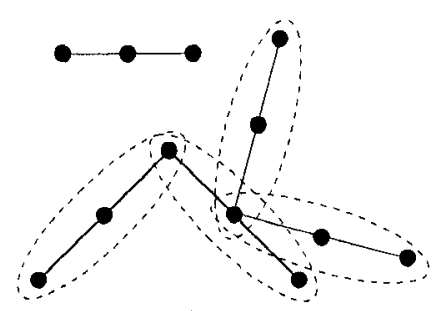

(b)

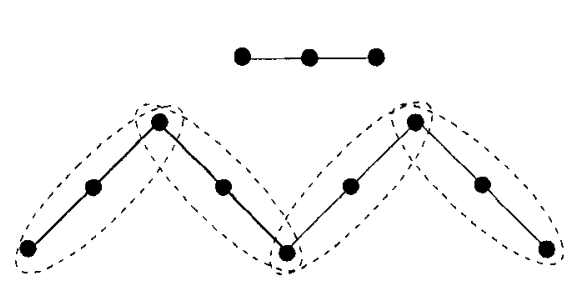

(c)

Figure 29: Forms of the amalgamated vertex containing the largest possible number of points

To complete the construction of $G$, join two vertices of $G$ by an edge if and only if the blocks represented by them are disjoint. We claim that a Hamilton cycle in $G$ will provide a $B_{2}$-cyclic ordering for the blocks of $S$. Figure 30 displays a portion of the graph $G$. 


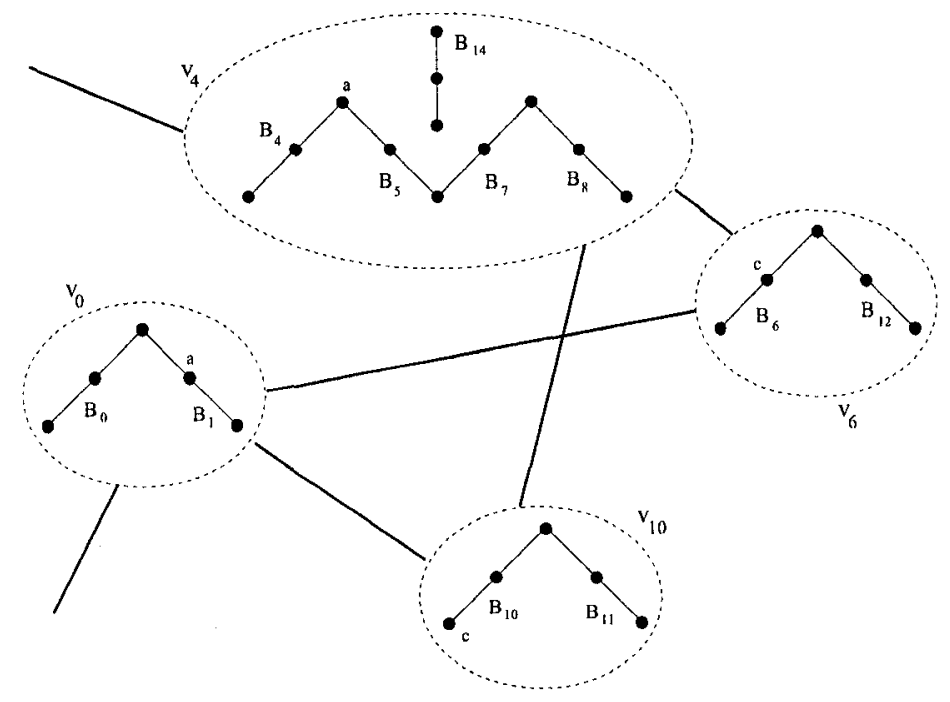

Figure 30: A portion of $G$ (for $B_{2}$-cyclic ordering)

To see that the ordering of vertices given by a Hamilton cycle in $G$ is a $B_{2}$-cyclic ordering for the blocks they represent, consider the underlying blocks. Suppose the Hamilton cycle passes through $v_{0}, v_{10}, v_{4}, v_{6}$ (see Figure 30 ). This segment of the cycle represents the block ordering $B_{0}, B_{1}, B_{10}, B_{11}, B_{4}, B_{5}, B_{14}, B_{7}, B_{8}, B_{6}, B_{12}$. The reader is invited to confirm that each set of three consecutive blocks in this list form a $B_{2}$ configuration. The order in which we list the blocks represented by a vertex is arbitrary, except when dealing with the amalgamated vertex. In general, the blocks of the amalgamated vertex will be listed as one of the $A_{2}$ configurations, followed by the disjoint block and then the remaining $A_{2}$ configuration. The amalgamated vertex labeled (a) in Figure 29 has four blocks that all intersect in the same point, therefore, any pairing of intersecting blocks may be selected.

To determine when $G$ admits a Hamilton cycle, we appeal to Theorem 5.1.1. In 
order to determine the smallest possible minimum degree of a vertex in $G$, we begin by considering the blocks represented by a given vertex and determining the maximum number of blocks of $S$ that intersect these blocks. There are two cases to consider.

Case 1: $|\mathcal{B}| \equiv 0(\bmod 2)$. When the number of blocks in the design is even, no amalgamated vertex appears in $G$. Consider any vertex in $G$ and denote this vertex by $w$. The vertex contains an $A_{2}$ configuration and therefore five distinct points of $V$. Let $n_{1}$ denote the number of blocks containing exactly one of these five points, let $n_{2}$ denote the number of blocks containing exactly two of these five points, and let $n_{3}$ denote the number of blocks containing three of these points (this count will include the two blocks represented by $w$ ). Since every point of the design appears in $r$ blocks, the five points represented by $w$ appear in $5 r$ (non-unique) blocks. As blocks containing more than one of these points are counted multiple times by this formula, we have $5 r=n_{1}+2 n_{2}+3 n_{3}$. On the other hand, there are ten distinct pairs of points that can be made from the five points appearing in $w$ and each of these pairs appears in $\lambda$ blocks of the design. Since a block containing three of the five points accounts for the appearance of three of these pairs, we have $10 \lambda=n_{2}+3 n_{3}$. Finally, note that the two blocks represented by $w$ are included in $n_{3}$, so to determine the maximum number of blocks intersecting $w$, we maximize $n_{1}+n_{2}+n_{3}-2=5 r-10 \lambda+n_{3}-2$.

To determine the maximum value of $n_{3}$, note that the pairs of points that can be made from the points appearing in $w$ appear $10 \lambda$ times in the design. Each 
of the blocks containing three points that appear in $w$ accounts for three of these pairs, therefore $3 n_{3} \leq 10 \lambda$, which implies that $n_{3} \leq(10 / 3) \lambda$. We conclude that the maximum number of blocks that may intersect the blocks represented by $w$ is $5 r-10 \lambda+(10 / 3) \lambda-2=5 r-(20 / 3) \lambda-2$. If each of these blocks appears as a member of a different pair of the decomposition (i.e. in a different vertex of $G$ ), then $w$ is not adjacent to any of these vertices. The number of vertices in $G$ is $v(v-1) \lambda / 12$, therefore, the minimum degree of a vertex in $G$ is $v(v-1) \lambda / 12-1-(5 r-(20 / 3) \lambda-2)$. Hence, $\delta(G) \geq v(v-1) \lambda / 12-(5 / 2)(v-1) \lambda+(20 / 3) \lambda+1$.

When is $\delta(G)$ greater than or equal to $|V(G)| / 2$ ? We must solve the following inequality

$$
\delta(G) \geq \frac{v(v-1) \lambda}{12}-\frac{5(v-1) \lambda}{2}+\frac{20}{3} \lambda+1 \geq \frac{v(v-1) \lambda}{24}
$$

Simplifying yields

$$
\begin{aligned}
v(v-1) \lambda-60(v-1) \lambda+160 \lambda+24 & \geq 0 \\
\lambda v^{2}-61 \lambda v+220 \lambda+24 & \geq 0 .
\end{aligned}
$$

All triple systems (with an even number of blocks) for which equation (5.1.9) holds satisfy the conditions of Dirac's theorem. That is, the graph $G$ induced by the triple system has a Hamilton cycle when $\lambda v^{2}-61 \lambda v+220 \lambda+24 \geq 0$. We conclude that every $\operatorname{TS}(v, \lambda)$, with $v \geq 58$ and an even number of blocks, admits a $B_{2}$-cyclic ordering. 
Case 2: $|\mathcal{B}| \equiv 1(\bmod 2)$. When the number of blocks in $S$ is odd, an amalgamated vertex will appear in $G$. The amalgamated vertex has the largest potential number of blocks intersecting it due to the fact that it contains at most twelve distinct points (versus five for all other vertices). Let $n_{1}$ denote the number of blocks containing exactly one of the points appearing in the amalgamated vertex, let $n_{2}$ denote the number of blocks containing exactly two of the points appearing in the amalgamated vertex, and let $n_{3}$ denote the number of blocks containing three points appearing in the amalgamated vertex (this count will include the five blocks represented by the amalgamated vertex). Using the same reasoning as in Case 1, we have $12 r=n_{1}+2 n_{2}+3 n_{3}$ and $66 \lambda=n_{2}+3 n_{3}$. Note that the five blocks in the amalgamated vertex are included in $n_{3}$, so to determine the maximum number of blocks intersecting the blocks of the amalgamated vertex, we maximize $n_{1}+n_{2}+n_{3}-5=12 r-66 \lambda+n_{3}-5$.

To determine the maximum value of $n_{3}$, note that the pairs of points that can be made from the points appearing in the amalgamated vertex appear $66 \lambda$ times in the design. Each of the blocks containing three points that appear in the amalgamated vertex accounts for three of these pairs, therefore $3 n_{3} \leq 66 \lambda$, which implies that $n_{3} \leq 22 \lambda$. We conclude that the maximum number of blocks that may intersect the blocks of the amalgamated vertex is $12 r-66 \lambda+22 \lambda-5=12 r-44 \lambda-5$. If each of these blocks appears as a member of a different pair of the decomposition (i.e. in a different vertex of $G$ ), then the amalgamated vertex is not adjacent to any of these 
vertices. The number of vertices in $G$ is $(b-1) / 2-1=(v(v-1) \lambda-18) / 12$, since the amalgamated vertex contains two $A_{2}$ configurations. Therefore, the minimum degree of the amalgamated vertex is $(v(v-1) \lambda-18) / 12-1-(12 r-44 \lambda-5)$. Hence, $\delta(G) \geq(v(v-1) \lambda-18) / 12-6(v-1) \lambda+44 \lambda+4$.

When is $\delta(G)$ greater than or equal to $|V(G)| / 2$ ? We must solve the following inequality

$$
\delta(G) \geq \frac{v(v-1) \lambda-18}{12}-6(v-1) \lambda+44 \lambda+4 \geq \frac{v(v-1) \lambda-18}{24} .
$$

Simplifying yields

$$
\begin{array}{r}
v(v-1) \lambda-144(v-1) \lambda+1056 \lambda+78 \geq 0 \\
\lambda v^{2}-145 \lambda v+1200 \lambda+78 \geq 0
\end{array}
$$

All triple systems (with an odd number of blocks) for which equation (5.1.12) holds satisfy the conditions of Dirac's theorem. That is, the graph $G$ induced by the triple system has a Hamilton cycle when $\lambda v^{2}-145 \lambda v+1200 \lambda+78 \geq 0$. We conclude that every $\operatorname{TS}(v, \lambda), v \geq 137$, admits a $B_{2}$-cyclic ordering.

Horák and Rosa [34] have independently proved the following corollary.

Corollary 5.1.8. Every $S T S(v), v \geq 136$, is $B_{2}$-cyclic orderable.

Let us now to turn to the question of $B_{2}$ decomposability of triple systems.

Corollary 5.1.9. Every $T S(v, \lambda), v \geq 58$, can be decomposed into $B_{2}$ configurations. 
Proof. Let $S=(V, \mathcal{B})$ be a $\operatorname{TS}(v, \lambda)$. Case 1 of the proof of Theorem 5.1.7 deals with the existence of $B_{2}$-cyclic orderings when $b \equiv 0(\bmod 2)$. That is, when $G$ does not contain an amalgamated vertex. In this case, if $\lambda v^{2}-61 \lambda v+220 \lambda+24 \geq 0$, then $S$ admits a $B_{2}$-cyclic ordering. This inequality holds for all $v \geq 58$ and arbitrary $\lambda$. Such an ordering implies that the blocks of $S$ can be decomposed into $B_{2}$ configurations, with at most two blocks leftover. If $b \equiv 0(\bmod 6)$ this decomposition is exact.

When the number of blocks in $S$ is odd, there is a single block, say $B$, leftover after the decomposition of the design into $A_{2}$ configurations (the configurations that make up the vertices of $G$ ). Ignore this block, which can be arbitrarily chosen, and proceed to determine the existence of a Hamilton cycle in $G$. This construction allows us to avoid an amalgamated vertex, so the minimum degree of a vertex in $G$ is greater than or equal to

$(b-1) / 2-1-(5 r-(20 / 3) \lambda-2)=(v(v-1) \lambda-6) / 12-(5 / 2)(v-1) \lambda+(20 / 3) \lambda+1$.

Dirac's theorem (Theorem 5.1.1) implies that $G$ admits a Hamilton cycle when $(v(v-$ 1) $\lambda-6) / 12-(5 / 2)(v-1) \lambda+(20 / 3) \lambda+1 \geq(v(v-1) \lambda-6) / 24$. Simplifying yields $\lambda v^{2}-61 \lambda v+220 \lambda+18 \geq 0$, which holds for all $v \geq 58$ and arbitrary $\lambda$. When $b \equiv 1,2$ (mod 3$)$, the Hamilton cycle can either be exactly partitioned into $B_{2}$ configurations, or can be partitioned into $B_{2}$ configurations with one block remaining, therefore, these triple systems are $B_{2}$-decomposable with either one or two blocks remaining. When $b \equiv 0(\bmod 3)$, the Hamilton cycle can be partitioned into $B_{2}$ configurations 
with two blocks remaining. In this case there are three blocks of the triple system that are not members of a $B_{2}$ configuration, therefore, the triple system has not yet been decomposed into $B_{2}$ configurations. Note that the two blocks remaining after the Hamilton cycle has been decomposed into $B_{2}$ configurations must form an $A_{2}$ configuration. We must ensure that this $A_{2}$ configuration is disjoint from the leftover block $B$. Recall that the maximum number of blocks intersecting a given $A_{2}$ configuration is $5 r-20 \lambda / 3-2$. Since $B$ can be arbitrarily chosen, to ensure we have an $A_{2}$ configuration that is disjoint from the leftover block, we simply require $b \geq(5 r-20 \lambda / 3-2)+2+1$. Simplifying this inequality yields $\lambda v^{2}-16 \lambda v+55 \lambda-6 \geq$ 0 . This inequality holds for all $v \geq 12$, so the blocks of any $\operatorname{TS}(v, \lambda), v \geq 12$, can be decomposed into $A_{2}$ configurations such that there exists at least one $A_{2}$ configuration that is disjoint from the leftover block. To obtain a decomposition of $S$, determine a Hamilton cycle in the graph having $A_{2}$ configurations as vertices, then remove a vertex representing an $A_{2}$ configuration disjoint from the leftover block. Decompose the blocks in the Hamilton path into $B_{2}$ configurations (this is an exact decomposition) and form a $B_{2}$ configuration consisting of the disjoint $A_{2}$ configuration and the leftover block. We conclude that every $\operatorname{TS}(v, \lambda), v \geq 58$, can be decomposed into $B_{2}$ configurations.

We can do slightly better for $\operatorname{STS}(v)$ s.

Corollary 5.1.10. Every $S T S(v), v \geq 57$, can be decomposed into $B_{2}$ configurations. 
This last result was proved by Horák and Rosa in [33], however, our approach is much less labour intensive.

Notice that the proofs of existence for $B_{1}$ - and $B_{2}$-cyclic orderings for triple systems are very similar in flavour to the proofs in Sections 2.5 and 2.6 which use the de Bruijn graph to prove existence of a de Bruijn sequence and the transition graph to prove existence of Ucycles for $k$-subsets of an $n$-set and for permutations.

We now turn to configurations of three blocks with no blocks disjoint. A $B_{3^{-}}$ ordering for the blocks of a triple system would require that one point appear in every block of the design. Indeed, consider four consecutive blocks in such an ordering and denote the blocks $W, X, Y$ and $Z$. As each set of three consecutive blocks must intersect in a single point we have $W \cap X \cap Y=\{x\}$. Since $X$ and $Y$ share the common point $x$ (and no others), $Z$ must also contain the point $x$ (and no other points in $X \cup Y$ ) since $X, Y$ and $Z$ also form a $B_{3}$ configuration. This property implies that $r=b$ and hence $v=3$. However, the blocks of a $\operatorname{TS}(3, \lambda)$ are copies of a single block, thus they cannot form a $B_{3}$ configuration, let alone a $B_{3}$-ordering.

The form of configurations $B_{4}$ and $B_{5}$ is more promising than $B_{3}$; however, we find they are more difficult to deal with than $B_{1}$ and $B_{2}$. This difficulty stems from the fact that we require detailed information about how the first and third blocks in each consecutive set of three triples are related. Such information is not communicated by the 1-block intersection graph of a triple system. We know of no results 
regarding the existence of $B_{4}$-orderings. A $B_{4}$-cyclic ordering for the blocks of a triple system yields a rank two Ucycle for the blocks of that triple system, however, as noted at the close of Chapter 4, a rank two Ucycle is not necessarily (and, in fact, is unlikely to be) a $B_{4}$-ordering. This relationship suggests that it may be possible to apply the methods developed in Chapter 4 for constructing Ucycles of rank two to the construction of $B_{4}$-cyclic orderings for cyclic $\operatorname{TS}(v, \lambda) \mathrm{s}$. In this case, we must construct cycles where consecutive blocks have exactly one point of intersection and where blocks at distance two (i.e. separated by a single block) are disjoint. These additional restrictions create difficulty immediately for our construction. In addition to the regular short orbit base block $\{0, v / 3,2 v / 3\}$, there are many other base blocks that do not admit a partial $B_{4}$-cyclic ordering for their developed blocks. Suppose $B_{d}$ is a base block of some cyclic $\operatorname{TS}(v, \lambda)$. A necessary condition for creating a partial $B_{4}$-cyclic ordering for $\operatorname{Dev}\left(B_{d}\right)$ is that the difference used for development appear exactly once in the base block. Therefore, a base block of the form $\{0, v / 4, v / 2\}$ does not admit a partial $B_{4}$-cyclic ordering. Other base blocks that are problematic include $\{0, v / 5,3 v / 5\},\{0, v / 5,2 v / 5\},\{0,2 v / 7,3 v / 7\},\{0, v / 6, v / 3\},\{0, v / 3, v / 2\}$, $\{0, v / 9, v / 3\}$ and $\{0, v / 4,3 v / 8\}$. Even if we restrict ourselves to cyclic STS $(v)$ s problems arise. In this case, the only base blocks whose developed blocks cannot be partially ordered are $\{0, v / 3,2 v / 3\},\{0,2 v / 7,3 v / 7\},\{0, v / 9, v / 3\}$ and $\{0, v / 4,3 v / 8\}$; however, the properties required of the base blocks whose partial $B_{4}$-cyclic orderings 
we wish to insert into are prohibitive. We will revisit the relationship of rank two Ucycles to configuration ordering in Section 5.2.

In [15], Cohen and Colbourn prove that for each order $v \geq 15$, there exists an $\operatorname{STS}(v)$ that does not admit a $B_{5}$-ordering. On the other hand, these authors conjecture that for each admissible order there exists an $\operatorname{STS}(v)$ that does admit a $B_{5^{-}}$ ordering. In the next section, we relax the definition of configuration ordering by allowing each consecutive set of blocks of the specified number to be isomorphic to one of several different configurations. This will allow us to obtain orderings involving $B_{4}$ and $B_{5}$ configurations.

\subsection{Generalized Configuration Ordering}

In light of the fact that we cannot determine the existence of $B_{4}$ - nor $B_{5}$-orderings by our graph theoretic method, and recalling our discussion in Section 4.2 on translation of rank two Ucycles into the language of configuration ordering, an interesting question to ask is: do there exist designs that admit orderings for their blocks where each consecutive set of blocks (of a specified size) is isomorphic to a configuration in a small (but larger than one) set of configurations? Recall the definition of a generalized configuration ordering: Let $\mathcal{C}$ be a set of configurations, each having $\ell$ blocks. A $\mathcal{C}$-ordering for a design is a listing of the blocks of the design such that every $\ell$ consecutive blocks form a configuration isomorphic to a configuration in $\mathcal{C}$. It is only 
interesting to look at generalized configuration orderings where the set consists of configurations for which standard configuration orderings are not known to exist. For example, since every $\operatorname{TS}(v, \lambda), v \geq 17$, admits an $A_{1}$-cyclic ordering (Theorem 5.1.2), if $\mathcal{C}$ is a set of configurations including $A_{1}$, then these same triple systems admit $\mathcal{C}$-cyclic orderings. Since any set containing $A_{2}$ is also uninteresting and we have partial results implying the existence of $A_{3}$-cyclic orderings, we will not look at sets of configurations where each configuration consists of two blocks. For sets of configurations where each configuration consists of three blocks, we ignore sets that contain $B_{1}$ or $B_{2}$. The existence of a Hamilton cycle in the 1-block intersection graph of every $\operatorname{TS}(v, \lambda), v \geq 12$, is equivalent to the existence of a $\left\{B_{3}, B_{4}, B_{5}\right\}$-cyclic ordering for each of these triple systems. What can be said about orderings where the set consists of two configurations from the set $\left\{B_{3}, B_{4}, B_{5}\right\}$ ? The most logical pair to consider is $\left\{B_{4}, B_{5}\right\}$, as an ordering involving these configurations allows pairs of consecutive blocks to intersect in exactly one point and excludes sets of three consecutive blocks intersecting in a single point. $\mathrm{A}\left\{B_{4}, B_{5}\right\}$-cyclic ordering for the blocks of a design is exactly a claw avoiding (or $B_{3}$-avoiding) Hamilton cycle in the 1-block intersection graph of the design. A design admitting such an ordering is said to be Eulerian.

Theorem 5.2.1. Every cyclic $S T S(v), v \neq 3$, admits a $\left\{B_{4}, B_{5}\right\}$-cyclic ordering. Proof. Let $S=(V, \mathcal{B})$ be an $\operatorname{STS}(v), v>3$. Corollary 4.2 .27 implies that $S$ admits a Ucycle of rank two. A Ucycle of rank two is equivalent to a $\mathcal{C}$-cyclic ordering, where $\mathcal{C}$ 
is the set of configurations given in Figure 27 (see page 217), however, since each pair of blocks in $\mathcal{B}$ intersect in at most one point, $\mathcal{C}$ is reduced to the set $\left\{B_{4}, B_{5}\right\}$.

The construction method used to create Ucycles of rank two can be adapted to create $\left\{B_{4}, B_{5}\right\}$-cyclic orderings for the blocks of cyclic triple systems in general. However, in addition to avoiding the creation of a claw configuration on three consecutive blocks, we must also avoid double intersections between pairs of consecutive blocks and double intersections of the first and third of three consecutive blocks. Construction proceeds in the following manner. Given a base block and the blocks developed from it, construct a $\left\{B_{4}, B_{5}\right\}$-cyclic ordering for these blocks. $A\left\{B_{4}, B_{5}\right\}$-cyclic ordering that does not contain all blocks of the given triple system is called a partial $\left\{\boldsymbol{B}_{4}, \boldsymbol{B}_{5}\right\}$-cyclic ordering. As in the construction of rank two Ucycles, there are some base blocks for which it is impossible to create a partial $\left\{B_{4}, B_{5}\right\}$-cyclic ordering for the blocks developed from it. We call all such blocks problem base blocks and formally define this concept in the next paragraph. Problem base blocks will be inserted into existing partial $\left\{B_{4}, B_{5}\right\}$-cyclic orderings (using insertion methods discussed in Section 4.2). Finally, all partial orderings are joined together to form a $\left\{B_{4}, B_{5}\right\}$-cyclic ordering for the triple system. Throughout the construction process it is necessary to avoid creating an ordering of blocks that induces configurations other than $B_{4}$ and $B_{5}$. The configurations that may be induced by a rank two Ucycle are given in Figure 27 (see page 217), thus, in constructing a $\left\{B_{4}, B_{5}\right\}$-cyclic ordering 
using this method we must avoid the configurations $\left\{B_{3}, B_{6}, B_{7}, B_{8}, B_{9}, B_{10}, B_{15}\right\}$.

Partial $\left\{B_{4}, B_{5}\right\}$-cyclic orderings for the blocks developed from a single base block can be created using the same method as that used to create partial Ucycles of rank two (see Lemmas 4.2.4 and 4.2.5). There are two types of base block that do not admit partial $\left\{B_{4}, B_{5}\right\}$-orderings for their developed blocks. Type 1 problem base blocks do not admit partial $\left\{B_{4}, B_{5}\right\}$-orderings because each difference appearing in the base block appears at least twice. If a difference in a type 1 problem base block were used to create an ordering (via the method described in Lemmas 4.2.4 and 4.2.5) the resulting ordering would have consecutive blocks intersecting in at least two points. Type 1 problem base blocks are $\{0, v / 3,2 v / 3\}$ and $\{0, v / 4,3 v / 4\}$. Type 2 problem base blocks do not admit partial $\left\{B_{4}, B_{5}\right\}$-orderings because developing by a difference that appears once in the base block will lead to a double intersection of the first and third blocks. Type 2 problem blocks are $\{0, v / 5,4 v / 5\}$ and $\{0,2 v / 5,3 v / 5\}$. The relationship of the differences in these two blocks means that each set of three consecutive blocks in an ordering created by developing by a difference that appears once $\left(3 v / 5\right.$ in the first and $v / 5$ in the second) form a $B_{8}$ configuration.

Since the blocks developed from a problem base block cannot be represented by a partial $\left\{B_{4}, B_{5}\right\}$-cyclic ordering, we must insert these blocks into existing partial $\left\{B_{4}, B_{5}\right\}$-cyclic orderings. What problems may be encountered when adding points to 
partial $\left\{B_{4}, B_{5}\right\}$-cyclic orderings or when joining partial $\left\{B_{4}, B_{5}\right\}$-cyclic orderings together? Blocks will always be inserted so that consecutive blocks in the new sequence intersect. It is necessary to avoid creating double intersections in pairs of consecutive blocks, to avoid creating the claw configuration on three consecutive blocks, and to avoid double intersections of the first and third of three consecutive blocks. Since we are constructing partial $\left\{B_{4}, B_{5}\right\}$-cyclic orderings using the same methods as were used to construct rank two Ucycles, we use a pair of points to represent each block in an ordering. This allows us to avoid claw creation altogether. It remains to address how to avoid creating the two types of double intersection.

We have completed much of the adaptation of the rank two Ucycle construction method to $\left\{B_{4}, B_{5}\right\}$-cyclic ordering. The new problem blocks require new arguments regarding their insertion; in particular, multiple insertions into the same collection of partial $\left\{B_{4}, B_{5}\right\}$-cyclic orderings must be done carefully. While we believe almost all cyclic triple systems admit $\left\{B_{4}, B_{5}\right\}$-cyclic orderings and that such orderings can be obtained via this method, we feel the method is inelegant and lengthy. We are currently investigating other avenues for proving the existence of $\left\{B_{4}, B_{5}\right\}$-cyclic orderings for triple systems. In closing this section, note that there do exist triple systems which are not $\left\{B_{4}, B_{5}\right\}$-cyclic orderable. For example, the TS $(5,3)$ generated by base blocks $\{0,1,2\}$ and $\{0,2,4\}$ is not $\left\{B_{4}, B_{5}\right\}$-orderable by our construction method or any other. 


\subsection{Configuration Ordering for BIBDs}

As defined in Section 2.2, a $(n, \ell)$-configuration is a collection of $n$ points on $\ell$ lines. All published work regarding configuration ordering, and almost all published work regarding the decomposition of designs into configurations, deals with triple systems. These two concepts are easily generalized to BIBDs and PBDs. In this section we look at configuration orderings for configurations having blocks of size $k, k \in \mathbb{Z}^{+}$. Given a configuration $C$ having blocks of size three, we will denote the configuration of the same form with blocks of size $k$ by $C^{\prime}$. In cases where it is not clear how the configuration should be generalized we will describe the configuration in detail. Not surprisingly, there are few known results in this area and all come from the translation of results in other areas into the language of configuration ordering. We discuss the possibility of generalizing the new results presented in the previous two sections and some of the obstacles to this generalization.

The existence of $A_{1}^{\prime}$-orderings (i.e. $(2 k, 2)$-orderings) in the restricted general case of $(v, k, 1)$-BIBDs is easy to determine using the method employed for triple systems (see the proof of Theorem 5.1.2).

Theorem 5.3.1. Every $(v, k, 1)$-BIBD, with $v \geq 2 k^{2}+1$, admits an $A_{1}^{\prime}$-cyclic ordering.

Proof. Let $S=(V, \mathcal{B})$ be a $(v, k, 1)$-BIBD. We follow the same argument as in the proof of Theorem 5.1.2, that is, we determine when the complement of the block 
intersection graph of $S$ admits a Hamilton cycle. Such a cycle is exactly an $A_{1}^{\prime}$-cyclic ordering since every pair of consecutive blocks is disjoint. We begin by determining the minimum degree of a vertex in the complement of the block intersection graph. Since $\lambda=1$, each block of $S$ intersects exactly $k(r-1)$ other blocks, where $r=(v-1) /(k-1)$. This implies that the minimum degree of a vertex in the complement of the block intersection graph is $(b-1)-k r+k$, where $b=v(v-1) / k(k-1)$. If the minimum vertex degree is equal to or exceeds half the number of vertices in the graph, the graph is Hamiltonian (Theorem 5.1.1). We must determine when $b-k r+k-1 \geq b / 2$. In terms of $v$ and $k$, this equation is equivalent to $v^{2}-\left(2 k^{2}+1\right) v+2 k\left(k^{2}-k+1\right) \geq 0$, which holds whenever

$$
v \geq \frac{2 k^{2}+1+\sqrt{4 k^{4}-8 k^{3}+12 k^{2}-8 k+1}}{2} .
$$

Note that $4 k^{4}-8 k^{3}+12 k^{2}-8 k+1$ (the quantity under the square root) is less than $4 k^{4}$ for all $k \geq 2$. Therefore, every $(v, k, 1)$-BIBD, with $v \geq\left(4 k^{2}+1\right) / 2$, admits an $A_{1}^{\prime}$-cyclic ordering.

Corollary 5.3.2. Every $(v, k, 1)-B I B D$ with $v \geq 2 k^{2}+1$ can be decomposed into $A_{1}^{\prime}$ configurations.

Determining the minimum degree of a vertex in the complement of the block intersection graph by counting the maximum number of blocks intersecting a given block is difficult for $\lambda>1$ and general $k$. A different approach is required to determine the existence of $A_{1}^{\prime}$-orderings for BIBDs in general. 
The reader may recall that in Section 3.3.2 we listed two results regarding generalized $A_{2}$-orderings. An $A_{2}^{\prime}$-ordering is a list in which pairs of consecutive blocks intersect in exactly one point. The first result is a restatement of the Hamiltonicity of the 1-block intersection graph of certain BIBDs [33]: every $(v, k, 1)$-BIBD admits a $(2 k-1,2)$-cyclic ordering. The second result is derived from the edge-pancyclicity of the 1-block intersection graph of certain PBDs [30]: every $(v, K, 1)$-PBD, with $\min (K) \geq 3$, admits a cyclic ordering in which each pair of consecutive blocks intersects in exactly one point. It would be interesting to investigate the existence of $A_{2}^{\prime}$-orderings for designs with $\lambda>1$.

The most natural generalization of the $A_{3}$ configuration to blocks of size $k$ is the $(k+1,2)$-configuration - a configuration on two blocks in which the blocks share all but two points. Of course, other possible generalizations are the configurations in which two blocks overlap in $m$ points, where $2 \leq m<k$, but, such configurations are not necessarily of interest and the existence of such orderings is highly dependent on the relationship of $m$ to $k$. Focusing on $(k+1,2)$-orderings, recall that both Ucycles of rank three and 2-intersecting Gray cycles for triple systems imply the existence of $(4,2)$-cyclic orderings for the blocks of triple systems. On the other hand, a $(k+1,2)$ cyclic ordering of the blocks of a $(v, k, \lambda)$-BIBD translates into a Ucycle of rank $k$ for the BIBD only in certain circumstances. For example, suppose a portion of a $(6,2)$ ordering for the blocks of a $(v, 5, \lambda)$-BIBD, with $\lambda \geq 4$, is $\{a, b, c, d, e\},\{b, c, d, e, f\}$, 
$\{b, c, d, e, g\}$. To express this list in Ucycle form, we begin with the point $a$, then some ordering of the remaining points in the first block, followed by the point $f$. However, $f$ is not in the third block and so a Ucycle of rank five cannot be formed from this configuration ordering. We know of no existence results regarding $(k+1,2)$-orderings for $(v, k, \lambda)$-BIBDs with $k>3$.

We turn to configurations having three blocks. Our method for proving the existence of $B_{1}$-cyclic orderings (see the proof of Theorem 5.1.5) can be employed to determine the existence of $B_{1}^{\prime}$-orderings (i.e. $(3 k, 3)$-orderings) for some $(v, k, \lambda)$ BIBDs. Our method requires the existence of an $A_{1}^{\prime}$ decomposition. We do not know of any published results regarding the decomposition of $(v, k, \lambda)$-BIBDs into $A_{1}^{\prime}$ configurations, but, Corollary 5.3.2 implies that all $(v, k, 1)$-BIBDs, with $v \geq 2 k^{2}+1$, admit decompositions into $A_{1}^{\prime}$ configurations.

Theorem 5.3.3. Every $(v, k, 1)$-BIBD, with $v \geq 12 k^{2}+1$, admits a $B_{1}^{\prime}$-cyclic ordering.

Proof. Let $S=(V, \mathcal{B})$ be a $(v, k, 1)$-BIBD with $v \geq 2 k^{2}+1$. Corollary 5.3.2 implies that the blocks of $S$ can be decomposed into $A_{1}^{\prime}$ configurations. Create a graph where each vertex represents one of the pairs of the decomposition and denote this graph $G$. If there is a block not paired in the decomposition, create a special vertex containing three disjoint triples. We must prove that such a vertex can be created.

Recall that the existence of an $A_{1}^{\prime}$-cyclic ordering for the blocks of a design is 
determined by the existence of a Hamilton cycle in the complement of the blockintersection graph for the design. To create the special vertex containing three disjoint blocks we find two consecutive blocks in this Hamilton cycle that are disjoint from the block leftover after decomposition. Suppose the block $B \in \mathcal{B}$ is not paired in the decomposition of $S$ into $A_{1}^{\prime}$ configurations. Recall that the number of blocks of $S$ that intersect $B$ in one point is $k(r-1)$ and that blocks cannot intersect in more than one point since $\lambda=1$. Suppose that no two consecutive blocks in the Hamilton cycle do not intersect $B$. What is the maximum number of blocks in such a Hamilton cycle? To create pairs from the Hamilton cycle, we first remove the leftover block from the cycle, leaving a path where the first and last blocks do not intersect the leftover block. The worst possible arrangement in this path of the $k(r-1)$ blocks that intersect the leftover block is to have one appear every alternate block; such a sequence contains $2 k(r-1)+1$ blocks. Therefore, if $2 k(r-1)<b-1$, we will be able to construct the special vertex. In terms of $v$ and $k$, this requirement is $v^{2}-\left(2 k^{2}+1\right) v+2 k^{3}-k^{2}+k>0$, which holds for all $v \geq 2 k^{2}+1$. Thus, if $|\mathcal{B}| \equiv 1$ (mod 2), the required special vertex containing three disjoint blocks can be formed.

To complete the construction of $G$, join two vertices of $G$ by an edge if and only if the blocks represented by them are disjoint. We claim that a Hamilton cycle in $G$ will provide a $B_{1}^{\prime}$-cyclic ordering for the blocks of $S$. To determine when $G$ admits a Hamilton cycle we appeal to Theorem 5.1.1. In order to determine the 
smallest possible minimum degree of a vertex in $G$, we begin by considering the blocks represented by a given vertex and determining the maximum number of blocks of $S$ that intersect these blocks. There are two cases to consider.

Case 1: $|\mathcal{B}| \equiv 0(\bmod 2)$. When the number of blocks in the design is even, no special vertex appears in $G$. Consider any vertex in $G$ and denote this vertex by $w$. The vertex contains two disjoint blocks, denote them $B_{1}$ and $B_{2}$, and therefore $2 k$ distinct points of $V$. Each of these points appears in $r-1$ other blocks, and each pair of points where one point is in $B_{1}$ and the other is in $B_{2}$ appear together in a unique block. Therefore, the number of blocks that intersect $v$ is $2 k(r-1)-k^{2}=$ $2 k(v-1) /(k-1)-2 k-k^{2}$. If each of these blocks appears as a member of a different pair of the decomposition (i.e. in a different vertex of $G$ ), then $w$ is not adjacent to any of these vertices. The number of vertices in $G$ is $v(v-1) /(2 k(k-1))$, therefore, the minimum degree of a vertex in $G$ is $v(v-1) /(2 k(k-1))-1-\left(2 k(v-1) /(k-1)-2 k-k^{2}\right)$. Hence, $\delta(G) \geq v(v-1) /(2 k(k-1))-2 k(v-1) /(k-1)+k^{2}+2 k-1$.

When is $\delta(G)$ greater than or equal to $|V(G)| / 2$ ? We must solve the following inequality

$$
\delta(G) \geq \frac{v(v-1)}{2 k(k-1)}-\frac{2 k(v-1)}{(k-1)}+k^{2}+2 k-1 \geq \frac{v(v-1)}{4 k(k-1)} .
$$

Simplifying yields

$$
\begin{array}{r}
v(v-1)-8 k^{2}(v-1)+4 k^{3}(k-1)+8 k^{2}(k-1)-4 k(k-1) \geq 0 \\
v^{2}-\left(8 k^{2}+1\right) v+4 k\left(k^{3}+k^{2}-k+1\right) \geq 0 .
\end{array}
$$


All $(v, k, 1)$-BIBDs (with an even number of blocks) for which equation (5.3.4) holds satisfy the conditions of Dirac's theorem. That is, the graph $G$ induced by the $(v, k, 1)$-BIBD has a Hamilton cycle when

$$
v \geq \frac{8 k^{2}+1+\sqrt{\left(8 k^{2}+1\right)^{2}-16 k\left(k^{3}+k^{2}-k+1\right)}}{2} .
$$

Since $\left(8 k^{2}+1\right)^{2}-16 k\left(k^{3}+k^{2}-k+1\right) \leq\left(8 k^{2}+1\right)^{2}$, we conclude that every $(v, k, 1)$ BIBD, with $v \geq 8 k^{2}+1$ and an even number of blocks, admits a $B_{1}^{\prime}$-cyclic ordering.

Case 2: $|\mathcal{B}| \equiv 1(\bmod 2)$. When the number of blocks in $S$ is odd, a special vertex will appear in $G$. The special vertex has the largest potential number of blocks intersecting it due to the fact that it contains $3 k$ distinct points (versus $2 k$ for all other vertices). Let $w$ denote the special vertex. Using the same reasoning as in Case 1, the maximum number of blocks intersecting the special vertex is $3 k(r-1)-3 k^{2}=$ $3 k(v-1) /(k-1)-3 k-3 k^{2}$. If each of these blocks appears as a member of a different pair of the decomposition (i.e. in a different vertex of $G$ ), then $w$ is not adjacent to any of these vertices. The number of vertices in $G$ is $(v(v-1) /(k(k-1))-1) / 2$, therefore, the minimum degree of a vertex in $G$ is $(v(v-1)-k(k-1)) /(2 k(k-1))-$ $1-\left(3 k(v-1) /(k-1)-3 k^{2}-3 k\right)$. Hence, $\delta(G) \geq(v(v-1)-k(k-1)) /(2 k(k-1))-$ $3 k(v-1) /(k-1)+3 k^{2}+3 k-1$.

When is $\delta(G)$ greater than or equal to $|V(G)| / 2$ ? We must solve the following 
inequality

$$
\begin{aligned}
\delta(G) & \geq \frac{v(v-1)-k(k-1)}{2 k(k-1)}-\frac{3 k(v-1)}{(k-1)}+3 k^{2}+3 k-1 \\
& \geq \frac{v(v-1)-k(k-1)}{4 k(k-1)} .
\end{aligned}
$$

Simplifying yields

$$
v^{2}-\left(12 k^{2}+1\right) v+12 k^{4}-5 k^{2}+5 k \geq 0
$$

All $(v, k, 1)$-BIBDs (with an odd number of blocks) for which equation (5.3.7) holds satisfy the conditions of Dirac's theorem. That is, the graph $G$ induced by the $(v, k, 1)$-BIBD has a Hamilton cycle when

$$
v \geq \frac{12 k^{2}+1+\sqrt{\left(12 k^{2}+1\right)^{2}-4\left(12 k^{4}-5 k^{2}+5 k\right)}}{2} .
$$

Since $\left(12 k^{2}+1\right)^{2}-4\left(12 k^{4}-5 k^{2}+5 k\right) \leq\left(12 k^{2}+1\right)^{2}$, we conclude that every $(v, k, 1)$ BIBD, with $v \geq 12 k^{2}+1$, admits a $B_{1}^{\prime}$-cyclic ordering.

Corollary 5.3.4. Every $(v, k, 1)-B I B D$, with $v \geq 12 k^{2}+1$, can be decomposed into $B_{1}^{\prime}$ configurations.

A $B_{2}^{\prime}$ configuration consists of two blocks intersecting in a single point, with a third block disjoint from both. Since every $(v, k, 1)$-BIBD admits an $A_{2}^{\prime}$-cyclic ordering, and hence a decomposition into $A_{2}^{\prime}$ configurations, we can employ the same method as used in the proof of Theorem 5.1.7 to determine the existence of $B_{2}^{\prime}$-orderings for $(v, k, 1)$-BIBDs. 
Theorem 5.3.5. Every $(v, k, 1)$-BIBD, with $v \geq 18 k^{2}-6 k+1$, admits a $B_{2}^{\prime}$-cyclic ordering.

Proof. Let $S=(V, \mathcal{B})$ be a $(v, k, 1)$-BIBD. $S$ can be decomposed into $A_{2}^{\prime}$ configurations, with the possible exception of one block, by finding a Hamilton cycle in the 1-block intersection graph of $S$. Create a graph $G$ where each vertex represents one of the pairs of the decomposition. If there is a block not paired, create a special vertex by amalgamating two vertices whose blocks intersect in a single point and whose blocks are disjoint from the leftover block. Include the leftover block in the amalgamated vertex. We must prove that such an arrangement of blocks is possible.

To create the amalgamated vertex we find four consecutive blocks in the Hamilton cycle that are disjoint from the block leftover after decomposition. Suppose the block $B \in \mathcal{B}$ is not paired and suppose we cannot find four consecutive blocks in the Hamilton cycle that do not intersect $B$. What is the maximum number of blocks that can be on such a Hamilton cycle? In decomposing the Hamilton cycle into $A_{2}$ configurations, we first remove the leftover block from the cycle, leaving a path where the first and last blocks intersect the leftover block in a single point. The worst possible arrangement in this path of the $k(r-1)$ blocks that intersect the leftover block is to have one appear every fourth block. Remembering that the path must start and end with intersecting blocks, we conclude that such a sequence contains $4(k r-k-1)+1$ blocks. Therefore, in order to ensure that we are able to construct 
the amalgamated vertex, we require $4(k r-k-1)+1<b-1$. In terms of $v$ and $k$, this requirement is $v^{2}-\left(4 k^{2}+1\right) v+4 k^{3}+2 k^{2}-2 k>0$. Thus, for all $v \geq 4 k^{2}+1$, an amalgamated vertex can be formed when $|\mathcal{B}| \equiv 1(\bmod 2)$.

To complete the construction of $G$, join two vertices of $G$ by an edge if and only if the blocks represented by them are disjoint. We claim that a Hamilton cycle in $G$ will provide a $B_{2}^{\prime}$-cyclic ordering for the blocks of $S$. To determine when $G$ admits a Hamilton cycle, we appeal to Theorem 5.1.1. The smallest possible minimum degree of a vertex in $G$ is calculated by considering the blocks represented by a given vertex and determining the maximum number of blocks of $S$ that intersect these blocks. There are two cases to consider.

Case 1: $|\mathcal{B}| \equiv 0(\bmod 2)$. When the number of blocks in the design is even, no amalgamated vertex appears in $G$. Consider any vertex in $G$ and denote this vertex by $w$. The vertex contains an $A_{2}^{\prime}$ configuration, denote the two member blocks $B_{1}$ and $B_{2}$, and therefore $2 k-1$ distinct points of $V$. Each of these points appears in $r-1$ other blocks, except for the point of intersection which appears in $r-2$ other blocks. Each pair of points where one point is in $B_{1}$ and the other is in $B_{2}$ (ignoring the point that appears in both blocks) appear together in a unique block. Therefore, the number of blocks that intersect $w$ is $(2 k-2)(r-1)+(r-2)-(k-1)^{2}=(2 k-1)(v-1) /(k-1)-$ $2 k-(k-1)^{2}$. If each of these blocks appears as a member of a different pair of the decomposition (i.e. in a different vertex of $G$ ), then $w$ is not adjacent to any of these 
vertices. The number of vertices in $G$ is $v(v-1) /(2 k(k-1))$, therefore, the minimum degree of a vertex in $G$ is $v(v-1) /(2 k(k-1))-1-(2 k-1)(v-1) /(k-1)+2 k+(k-1)^{2}$. Hence, $\delta(G) \geq v(v-1) /(2 k(k-1))-(2 k-1)(v-1) /(k-1)+(k-1)^{2}+2 k-1$.

When is $\delta(G)$ greater than or equal to $|V(G)| / 2$ ? We must solve the following inequality

$$
\delta(G) \geq \frac{v(v-1)}{2 k(k-1)}-\frac{(2 k-1)(v-1)}{(k-1)}+(k-1)^{2}+2 k-1 \geq \frac{v(v-1)}{4 k(k-1)}
$$

Simplifying yields

$$
v^{2}-\left(8 k^{2}-4 k+1\right) v+4 k\left(k^{3}-k^{2}+2 k-1\right) \geq 0
$$

All $(v, k, 1)$-BIBDs (with an even number of blocks) for which equation (5.3.9) holds satisfy the conditions of Dirac's theorem. That is, the graph $G$ induced by the $(v, k, 1)$-BIBD has a Hamilton cycle when

$$
v \geq \frac{8 k^{2}-4 k+1+\sqrt{\left(8 k^{2}-4 k+1\right)^{2}-16 k\left(k^{3}-k^{2}+2 k-1\right)}}{2} .
$$

Since $\left(8 k^{2}-4 k+1\right)^{2}-16 k\left(k^{3}-k^{2}+2 k-1\right) \leq\left(8 k^{2}-4 k+1\right)^{2}$, we conclude that every $(v, k, 1)$-BIBD, with $v \geq 8 k^{2}-4 k+1$ and an even number of blocks, admits a $B_{2}^{\prime}$-cyclic ordering.

Case 2: $|\mathcal{B}| \equiv 1(\bmod 2)$. When the number of blocks in $S$ is odd, an amalgamated vertex will appear in $G$. The amalgamated vertex has the largest potential number of blocks intersecting it due to the fact that it contains at most $5 k-3$ 
distinct points (versus $2 k-1$ for all other vertices). Consider the amalgamated vertex in Figure 29 (a) (with blocks of size $k$ ). Let $w$ denote the amalgamated vertex, let $B_{1}, B_{2}, B_{3}, B_{4}$ denote the four intersecting blocks and let $B_{5}$ denote the disjoint block. Each of the points in $w$ appears in $r-1$ other blocks, except for the point of intersection which appears in $r-4$ other blocks. Each pair of points where one point is in $B_{i}$ and the other is in $B_{j}, i \neq j, i, j \in\{1,2,3,4\}$ (ignoring the point of intersection) appear together in a unique block and each pair of points where one point is in $B_{i}$ and the other is in $B_{5}, i \in\{1,2,3,4\}$ appear together in a unique block. Finally, the point of intersection and each point in $B_{5}$ appear together in a unique block. Therefore, the number of blocks that intersect the amalgamated vertex is $(5 k-4)(r-1)+(r-4)-6(k-1)^{2}-4 k(k-1)-3$. Note that the same count is obtained when the other forms of amalgamated vertex are considered. If each of these blocks appears as a member of a different pair of the decomposition (i.e. in a different vertex of $G$ ), then the amalgamated vertex is not adjacent to any of these vertices. The number of vertices in $G$ is $(b-1) / 2-1=(v(v-1)-3 k(k-1)) /(2 k(k-1))$, since the amalgamated vertex contains two $A_{2}$ configurations. Therefore, the minimum degree of the amalgamated vertex is $(v(v-1)-3 k(k-1)) /(2 k(k-1))-1-(5 k-4)(r-1)-(r-4)+6(k-1)^{2}+4 k(k-1)+3$. Hence, $\delta(G) \geq(v(v-1)-3 k(k-1)) /(2 k(k-1))-(5 k-3)(v-1) /(k-1)+6(k-$ $1)^{2}+4 k(k-1)+5 k+2$. 
When is $\delta(G)$ greater than or equal to $|V(G)| / 2$ ? We must solve the following inequality

$$
\begin{aligned}
\delta(G) \geq & \frac{v(v-1)-3 k(k-1)}{2 k(k-1)}-(5 k-3) \frac{(v-1)}{(k-1)} \\
& +6(k-1)^{2}+4 k(k-1)+5 k+2 \\
\geq & \frac{v(v-1)-3 k(k-1)}{4 k(k-1)} .
\end{aligned}
$$

Simplifying yields

$$
\begin{array}{r}
v(v-1)-3 k(k-1)-4 k(5 k-3)(v-1)+24 k(k-1)^{3} \\
+16 k^{2}(k-1)^{2}+20 k^{2}(k-1)+8 k(k-1) \geq 0 \\
v^{2}-\left(20 k^{2}-12 k+1\right) v+40 k^{4}-84 k^{3}+93 k^{2}-41 k \geq 0 .
\end{array}
$$

All $(v, k, 1)$-BIBDs (with an odd number of blocks) for which equation (5.3.12) holds satisfy the conditions of Dirac's theorem. That is, the graph $G$ induced by the $(v, k, 1)$-BIBD has a Hamilton cycle when

$$
v \geq \frac{20 k^{2}-12 k+1+\sqrt{\left(20 k^{2}-12 k+1\right)^{2}-4\left(40 k^{4}-84 k^{3}+93 k^{2}-41 k\right)}}{2} .
$$

Since $\left(20 k^{2}-12 k+1\right)^{2}-4\left(40 k^{4}-84 k^{3}+93 k^{2}-41 k\right) \leq 240 k^{4}$ and $\sqrt{240}<16$ we conclude that every $(v, k, 1)$-BIBD, $v \geq\left(36 k^{2}-12 k+1\right) / 2$, admits a $B_{1}^{\prime}$-cyclic ordering.

Corollary 5.3.6. Every $(v, k, 1)-B I B D$, with $v \geq 18 k^{2}-6 k+1$, can be decomposed into $B_{2}^{\prime}$ configurations. 
A $B_{3}^{\prime}$ configuration is a configuration on three blocks with all blocks intersecting in a single point. Since such an ordering implies that every block in the design must contain a common point, the only $(v, k, \lambda)$-BIBDs that could admit a $B_{3}^{\prime}$-ordering must have $v=k$. However, such designs consist of copies of the same block and therefore cannot even be decomposed into $B_{3}^{\prime}$ configurations. Because we have no methods for dealing with $B_{4}$-orderings nor $B_{5}$-orderings for triple systems, we have no methods for embarking on an investigation into the existence of configuration orderings with generalized versions of these configurations. In [33], Horák and Rosa noted that obtaining a result regarding the decomposition of $(v, k, 1)$-BIBDs into triangles - as generalized $B_{5}$ configurations are known - is likely to be difficult. Of course, finding a triangle-ordering would imply the existence of such a decomposition, but such a result is likely to be even more difficult to obtain.

In Section 5.2 we introduced a generalization of configuration ordering in which each set of consecutive blocks of a specified number must form a configuration isomorphic to one of a set of allowable configurations. The only published result that can be reasonably translated into this language is Hare's pancyclicity of the 1-block intersection graph of certain PBDs: every $(v, K, 1)$-PBD, with $\min (K) \geq 3$, admits a $\left\{B_{3}^{\prime}, B_{4}^{\prime}, B_{5}^{\prime}\right\}$-cyclic ordering. Of course, this result is better expressed as an $A_{2}^{\prime}$-cyclic ordering since the original result is concerned only with the relationship of pairs of consecutive blocks, not triples of consecutive blocks. We have proved that all cyclic 
$\operatorname{STS}(v) \mathrm{s}, v>3$, admit $\left\{B_{4}, B_{5}\right\}$-cyclic orderings and we have a method to determine existence of $\left\{B_{4}, B_{5}\right\}$-cyclic orderings for cyclic $\operatorname{TS}(v, \lambda)$ s. However, this method (the adaptation of the construction method for Ucycles of rank two discussed in Section 5.2) becomes more difficult to use for general $k$ and $\lambda$, due to the multitude of possible forms for problem base blocks. In the special case of $\lambda=1$, we have the following result.

Lemma 5.3.7. Every cyclic $(v, k, 1)-B I B D$, with $k \nmid v$, is $\left\{B_{4}^{\prime}, B_{5}^{\prime}\right\}$-cyclic orderable.

Proof. Let $S$ be a $(v, k, 1)$-BIBD with $k \nmid v$. The only problem base blocks that may appear in a cyclic $(v, k, 1)$-BIBD are regular short orbit base blocks. Such base blocks have the form $\{0, v / k, 2 v / k, \ldots,(k-1) v / k\}$, therefore, since $k$ does not divide $v$ such base blocks do not appear in $S$. The design is generated entirely by full orbit base blocks. Applying the construction method described in Lemmas 4.2.4 and 4.2.5 to each of these base blocks yields a collection of partial $\left\{B_{4}^{\prime}, B_{5}^{\prime}\right\}$-cyclic orderings representing the blocks developed. Since every pair of points appears in exactly one block, no double intersection of blocks will occur. Furthermore, the difference $1 \in \mathbb{Z}_{v}$ appears in a full orbit base block and so there exists a length- $v$ partial $\left\{B_{4}^{\prime}, B_{5}^{\prime}\right\}$-cyclic ordering to which all other partial $\left\{B_{4}^{\prime}, B_{5}^{\prime}\right\}$-cyclic orderings can be joined (in the manner described in the proof of Theorem 4.2.3) to form a $\left\{B_{4}^{\prime}, B_{5}^{\prime}\right\}$-cyclic ordering for $S$. 
Another family of designs for which we understand the existence of $\left\{B_{4}^{\prime}, B_{5}^{\prime}\right\}$ orderings is symmetric designs.

Lemma 5.3.8. Every symmetric cyclic $(v, k, 1)-B I B D$ is $\left\{B_{4}^{\prime}, B_{5}^{\prime}\right\}$-cyclic orderable.

Proof. A symmetric cyclic design is generated by exactly one full orbit base block. Furthermore, when $\lambda=1$, each difference in $\mathbb{Z}_{v} \backslash\{0\}$ appears exactly once in the base block, therefore the base block is not a problem base block. The construction method described in the proof of Lemma 4.2 .4 can be applied using the difference one. The resulting length- $v$ partial $\left\{B_{4}^{\prime}, B_{5}^{\prime}\right\}$-cyclic ordering for the blocks developed from this base block is a $\left\{B_{4}^{\prime}, B_{5}^{\prime}\right\}$-cyclic ordering for the design.

Lemma 5.3.9. The blocks of a symmetric $(v, k, \lambda)-B I B D, \lambda>1$, cannot be $\left\{B_{4}^{\prime}, B_{5}^{\prime}\right\}$ ordered.

Proof. In a symmetric $(v, k, \lambda)$-BIBD (whether cyclic or non-cyclic) with $\lambda>1$, every pair of blocks intersects in at least two points, hence there is no ordering of the blocks that is a $\left\{B_{4}^{\prime}, B_{5}^{\prime}\right\}$-ordering.

There are many possible directions in which to take our research into the existence of standard and generalized configuration orderings. We discuss some of these in the final chapter. 


\section{Chapter 6}

\section{Conclusions and Future Work}

In this dissertation, we have looked at several rules for ordering the blocks of designs. Little work had been done in this area prior to the investigation laid out here. Cohen and Colbourn's work on configuration orderings for triple systems $[13,14,15,16]$ and the work of several groups of authors on the Hamiltonicity of the block intersection graph $[1,2,9,20,30,32,33]$ are the only previous work directly addressing ordering the blocks of designs. This dissertation began with the determination of natural and interesting ordering rules and a framework to describe and discuss orderings. We established three definitions for ordering the blocks of designs. Two of these definitions - Gray codes and Universal cycles -- are based on existing concepts of ordering other combinatorial objects, while the third - configuration orderings applies specifically to designs. We proved results regarding each of these types of 
ordering and discussed relationships between these concepts.

In Chapter 3 , we defined a $\kappa$-intersecting Gray code for a $(v, k, \lambda)$-BIBD to be a listing of the blocks of the design such that consecutive blocks intersect in exactly $\kappa$ points. We have shown that there exists a $\operatorname{TTS}(v)$, for each $v \equiv 1,3,4,7(\bmod 12)$, with sporadic exceptions, that admits a 2-intersecting Gray cycle for its blocks (Theorems 4.1.4 and 4.1.6). Furthermore, our work on rank two Ucycles implies that every cyclic $(v, k, 1)$-BIBD, having a sufficient number of full orbit base blocks, admits a 1-intersecting Gray cycle for its blocks (Theorem 4.2.3).

A stronger ordering rule defines a Ucycle for the blocks of a design. Chung, Diaconis and Graham introduced the concept of Ucycles for combinatorial objects in [12]. In order to discuss Ucycles for a particular combinatorial object, it is first necessary to determine a representation for the object. The size of the representation is called the rank of the Ucycle. We used two different definitions of rank for block designs: (1) block size, and (2) strength. When rank is equal to block size, each block is represented by itself. When rank is equal to strength, each block is represented by a subset of points that appear in the block, of size equal to strength. In particular, our work focused on Ucycles of rank three (referring to block size), and Ucycles of rank two (referring to strength). Natural questions to ask are: Do Ucycles of a given rank exist for all designs? If so, how do we construct them? We proved that there exists a $\operatorname{TTS}(v)$, for each $v \equiv 1,4,7(\bmod 12)$, with sporadic exceptions, admitting a . 
Ucycle of rank three (Theorem 4.1.4) and we have shown that Ucycles of rank three cannot exist for $\operatorname{TTS}(v) \mathrm{s}$, where $v \equiv 0,3,6,9(\bmod 12)$ (Lemma 3.2.3). Regarding the latter definition of rank, we proved that Ucycles of rank two exist for all cyclic $(v, k, \lambda)$-BIBDs, $k \geq 3$, having a sufficient number of full orbit base blocks (Theorem 4.2.3). This result was proved by construction, thereby answering the second question. Finally, we showed that there exists an infinite family of PBDs whose blocks do not admit Ucycles of rank two (Theorem 4.2.1).

In the process of constructing 2-intersecting Gray cycles and rank three Ucycles for TTS $(v) \mathrm{s}$, we defined a new concept which can be discussed and investigated independently of these ordering ideas. Let $D_{v}=d_{0}, d_{1}, \ldots, d_{\ell-1}$ be a sequence of elements from $\mathbb{Z}_{v} \backslash\{0\}$ such that $d_{i} \neq d_{j}(\bmod v)$ when $i \neq j$. Let $\sigma_{D_{v}}^{\prime}$ be the sequence of sums of pairs of consecutive elements in $D_{v}$, that is, $\sigma_{D_{v}}^{\prime}=d_{0}+d_{1}(\bmod v), d_{1}+d_{2}$ $(\bmod v), \ldots, d_{\ell-2}+d_{\ell-1}(\bmod v), d_{\ell-1}+d_{0}(\bmod v)$. If $v \equiv 0(\bmod 2)$, then $D_{v}$ is a cyclic difference sequence of order $v$ if and only if $d_{i} \neq v / 2$, for all $i \in\{0,1, \ldots, \ell-1\}$, and $2\left\{\left(\mathbb{Z}_{v} \backslash\{0\}\right) \backslash \pm\left\{D_{v}\right\}\right\}= \pm\left\{\sigma_{D_{v}}^{\prime}\right\}$. If $v \equiv 1(\bmod 2)$, then $D_{v}$ is a cyclic difference sequence of order $v$ if and only if $2\left\{\left(\mathbb{Z}_{v} \backslash\{0\}\right) \backslash \pm\left\{D_{v}\right\}\right\}= \pm\left\{\sigma_{D_{v}}^{\prime}\right\}$. Non-cyclic difference sequences are defined similarly (see Section 4.1 for details). The existence of a cyclic difference sequence of order $v$ implies the existence of a cyclic TTS $(v)$ and the existence of a non-cyclic difference sequence of order $v-1$ implies the existence 
of a 1-rotational TTS $(v)$ (Lemma 4.1.2). While we determined the existence of certain difference sequences with an eye to constructing 2-intersecting Gray cycles and rank three Ucycles for some cyclic triple systems, the question of whether difference sequences exist for all cyclic $\operatorname{TTS}(v)$ s is an interesting one. The definition of a difference sequence can be generalized to higher index; however, the placement of elements (either in the sequence or appearing as sums) becomes more complex.

Cohen and Colbourn defined the concept of configuration ordering and proved the existence of certain configuration orderings for Steiner triple systems [13]. In Chapter 5 , we determined the existence of several of these configuration orderings for $\operatorname{TS}(v, \lambda) \mathrm{s}$. In particular, we proved that every $\operatorname{TS}(v, \lambda), v \geq 17$, admits an $A_{1}$-cyclic ordering (Theorem 5.1.2) and every $\operatorname{TS}(v, \lambda), v \geq 103$, admits a $B_{1}$-cyclic ordering (Theorem 5.1.5). As configuration ordering is an emerging area of study, few configurations have been investigated. The smallest configuration that had not previously been considered was $A_{3}$. The existence of a 2-intersecting Gray code or a rank three Ucycle for a cyclic TTS $(v)$ of each order $v \equiv 1,3,4,7(\bmod 12)$, with sporadic exceptions, translates into the existence of $A_{3}$-cyclic orderings for these designs. The existence of one TTS $(v)$ of each admissible order that admits an $A_{3}$-cyclic ordering is an acceptable result, as the authors of [20] suggest that there exists an infinite family of TTS $(v)$ s (but only show the existence of one specific design) that do not admit such orderings. The next smallest configuration that had not been considered was $B_{2}$. We proved that every 
$\mathrm{TS}(v, \lambda), v \geq 137$, admits a $B_{2}$-cyclic ordering (Theorem 5.1.7). In many cases, the method used to prove existence of configuration orderings for triple systems can be extended to $(v, k, 1)$-BIBDs. We proved that every $(v, k, 1)$-BIBD, with $v \geq 2 k^{2}+1$, admits an $A_{1}^{\prime}$-cyclic ordering (Theorem 5.3.1). Furthermore, we proved that every $(v, k, 1)$-BIBD, with $v \geq 12 k^{2}+1$, admits a $B_{1}^{\prime}$-cyclic ordering (Theorem 5.3.3) and every $(v, k, 1)$-BIBD, with $v \geq 18 k^{2}-6 k+1$, admits a $B_{2}^{\prime}$-cyclic ordering (Theorem $5.3 .5)$

We introduced the concept of generalized configuration ordering for two reasons. The first is that it allows us to frame questions about existence and to discuss all orderings of blocks using a common language. The second is that it allows us to deal with configurations for which it is difficult to determine the existence of standard configuration orderings. Formally, let $\mathcal{C}$ be a set of configurations, each having $\ell$ blocks. A $\mathcal{C}$-ordering of a design is a listing of the blocks of the design such that each set of $\ell$ consecutive blocks forms a configuration isomorphic to a configuration in $\mathcal{C}$. Our work on rank two Ucycles allowed us to prove that every cyclic $\operatorname{STS}(v), v>3$, admits a $\left\{B_{4}, B_{5}\right\}$-cyclic ordering (Theorem 5.2.1) and every cyclic $(v, k, 1)$-BIBD, where $k \nmid v$, admits a $\left\{B_{4}^{\prime}, B_{5}^{\prime}\right\}$-cyclic ordering (Lemma 5.3.7). Furthermore, every symmetric cyclic $(v, k, 1)$-BIBD is $\left\{B_{4}^{\prime}, B_{5}^{\prime}\right\}$-cyclic orderable (Lemma 5.3.8).

Finally, we have corrected and completed two published results. The first relates to the existence of Ucycles for $k$-permutations of $[n]$. Jackson proved that for every 
integer $k \geq 3$ and every integer $n \geq k+1$, there exists a Ucycle for the $k$-permutations of $[n]([39])$, but he omitted several key details from his proof. We presented a full proof in Section 2.6.1. The second relates to the existence of $t$-cover Ucycles for $k$ subsets of $[n]$. Recall that $U(n, k)$ denotes the smallest $t$, given $n$ and $k$, such that a $t$-cover Ucycle exists for all $k$-subsets of an $n$-set. We proved that if $k$ is a prime power and $k$ divides $n$, then $U(n, k)=k$ (Corollary 2.6.12). A similar result appeared in [38], however, we found the proof of that result to be false. In Section 6.1, we will fill a gap in the proof of the Hamiltonicity of the block intersection graph of certain $(v, K, 1)$-PBDs which appears in [2].

While we have introduced new definitions and established new results in ordering the blocks of designs, there are many avenues for future research. One of the most natural is to expand the study of rank two Ucycles to general $(v, k, \lambda)$-BIBDs. The Ucycle concept is intended to be a framework under which all combinatorial objects can be ordered, therefore, we wish to show that the choice of rank based on strength is a reasonable one. We discuss the work we have done so far in pursuit of this result, and indicate the direction for future investigation in Section 6.1. In Section 6.2 we gather the conjectures stated throughout this dissertation and pose some additional questions. 


\subsection{Approaches to Determining the Existence of Rank Two Universal Cycles for all BIBDs}

We have been able to prove the existence of rank two Ucycles for most cyclic BIBDs. The methods involved in proving this result are heavily dependent on the structure of the automorphism; however, we would like to be able to determine the existence or non-existence of rank two Ucycles for all $(v, k, \lambda)$-BIBDs. In this section, we present the beginnings of our research in this direction. In particular, we establish a terminology and machinery to assist in tackling the problem. We begin by looking at the result of Alspach, Heinrich and Mohar on the Hamiltonicity of 1-block intersection graphs of PBDs [2], as we hope to use a generalization of their technique in solving our problem. Recall that for $I \subseteq \mathbb{N}$, the $I$-block intersection graph of a $(v, K, \lambda)$-PBD, $S=(V, \mathcal{B})$, is the graph whose vertex set is the blocks of the PBD and whose edge set is $E=\left\{\left(B_{1}, B_{2}\right):\left|B_{1} \cap B_{2}\right| \in I\right\}$. The block intersection graph of a $(v, K, \lambda)$-PBD has edge set $E=\left\{\left(B_{1}, B_{2}\right): B_{1} \cap B_{2} \neq \emptyset\right\}$. As mentioned in Section 3.3.2, many people have considered the question of whether the 1-block intersection graph of a design is Hamiltonian.

Theorem 6.1.1. ([2]) Let $S=(V, \mathcal{B})$ be a non-trivial pairwise balanced design with $\lambda=1, M$ the maximum size of a block in $\mathcal{B}$ and $m$ the minimum size of a block in B. If $M \leq 2 m$, then the block intersection graph of $S$ has a Hamilton cycle. 
Before presenting the proof of this theorem we introduce some additional terminology. Let $S=(V, \mathcal{B})$ be a block design. Recall that the block coloured pair adjacency graph of $S$ is the multigraph with vertex set $V$ such that, for each pair of distinct vertices $v_{1}$ and $v_{2}$, there is an edge between $v_{1}$ and $v_{2}$ of colour $B_{i}$ if $\left\{v_{1}, v_{2}\right\} \subseteq B_{i} \in \mathcal{B}$. Denote this graph $G^{V}$. Note that when $\lambda=1, G^{V}$ is the complete graph on $|V|$ vertices. We define a trail to be a walk in a multigraph in which no edge appears twice. A trail in $G^{V}$ is called block-dominating if the set of vertices in the trail intersect every block of $\mathcal{B}$. For example, the trail $0,1,3$ is block-dominating for the Fano plane (Figure 3 on page 18). Figure 31 illustrates $G^{V}$ for the Fano plane.

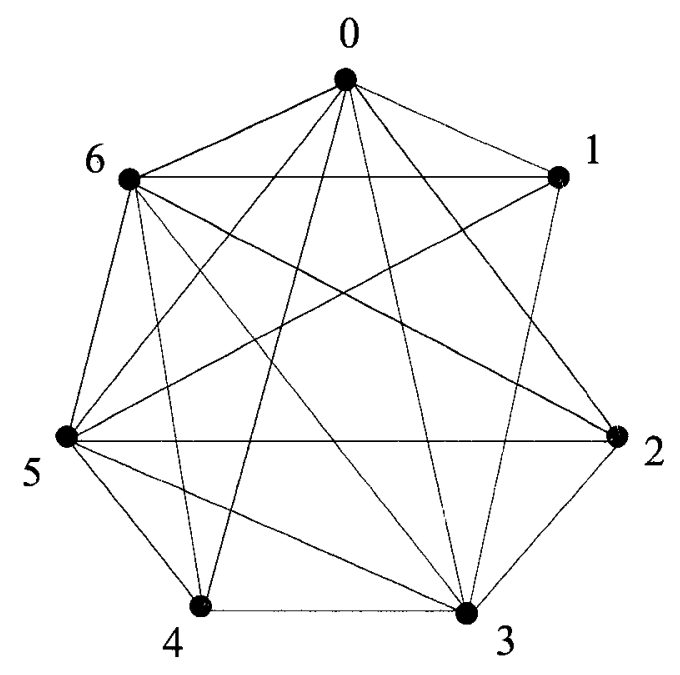

Figure 31: $G^{V}$ for the Fano plane

The following lemma will play a significant role in the proof of Theorem 6.1.1.

Lemma 6.1.2. ([2]) Let $S$ be a block design. The block intersection graph of $S$ is Hamiltonian if and only if $G^{V}$ has a block-dominating closed trail $W$ with no two 
edges of $W$ having the same colour.

Note that in Figure 31 the trail 0, 1, 3 is block-dominating but has all edges the same colour. The trail $0,1,6,3,2$ is a block-dominating closed trail with no two edges having the same colour.

Proof. Let $G_{S}$ denote the block intersection graph of $S=(V, \mathcal{B})$.

$(\Longrightarrow)$ Suppose $G_{S}$ has a Hamilton cycle, say $B_{0}, B_{1}, \ldots, B_{b-1}$. Let $a_{i} \in V$ be an element of $B_{i} \cap B_{i+1}$, where addition is performed modulo $b$. Consider the sequence $a_{0}, a_{1}, \ldots, a_{b-1}$ induced by the Hamilton cycle. For each contiguous subsequence $a_{i}, a_{i+1}, \ldots, a_{i+j}$ with $a_{i}=a_{i+1}=\ldots=a_{i+j}$, determine the maximal length of this subsequence and replace it by a single representative element. The closed trail obtained after applying this operation until it cannot be performed again is blockdominating (as the sequence contains an element from each block) and each of its edges has a different colour (simply use the rule of connecting elements $a_{i}$ and $a_{i+1}$ with edge colour $B_{i}$ ).

$\left(\Longleftarrow\right.$ Suppose $G^{V}$ has a block-dominating closed trail $W=a_{0}, e_{0}, a_{1}, e_{1}, \ldots, a_{\ell}$, $e_{\ell}, a_{0}$, with no two edges having the same colour. Let $B_{i}$ be the block "containing" the edge $e_{i}$, that is, let $\left\{a_{i}, a_{i+1}\right\} \in B_{i}$. We construct a Hamilton cycle in $G_{S}$ as follows. Let $C_{1}, C_{2}, \ldots, C_{t} \in \mathcal{B}$ be all the blocks containing $a_{0}$ whose colour does not appear in $W$. Then $P=C_{1}, C_{2}, \ldots, C_{t}, B_{0}$ is a trail in $G_{S}$. Let $C_{t+1}, C_{t+2}, \ldots, C_{t+r} \in \mathcal{B}$ be all the blocks containing $a_{1}$ which do not appear in the path $P$ and whose colour 
does not appear in $W$. Since the edge $e_{0}$ joins $a_{0}$ to $a_{1}$, we know that both $a_{0}$ and $a_{1}$ appear in block $B_{0}$. Consequently, $P^{\prime}=C_{1}, \ldots, C_{t}, B_{0}, C_{t+1}, \ldots, C_{t+r}, B_{1}$ is a trail in $G_{S}$. Continuing in this manner produces a Hamilton cycle in $G_{S}$ due to the fact that together the elements of $W$ touch every block of $\mathcal{B}$, so every block will eventually be included in the path.

The terminology used in [2] is somewhat confusing. We take this opportunity to introduce some new terminology which will also be required in generalizing this method to proving the existence of rank two Ucycles. Let $S=(V, \mathcal{B})$ be a $(v, K, 1)$ PBD. Let $P$ be a trail in $G^{V}$ with every edge a different colour. Suppose there are $\ell$ points in $P$ and let $n$ denote the number of distinct points in $P$. Clearly $n \leq \ell$. Suppose that $P$ is not block-dominating and let $B=\left\{x_{0}, x_{1}, \ldots, x_{k-1}\right\} \in \mathcal{B}$ be a block that does not intersect $P$. Let $Q_{B}$ be the set of edges in $P$ (which by definition have neither endpoint in $B$ ) representing blocks that intersect $B$, i.e. $Q_{B}=\left\{e \mid e \in E(P), B_{e} \cap B \neq \emptyset\right\}$. The edge $e=\left\{v_{i}, v_{i+1}\right\}$ in Figure 32 represents a $Q_{B}$-type edge.

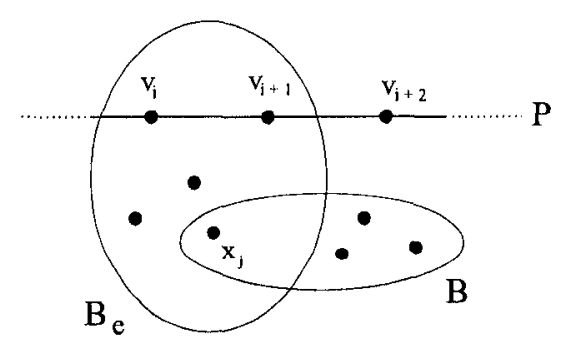

Figure 32: Edge $e=\left\{v_{i}, v_{i+1}\right\}$ is $Q_{B}$-type, where $B$ is a block not intersecting $P$ 
We now prove Theorem 6.1.1 using the method presented in [2] but employing the terminology introduced above.

Proof of Theorem 6.1.1. Assume $S=(V, \mathcal{B})$ is a non-trivial $(v, K, 1)$-PBD satisfying the block size restrictions (i.e. $M \leq 2 m$ ). We construct a block-dominating closed trail in $G^{V}$ which, appealing to Lemma 6.1 .2 , implies the existence of a Hamilton cycle in the block intersection graph of $S$.

Since $S$ is non-trivial there are three points, say $x, y, z \in V$, not all in the same block, hence $P=x, y, z$ is a 3-cycle in $G^{V}$ with all three edges of different colour. If $P$ is block-dominating we are done. Otherwise, we must extend $P$ to a longer cycle with edges of different colours. We claim that we eventually obtain a block-dominating cycle.

Suppose $P$ is a cycle in $G^{V}$ all of whose edges have different colours, and assume that $P=v_{0}, v_{1}, \ldots, v_{\ell-1}$. Let $B=\left\{x_{0}, x_{1}, \ldots, x_{k-1}\right\} \in \mathcal{B}$ be a block that does not intersect $P$, that is, $B \cap\left\{v_{0}, v_{1}, \ldots, v_{\ell-1}\right\}=\emptyset$. We give two methods for extending $P$ to block-dominate $B$. Suppose there are two consecutive $Q_{B}$-type edges in $P$, $e_{i-1}=\left\{v_{i-1}, v_{i}\right\}$ and $e_{i}=\left\{v_{i}, v_{i+1}\right\}$. Without loss of generality, let $x_{s} \in B_{e_{i-1}}$ and $x_{t} \in B_{e_{i}}, s, t \in\{0,1, \ldots, k-1\}$, where $x_{s} \neq x_{t}$. We must have $x_{s} \neq x_{t}$ since if $x_{s}$ were equal to $x_{t}$, the points $v_{i}$ and $x_{s}$ would appear in both $B_{e_{i-1}}$ and $B_{e_{i}}$, implying $B_{e_{i-1}}=B_{e_{i}}$ because $\lambda=1$. This would imply that $P$ has two edges of the same colour which is not true. $P$ can be extended by replacing the path $v_{i-1}, v_{i}, v_{i+1}$ with 
the path $v_{i-1}, x_{s}, x_{t}, v_{i+1}$. This replacement does not remove any edge colours from $P$ and adds one new edge colour. Suppose there are no consecutive $Q_{B}$-type edges in $P$, but that the edge $e_{i}=\left\{v_{i}, v_{i+1}\right\}$ in $P$ is a $Q_{B}$-type edge such that $B_{e_{i}}$ intersects $B$ in $x_{s}$, where $s \in\{0,1, \ldots, k-1\}$. If there is an edge $\left\{v_{i}, x_{t}\right\}$ or an edge $\left\{v_{i+1}, x_{t}\right\}$, $t \in\{0,1, \ldots, k-1\}$, where $t \neq s$, whose colour does not appear in $P$, then $P$ can be extended to a longer cycle by replacing the path $v_{i}, v_{i+1}$ with the path $v_{i}, x_{t}, x_{s}, v_{i+1}$ or the path $v_{i}, x_{s}, x_{t}, v_{i+1}$. This insertion adds two new colours to $P$ and removes none.

The proof rests on the claim that $P$ can be extended using the methods explained above until a block-dominating cycle is obtained. Suppose that $P$ cannot be extended and let $B$ be a block that does not intersect $P$. We can assume that the $Q_{B}$-type edges in $P$ form a matching, since if any two $Q_{B}$-type edges are adjacent in $P, P$ can be extended. We can also assume that if $\left\{v_{i}, v_{i+1}\right\}$ is a $Q_{B}$-type edge in $P$, then the colours of the edges $\left\{v_{i}, x_{t}\right\}$ and $\left\{v_{i+1}, x_{t}\right\}$ already appear in $P$ for every $x_{t} \in B$. Let $q$ be the number of $Q_{B}$-type edges in $P$. It is necessary to deal with two cases: $q=0$ and $q \neq 0$. The proof presented by Alspach, Heinrich and Mohar in [2] neglects the possibility that $q=0$, however, it is possible that $q=0$ and we deal with this case first. Since $P$ does not dominate the block $B=\left\{x_{0}, x_{1}, \ldots, x_{k-1}\right\}$, no $x_{j}$, for $0 \leq j \leq k-1$, appears in $P$. Also, since there are no $Q_{B}$-type edges in $P$, none of the blocks induced by the edges of $P$ intersect $B$. Each point in $P$ appears together 
with each $x_{j}, 0 \leq j \leq k-1$, in some block because $\lambda=1$, therefore, we can extend $P$ by replacing the edge $\left\{v_{0}, v_{1}\right\}$ with the path $\left\{v_{0}, x_{0}\right\},\left\{x_{0}, x_{1}\right\},\left\{x_{1}, v_{1}\right\}$. Each of these edges has a distinct colour and none of these colours appears in $P$. This contradicts the assumption that $P$ cannot be extended.

Now suppose $q \geq 1$. Let the sizes of the blocks corresponding to $Q_{B}$-type edges be denoted $k_{0}, \ldots, k_{q-1}$ and let the blocks represented by $Q_{B}$-type edges be denoted $B_{q, i}, i \in\{0,1, \ldots, q-1\}$. Since no two edges in $P$ can be the same colour, no other edge with a colour the same as a $Q_{B}$-type edge can be added to $P$. Such edges exist between the vertices of $P$ and elements of $B$. We want to know how many edges of colour $B_{q, i}$ go between $V(P)$ and $B$. Only one point of $B_{q, i}$ can be in $B$, otherwise a pair of points would appear in two different blocks. How many points of $B_{q, i}$ can be in $P$ ? All of them except the point in which $B_{q, i}$ and $B$ intersect. There are $\left|B_{q, i}\right|=k_{i}$ points in $B_{q, i}$ so there are at most $k_{i}-1$ of these in $P$. The largest number of edges that cannot be considered for inclusion in $P$ is

$$
\left(k_{0}-1\right)+\ldots+\left(k_{q-1}-1\right)=\sum_{i=0}^{q-1} k_{i}-q .
$$

This means there are at least

$$
k \ell-\left(\sum_{i=0}^{q-1} k_{i}-q\right)=k \ell+q-\sum_{i=0}^{q-1} k_{i}
$$

edges between $V(P)$ and $B$ whose colours do not appear in $P$, where $k \ell$ is the total number of edges between vertices of $P$ and elements of $B$. Since there are $q Q_{B}$-type 
edges in $P$, the $Q_{B}$-type edges have $2 q$ distinct endpoints (remember that no two $Q_{B}$-type edges are incident), and therefore, all edges between $V(P)$ and $B$ whose colours do not appear in $P$ appear at the remaining $\ell-2 q$ vertices. The number of these edges is $k(\ell-2 q)$, hence,

$$
(\ell-2 q) k \geq u \geq k \ell+q-\sum_{i=0}^{q-1} k_{i}
$$

where $u$ is the number of edges between $V(P)$ and $B$ whose colours do not already appear in $P$. Finally,

$$
k \ell+q-\sum_{i=0}^{q-1} k_{i} \geq k \ell+q-q M,
$$

so $(\ell-2 q) k-(k \ell+q-q M) \geq 0$ which yields $q M-q-2 q k \geq 0$. Therefore, we have $M-1-2 k \geq 0$, which implies $M \geq 2 k+1$. This is a contradiction to the condition that $M \leq 2 m$, since the minimum value of $k$ is $m$. We conclude that it is always possible to extend $P$ when $q \geq 1$.

The existence of a Hamilton cycle in the block intersection graph of a $(v, K, 1)$ PBD does not imply the existence of a rank two Ucycle for the blocks of the PBD. For example, if the Hamilton cycle contains the sub-path $\{0,1,3\},\{0,4,5\},\{0,2,6\}$ it is impossible to translate this into a Ucycle of rank two as all three blocks share the element 0 . To determine a Ucycle of rank two for a design $S=\{V, \mathcal{B}\}$, we must find a cycle $P$ in $G^{V}$ which is edge block-dominating and which has no two edges the same colour. A trail is called edge block-dominating if the set of edges in the 
trail intersect every block of the design. The following lemma is the rank two Ucycle companion to Lemma 6.1.2 for $(v, K, 1)$-PBDs.

Lemma 6.1.3. Let $S=(V, \mathcal{B})$ be $a(v, K, 1)-P B D . S$ admits a Ucycle of rank two if and only if $G^{V}$ has an edge block-dominating closed trail $W$ with no two edges of $W$ having the same colour.

Proof. Let $S=(V, \mathcal{B})$ be a $(v, K, 1)$-PBD. $G^{V}$ is the complete graph on $|V|$ vertices. An edge block-dominating closed trail in $G^{V}$ with no two edges having the same colour must contain exactly $|\mathcal{B}|$ edges, since each edge represents a unique block. Suppose the edge block-dominating trail is $v_{0}, e_{0}, v_{1}, e_{1}, \ldots, e_{b-1}, v_{b-1}$, where each $e_{i}$, $i \in\{0,1, \ldots, b-1\}$, is a unique edge in $G^{V}$, but the points $v_{i}, i \in\{0,1, \ldots, b-1\}$, are not necessarily unique. The sequence $U=v_{0}, v_{1}, \ldots, v_{b-1}$ is a rank two Ucycle for $S$. The reverse argument holds since each pair of points in a rank two Ucycle represents a unique block of $S$, and therefore a unique edge in $G^{V}$, and every block of $S$ is represented exactly once.

Let $S=(V, \mathcal{B})$ be a $(v, K, 1)$-PBD with $M \leq 2 m$. Alspach, Heinrich and Mohar's result implies that given $G^{V}$, we can always find a trail $P$, with no two edges of the same colour, that is block-dominating. How do we make this trail edge blockdominating?

Suppose we have a block-dominating trail $P$ with no two edges of the same colour. Each edge in $P$ represents a unique block and every block has at least one of its points 
in $P$. Suppose that the block $B=\left\{x_{0}, x_{1}, \ldots, x_{k-1}\right\}$ is not represented by an edge in $P$. We adjust the definition of $Q_{B}$ as follows: $Q_{B}=\left\{e \mid e \in E(P),\left(B_{e} \backslash e\right) \cap B \neq\right.$ $\emptyset$. Let $R_{B}$ denote the set of edges in $P$ with one endpoint intersecting $B$, that is, $R_{B}=\{e|e \in E(P)|, e \cap B \mid=1\}$. Let $S_{B}$ be the set of edges in $P$ whose associated blocks do not intersect $B$, i.e. $S_{B}=\left\{e \mid e \in E(P), B_{e} \cap B=\emptyset\right\}$. Let $T_{B}$ be the set of blocks, other than $B$, that are not represented by an edge in $P$ and that intersect $B$. Finally, let $E_{B}$ represent the set of edges that can be used to represent $B$, that is, pairs of points from $B$.

In the proof of Theorem 6.1.1, the $Q_{B}$-type edges were used to extend a trail to a block-dominating trail. What methods can we use to extend a block-dominating trail to an edge block-dominating trail? Let $P=v_{0}, e_{0}, v_{1}, e_{1}, \ldots, v_{m}$ and let $B=$ $\left\{x_{0}, x_{1}, \ldots, x_{k-1}\right\}$ be a block not edge block-dominated by $P$.

Extension 6.1.1. If $e_{i}=\left\{v_{i}, v_{i+1}\right\}$ and $e_{i+1}=\left\{v_{i+1}, v_{i+2}\right\}$ are $Q_{B}$-type edges, with $x_{s} \in B_{e_{i}}$ and $x_{t} \in B_{e_{i+1}}, s, t \in\{0,1, \ldots, k-1\}$, where $s \neq t$, then $P$ can be extended to edge block-dominate $B$ by replacing $v_{i}, v_{i+1}, v_{i+2}$ with $v_{i}, x_{s}, x_{t}, v_{i+2}$.

Extension 6.1.1 is represented in Figure 33, with green edges indicating the block $B_{e_{i}}$ and red edges indicating the block $B_{e_{i+1}}$.

Extension 6.1.2. If $e_{i}=\left\{v_{i}, v_{i+1}\right\}$ is a $Q_{B}$-type edge, with $x_{s} \in B_{e_{i}}, s \in\{0,1, \ldots, k-$ $1\}$, and if edge $\left\{v_{i+1}, x_{t}\right\}$ (or edge $\left.\left\{v_{i}, x_{t}\right\}\right), t \in\{0,1, \ldots, k-1\}$, represents a $T_{B^{-}}$ type block, then $P$ can be extended to edge block-dominate $B$ by replacing $v_{i}, v_{i+1}$ with 


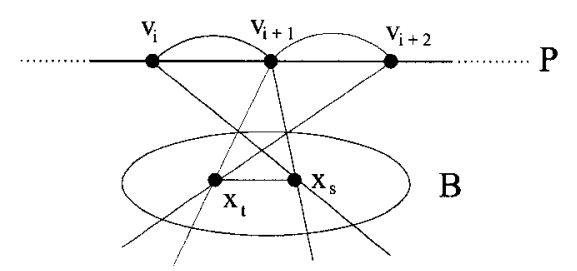

Figure 33: Extension 6.1.1

$v_{i}, x_{s}, x_{t}, v_{i+1}\left(\right.$ or $\left.v_{i}, x_{t}, x_{s}, v_{i+1}\right)$

Extension 6.1.2 is represented in Figure 34, with green edges indicating the block $B_{e_{i}}$ and $B_{t}$ being the $T_{B}$-type block (with red edge indicating the edge that makes $B_{t}$ a $T_{B}$-type block).

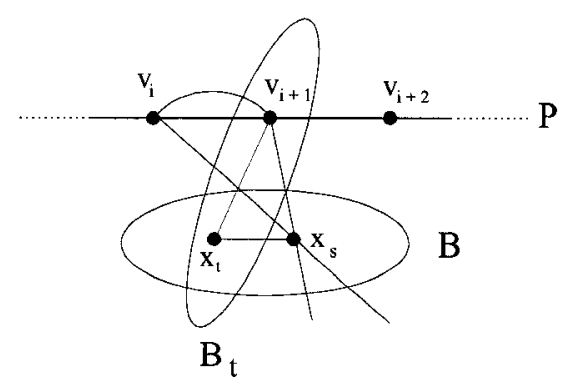

Figure 34: Extension 6.1.2

Extension 6.1.3. If $\left\{v_{i}, x_{s}\right\}, s \in\{0,1, \ldots, k-1\}$, is a $R_{B}$-type edge (note that $x_{s}$ must also be in $P$ ) and if edge $\left\{x_{t}, v_{i}\right\}, t \in\{0,1, \ldots, k-1\}$, represents a $T_{B}$-type block, then $P$ can be changed by replacing $v_{i}, x_{s}$ with $v_{i}, x_{t}, x_{s}$.

While extension 6.1 .3 will result in the loss of one block from the set of blocks edge block-dominated by $P$, it does increase the length of $P$ by one by adding two new edges to $P$. Extension 6.1.3 is represented in Figure 35, with the green edge indicating 
the $R_{B}$-type edge and $B_{t}$ being the $T_{B}$-type block (with red edge indicating the edge that makes $B_{t}$ a $T_{B}$-type block).

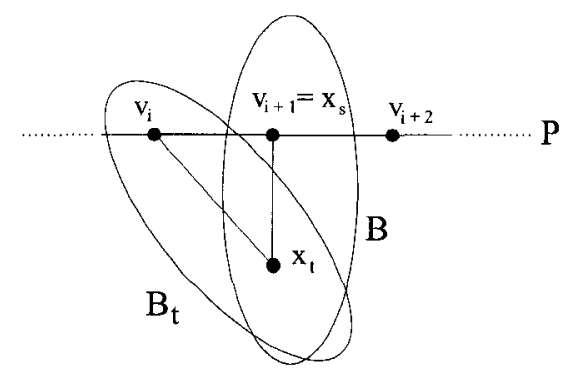

Figure 35: Extension 6.1.3

Extension 6.1.4. If $\left\{v_{i}, v_{i+1}\right\}$ is a $S_{B}$-type edge and if two edges, $\left\{x_{s}, v_{i}\right\}$ and $\left\{x_{t}, v_{i+1}\right\}, s, t \in\{0,1, \ldots, k-1\}$, incident to this edge represent $T_{B}$-type blocks, then $P$ can be extended by replacing $v_{i}, v_{i+1}$ with $v_{i}, x_{s}, x_{t}, v_{i+1}$ or $v_{i}, x_{s}, v_{i+1}\left(\right.$ if $x_{t}=x_{s}$ ).

Extension 6.1.4 is represented in Figure 36, the coloured edges are both representatives of $T_{B}$-type blocks. Note that it is possible that $x_{t}=x_{s}$.

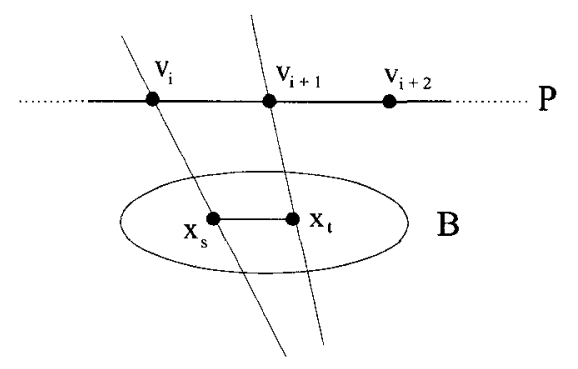

Figure 36: Extension 6.1.4

Extension 6.1.5. If $v_{i}$ is incident to two edges, $\left\{x_{s}, v_{i}\right\}$ and $\left\{x_{t}, v_{i}\right\}, s, t \in\{0,1, \ldots, k-$ $1\}$, where $s \neq t$, each representing a distinct $T_{B}$-type block, then $P$ can be extended 
by replacing $v_{i}$ with $v_{i}, x_{s}, x_{t}, v_{i}$.

Extension 6.1 .5 is represented in Figure 37, the coloured edges are both representatives of $T_{B}$-type blocks. Note that $x_{s} \neq x_{t}$ otherwise the pair of points $\left\{v_{i}, x_{s}\right\}$ would appear in two distinct blocks.

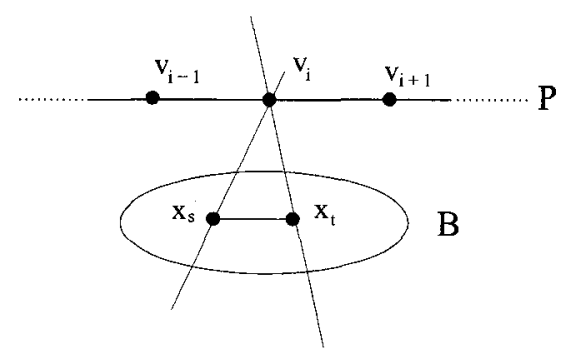

Figure 37: Extension 6.1.5

In order to prove the existence of a rank two Ucycle for a $(v, K, 1)$-PBD, $S=$ $(V, \mathcal{B})$, we must show that an edge block-dominating cycle with no two edges the same colour exists in $G^{V}$. Adopting the method of Alspach, Heinrich and Mohar, we assume that we can find a trail $P$ in $G^{V}$ that is block-dominating but is not edge blockdominating and that there is no way to extend $P$. Extension 6.1 .1 implies that no pair of $Q_{B}$-type edges are adjacent if $P$ cannot be extended. Extensions 6.1 .2 and 6.1 .3

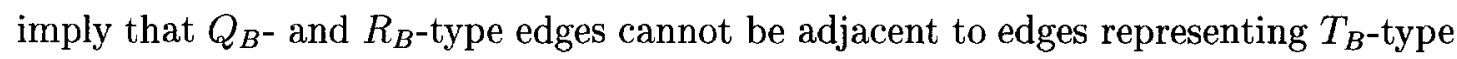
blocks if $P$ cannot be extended. Extension 6.1.4 implies that at most one edge incident to an $S_{B}$-type edge can represent a $T_{B}$-type block if $P$ cannot be extended. Finally, Extension 6.1.5 implies that each vertex in $P$ has at most one edge representing a $T_{B}$-type block incident if $P$ cannot be extended. By counting the number of edges 
of each type that must occur in $P$ when $P$ cannot be extended, we hope to reach a contradiction. The difficulty lies in counting the number of edges of each type in $G^{V}$ for a given block $B$.

Although we cannot yet say whether Ucycles of rank two exist in general for $(v, K, 1)$-PBDs, we do have the following non-existence result (first reported as The-

orem 4.2.1): Given $v \in \mathbb{N}$ and any partition of $v=\sum_{i=1}^{\ell} v_{i}$ such that $v_{1} \geq 3$ and $v_{i} \geq 2$, for all $i \in\{2,3, \ldots, \ell\}$, there exists a $\left(v, 2 \cup\left\{v_{i} \mid 1 \leq i \leq \ell\right\}, 1\right)$-PBD that does not admit a rank two Ucycle. We also know that the $(6,\{2,3\}, 1)$-PBD created by deleting a point from the Fano plane does not admit a Ucycle of rank two.

\subsection{Conjectures and Questions}

Here we gather the conjectures stated throughout this dissertation and present some questions stemming from our work.

\subsubsection{Conjectures}

In Section 4.2 we conjectured the following.

Conjecture 6.2.1. Every $(v, K, \lambda)-P B D$, with $\min K \geq 3$ and a sufficient number of non-regular base blocks, admits a Ucycle of rank two.

While we believe this conjecture is true, it is likely that the first steps in the 
search for Ucycles of rank two for general (not necessarily cyclic) PBDs will begin with proving the following two conjectures.

Conjecture 6.2.2. Every $(v, k, 1)-B I B D$, with $k \geq 3$, admits a Ucycle of rank two.

Conjecture 6.2.3. Every $(v, k, \lambda)-B I B D$, with $k \geq 3$, admits a Ucycle of rank two.

These conjectures and our examples of designs that do not admit Ucycles of rank two indicate that blocks of size two are problematic and must be investigated further.

Our results regarding the existence of Ucycles of rank three for TTS $(v) \mathrm{s}$, with $v \equiv 1,4,7(\bmod 12)$, can likely be extended to $v \equiv 10(\bmod 12)$. Recall that we have shown by computer search that no TTS(10) admits a rank three Ucycle.

Conjecture 6.2.4. For each $v \equiv 10(\bmod 12), v \geq 22$, there exists a TTS $(v)$ that admits a Ucycle of rank three.

We also believe it is possible to "fill in the gaps" in Theorem 4.1.4.

Conjecture 6.2.5. (i) For each $v \equiv 1(\bmod 12)$, with $v \equiv 0(\bmod 5)$, there exists a TTS $(v)$ that admits a Ucycle of rank three.

(ii) For each $v \equiv 4(\bmod 12)$, with $v \equiv 0(\bmod 5)$, there exists a TTS $(v)$ that admits a Ucycle of rank three.

We have shown that Ucycles of rank three cannot exist for TTS $(v)$ s with $v \equiv$ $0,3,6,9(\bmod 12)$, but for each $v \equiv 3(\bmod 12), v>3$, there exists a $\operatorname{TTS}(v)$ admitting a 2-intersecting Gray cycle. Techniques similar to those used in proving this 
result should be applicable to $\operatorname{TTS}(v)$ s with $v \equiv 0,6,9(\bmod 12)$.

Conjecture 6.2.6. For each $v \equiv 0,6,9(\bmod 12)$, there exists a TTS $(v)$ that admits a -intersecting Gray code.

As discussed in Section 4.1, M. Colbourn and Johnstone's work on the nonexistence of 2-intersecting Gray codes for $\operatorname{TTS}(v) \mathbf{s}$ is incomplete. They describe a construction method for determining twofold triple systems that do not admit minimal change orderings for their blocks, however, they provide only one example of the construction in action. Because the construction involves the assumption that a graph with special properties can be completed to form the 2-block intersection graph of some $\operatorname{TTS}(v)$, it is not clear that the construction can be applied to create a family of graphs having this property. Recall that 2-intersecting Gray codes are equivalent to $A_{3}$-orderings.

Conjecture 6.2.7. There exists $v_{0} \in \mathbb{Z}^{+}$such that, for all $v \geq v_{0}$, there exists at least one TTS $(v)$ that does not admit a 2-intersecting Gray code.

\subsubsection{Questions}

In this section, we list some questions stemming from our research. Some of these questions are directly related to this research and will be pursued further, while others are more tangential. 
Question 6.2.1. Robert Jamieson asked if rank two Ucycles for cyclic $(v, k, \lambda)$-BIBDs - which we create by construction - can be balanced.

More specifically, Question 6.2.1 asks, in a rank two Ucycle for a $(v, k, \lambda)$-BIBD, $S=(V, \mathcal{B})$, does each $v \in V$ appear an equal (or as close as possible) number of times? This is analogous to the question of existence of balanced Gray codes, however, in the case of Gray codes, we look at the transition sequence of the Gray code, not at the contents of the Gray code itself (which is certainly balanced). We believe the answer to Question 6.2.1 is likely yes. Our construction method for partial Ucycles of rank two ensures balance initially. The insertion of short orbit blocks may disrupt this balance, however, we hypothesize that it is possible to maintain balance through careful selection of insertion points.

Theorem 4.2.1 identifies an infinite family of PBDs that do not admit Ucycles of rank two. In addition, there exists a $(6,\{2,3\}, 1)$-PBD that does not admit a Ucycle of rank two.

Question 6.2.2. Are there other families of designs that do not admit rank two Ucycles?

Question 6.2.3. Are there other natural ways to define Ucycles for block designs? In particular, are there other choices for rank besides block size and strength that yield interesting and general results? 
In constructing rank three Ucycles and 2-intersecting Gray cycles for TTS $(v)$ s we developed the concept of a difference sequence.

Question 6.2.4. Do difference sequences (cyclic or non-cyclic) exist for all cyclic $T T S(v) s ?$

Configuration orderings for $(v, k, \lambda)$-BIBDs have not been well-studied apart from triple systems. As noted in Chapter 5, the existence of standard configuration orderings where the configurations contain a small number of blocks are not all determined.

Question 6.2.5. Do $T S(v, \lambda) s$ admit $B_{4}$-orderings? Do $T S(v, \lambda) s$ admit $B_{5}$-orderings?

Question 6.2.6. Can the known results in standard configuration ordering for triple systems be extended to $(v, k, \lambda)-B I B D s$ ?

In Section 2.3, we noted that for standard binary reflected Gray codes there exist formulas for ranking and unranking. That is, it is possible to determine where a given element appears in the Gray code and, given a position, it is possible to determine which element appears in that position in the list. Ranking and unranking is also possible for many combinatorial Gray codes; however, it is not immediately obvious how to perform ranking and unranking for a $\kappa$-intersecting Gray code for a design. This is due, in part, to the fact that our method of constructing 2-intersecting Gray cycles for cyclic $\operatorname{TTS}(v) \mathbf{s}$ is not recursive. 
Question 6.2.7. Do there exist ranking and unranking functions for 2-intersecting Gray cycles for cyclic triple systems?

As ordering the blocks of designs is an emerging field of study, many interesting and potentially fruitful areas of investigation remain open. 


\section{Bibliography}

[1] B. Alspach and D. Hare. Edge-pancyclic block-intersection graphs. Discrete Math., 97:17-24, 1991.

[2] B. Alspach, K. Heinrich, and B. Mohar. A note on Hamilton cycles in blockintersection graphs. Contemp. Math., 111:1-4, 1990.

[3] S. Batzoglou and S. Istrail. Physical mapping with repeated probes: the hypergraph superstring problem. J. Discrete Algorithms, 1(1):51-76, 2000.

[4] G. S. Bhat and C. D. Savage. Balanced Gray codes. Electron. J. Combin., 3(1), 1996.

[5] J. R. Bitner, G. Ehrlich, and E. M. Reingold. Efficient generation of the binary reflected gray code and its applications. Comm. ACM, 19(9):517-521, 1976.

[6] R. A. Brualdi. Introductory Combinatorics. Prentice-Hall, Upper Saddle River, NJ, 1999. 
[7] M. Buck and D. Wiedemann. Gray codes with restricted density. Discrete Math., 48:163-171, 1984.

[8] J. Buhler and B. Jackson. Private communication, 2006.

[9] G. A. Case and D. A. Pike. Pancyclic PBD block-intersection graphs. Discrete Math. To appear.

[10] K. Casteels. Universal cycles for $(n-1)$-partitions of an $n$-set. Master's thesis, Carleton University, Ottawa, ON, 2004.

[11] P. J. Chase. Combination generation and graylex ordering. Congr. Numer., $69: 215-242,1989$.

[12] F. Chung, P. Diaconis, and R. Graham. Universal cycles for combinatorial structures. Discrete Math., 110:43-59, 1992.

[13] M. B. Cohen and C. J. Colbourn. Steiner triple systems as multiple erasure correcting codes in disk arrays. In Proceedings of IPCCC 2000 (19th IEEE International Conference on Performance, Computing and Communications), pages 288-294, 2000.

[14] M. B. Cohen and C. J. Colbourn. Ordering disks for double erasure codes. In Proc. Symp. Parallel Algorithms and Architectures (SPAA01), pages 229-236, 2001. 
[15] M. B. Cohen and C. J. Colbourn. Optimal and pessimal orderings of Steiner triple systems in disk arrays. Theoret. Comput. Sci., 297:103-117, 2003.

[16] M. B. Cohen and C. J. Colbourn. Ladder orderings of pairs and RAID performance. Disc. Appl. Math., 138(1):35-46, 2004.

[17] M. B. Cohen, C. J. Colbourn, and D. Froncek. Cluttered orderings for the complete graph. In Lecture Notes in Comput. Sci., volume 2108, pages 420-431, 2001.

[18] C. J. Colbourn, M. J. Colbourn, J. J. Harms, and A. Rosa. A complete census of $(10,3,2)$ block designs and of Mendelsohn triple systems of order 10. III. $(10,3,2)$ block designs without repeated blocks. Congr. Numer., 37:211-234, 1983.

[19] C. J. Colbourn and A. Rosa. Triple systems. Oxford Mathematical Monographs. The Clarendon Press Oxford University Press, New York, 1999.

[20] M. J. Colbourn and J. K. Johnstone. Twofold triple systems with a minimal change property. Ars Combin., 18:151-160, 1984.

[21] M. J. Colbourn and R. A. Mathon. On cyclic Steiner 2-designs. Ann. Disc. Math., 7:215-253, 1980.

[22] M. Cooke, M. Dewar, C. North, and B. Stevens. Beckett Gray codes. Submitted to Discrete Mathematics, 2007. 
[23] P. Eades, M. Hickey, and R. C. Read. Some Hamilton paths and a minimal change algorithm. J. Assoc. Comput. Mach., 31(1):19-29, 1984.

[24] P. Eades and B. McKay. An algorithm for generating subsets of fixed size with a strong minimal change property. Inform. Process. Lett., 19(3):131-133, 1984.

[25] H. Fredricksen. A survey of full length nonlinear shift register cycle algorithms. SIAM Rev., 24(2):195-221, 1982.

[26] M. J. Grannell and T. S. Griggs. Configurations in Steiner triple systems. In Combinatorial designs and their applications (Milton Keynes, 1997), volume 403 of Chapman \& Hall/CRC Res. Notes Math., pages 103-126. 1999.

[27] M. J. Grannell, T. S. Griggs, and E. Mendelsohn. A small basis for four-line configurations in Steiner triple systems. J. Combin. Des, 3(1):51-49, 1995.

[28] M. J. Grannell, T. S. Griggs, and C. A. Whitehead. The resolution of the antiPasch conjecture. J. Combin. Des., 8(4):300-309, 2000.

[29] F. Harary, R. W. Robinson, and N. C. Wormald. Isomorphic factorisations I: Complete graphs. Trans. Amer. Math. Soc., 242:243-260, 1978.

[30] D. R. Hare. Cycles in the block-intersection graph of pairwise balanced designs. Discrete Math., 137:211-221, 1995. 
[31] L. Hellerstein, G. A. Gibson, R. M. Karp, R. H. Katz, and D. A. Patterson. Coding techniques for handling failures in large disk arrays. Algorithmica, 12:182-208, 1994.

[32] P. Horák, D. A. Pike, and M. E. Raines. Hamilton cycles in block-intersection graphs of triple systems. J. Combin. Des., 7(4):243-246, 1999.

[33] P. Horák and A. Rosa. Decomposing Steiner triple systems into small configurations. Ars Combin., 26:91-105, 1988.

[34] P. Horák and A. Rosa. Private communication, 2005.

[35] G. Hurlbert. On universal cycles for $k$-subsets of an $n$-set. SIAM J. Disc. Math., $7(4): 598-604,1994$.

[36] G. Hurlbert. Multicover ucycles. Discrete Math., 137:241-249, 1995.

[37] G. Hurlbert and G. Isaak. Equivalence class universal cycles for permutations. Discrete Math., 149:123-129, 1996.

[38] G. H. Hurlbert. Universal Cycles: On beyond De Bruijn. PhD thesis, Rutgers University, New Brunswick, NJ, 1990.

[39] B. W. Jackson. Universal cycles of $k$-subsets and $k$-permutations. Discrete Math., $117: 141-150,1993$. 
[40] D. E. Knuth. The Art of Computer Programming, Volume 4,. Addison-Wesley, Upper Saddle River, NJ, 2005.

[41] V. L. Kompel'maker and V. A. Liskovets. Successive generation of permutations by means of a transposition basis. Cybernetics, 11(3):362-366, 1976.

[42] D. L. Kreher and D. R. Stinson. Combinatorial Algorithms: Generation, Enumeration and Search. CRC Press, Boca Raton, FL, 1999.

[43] M. H. Martin. A problem in arrangements. Bull. Amer. Math. Soc., 40:859-864, 1934.

[44] B. McKay. Private communication, 2006.

[45] F. Ruskey. Adjacent interchange generation of combinations. J. Algorithms, $9(2): 162-180,1988$

[46] F. Ruskey. Simple combinatorial Gray codes constructed by reversing sublists. In Lecture Notes in Comput. Sci., volume 762, pages 201-208, 1993.

[47] C. Savage. A survey of combinatorial Gray codes. SIAM Rev., 39(4):605-629, 1997.

[48] C. D. Savage and P. Winkler. Monotone Gray codes and the middle levels problem. J. Combin. Theory. Ser. A, 70(2):230-248, 1995.

[49] G. J. Simmons and J. A. Davis. Pair designs. Comm. Statist., 4:255-272, 1975. 
[50] P. J. Slater. Generating all permutations by graphical transpositions. Ars Combin., 5:219-225, 1978.

[51] N. J. A. Sloane. The On-Line Encyclopedia of Integer Sequences. Published electronically, www.research.att.com/ ^njas/sequences/, 2004.

[52] N. J. A. Sloane. Table of $n, \operatorname{phi}(n)$ for $n=1 \ldots$ 10000. Published electronically, www.research.att.com/ njas/sequences/b000010.txt, 2007.

[53] B. Stevens, P. Buskell, P. Ecimovic, C. Ivanescu, A. Malik, A. Savu, T. Vassilev, H. Verrall, B. Yang, and Z. Zhao. Solution of an outstanding conjecture: the non-existence of universal cycles with $k=n-2$. Discrete Math., 258:193-204, 2002.

[54] D. R. Stinson. Combinatorial Designs: Constructions and Analysis. Springer, New York, 2004.

[55] V. E. Vickers and J. Silverman. A technique for generating specialized Gray codes. IEEE Trans. Comput., 29(4):329-331, 1980.

[56] D. G. Wagner and J. West. Construction of uniform Gray codes. Congr. Numer., $80: 217-223,1991$.

[57] W. D. Wallis. Combinatorial Designs. Marcel Dekker, Inc., New York, 1988. 
[58] D. B. West. Introduction to Graph Theory, Second Edition. Prentice Hall, Upper Saddle River, NJ, 2001.

[59] H. S. Wilf. Combinatorial algorithms: an update. CBMS-NSF Regional Conference Series in Applied Mathematics, 55. Society for Industrial and Applied Mathematics (SIAM), Philadelphia, PA, 1989. 


\section{Index}

$(p, \ell)$-configuration, 24

$(v, K, \lambda)-\mathrm{PBD}, 23$

$(v, k, \lambda)$-BIBD, 13

$\kappa$-intersecting Gray code, 62

$n$-line configuration, 24

$t-(v, k, \lambda)$ design, 13

automorphism, 17

base block, 17

full orbit, 19

non-regular, 123

problem, 193

regular short orbit, 19

short orbit, 19

base block coloured pair adjacency graph,

24

BIBD, 13 1-rotational, 22

$k$-rotational, 22

cyclic, 17

symmetric, 15

binary Gray code, 28

balanced, 31

monotone, 32

standard binary reflected, 28

totally balanced, 31

block coloured pair adjacency graph, 24

block intersection graph, 23

characteristic vector, 28

combinatorial Gray code, 33

configuration, 24

configuration ordering, 74

generalized, 79 
optimal, 76

pessimal, 74

de Bruijn digraph, 39

de Bruijn sequence, 39

design, 12

difference partition, 18

difference sequence, 88

cyclic, 87

difference set, 16

supplementary, 16

edge-pancyclic, 78

erasure-correcting code, 70

Euler cycle, 39

Eulerian design, 109

Hamilton cycle, 50

isomorphism, 17

line graph, 77

minimal change, 33

$k$-subsets of an $n$-set, 33 adjacent, 36

permutations, 37

strong, 35

orbit, 17

full, 18

half, 18

short, 18

pair design, 77

point-block incidence graph, 117

revolving door algorithm, 34

strength, 13

strongly connected, 39

$\operatorname{STS}(v), 13$

transition count, 30

transition digraph, 44

$k$-permutations of $[n], 44$

$k$-subsets of $[n], 53$

partitions of $[n], 58$

permutations of $[n], 49$ 
transition sequence, 29

translate, 16

triple system, 13

Steiner, 13

twofold, 13

$\operatorname{TS}(v, \lambda), 13$

$\operatorname{TTS}(v), 13$

Ucycle for block designs

rank $\kappa, 65$

rank three, 67

rank two, 67

Universal cycle, 43

$t$-cover, 56 